Portland State University

PDXScholar

4-7-2020

\title{
Aggregated Water Heater System (AWHS) Optimization for Ancillary Services
}

Manasseh Obi

Portland State University

Follow this and additional works at: https://pdxscholar.library.pdx.edu/open_access_etds

Part of the Electrical and Computer Engineering Commons Let us know how access to this document benefits you.

\section{Recommended Citation}

Obi, Manasseh, "Aggregated Water Heater System (AWHS) Optimization for Ancillary Services" (2020). Dissertations and Theses. Paper 5428.

https://doi.org/10.15760/etd.7301

This Dissertation is brought to you for free and open access. It has been accepted for inclusion in Dissertations and Theses by an authorized administrator of PDXScholar. Please contact us if we can make this document more accessible: pdxscholar@pdx.edu. 
Aggregated Water Heater System (AWHS) Optimization for Ancillary Services

by

Manasseh Obi

A dissertation submitted in partial fulfillment of the requirements for the degree of

\author{
Doctor of Philosolphy \\ in \\ Electrical and Computer Engineering
}
Dissertation Committee:
Robert Bass, Chair
Dan Hammerstrom
John Acken
Raul Bayoan Cal

Portland State University

2020 
(C) 2020 Manasseh Obi 


Abstract

In this dissertation, I present a two-stage optimization routine that schedules an Aggregated Water Heater System (AWHS) to concurrently provide three utility ancillary services, namely, frequency regulation, frequency response, and peak demand mitigation.

Water heaters can be controlled to manage their energy take, the amount of energy a water heater can absorb upon command. The AWHS is a model aggregation of thousands of water heaters, the energy take and power characteristics of which are based on U.S Census household data and usage behavior patterns. The aggregate energy take available in the AWHS may be dispatched en masse for participation in utility ancillary service markets, while accommodating the unique characteristics of the AWHS resource.

The optimization routine is performed in two stages. In stage one, the optimization routine anticipates future energy take, power, weather temperature, and market prices based on historical data. Upon convergence, the optimization algorithm shifts to the next five minute time interval and re-iterates the optimization with new projected allocations. This process repeats continuously until a full day's worth of projections are simulated. In stage two, the AWHS considers how dispatches affect the AWHS resource, and re-optimizes to maximize revenue for each ancillary service based on forecasted market prices.

The AWHS algorithm relies on forecasts in order to mitigate the effects of overdispatching, which can result in prolonged energy take recovery times. Over-dispatching 
can also lead to lost opportunity cost for the AWHS, and this is prevented by setting dispatch and reserve capacity limits for the ancillary services based on system requirements.

The optimization routine addresses the challenge of dispatching this dynamic resource by assessing how the system recovery can be managed in a way that adequately positions the AWHS to participate in subsequent rounds of bidding. After every dispatch, the available energy take decreases and a new energy curve, the resource recovery curve, is re-calculated. Further, the energy dispatch constraints are dispatch-dependent and need to be recalculated for every selection of dispatch vectors. We propose a solution for calculating the recovery energy take available after a dispatch. This solution slides the entire 24-hour daily window ahead in five minute increments, causing the optimization solution to constantly change as new future data projections are considered.

The primary contribution to knowledge is a solution to the problems caused by Renewable Energy Resources (RER) that uses a novel two-stage method to optimally dispatch the energy take available in the AWHS among the aforementioned three ancillary services in a way that maximizes revenue while minimizing over-dispatching, system recovery time and energy take forecasting errors. 
Dedication

In loving memory of my mother, Maria Catherine Igboahazia Obi 


\section{Acknowledgements}

With special thanks to my advisor and dissertation committee chair, Dr. Robert Bass, who guided me through this doctoral journey. Without his support, technical insights and constant feedback, this PhD would not have been achievable. To the researchers at Portland State University’s Power Lab, Thomas L. Clarke, Emily Barrett, Crystal Eppinger, Jaime Kolln, Rector Blake, and Tylor Slay, thank you for your pursuit of excellence in research. My very special gratitude goes to my research colleague and friend, Kevin Marnell, I consider you a trail blazer.

I would also like to thank my dissertation committee members, Dr. Dan Hammerstrom, Dr. John Acken, and Dr. Raul Bayoan Cal, for all your carefully curated feedback.

My deepest appreciation goes to my wife and children, for allowing me to work on my $\mathrm{PhD}$ while working a full time job, and on the many weeknights and weekends I spent on this research, away from home. I love you all. 


\section{Table of Contents}

Abstract

Dedication

Acknowledgements iv

List of Figures $\quad$ ix

List of Acronyms $\quad$ xi

1 Introduction 1

1.1 Background ........................... 1

1.2 Research Objective . . . . . . . . . . . . . . . . . . . 2

1.3 Research Question . . . . . . . . . . . . . . . . . . . . . . . . 2

1.4 Problem Statement Summary . . . . . . . . . . . . . . . . . 3

1.5 Problem Statement question . . . . . . . . . . . . . . . . 3

1.6 Significance of the proposed solution . . . . . . . . . . . . . 3

1.7 Details of the Problem . . . . . . . . . . . . . . . . . . 4

1.7.1 Stochastic nature of RER . . . . . . . . . . . . . . 4

1.7.2 RER depress overall energy prices . . . . . . . . . . . . 5

1.7.3 Impact on the resiliency and reliability of the grid . . . . . . . . 6

1.7.4 Large scale adoption . . . . . . . . . . . . . . 7

1.7.5 Reduction in power system inertia . . . . . . . . . . . . . . 7

1.7.6 Transmission congestion .................... 8

1.7 .7 Curtailments ....................... 9

1.8 Available Solutions to the Problem and Current Practices . . . . . . . . . . 10

1.8.1 Energy Storage Systems (ESS) . . . . . . . . . . . . . . . . 10

1.8.2 Use of Dedicated Traditional Generation Resources (TGR) for Ancillary Services . . . . . . . . . . . . . . . . . 13

1.8.2.1 Frequency Regulation . . . . . . . . . . . . . . . 14

1.8.2.2 Frequency Response . . . . . . . . . . . . . . . . 15

1.8.2.3 Peak Demand Mitigation . . . . . . . . . . . . . . . . . 19

1.8.2.4 Ramp Rate Control . . . . . . . . . . . . . . . . 22

1.8.2.5 Voltage/Reactive power (VAr) compensation . . . . . . 22

1.8.2.6 Reserves ................. 23 
1.8.3 Dispatchable Standby Generation (DSG) . . . . . . . . . . . . 23

1.8.4 Hybrid Energy Systems (HES) . . . . . . . . . . . . . . . . 25

1.8.5 Demand Side Management (DSM) . . . . . . . . . . . . 26

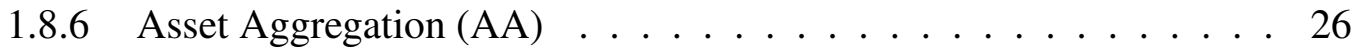

1.9 Primary contribution to knowledge . . . . . . . . . . . . . . . 28

1.10 Motivation . . . . . . . . . . . . . . . . . . . . . . 29

2 Literature Review 31

2.1 Literature Review of select current solutions . . . . . . . . . . . . . 31

2.1.1 Hybrid Energy Systems (HES) . . . . . . . . . . . . . . . 32

2.1.2 Demand Side Management (DSM) . . . . . . . . . . . . . . 34

2.1.3 Asset Aggregation . . . . . . . . . . . . . . . . . . . . . 39

2.1.4 Aggregation of Electric Water Heaters (EWH) . . . . . . . . . . 45

2.1.5 Aggregation of Other Assets . . . . . . . . . . . . . 47

2.2 Literature Survey of Select Optimization Techniques . . . . . . . . . . 49

2.2.1 Particle Swarm Optimization . . . . . . . . . . . . . . . . . 49

2.2.2 Hybrid Big Bang-Big Crunch _ . . . . . . . . . . . . . 51

2.2.3 Time Series Regression Analysis . . . . . . . . . . . . . . . 52

2.2.4 Other Optimization Techniques . . . . . . . . . . . . . . . . . . 52

2.2.5 Related Optimization Work . . . . . . . . . . . . . . . . 53

2.3 Dispatch of Residential Water Heaters for Ancillary Service . . . . . . . . 55

3 Methodology 57

3.1 Introduction . . . . . . . . . . . . . . . 57

3.2 Justification for the proposed methodology used in this research . . . . . 59

3.2.1 Stage 1 and Stage 2 Optimization _. . . . . . . . . . . . 59

3.2 .2 Time shifting . . . . . . . . . . . . . . . 60

3.2.3 Time Series Forecasting . . . . . . . . . . . . . . . . 61

3.2 .4 RNNs and LSTM . . . . . . . . . . . . . . . . . . . . 62

3.2 .5 Justification for using LSTM . . . . . . . . . . . . . . 63

3.2 .6 Forecasting using LSTM . . . . . . . . . . . . . . . . . 64

3.2.7 Testing and training the data $\ldots \ldots \ldots$. . . . . . . . . 64

3.2.8 Accuracy of forecast results and convergence test . . . . . . . . 65

3.3 Proposed Solution to the AWHS Optimization Challenge . . . . . . . . 66

3.4 Rational for the ancillary services under consideration . . . . . . . . . 67

3.5 Difference between this work and other existing utility programs . . . . . 69

4 Description of Method $\quad 71$

4.1 Optimization Problem Statement . . . . . . . . . . . . . . . 71

4.2 Why 10,000 water heaters? . . . . . . . . . . . . . . . . 71

4.3 Justification for the use of Linear Programming . . . . . . . . . . . 72

4.4 Problem Variables . . . . . . . . . . . . . . . . . . . . . . 76

4.4.1 Time interval index $i \ldots \ldots \ldots \ldots \ldots$ 
$4.4 .2 \quad$ Service Prices . . . . . . . . . . . . . . . . . . 76

4.4.2.1 Peak Demand Price, $a_{i} \ldots \ldots \ldots \ldots \ldots$

4.4.2.2 Frequency Regulation Price, $b_{i} \ldots \ldots \ldots$. . . . . 77

4.4.2.3 Frequency Response Price, $c_{i} \ldots \ldots \ldots$. . . . 78

4.4.3 System Energy Take, $E_{i} \ldots \ldots \ldots \ldots$. . . . . . . . 80

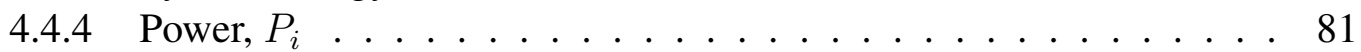

4.4.5 Revenue Earned, $G_{i} \ldots \ldots \ldots \ldots \ldots \ldots \ldots$

4.4.6 Number of units, $N_{i} \ldots \ldots \ldots \ldots$

4.5 Decision Variables . . . . . . . . . . . . . . . . . . . . . . . . . . . 84

4.5.1 Peak Demand Mitigation Energy, $x_{1} \ldots \ldots \ldots \ldots$. . . . 84

4.5.2 Frequency Regulation Energy, $x_{2} \ldots \ldots \ldots \ldots$

4.5.3 Frequency Response Energy, $x_{3} \ldots \ldots \ldots \ldots$. . . . . 86

4.6 Optimization Parameters Definitions . . . . . . . . . . . . . . . 87

4.6 .1 Objective function $\ldots \ldots \ldots \ldots \ldots \ldots$

4.6.2 Optimization Constraints . . . . . . . . . . . . . . . . . . . . . 89

4.6.2.1 Available Power . . . . . . . . . . . . . . . . . 89

4.6.2.2 Available Energy . . . . . . . . . . . . . . . . . 91

4.6.2.3 Peak Demand Mitigation . . . . . . . . . . . . . . . 92

4.6 .3 Optimization Equation . . . . . . . . . . . . . . . . 92

4.6.4 Energy take decay solution . . . . . . . . . . . . . . . 93

4.7 Optimization flow chart . . . . . . . . . . . . . . . . . . . . . 94

5 Results \& Analysis $\quad 96$

5.1 Baseline AWHS overview . . . . . . . . . . . . . . . . . . . . 97

5.2 Single AWHS dispatches $\ldots \ldots \ldots 101$

5.3 Multiple AWHS Dispatches . . . . . . . . . . . . . . . . . . . . 109

5.4 Prolonged AWHS dispatches $\ldots \ldots \ldots 113$

5.5 Data Forecasting and Convergence . . . . . . . . . . . . . . . 121

5.5 .1 Energy Forecasting . . . . . . . . . . . . . . . . 121

5.5 .2 Price Forecasting . . . . . . . . . . . . . . . . 140

5.5 .3 Weather Forecasting . . . . . . . . . . . . . . . 146

5.6 Optimization of the AWHS . . . . . . . . . . . . . . 150

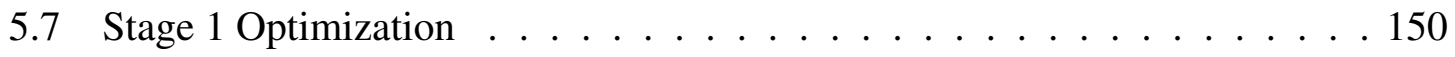

5.7.1 Case 1.1 - Limiting services . . . . . . . . . . . . . . . 151

5.7.2 Case 1.2 - Stage 1 Optimization . . . . . . . . . . . . 153

5.7.3 Case 1.3 - Multi-service reservation . . . . . . . . . . . 157

5.7.4 Case 1.4 - Bounding Frequency response price variations . . . . . 159

5.7.5 Case 1.5 - Setting the upper boundary of frequency regulation . . 163

5.7.6 Case 1.6 - Bounding Peak Demand Mitigation with variation in frequency response prices . . . . . . . . . . . . . . . 165

5.8 Stage 2 Optimization . . . . . . . . . . . . . . . . . . . . . . . . . . . . . . . 169

5.8.1 Case 2.1 - 24 hour continuous dispatches with no upper limit boundary set on frequency regulation . . . . . . . . . . . . . 169 
5.8.2 Case 2.2 - 24 hour continuous dispatches with an upper limit boundary set on frequency regulation . . . . . . . . . . . . . 171

5.8.3 Case 2.3 - Continuous dispatches over prolonged durations on a non-peak demand day . . . . . . . . . . . . . . . . 173

5.8.4 Case 2.4 - Dispatch for Frequency Response . . . . . . . . . . . 176

5.9 Analysis of Revenues . . . . . . . . . . . . . . . . . . 180

5.10 What would the value be of including other ancillary services? . . . . . . 182

5.11 Choice of Linear Programming constraints . . . . . . . . . . . . . . 183

5.12 What would other objectives have been? . . . . . . . . . . . 183

5.13 Shortcomings of this work $\ldots \ldots \ldots \ldots$

6 Conclusion 185

$\begin{array}{lll}7 & \text { Future Work } & 187\end{array}$

$\begin{array}{ll}\text { Bibliography } & 191\end{array}$

Appendix A: MATLAB ${ }^{\circledR}$ Implementation of AWHS Optimization, stage $1 \quad 214$

Appendix B: MATLAB ${ }^{\circledR}$ Implementation of Test and Training using LSTM 219

Appendix C: MATLAB ${ }^{\circledR}$ Implementation of AWHS Optimization, stage 2 


\section{List of Figures}

1.1 Frequency event captured at PSU's Power Lab in September 2019. . . . . . . . 17

1.2 Electric Water heater response times to Load and Shed commands [1]. . . . . . 19

1.3 CAISO's Duck Curve [2]. . . . . . . . . . . . . . . . . . . . . . 20

1.4 Average Daily Residential Load for Total Demand and Water Heating (Pacific Northwest) [3]. . . . . . . . . . . . . . . . . . 21

3.1 Optimization Method Summary. . . . . . . . . . . . . . . 58

3.2 LSTM test and training flow diagram. . . . . . . . . . . . . . 65

4.1 Frequency response events recorded at the Salem Smart Power Center from August 23, 2019 - January 31, 2020, Source: PGE. . . . . . . . . . . . . . . 79

4.2 State diagram for electric water heater model [4] . . . . . . . . . . . . . 81

4.3 Maximum power available in the AWHS vs. the number of consecutive fiveminute dispatches [4] . . . . . . . . . . . . . . . . . . . 82

4.4 AWHS optimization process flow chart. . . . . . . . . . . . . 95

5.1 Load profile for a typical day with no AWHS resource dispatch. . . . . . . . . 98

5.2 Load profile for 5 days with no draw . . . . . . . . . . . . . . . 100

5.3 Onetime, $5 \mathrm{MW}$ draw for 5 minutes at 0200 hour (1.7 MW-hr). . . . . . . . . . 102

5.4 Percent of units heating, $5 \mathrm{MW}$ draw for 5 minutes at 0200 hour. . . . . . . . . . 104

5.5 A onetime, $20 \mathrm{MW}$ draw for 60 minutes at 0200 hour (20 MW-hr bid). The AWHS system runs out of energy. . . . . . . . . . . . . . . 106

5.6 Percent of units heating, $20 \mathrm{MW}$ draw for 60 minutes at 0200 hour. . . . . . . . 108

5.7 Three $12 \mathrm{MW}$ draw for 5 minutes each. The system begins to recover after each draw before the next draw, starting at 0100,0200 and 0300 . . . . . . . . . 110

5.8 The percentage of units turned on corresponding to the three five minute bids. . 112

$5.91 \mathrm{MW}$ draw continuously for 13 hours, from 0300 to 1330 . . . . . . . . . . 114

$5.101 \mathrm{MW}$ continuously for 24 hours, i.e, $12 \mathrm{MW}-\mathrm{hr}$ for 24 hours. . . . . . . . . . . 116

5.11 Separate hourly $1 \mathrm{MW}$ dispatch from $0300 \mathrm{hr}$ to noon for recovery time observations. . . . . . . . . . . . . . . . . . . . 118

5.12 Ten 1 MW-hr dispatches, with a projection into the next 24 hours. . . . . . . 120

5.13 one year's worth days worth of energy take data. . . . . . . . . . . . 123

5.1428 days worth of energy take data. . . . . . . . . . . . . . . . . . . . . . . . . . . . . . . . . . .

5.1510 days worth of energy take data. . . . . . . . . . . . . . 127

5.16 July $1 \&$ July $2,75 \%$ training data, 25\% test data, 100 iterations. . . . . . . . . 129 
5.17 July 1 \& July $2,75 \%$ training data, $25 \%$ test data, 200 iterations. . . . . . . . 131

5.18 July 1 - July 5, 80\% training data, 20\% test data, 100 iterations. . . . . . . . . 133

5.19 July 1- July 10, training data, August 11 forecasted test data, 100 iterations. . . 135

5.20 July 1- July 10, training data, August 11 forecasted test data, 200 iterations. . . 137

5.21 RMSE for Energy take prediction is less than 5\% . . . . . . . . . . . . 139

5.22 Historical three week LMP pricing data. . . . . . . . . . . . . . . . . . . . . . . . . . . . . . .

5.23 LMP Pricing for 7 days look-ahead forecast. . . . . . . . . . . . . . . . 143

5.24 RMSE for LMP prediction is less than 5\% . . . . . . . . . . . . . . . . . . 145

5.252012 Winter weather data, minimum temperatures ranging from $29^{\circ} \mathrm{F}$ to $31^{\circ} \mathrm{F}$. source: NOAA. . . . . . . . . . . . . . . . . . . . . . 147

5.262012 Summer weather data, max. temperature per day ranging from $90.5^{\circ} \mathrm{F}$ to $100^{\circ} \mathrm{F}$ source: NOAA. . . . . . . . . . . . . . . . . . . 149

5.27 Stage 1- $1 \mathrm{MW}$ reserves placed on 3 services. . . . . . . . . . . . . . . . . . . . . . . . . . . . . .

5.28 Stage 1 optimization of all services. . . . . . . . . . . . . . . . . 154

5.29 Stage 1 optimization of all services. . . . . . . . . . . . . . 155

5.30 Stage 1 optimization of all services. . . . . . . . . . . . . . 156

5.31 Stage 1- multi-service reservation. . . . . . . . . . . . . . . . 158

5.32 Stage 1- Lower frequency response price of $\$ 20 \ldots \ldots$. . . . . . . . . . 160

5.33 Stage 1- Higher frequency response price of $\$ 40 . \ldots 162$

5.34 Stage 1- Setting the upper boundary of frequency response. . . . . . . . . . . . 164

5.35 Stage 1- Bounding PDM to $1 \mathrm{MW}$ per 5 minute interval with a frequency response price of $\$ 20 \ldots \ldots$. . . . . . . . . . . . . . 166

5.36 Stage 1- Bounding PDM to $1 \mathrm{MW}$ per 5 minute interval with a frequency response price of $\$ 40 \ldots \ldots$. . . . . . . . . . . . . . . . 168

5.37 Stage 2 Optimization- AWHS fails:runs out of energy take around time interval 115. . . . . . . . . . . . . . . . . . . 170

5.38 Stage 2 Optimization- 0.17 MW-hr dispatched successfully without running out of energy take. . . . . . . . . . . . . . . . . . . 172

5.39 Stage 2 Optimization- Dispatch profile on a non-peak day. . . . . . . . . . 175

5.40 AWHS profile with no frequency response. . . . . . . . . . . . . . . . 177

5.41 AWHS profile with frequency response event. . . . . . . . . . . . . . . . 179 


\section{List of Acronyms}

AA Asset Aggregation

ACE Area Control Error

AWHS Aggregated Water Heater System

BESS Battery Energy Storage Systems

BPA Bonneville Power Administration

CAES Compressed Air Energy Storage

CAISO California Independent System Operator

DC Direct current

DER Distributed Energy Resources

DHS Discrete Harmony Search

DLC Direct Load Control

DSG Dispatchable Standby Generation

DSM Demand Side Management 
EMS Energy Management Systems

ESS Energy Storage Systems

EVs Electric Vehicles

EWH Electric Water Heaters

FASTADR Fast Automated Demand Response

HBB-BC Hybrid Big Bang-Big Crunch

HES Hybrid Energy Systems

HVAC Heating Ventilation and Air Conditioning

IoT Internet of Things

ISO Independent System Operator

LBNL Lawrence Berkely National Lab

LCOE Levelized Cost of Electricity

LMP Locational Marginal Pricing

LP Linear Programming

LPSP Loss of Power Supply Probability

LSTM Long Short Term Memory 
MISO Mid-Atlantic Independent System Operator

MOEA Multi-Objective Evolutionary Algorithms

NERC North American Electric Reliability Corporation

NOAA National Oceanic and Atmospheric Administration

NREL National Renewable Energy Laboratory

OEM Original Equipment Manufacturer

OPENADR Open Automatic Demand Response

PGE Portland General Electric

PHS Pumped Hydro Storage

PJM PJM, formerly Pennslyvania, New Jersey and Maryland ISO

PSO Particle Swarm Optimization

PV Photovoltaic

RER Renewable Energy Resources

RMSE Root Mean Square Error

RNN Recursive Neural Networks

SVF Solar Valley Filling 
TGR Traditional Generation Resources

VPP Virtual Power Plant 


\section{Introduction}

\subsection{Background}

Until recently, electricity generation and supply were usually centralized. Street lights, industrial machines and household electrical appliances were powered from a far away, remote location. Since the invention of Thomas Edison's Direct current (DC) light bulb in the late 1880's and the century that followed, electrical customers were content with the status quo, and only thought of their utility provider once a month when they paid their electric bills, or when service gets interrupted.

Towards the end of the twentieth century however, electricity consumers started generating a portion of the power they used locally. Advancements in manufacturing technology and the relative ease of installation and deployment have enabled Photovoltaic (PV) systems to become more economical. Backed by many governmental incentives in the form of tax credits to homeowners and a plethora of rebates for both residential and industrial sectors alike, acquisition of grid-connected PV systems have recently become easier to acquire. The wind energy industry has experienced its own revolution. PV and wind are forms of Renewable Energy Resources (RER) that provide energy to meet the utility industry's day-to-day energy demand.

RER are becoming less expensive and widely adopted. They help minimize dependence 
on fossil fuels. However, for all the benefits afforded the electric utility industry by RER, they create unintended and adverse effects on the electric grid.

These adverse effects results largely from the stochastic nature of RER and the negative impact of excessive RER generation on power system reliability and grid resiliency. Such challenges cause distribution transformer overload, transmission congestion, excessive curtailment and unfair energy pricing of Traditional Generation Resources (TGR). Some of these problems can be addressed by the use of Energy Storage Systems (ESS), Dispatchable Standby Generation (DSG), Demand Side Management (DSM), and Hybrid Energy Systems (HES). Although the use of Battery Energy Storage Systems (BESS) is the most promising of all, this technology is still in its infancy. In this work however, we focus our attention to the use of an Aggregated Water Heater System (AWHS) as a new way to provide multiple ancillary services for the purposes of relieving the grid of the adverse impacts of RER.

\subsection{Research Objective}

The primary objective of this work is to find a better way to reduce the negative impact of RER on the electrical grid by using large aggregations of residential water heaters to provide ancillary grid-support services.

\subsection{Research Question}

When aggregated, can electric resistive water heaters be used to alleviate the negative impacts of RER on the electrical grid by economically providing ancillary grid-support 
services?

\subsection{Problem Statement Summary}

The problem that this dissertation is trying to solve is how to alleviate the negative impacts of RER on the grid by optimally dispatching the AWHS for ancillary services in a manner that maximizes revenues.

\subsection{Problem Statement question}

How should a water heater load resource be allocated between three ancillary services, frequency regulation, frequency response, and peak demand mitigation, at any given time of day based on the available energy among 10,000 water heaters in such a way that dispatch is optimized to maximize revenue? Revenue is maximized by the determination of the optimal ratio of ancillary services to provide based on market price signals.

\subsection{Significance of the proposed solution}

About 55\% of homes in the Pacific Northwest had an Electric Water Heater (EWH) in 2017. This equates to 3.3 million households, using 2017 U.S census numbers. According to Bonneville Power Administration (BPA), by 2020, only about 4,000 water heaters in the Pacific Northwest may become network enabled. [5]

The significance of this work is that our research can help justify the need for Original Equipment Manufacturers (OEM) to ship traditional water heaters with network enabled 
communication protocols like CTA2045, or other industry-wide, universal open source control platforms like OPENADR or IEEE 2030.5.

In addition, the evidence gathered from this research can be used to inform a future when water heaters becomes revenue centers, by providing financial benefits to home owners for acting as participants in the ancillary services energy market.

\subsection{Details of the Problem}

The proliferation of RER like PV and wind power is presenting economical, operational and systemic challenges to electric utilities, energy balancing authorities, market dispatchers, and to the aging electric grid at large.

PV and wind power are forms of RER that provide energy that can be used to meet the utility industry's day-to-day demand. RER are power generation resources that are becoming less expensive and widely adopted. They also help minimize dependence on fossil fuels. However, they can sometimes create unintended and adverse effects on the electric grid. We detail the negative impacts of RER on the electric grid in the following sub-sections.

\subsubsection{Stochastic nature of RER}

RER are inherently weather dependent. They often produce rapid changes in power correlated with weather conditions, resulting in unscheduled ramping events within utility balancing areas. These ramping events present scheduling challenges for utilities operating within hourly or sub-hourly electricity trading markets [6-8]. For instance, uncertainty regarding the forecast of wind ramping events, specifically the timing and ramp rates, affects 
energy dispatchers' options for maintaining balance between electricity supply and demand, which is measured using the Area Control Error (ACE). Utilities that allow their ACE to deviate outside of the permitted defined limits may face significant fines from regulating authorities. Consequently, the unpredictable nature of RER directly impacts most electricity marketers' ability to readily and easily satisfy their energy supply and delivery contracts $[9,10]$.

\subsubsection{RER depress overall energy prices}

In an effort to boost adoption rates, RER have been heavily subsidized by local, state and federal government agencies. In 1998, the federal subsidy for wind energy amounted to about $\$ 5$ million. However, by 2012 , these subsidies had passed the $\$ 1$ billion mark. ${ }^{1}$ According to a Congressional Research Service (CRS) study, between 2016 and 2020, energy-related tax expenditure regarding wind production tax credits will cost the treasury $\$ 25.7$ billion. $^{2}$ In the United States, PV and wind receive more than fifty times more subsidies for each megawatt hour produced than TGR like fossil fuel generators. Some have argued that the subsidy model of RER is depressing Locational Marginal Prices (LMP) of energy on an hourly and monthly basis, causing lower overall revenue and revenues for TGR. $^{3}$

\footnotetext{
'Tax-Blowing Boondoggle Don't Give Wind Energy More Credit Than It Deserves', www.forbes.com/sites/larrybell.html

2 'Wind Subsidies Should End',https://www.instituteforenergyresearch.org/analysis.html

3 'Is renewable energy threatening power reliability?',www.utilitydive.com/news/is-renewable-energythreatening-power-reliability/443701/
} 


\subsubsection{Impact on the resiliency and reliability of the grid}

According to a number of sources, the current growth rate of RER is unsustainable [11, 12]. If trends continue, grid reliability and resiliency will be put at risk [13]. Installation and inter-connection need to be properly planned and strategically deployed [11].

The electric grid consists of the distribution system $(4.12 \mathrm{kV}$ to $26 \mathrm{kV})$ and transmission system ( $26 \mathrm{kV}$ up to $765 \mathrm{kV}$ ). The voltage and connection points of RER differ based on the technology, manufacturer, year installed, location on the grid, and distance from the load served. These variations can make system integration very challenging. Wind farms are located in rural areas where there are significant wind resources. These are are usually connected to the transmission system. PV on the other hand, can either be connected to the distribution or transmission system depending on if the PV system is residential or utility-scale.

Further, production fluctuations make transmission and system planning difficult to forecast, because it is impossible to accurately predict and model solar irradiance or wind speed levels. More so, since the energy output of these RER can not be economical stored at a large scale, system operators and grid dispatchers are often forced, around the clock, to schedule and dispatch TGR and other resources around the highly variable production levels of RER. 


\subsubsection{Large scale adoption}

There is also the problem of scalability. Residential PVs are sparsely distributed, injecting power into the grid at different, usually unplanned tie-in points. Traditionally, system planning is mostly engineered based on reliability factors like customer load, voltage drop concerns, system protection and frequency regulation. Recently, with little control of where, and how many RER are installed within a residential district or distribution loading center, the rate of adoption of RER like residential PV has caused grid reliability and resiliency concern for some system operators. For instance, with about $12 \%$ of its residential homes mounted with PV solar panels, in 2016, the Hawaiian Electric Company (HECO) halted thousands of application requests to connect customer-owned solar panels to its electric distribution system, citing concerns of overwhelming the State's island power grids. The Solar Energy Industry Association (SEIA) estimates that in Hawaii alone, the number of installed systems will exceed three million (more than double the State's 2017 estimated population of 1.43 million). ${ }^{4}$ States like Arizona and California, and Countries like Japan and Germany have expressed similar concerns. ${ }^{5,6}$

\subsubsection{Reduction in power system inertia}

Most TGR have high inertia [14]. The electrical grid is comprised of many interconnected TGR. Changing grid frequency is akin to changing the inertia of every synchronous generator

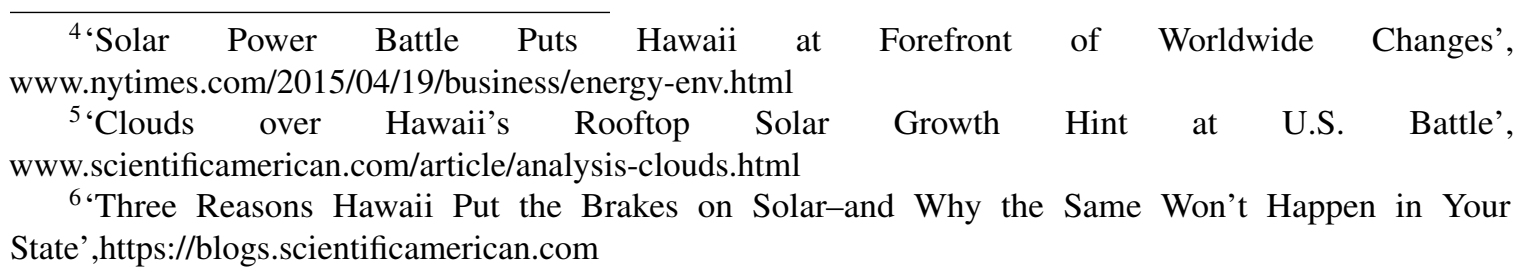


connected to the grid. This inertia helps slow down large impacts to the grid, and buys system operators a few more seconds in response time. As more RER replace TGR, the total system inertia in the grid is being replaced with power electronics-based frequency regulators that are built into RER, which do not have mechanical inertia.

Per UL 1741-1999, PV inverters shall have a system frequency error of less than $2 \%$, and for frequency measurements at the inverter, the error shall be less than $0.1 \mathrm{~Hz}$. According to IEEE 1547, a stable frequency is required of all devices connected to the grid else the inverter must disconnect from the grid if its frequency becomes unstable. In order to maintain a constant frequency on the grid, the active power supply must be equal to the load at any given time. TGR uses a generator governor to constantly match the load and generated output power. With PV and wind largely dispersed geographically and very different from system to system (depending on the technology type, manufacturer, location, model year and entry voltage into the grid), the act of matching load to generation in order to regulate the system frequency with less inertia in the grid has become more challenging for system operators.

\subsubsection{Transmission congestion}

Transmission congestion occurs when least-cost generated power cannot reach a load due to inadequacy of the transmission network to safely and reliably deliver power [15]. When transmission capacity fails to track growth in peak electric load, the transmission system experiences congestion [16]. 
RER cause transmission congestion largely due to their unpredictable nature. For instance, congestion can arise due to power ramp up at night when wind speed picks up. Excessive production, especially when not needed by the grid, can be a burden on transmission lines.

Most of the United States' electrical transmission infrastructure was built in the 1950's and 1960's. As these transmission lines age and loading increases (either due to population growth or increased industrialization and urbanization), their thermal limits and maximum allowable ampacities decreases. While many transmission lines can reach their peak load ampacities at certain points in time, these peak ampacity requirements are not constant. The result is that planning for new transmission lines that are more reliable is more challenging than ever due to the stochastic nature and system requirements of RER, which can sometimes be difficult to estimate with an acceptable degree of precision.

\subsubsection{Curtailments}

Until recently, RER have not been required to provide frequency support services. Wide fluctuations and swings in the power produced by PV and wind power can cause severe frequency stability problems to the electrical grid $[17,18]$. In addition, because RER lack inertia and are not mechanically synchronized like TGR, an increase in the penetration of RER can cause frequency imbalance on the grid [19]. Hence during periods of high power production, RER, especially wind, must be curtailed due to excess production or grid stability concerns. Curtailments usually happen during periods of high RER production. In other situations when RER unexpectedly stops production, TGR, usually peaker power 
plants, are quickly brought online to help stabilize the grid, putting further stress on existing TGR and the electric grid at large.

\subsection{Available Solutions to the Problem and Current Practices}

A number of solutions to this problem already exists. The benefits of these solutions have been demonstrated in both literature and practice by researchers and electric utility companies globally. Five prominent solutions are described in this section, namely, Energy Storage Systems (ESS), the use of TGR for providing ancillary services, Dispatchable Standby Generation (DSG), Hybrid Energy Systems (HES), Demand Side Management (DSM), and Asset Aggregation (AA).

\subsubsection{Energy Storage Systems (ESS)}

ESS store excess energy for use at a later time. ESS include Battery Energy Storage Systems (BESS), Pumped Hydro Storage (PHS) and Compressed Air Energy Storage (CAES), all of which are currently used by utilities and energy providers to store the excess energy generated by RER, although with varying degrees of technological maturity.

BESS are electrochemical systems that manage energy storage through alteration of battery cell chemistry. BESS are charged (energy is stored) during off peak periods, when the price of electricity is cheap, and then discharged (the stored energy is released for use) at a later time. 
PHS store energy in the form of the potential energy of water that has been pumped to a higher elevation. Pumping occurs when electricity prices are low. Water is then released and passed through the pumps, now acting as turbines, when energy costs are high.

CAES store energy as compressed air. This stored pressure-volume energy is then released at a later time when called upon to generate electricity, such as when energy prices have risen.

BESS are not yet very mature . According to Bloomberg, the total storage capacity of all batteries in the United States as of 2018 is less than $1 \%$ and the total storage capacity is estimated to increase to only $3 \%$ by $2040 .{ }^{7}$ However, a lot of research is being conducted in the area of storage [20-23].

The Public Utility Research Center, based on a comprehensive data analysis and research, outlined the impacts of lower natural gas prices, declining prices for renewable technologies, regulatory policies, and laws that are influencing the increase in demand for utility-scale energy storage [20]. Barbose presented a status update on the role of Renewable Portfolio Standards (RPS) in the United States and the impact on the growth of Energy Storage is presented [21]. This report was developed in collaboration with the Lawrence Berkley National Lab and the U.S Department of Energy. Wang et al. evaluated the reliability benefit of energy storage to systems consisting of multiple renewable sources considering their operating strategy [22]. The reliability evaluation models of energy storage that can be used in Monte Carlo simulations with other forms of RER are developed. The inherent

\footnotetext{
${ }^{7}$ Bloomberg, www.bloomberg.com/news/articles/2016-03-17/the-big-battery-boom-hits-anotherroadblock-fire-fearing.html/"
} 
characteristics that are deterministic to the chronological variation of power output are incorporated when modeling, including storage factors like energy conversion performance and charge/discharge constraints of energy storage.

An extensive literature review is presented by Diaz-Gonzalez et al., highlighting several energy storage technologies, particularly for wind power applications. The review presents the main operating principles, the characteristics of energy storage technologies suitable for stationary applications. The definition and discussion of potential energy storage applications was thoroughly covered in [23].

With over $150 \mathrm{GW}$ globally, PHS represents around 99\% of the world's electrical energy storage capacity. Currently, Japan is the worldwide leader in PHS, but China is expanding quickly and was expected to surpass Japan by the end of 2018 [24, 25]. In early 2019, it was reported that China had surpassed Japan and that China's PHS installations accounted for $78 \%$ of the world's new PHS installations accounting for more than $2 \mathrm{GW}$ of newly commisioned units (in 2018 alone) and a cumulative $29 \mathrm{GW}$ total. ${ }^{8}$ A major issue with PHS is the limited number of viable sites, which must have both a water resource and sufficient elevation gains [26]. Other problems include available storage volume, acceptable charge and discharge rates, and environmental siting issues. The overall system efficiency may decrease if the rates and storage volume requirements are not met [27, 28].

CAES also has its own drawbacks, such as energy loss due to excessive heat dissipation during the compression of air and air leakage from CAES caverns. CAES sites are also

\footnotetext{
${ }^{8}$ Asian Power, www.asian-power.com/power-utility/news/china-accounted-78-worlds-new-pumpedstorage-hydropower-installations
} 
limited, as they must be located near caverns that can reliably contain compressed air, such as salt domes [29, 30].

Storage can be cost prohibitive, and as a result, despite its prospects, storage in general, regardless of its technology type, has not yet become main stream.

\subsubsection{Use of Dedicated TGR for Ancillary Services}

Another solution to the problems created by RER is the use of dedicated TGR to provide ancillary services in order to accommodate the stochastic nature of RER. TGR include coal, nuclear, hydro, and natural gas power plants.

Most TGR are designed to provide base load power, although they can be equipped with enhanced technologies, and operated with improved operational practices that allow for more flexible dispatch to provide additional grid support services. With the increased penetration of RER in recent years, new TGR power plants are being built specifically for grid support ancillary services rather than for base load generation. For example, because some varieties of natural gas plants can be quickly ramped up and connected to the grid in a mater of minutes, they are suitable for providing fast-response ancillary services, which are often needed during peak hours when demand is very high and the sun is beginning to set [31].

As the penetration of renewables continues to increase, cheaper RER continue to displace conventional TGR, resulting in short dispatch durations and frequent cycling of TGR [32]. These factors can lead to higher TGR operation \& maintenance costs. Consequently, some utilities have assigned specific power plants for some ancillary services, as long as 
the subject plants can meet the minimum requirements for participation in that particular ancillary services market. Building new TGR is a very expensive undertaking and can take many years to complete. Furthermore, many approvals and revisions are required from public utility commissions, public agencies, and regulatory authorities [33].

The problems caused by RER can not be fully comprehended without a proper understanding of what ancillary services are, and why they are important to electricity supply and demand. In an effort to avoid confusion, in 1995, the Federal Energy Regulatory Commission (FERC) issued a definition of ancillary services as those "necessary to support the transmission of electric power from seller to purchaser given the obligations of control areas and transmitting utilities within those control areas to maintain reliable operations of the interconnected transmission system". Simply put, ancillary services are services necessary for continuous uninterrupted operation of the grid. These services ensure the quality of energy delivered through the electrical grid [34]. These services include but are not limited to:

\subsubsection{Frequency Regulation}

Often referred to as 'regulation', frequency regulation is a set of tools or actions executed either automatically or manually by power system operators that ensures a steady frequency $(60 \mathrm{~Hz})$ during situations or events that causes the system frequency to become too high or too low. There are three types of frequency regulation responses; primary, secondary and tertiary. Both primary and secondary regulation are triggered automatically within seconds and tens of seconds respectively. Tertiary regulation is initiated by the operator within a few 
minutes if both primary and secondary regulation are not sufficient to restore the frequency to $60 \mathrm{~Hz}$.

\subsubsection{Frequency Response}

Frequency response is the measure of a system's output to an input signal of varying frequency. Frequency response is measured in decibel $(\mathrm{dB})$, with the phase, measured in radians ${ }^{9}$. For power systems however, we chose the definition provided by the North American Electric Reliability Corporation (NERC). According to NERC, frequency response is a balancing area's ability to stabilize grid frequency immediately following the sudden loss of generation or load.

The distinction between frequency regulation and frequency response is that frequency regulation addresses slight and slower frequency deviations while frequency response addresses large and fast deviations.

The ability of generation resources to respond to frequency deviation events is critical to the reliability of the electric grid. Frequency response may be called upon for events such as a sudden loss of generation, start-up of large industrial loads, or a power outage caused by weather storms, substation fire, or electrical equipment failure. Simply stated, frequency response is an immediate, and automatic response to changes in grid frequency caused by grid events.

TGR and very large industrial motors and pumps supply inertia to the grid that helps reduce the impact of frequency fluctuations on the grid. Due to the advent of variable

\footnotetext{
${ }^{9}$ http://www.digitizationguidelines.gov/term.php?term=frequencyresponseaudio
} 
frequency drives, RER like wind and solar, and the continuous retirement of synchronous TGR, there has been a continued decline in the amount of resources available to support frequency response. Though, recent technology has enabled solar and wind to have some frequency response capability embedded.

Frequency response is not currently a compensated electricity market product. But, system operators are required to respond to frequency deviations within their service territory. Ideally, one would expect that all TGR in the system provide frequency response services, but this requirement can be cost prohibitive, and large frequency deviations are not very common. Frequency response events are very fast, requiring very quick power injection into the grid to arrest the frequency deviation. In Figure 1.1, we show a frequency response event that was captured at Portland State University (PSU) power lab in September of 2019. 


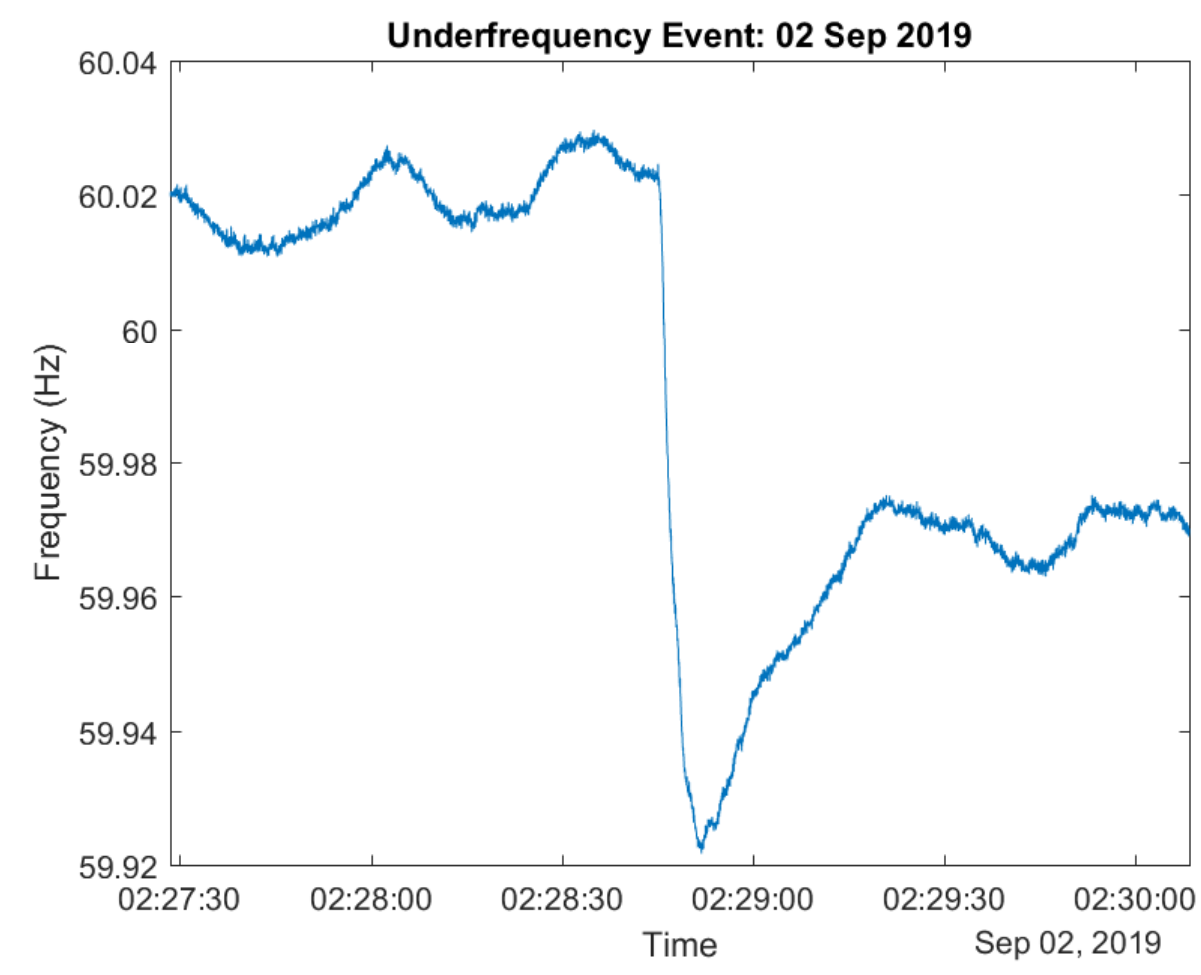

Figure 1.1: Frequency event captured at PSU's Power Lab in September 2019.

Reserving TGR that could otherwise be used for capacity and other grid market services for frequency response is not very economical. Further, because frequency deviations are stochastic, it can be difficult to assess how much frequency response resource is needed. RER like wind farms and PV solar plants are mostly operated at or near their maximum capacity with no room for participation in frequency response.

In a 2017 docket, the Federal Energy Regulatory Commission (FERC) [35] noted a decline in frequency responses in the North American grid due to the fact that between 2011 and 2014, 42 GW of synchronous generating plants were retired. According to an Energy Information Administration (EIA) report, $14 \mathrm{GW}$ of coal and $3 \mathrm{GW}$ of natural gas-fired plants were retired in 2015 [36]. For example, Portland General Electric plans to retire its 
550 MW Boardman Coal plant in 2020, the only coal plant in its generation portfolio.

In its response to FERC, the Energy Storage Association (ESA) highlighted that Electricity markets would be less efficient and system costs will be higher than necessary if generation resource owners are required to provide frequency response service from generators more suited to provide energy and capacity [37]. Further, requiring generators to provide frequency response could produce an expensive but costly oversupply of frequency response headroom. Not all TGR resources are well positioned to provide frequency response, and by responding to frequency response events, operational efficiency can be impacted. A reduction in operational system efficiency will lead to higher system operational costs.

The AWHS is well suited to provide frequency response, because electric resistance water heaters are fast responding and do not have any headroom reservation requirement. Figure 1.2 illustrates the time intervals between the load and shed commands and the time the water heater actually turns on as 2.5 seconds and 9.0 seconds respectively, both durations fast enough to qualify for frequency response events [1]. 
EWH Load Up Response Time

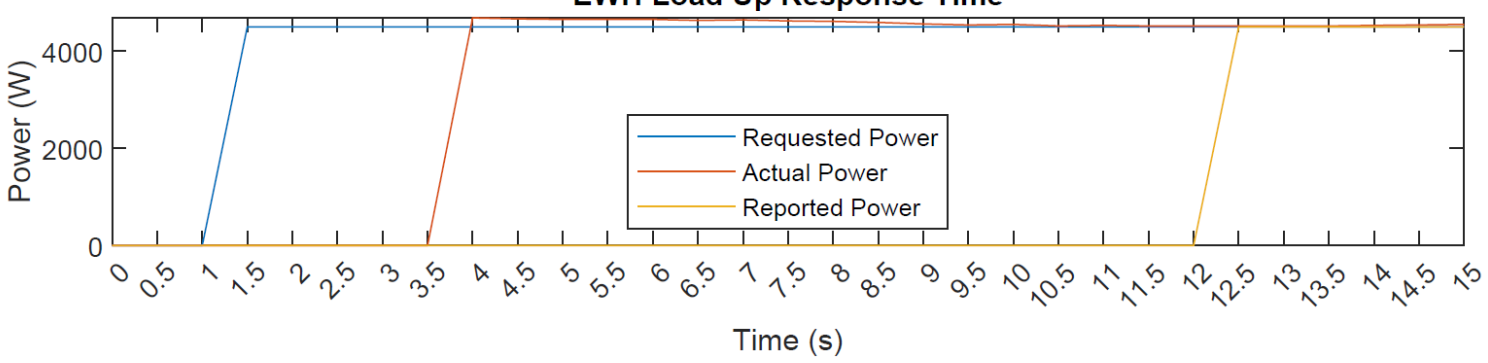

EWH Shed Response Time

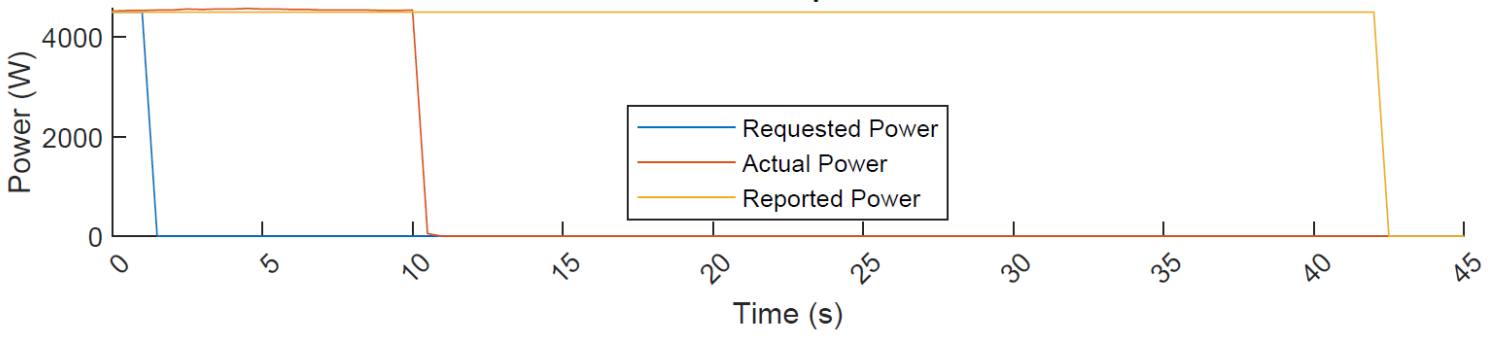

Figure 1.2: Electric Water heater response times to Load and Shed commands [1].

\subsubsection{Peak Demand Mitigation}

Peak demand is a measure of how much power the grid must produce when called upon during periods of high demand. Peak demand occurs when many high energy consuming loads (like air conditioners, large motors, industrial pumps) are all running at the same time, which occurs during peak hours.

Many utilities define peak demand hours as the period between 4:00pm and 8:00pm on weekdays. Peak demand is a proxy measure of the amount of stress put on the grid. Fast ramping, dedicated peaker TGR are usually dispatched to help curtail excess demand during peak hours, as a means of ensuring system stability and reliability. Peak demand mitigation is the process of reducing load (consumption) in the electricity distribution network, during peak hours, thereby limiting the need for peaker TGR. 
Generation from RER like PV decreases as the sun goes down. Consequently, they are unable to adequately contribute to the grid when demand increases during the peak demand hours that occur following sunset. During this period, TGR must ramp their generation from conventional generation plants to meet this surge in demand and compensate for the loss of solar generation. The California Independent System Operator (CAISO) uses the duck curve, shown in Figure 1.3, to illustrate the impact of PVs on the electric grid's operations based on CAISO's real-time analysis and forecast of electricity net demand from 2012 to 2020. The net demand load represents the amount of conventional generation plants (excluding renewables) that will need to be on-line during different times of the day.

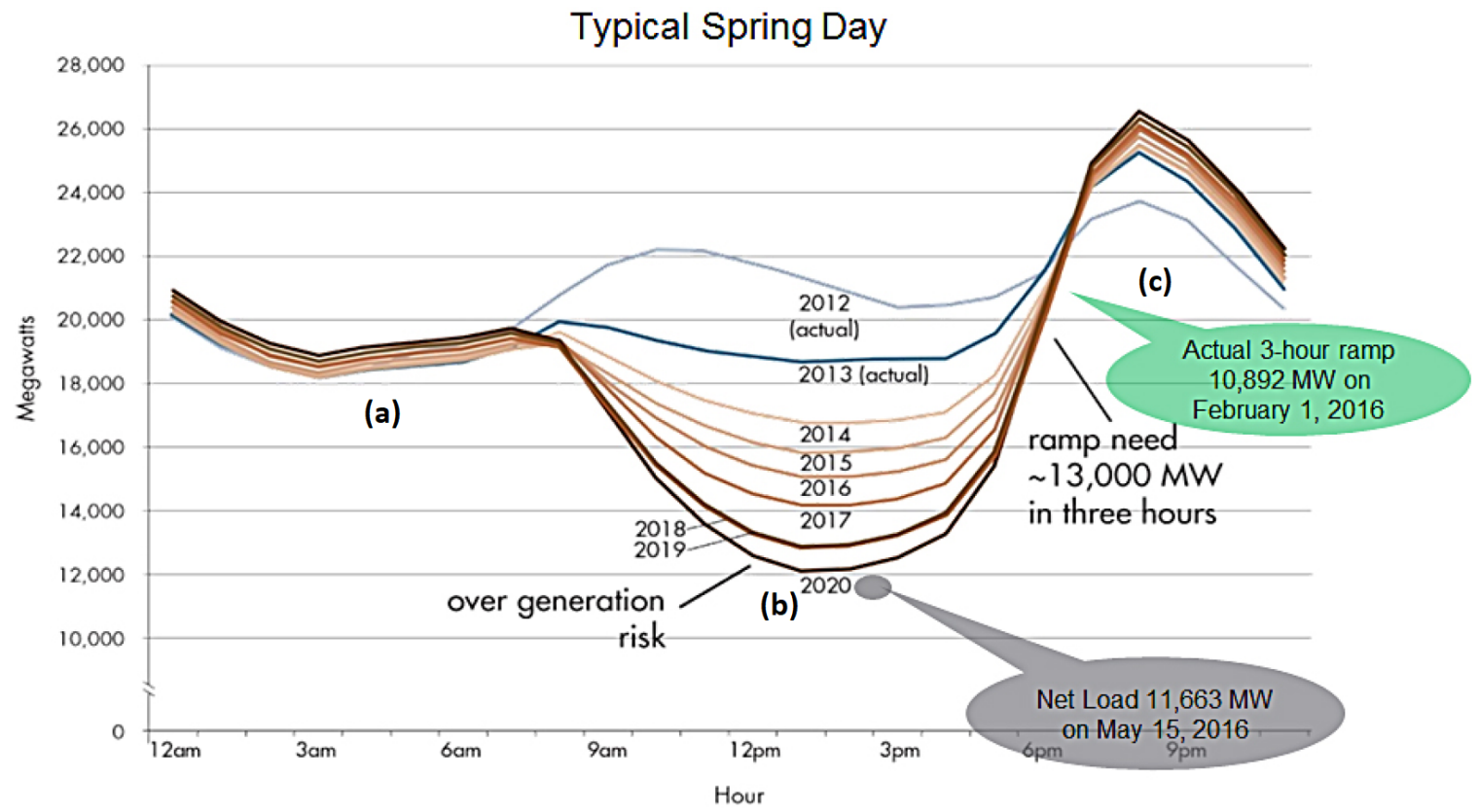

Figure 1.3: CAISO's Duck Curve [2].

Zones (a) and (b) in the duck curve represents the morning and mid-day net demand loads, respectively. Zone (b) has the least predictability because the increase in the generation from PV will cause a reduction in conventional generation. There is an increased risk of over 
generation in this zone. Finally, towards the end of the day, zone (c) is where conventional plants will see the most stress. The unpredictability of DG resources (particularly solar and wind) means that utility companies may not be able to properly control and plan for the variable system electricity demand.

Utilities bill for electricity charges by adding the actual energy consumption (kWh) to the electricity demand charges $(\mathrm{kW})$. Demand charges are based on the highest power provided to customers, in any given fifteen minute period, over the monthly billing period. According to CAISO, steep power ramps, over-generation and the resulting impact on frequency response will need to be quickly addressed as more PV are installed, commissioned and connected to the grid [38].

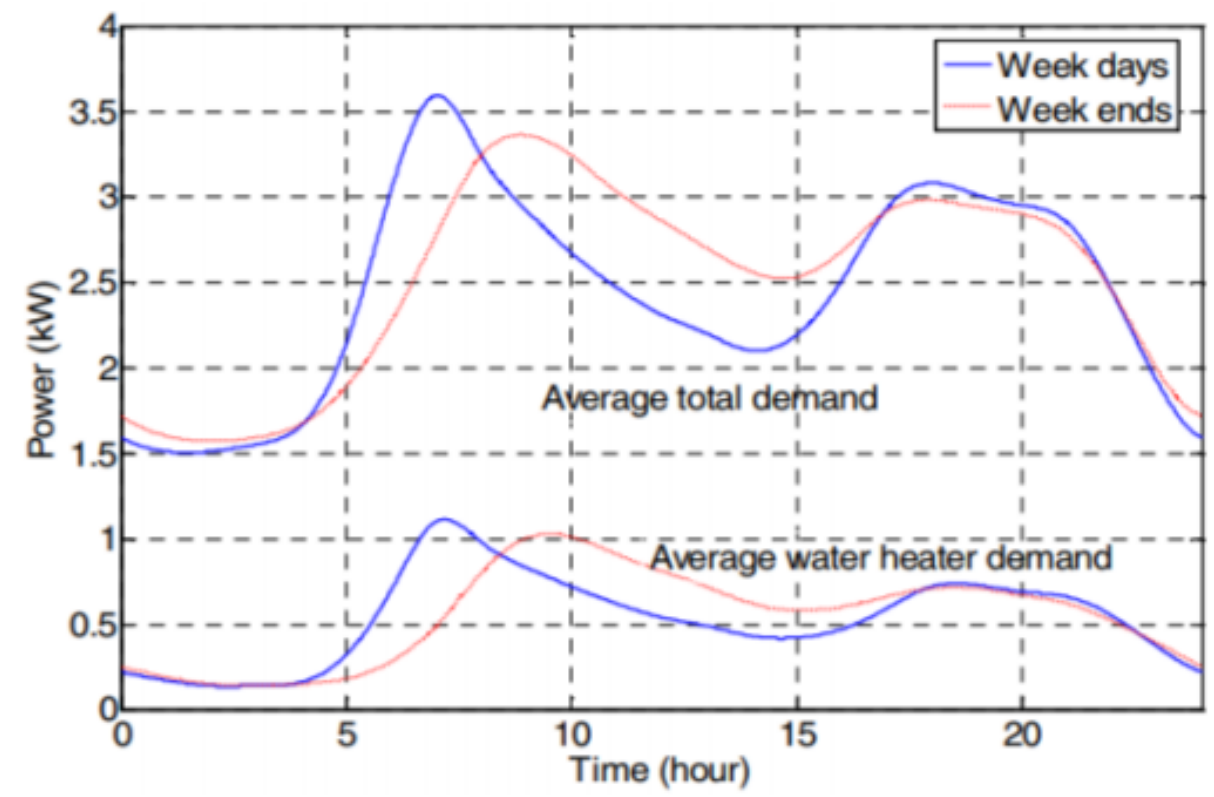

Figure 1.4: Average Daily Residential Load for Total Demand and Water Heating (Pacific Northwest) [3].

Figure 1.4 illustrates that in the Pacific Northwest, water heater usage duration overlaps with the total average electricity peak demand, suggesting that water heaters could be used 
as a tool for peak demand mitigation [1].

\subsubsection{Ramp Rate Control}

Ramp rate control is defined as the ability to control the change in power output of a generator as it is ramping up or down. According to NERC, all resources on the grid must be able to reach their full capacity within 10 minutes of interconnection. Controlling ramp rate is important for grid stability since generation must match load at all times.

\subsubsection{Voltage/Reactive power (VAr) compensation}

Loads like induction motors of household appliances, air conditioners, industrial equipment, and power transformers all consume reactive power, which occupies ampacity within transmission and distribution lines. As such, larger conductors and transformers are required to handle the higher currents required to run these types of loads. VArs (Reactive power or Volt-Amps Reactive) represent the resonant energy exchange between capacitive and reactive loads on the grid. Loads that consume high amount of VArs, such as magnetically excited loads like induction motors, power transformers, the inductance in power lines can cause significant voltage drops at start-up. VAr compensation may be needed to boost the voltage back up to acceptable limits. Volt/VAr optimization helps to preserve acceptable voltage levels within transmission and distribution systems in response to changing loading conditions. Volt/VAr regulation also enables some peak shaving, consequently reducing transmission and distribution losses and allowing for a more efficient electricity delivery. 


\subsubsection{Reserves}

As the name suggests, reserves are energy resources that can be activated and connected to the grid at the request of the power system operator. There are two types of reserves, namely, spinning reserves and non-spinning reserves. Up until recently, spinning reserves were resources that were online, synchronized, spinning, ready to produce power or operating below capacity, and upon request, their output power can be increased by the system operator. Although the term spinning suggests that there is a mechanical part that is in motion, with the popularity of ESS, the new definition of spinning now includes energy resources that are not necessarily spinning so long as they can be synchronized to the grid within a few cycles. An example of such would be BESS. In its August 2016 operating manual, NERC updated their definition of spinning reserves as unloaded generation that is synchronized to serve additional demand or load that can be shed [39]. Non-spin reserves are generation capacities that are not connected to the grid but can be called online within a short period of time, usually in less than 10 minutes.

Each of the ancillary services described in this section are very important to the continuous operation of the electric grid because they help prevent disruptions, and they ensure overall grid reliability despite the rising penetration of RER.

\subsubsection{Dispatchable Standby Generation (DSG)}

DSG systems are aggregations of large commercial and industrial generators. Customers that agree to allow utility companies to upgrade, maintain, service and dispatch their on-site 
generators when the need arises are eligible to participate in a DSG program. DSG can be called upon and synchronized with the grid by the system operator within 10 minutes. As such, they provide non-spinning reserve. DSG are dispatched to provide relief to the grid whether the impact is caused directly by RER or not. The problem with DSG is that they only qualify as non-spinning reserves. DSG programs depend on the contracts between the utility and customer, which significantly limit the number of annual run hours. For example, the Portland General Electric (PGE) DSG system has all the necessary functions to monitor and dispatch important power quality data of remotely sited generators. However, PGE is only permitted to dispatch customer generators between 8 and 10 hours annually due to air quality regulations [40]. As a result, most utility companies implementing DSG usually have many participants, though dispatch frequency and duration are low. Although a costly program, the value of the DSG program to utilities is that the assets in the program, although not owned by the utility companies, count towards the utility's non-spinning reserve requirements at a cost that is significantly less than that of dedicated TGR.

The concept and practice of DSG have been researched in literature especially as it relates to outages, brownouts and cascading blackouts [41-44]. For example, Al-Salim et al. presented an agent-based distributed cyclic blackout mitigation and prevention scheme that uses DSG to support customer loads [41]. They demonstrated that it is possible to effectively manage all customer loads by aggregating the power from available DSG resources to power basic loads first, and then after all loads have been sustained and power reduction is required (i.e., during off peak hours), the higher consuming loads are replaced with lower consuming 
loads. Their simulation results proved the effectiveness of this approach in the power loss prevention modes and blackout mitigation modes.

DSGs help address some of the challenges of RER by acting as an alternative energy source that can be used as short term additions to the existing TGR capacity.

\subsubsection{Hybrid Energy Systems (HES)}

HES are a combinations of solar, wind, diesel generators, and sometimes battery energy storage systems. When one source is not available, for example solar energy after sunset, the other resource type can be brought online to continue to serve the load. Coupling HES with BESS is becoming an increasingly more relevant solution. In remote areas, it is not uncommon to have the HES include other forms of energy resources like small diesel generators. HES have been shown to be useful in mitigating some of the problems caused by RER, like their stochastic nature. HES are defined as having at least one RER combined with either a BESS or other types of energy sources like dispatchable generators. HES operate by partly or completely integrating RER in combination with other energy sources, using the strengths of one source to overcome the weakness of the other. Distributed Energy Resources (DER) like PV and wind turbines are combined with generators or BESS to form HES. In remote locations, diesel powered generators may be combined with RER to form HES, and to produce additional power, however, the downside of HES is that they are not very efficient. 


\subsubsection{Demand Side Management (DSM)}

Demand Side Management (DSM) is defined as any means or technology by which a utility can modify its customers' energy consumption pattern in order to suit the utility's need. There are many ways in which participating customers' energy profile and usage pattern can be modified by a utility company. Some of these include, but are not limited to, time-of-use tariffs, Demand Response (DR) programs, load shedding, and load balancing. Often this is done by simply turning on or turning off customer-owned equipment. With proper planning and forecasting, DSM can be used to relieve TGR during peak hours when traditional fossil fuel peaker plants are run for just a few hours (one to four hours) to cover forecasting shortfalls caused by the stochastic nature of RER.

DER can be used to provide DSM. DER include customer-owned assets like air conditioning units, water heaters, commercial pumps, commercial refrigerators, and heat pumps, that can be switched on or off for the purposes of absorbing or shedding power. DER also include small storage assets like batteries with inverters, and electric vehicle chargers, which can be used to both discharge power to the grid or absorb power from the grid.

\subsubsection{Asset Aggregation (AA)}

AA allows for the clustering or grouping of disparate DER to provide grid support services. According to the National Renewable Energy Laboratory (NREL), DER aggregation platforms enable real-time, two-way secure communications and inter-operability between the aggregated assets (or loads), and utility systems [45]. Depending on the context, the term 
'asset aggregation' can be used to mean two different things.

In the first definition, asset aggregation is defined as the use of an application (software or platform) to control a large number of loads. This definition is similar to the one used by Mahmoudi et al., where aggregation is defined as a customer-based (load side) approach to DR. Load shifting, load curtailment, and load recovery were stochastically modelled by the authors through aggregation [46]. In essence, aggregation defined this way is a program or energy platform that can actively participate in the energy market by controlling customer owned loads.

In the second definition, aggregation is a service offered by a third-party entity for the purpose of purchasing power. In this definition, 'an aggregator is a company who acts as inter-mediator between electricity end-users, who provides DERs, and those power system participants who wish to exploit these services [47]. Aggregation, defined this way allows for grouping neighboring electricity customers, small independent power producers or both for the sole purpose of acting as a single entity on the customers' behalf when engaging in the energy markets, either in retail or in bulk [48].

EnerNOC, Comverge, CPower, EnergyConnect, Energy Curtailment Specialist (ECS), Community Choice Aggregators (CCA), and North America Power Partners (NAPP) are examples of aggregation companies that have established individual contracts with customers in the United States. These customers, sometimes referred to in the industry as 'prosumers', have behind-the-meter DERs that can be aggregated and optimized by aggregators and then sold off in the energy market. 
Aggregation is expected to challenge the current operating model of electric utility companies [49]. Due to changes in government oversight and regulations, some consumers in some U.S. states may now choose their electricity supplier, thereby opening retail competition for electricity suppliers and aggregators. The delivery, transmission and distribution of power remains the responsibility of the local power company however. Consumers in deregulated States have the ability to choose or supply their own DER power back into the grid [50].

\subsection{Primary contribution to knowledge}

The primary contribution to knowledge is a solution to the problem caused by RER that uses a novel two-stage method to optimally dispatch the energy take available in the AWHS among frequency regulation, frequency response and peak demand mitigation in a way that maximizes revenue while minimizing over dispatching, system recovery time and energy take forecasting errors.

The energy take of the AWHS is the amount of energy that the water heaters in the AWHS can absorb upon command. The dynamic behavior of the AWHS energy take presents a unique challenge, and in consideration of this unique behavior of the AWHS resource, the developed two-stage optimization algorithm allows the AWHS to maximize revenue, while determining the appropriate ratios to dispatch of each of the three ancillary services. 


\subsection{Motivation}

A market transformation report written by BPA and eight other utilities in the Pacific Northwest in 2018 concluded that if water heaters were to be used as a regional peaking resource, they would only cost $57 \%$ of the total cost of ownership of TGR [5]. In terms of energy, the authors noted that if all resistance electric water heaters in the market as of 2018 are replaced with smart water heaters between 2024 and 2039, based on 2018 loading profile, they will contribute between 340 to $800 \mathrm{MWh}$, depending on the time of day and season, of which $700 \mathrm{MWh}$ of energy can be flexed (shifted from a period of high electricity demand to a period of low demand). This is estimated to provide an economic capacity dispatch value of $\$ 4.9 \mathrm{M}$. The actual costs (purchase, installation, outage costs to upgrade the water heater, and end of life water heater recycling costs) to the utility customers in the Pacific Northwest was reported to be somewhere between $\$ 0$ and $\$ 25$ per kW per year (or between $\$ 0$ and $\$ 112.50$ per year per 4.5 KW water heater) assuming a fifteen year water heater life [5].

A report by the Brattle group found that the benefits of using hot water heaters for ancillary services to participating home owners, after accounting for the water heater costs, could be higher than $\$ 200$ per participant per year [51]. Based on the estimates used in [51] and [5], the annual net benefits to participating customers could be about $\$ 87.50$ per customer.

The BPA report highlights the impact that flexible loads like water heaters can contribute to creating a more reliable grid [5]. Like smart water heaters, there has been a lot of interest 
in batteries, due to their ability to provide fast and accurate responses and bi-directional power. For example, in PJM, formerly Pennslyvania, New Jersey and Maryland ISO (PJM), batteries are used to provide ancillary services like frequency regulation. The deployment of batteries for regulation has increased in capacity from zero in 2005 to over 280 MW by the end of 2017, consisting of 41\% of PJM's regulation capacity procurement [52]. Our research demonstrates that likewise, resistive electric waters can be used to participate in ancillary services markets. 


\section{Literature Review}

\subsection{Literature Review of select current solutions}

Literature reviews were conducted for HES, DSM, and AA, all current solutions used to relieve the grid from the impact of over-generation from RER.

An in-depth literature review of HES is presented in section 2.1.1. The second solution for which there exist an overwhelming amount of research work is DSM. In literature, a lot of DMS research focus on peak demand shifting applications. DSM literature survey is presented in section 2.1.2

The third approach, the focus of this dissertation, is AA. There are many publications in the AA space because many disparate resources can be aggregated to provide relieve to the grid, but findings from our review show that they are different from how the AWHS operates and participates in the ancillary services market. None of this consider the impact that dispatch of DER have on aggregate energy reserve, which is the principle focus of this dissertation. A review summary for AA is examined in presented in section 2.1.3. In 2.1.4 and 2.1.5, we examine the aggregation of $\mathrm{EWH}$, and the aggregation of other assets in literature. 


\subsubsection{Hybrid Energy Systems (HES)}

Bakos et al. demonstrated that the use of different energy sources improves system efficiency and the reliability of the grid, and can help reduce storage requirements when compared to systems comprised of only one RER [53].

Deshmukh et al. presented the different models usually used for the components of most HES systems [54]. In their model, the three performance evaluation factors considered were the loss of load probability, loss of power supply probability, and cost analysis. In their work, the maximum depth of discharge of the battery, temperature correction, rated battery capacity, and battery life were evaluated when modelling the battery systems that accompany the HES. The authors then provided a review of the latest developments and configuration of several HES, including PV-battery, PV-diesel, wind-battery, wind-diesel, PV-wind-battery, and PV-wind-diesel-battery, all of which were presented as commercially viable solutions for addressing the stochastic problems of stand-alone RER.

As a solution to the challenges created by RER, Abdullah et al. discussed how to effectively design and use decentralized power generation, micro-grids, smart grids and stand-alone power systems [55]. While the definition of terms like "micro-grid" and "smartgrid" can vary depending on the application type, the authors conclude that HES can be used for sustainable power system planning in consideration of the economic, environmental, uncertainty issues in power availability of RER. Because at the root of all solar and wind energy resources lies their inherent stochastic nature, the authors recommended coupling RER with BESS, creating a photovoltaic-wind-battery HES. 
Post et al. showed how HES can be used for both on-grid and off-grid applications [56]. Malinowski et al. demonstrated that HES can be used for both off-grid and grid-connected loads with BESS, and a topology for a photovoltaic-wind-battery HES system was developed [57]. The authors discussed the current trends, installation problems, and forecasting issues affecting the global solar market and other PV sectors and their implications on future RER technologies as they relate to HES. According to the authors, an increasing amount of HES are being coupled with solar PV systems.

Yang et al. developed an optimization model for appropriately sizing a hybrid solar-wind HES [58]. A battery bank was used to effectively match the different components of their HES model. Examples of how to model the Levelized Cost of Electricity (LCOE) and Loss of Power Supply Probability (LPSP).

A pilot program carried out in Greece used Monte Carlo simulations to determine the economical benefits of hybrid solar-wind systems [53]. The life cycle costs and payback were determined. A Monte Carlo-based simulation program was used to estimate the energy of the HES. The system life cycle cost was estimated over a 20 year period and a payback period of nine years was determined.

Since the mid 1990's, HES applications have grown rapidly and have demonstrated increasing competitiveness. Up until recently however, HES were used mostly for remote and rural area applications $[59,60]$. Although the cost and technological improvements of HES in recent years have been encouraging, they remain an expensive source of power.

Comprehensive literature reviews of the current and future state of HES using solar 
energy and wind power have been presented by Nema et al. [61] and Zhou et al. [62]. The former discussed the current research status and the future state of wind-solar HES, while the latter addressed the current trends in optimization of HES systems. They both highlight the usefulness of BESS in extending the practical applications of HES.

Further, pairing RER with gas generators reduces the capacity factor of the generator, therefore reducing the return on investment of the HES. As stated earlier, from a sustainability stand point, the environmental ramifications of coupling RER with fossil fuel generators is counterproductive. In addition, optimizing two or three assets in the form of HES presents fewer solutions than optimizing the dispatch of thousands of assets (i.e. like the AWHS proposed for consideration in this dissertation). In addition, HES solutions to the problems of RER is likely to be more expensive, if the controls and integration costs are accounted.

As discussed earlier, that BESS are expensive is one of the first indications that HES cannot sufficiently tackle the challenges posed by RER. Coupling with gas generators is also expensive and counter productive to what RER stands for (i.e., they are renewable).

\subsubsection{Demand Side Management (DSM)}

Applications of DSM in literature are examined in this section. As a note of caution to the reader, the term DSM was found to be interchangeable with DR in literature, although DR is one of the many ways of achieving DSM.

The AWHS has the ability to participate in energy markets either as a DSM application, as a provider of ancillary services or both. Although the literature search revealed that similarities to the AWHS exist in previous works, either for DSM or DER applications, none 
was found to be particularly similar to AWHS in its entirety. In other words, aspects of the AWHS have been implemented by others but none is designed like the AWHS. A limited amount of literature on aggregation of DERs was uncovered in literature, yet only for the purposes of providing DSM.

Gustafson et al. presented a novel method to evaluate the effectiveness of a water heater Direct Load Control (DLC) program for DSM [63]. The DLC programs were implemented by three different utility companies in the Western United States. The findings include methods to apply average customer electricity usage and instantaneous demand to evaluate the potential effectiveness of a direct hot water heater load control program in a given region.

Developed through a comprehensive research program combined with engineering insights into the energy use of house-hold hot water systems, this method resulted in an algorithm for evaluating the potential for load control. Variables monitored through the hot water systems load were presented, and a procedure was developed that allows a dispatch system planner to determine if such a program will be cost-effective compared to a program developed through a more traditional pilot or demonstration approach. This paper also outlined how the same methodology can be used for determining a procedure for the dispatchers to properly initiate and terminate a load control program without hot water recovery problems.

Detroit Edison Electric summarized the effects of field operating conditions on largescale aggregations of electric water heaters in applications for load management systems [64]. Often load control is considered a peak shaving strategy or merely as DSM. Alternatively, 
it can be used by operators to optimize economic dispatch. Findings from this study helped Detroit Edison conclude that load management strategies can be very useful for the refinement of economic dispatch of generation units.

Florida Power and Light has developed a large-scale DLC program for DER customer equipment, which included 50,000 water heaters, 45,000 central air-conditioners, 42,000 central heating systems, 8,000 pool pumps, and 35,000 $\mathrm{kW}$ of commercial space-conditioning equipment [65]. These units were cycled throughout the day based on permission levels determined by customers. While the results obtained were ground breaking, it is worth noting that the underlying goal was to help with load shifting or DSM. The authors concluded that there are three extremely important factors that can help with the integration of DLC programs to the operations of utility bulk power supply. These are customer acceptance, the reliability of hardware, and aggregate load-shaping performance.

Omaha Public Power District and the U.S. Department of Energy conducted several experiments on the application of demand-limiting equipment in all-electric homes [66]. Dual control of demand limiters allowed customers to select the desired peak demand level, which was maintained by the logic of the demand controller. The utility could then reduce the level proportionally by transmission of control signals. Here, no aggregation was applied and this exercise was a DSM approach. Analysis of the results showed that both modes of operation, the local mode and the direct utility control mode, were effective in reducing peak demand.

Wisconsin Electric used a bi-directional power line carrier that enabled its system 
operator to manually control around 92,500 domestic water heater load control receivers at preset times [67]. This was manually done by the system operators, as needed. However, customers were allowed to choose an eight hour window during which their water heaters could not be turned off. The load control receivers send a command to turn on the water heater circuit in fifteen minutes intervals. The water heaters are then turned off for several hours by sending a turn-off command every twelve minutes. This work concluded that DLC strategies using hot water heaters can be used to minimize operating costs by shifting energy usage. Specifically, this reduced the expense of start-up and excessive cycling of TGR that occur when loads begin to ramp up at the beginning of the day.

The energy pattern of about 700,000 water heaters of small residential users were studied by Rautenbach et al. [68]. Users were then grouped based on the switching times of their appliances, water heater size, and house hold size. A multi-objective controller was used to provide a new method of controlling like-kind residential domestic hot water loads with the aims of reducing peak system demand, and minimizing discomfort to the end-user while concurrently reducing their electricity bill. The controller responded to time-differentiated tariffs. Results show that the system peak load was reduced per customer. The authors noted that their control models may not be applicable to commercial, industrial, or other large scale electricity customers.

Ninagawa et al. used Fast Automated Demand Response (FASTADR) to control office building air-conditioning facilities. The authors experimented with 120 different models, each with different stochastic disturbances [69]. Then, a neural network model was built 
using actual buildings facilities' time-series data to test the behaviors and response time of the office building air-conditioning facilities to determine if their step responses were adequate for ancillary services. The authors noted that with FASTADR, some responses received from the controllers tend to oscillate, depending on the communications timing, the signal sampling time, or other stochastic disturbances on the network, and as such, may not be suitable to support ancillary services like frequency response.

Mai and Chung developed a model to control Heating Ventilation and Air Conditioning (HVAC) systems in commercial buildings by using preset time-varying electricity prices to minimize electricity costs [70]. The authors asserted that their research work significantly reduced peak demand and increased energy savings and efficiency, while respecting occupant comfort level.

Of all the DSM literature surveys presented above, none considered the use of DSM for participation in ancillary services markets. Few scholarly publications describe systems that provide ancillary services via DR. Research using DR for ancillary services was published in [71, 72], albeit [71] discusses using DR for frequency regulation alone with [72] being a technical literature review on how to use demand side management of customer loads for the purposes of providing ancillary services like frequency regulation. Rahimi and Ipakchi considered important elements for reliable and economic operation of the transmission system and the wholesale markets, and they reported that under the smart grid paradigm, DR response can be used as a market resource [73].

Other DSM research has been written about extensively in literature [74-77], but they 
focus largely on DR strategies. As stated earlier, DR is a subset of DSM and is the only subset of DSM that was found to use DER as a market resource in literature, suggesting that the use of DSM applications for energy transactions in this way is still in its early stages, and further justifying the need for an aggregator like the AWHS.

\subsubsection{Asset Aggregation}

Several manuscripts discuss aggregation of EWH, batteries, and other assets in an effort to provide a solution to the problem of excess proliferation of RER on the electric grid. An MIT Energy Initiative study from 2013 analyzed utility business models for deployment of DERs including DR, Energy Management Systems (EMS), BESS and PV [78].

However, this study focused largely on the business and policy implications of Asset Aggregation (AA). The MIT study used business operation data from 144 regionallydiverse utilities whose business operations are associated with one or more DER. Technical details of the composition of the aggregated assets were outside the scope of their study. Instead, discussions of the revenue streams, customer segments, and electricity services were presented. Because the utility business models were diverse (144 regions), policy dependent, and heavily regulated, the research concluded that regulation and policy changes need to be considered when developing business models for AA type applications.

Similarly, Zang et al. [79] and Funkhouser et al. [80] both explored the economic benefits of deploying AA applications, but neither particularly addressed the technicalities nor control strategies involved. More so, the aggregated assets were not dispatched to ancillary services markets. Rather, the authors conducted a cost-benefit analysis for the 
customer when involved in certain local utility programs. They studied and analyzed data gathered from utility companies and profiled the type of services, based on the business model and customer outreach programs at the respective service territories.

Koliou et al. used DR to aggregate customer loads for DSM [81]. They investigated how DSM of aggregated loads can be used as a resource for balancing the grid. Small customer loads were bundled and aggregated for transactions in the German energy market. The authors illustrated how aggregation companies can bundle up small customer loads and use this as a market participation resource. The authors noted however that as a viable market resource, aggregation of DR loads for DSM is still in its early stages of consideration. Pruggler came to similar conclusions, demonstrating the economic potentials of DSM, especially for spot market-oriented loads at house hold levels [82].

Calvillo et al. noted that the market share of aggregators in the energy market globally was between $1 \%$ to $2 \%$ in 2016 , and that proper planning and efficient operational strategies, along with friendly government regulations, will likely help grow the role of aggregators [83].

DERs that provide DR were referred to as flexible service providers by Eid et al. [84]. In their work, the authors argued that many barriers still exist that limit aggregators from participating in balancing markets and that policy makers and regulatory commissions should assist to lower those barriers and develop better compensation mechanisms between aggregators, utility companies, and generation suppliers. They concluded that such flexibility services are necessary for the reliability and sustainability of the grid. 
A review of other literature discussing AA and select pilot-projects was presented by Neisten and Alkamade [85]. In their analysis, they reviewed the data from 434 European and US smart grid projects. They noted that an aggregator is critical in making any market participation of customer DERs in the energy market economically viable. The authors further demonstrated that aggregation of assets (EV batteries in this case) and the role of aggregators is necessary to further the business case for the wide adoption of a variety of other smart grid services for market participation.

Motalleb et al. demonstrated how distributed DR scheduling can be used to provide frequency regulation during contingency periods [86]. DERs like battery banks and electric water heaters were used in aggregate as sources of ancillary services. The researchers implemented a control system model and specialized algorithms that optimized the DERs for DR. Two points worth noting are that this paper focused on DR alone, and secondly, the algorithms presented were limited to when contingency events occurred on the grid.

Roos et al. developed an optimization framework for a load aggregator participating in wholesale power and capacity regulation markets. They used actual data from a set of Norwegian electricity consumers to test the model and estimate the value of aggregation to the market [87]. This report concluded that the aggregator value largely depends on factors such as daily price variations, the definition of market on-peak and off-peak periods, and the price of storage, among other factors. The aggregator's objective was to minimize the total energy costs to the consumers. Select customers for this study included shopping centers and food production sites with loads such as heating, air conditioning, and lighting. 
The technology to do this was developed by Enfo Consulting AS, a European smart grid solutions company that enables communications and control for residential loads.

CAISO conducted a pilot study in collaboration with Lawrence Berkely National Lab (LBNL) in 2009 that determined the feasibility of allowing commercial and industrial DR-enabled DERs to participate in wholesale day-ahead non-spinning reserve ancillary service markets [88]. The objective was to assess the technical and financial feasibility of using retail loads to participate in day-ahead wholesale non-spinning reserve ancillary service markets. Three facilities, a retail store, a local government office building, and a bakery were used as DER resources and linked together using Open Automatic Demand Response (OPENADR). OPENADR is a public (non-proprietary) standardized interface that allows electricity providers to communicate DR signals directly to existing customers using a common protocol. With OPENADR, communications can be easily exchanged between many devices through a unified messaging system. Energy pricing and reliability signals can be readily exchanged via OPENADR to utilities, independent power operators and system operators. The results showed that assets controlled using OPENADR could be used for some types of ancillary service markets. The CAISO and LBNL researchers also found that DR strategies for HVAC and lighting can provide responses suitable for participation in the non-spinning reserve ancillary services market. This research focused on optimizing the communication and telemetry infrastructure needs, understanding the capabilities in commercial and industrial facilities to automatically deliver load within the limitations of the non-spinning reserves product, and testing the feedback controls to maintain the 
commitment of loads.

An aggregator was proposed for time-of-use applications by Rahnama et al. [89]. A supermarket refrigeration system and a chiller with ice storage were used in a case study. Results obtained from the study were verified against actual supermarket energy use to determine potential profitability. A centralized controller, whose responsibility is to aggregate load flexibility in an optimal way based on preset market time-of-use prices, was used for large commercial customers.

Ruiz et al. created a Virtual Power Plant (VPP) by aggregating several DER loads (air conditioning units, water heaters, electric space heaters) [90]. This was accomplished by aggregating the capacity of the DERs in order to make them more accessible and manageable in the day-to-day energy markets. An algorithm to manage the VPP consisted of a large number of customers with thermostatically controlled appliances. The algorithm, based on Direct Load Control (DLC), similar to Diduch et al. [91], determines the optimal control schedules that an aggregator should apply to the controllable devices of the VPP in order to optimize load reduction over a predetermined duration. The results define the load reduction bid that the aggregator can present in the electricity market, but the model used for the bids are not flexible nor are they divisible. They were constrained by the parameters of the models, which need to be reset every time a new customer is added.

The possibility of providing regulation services with small loads, such as water heaters, electric heaters, or air conditioners was researched by Kondoh et al. [92]. Specifically, DLC models were developed for aggregating water heater loads for the energy market. The 
models estimate the minimum amount of water heaters needed, the duration of regulation, as well as the amount of regulation (MWh) needed. The researchers concluded that the aggregated regulation service provided by water heater loads can become a major source of revenue for load-serving entities. To guarantee customer comfort and provide regulation service, however, the water heater thermostat control circuit was modified. Because each thermostat control circuit was modified, scaling could be a challenge. One limitation of this work is that from 00:00 hours to 06:00 hours, regulation is not available due to lower power consumption of the water heaters.

A method was developed by Keep et al. that allows for the use of aggregated electric loads to balance forecast shortfalls on the ACE [93]. The strategy is quite unique compared to other literature surveyed thus far in that it relies only on load switches as the source of local control actuation, yet it is capable of both decreasing and increasing the total aggregate load while causing little to no disruption to the end users. Load switches were used for controlling refrigerators. However, the application of aggregation to household refrigerators alone limits the scope of this work. The authors presented a mathematical model for the control algorithm, and they noted that other appliances like space heaters and water heaters should be expected to provide similar results.

In summary, although the definition of aggregators varied widely between the different literature examined, they were all consistent in their need for providing support to the electric grid. Further, how the aggregated services were bundled was also different between authors. The definition of 'assets' is particularly important because some authors describe assets as 
RER that produce power to the grid (one way output, i.e., PV) while others describe assets as customer loads that have the ability to load up or shed energy (bi-directional). As Burger et al. noted, the interpretation of aggregators can be expanded or restricted depending on the roles and functions of the aggregators [78]. Most literature refers to AA in the first context, suggesting that the later (bi-directional generation and/or absorption) is not yet mature, albeit, both types of aggregation hold significant benefits to solving the problems created by the ongoing proliferation of RER on the electrical grid.

\subsubsection{Aggregation of EWH}

Laurent and Malhame built a computer aggregation model to simulate aggregations of water heaters [94]. They tested the influence of certain factors on power ratings including insulation, element power rating, and variance in water demand. Their work was targeted for modeling the behavior of water heater aggregations for forecasting loads, not controlling or dispatching them for market participation. In another work, Laurent et al. created an optimization method for load management with control of electric water heaters [95]. They used a column generation method to optimize the water heaters for peak load reduction.

A Direct Load Control (DLC) algorithm was developed for aggregated control of domestic electric water heaters by Diduch et al. [91]. Some of the challenges encountered in this experiment were due to uncertainties in estimates of the water heater temperature fluctuations, water extraction, and demand estimate, and forecast of reserve load. The authors recommend using load modeling as a means of improving the accuracy of the algorithms developed. In their aggregation of water heaters, the authors demonstrated that 
water heaters can be used as an energy storage buffer when peak-shifting power scheduling schemes are in force.

Fitzgerald et al. modeled 100,000 EWH assets to improve efficiency of wind generation [96]. They designed a control algorithm to improve the likelihood of water heaters being able to take on extra load during an expected peak in wind generation. Their simulation showed that applying their algorithm could result in electricity reduction of $25 \%$ and cost reductions of $38 \%$ from scheduling power consumption at better times.

Kepplinger et al. tested demand side management control optimization schemes for aggregated EWHs [97]. They tested three optimization methods: a price driven optimization, an energy driven optimization, and an optimization method based on time of day. They determined that a price optimized control scheme was most cost efficient with fewer service errors.

Kapsalis and Hadellis created an optimal scheduling algorithm for EWHs [98]. They used an objective function that weighed interests in keeping electrical costs low while keeping customer discomfort minimal. Their algorithm minimized both costs and discomfort based on relative weightings. Their algorithm worked on two levels, being able to adjust customer set-points and having access to a direct on/off switch for the electrical coil. In a related work, Kapsalis et al., presented a heuristic scheduling algorithm to balance decisions between cost of power and comfort of consumer hot water consumption [99]. The authors simulated their algorithm in a real-time market and found it performed as well as a standard optimization model with considerably less computational overhead. 
Roux et al. developed a peak demand manager algorithm that focused on water heater usage. Essentially, they had EWHs compete to use the electrical grid during peak hours, and only water heaters with the most need turned on during peak hours. They tested their control method against a traditional control method, and found their algorithm could shift controlled amounts of electricity from peak times, with minimal events of user discomfort [100].

\subsubsection{Aggregation of Other Assets}

Li et al. created an aggregator service for large multi-tenant buildings [101]. They built an aggregation of EWHs, solar panels, battery energy storage, and electric vehicles. They examined control algorithms for the DER assets that would allow them to minimize power costs through load shifting and energy trading of the DER units.

Faika et al. proposed an Internet of Things (IoT) architecture for aggregating multiple batteries over a wide area network [102]. They tested their proposal on a five battery test bed and found the latencies in the network were small enough to allow for massive scaling over a cloud network.

Khalid and Savkin analyzed the aggregation of batteries to smooth wind power production [103]. They used the aggregation to compensate for small dips and rises in wind power. They found a semi-distributed system of batteries both near the wind turbines and away from the wind turbines was most effective at smoothing wind power.

In the field of aggregations using DER, Asimakopoulou and Hatziargyriou modeled the economics of DER aggregation through a bilevel programming problem using a DER agregator's bid decisions as the upper level problem and the market clearing decisions as the 
lower level problem [104]. They confirmed that the addition of DER assets had a stabilizing effect on locational marginal prices (LMPs) in the bulk electricity market. They found that when LMPs were high, DER aggregators performed best when exporting energy to the power grid, and when LMPs were low, DER aggregators performed best when importing energy from the power grid.

Calvillo et al. built an optimization model for a variety of DER assets including photovoltaic systems, air-sourced heat pump systems, batteries, and demand response assets [105]. They optimized a system using price-maker economics, assuming that the DER aggregation was large enough to influence market clearing prices. They analyzed aggregations of residential houses running from forty thousand to eight million houses. Calvillo et al. found that aggregating these assets could be profitable for both aggregators and prosumers.

We reviewed a few papers that discussed battery aggregations using Electric Vehicles (EVs) in order to gain an insight into how much work has been done in this area. Wu et al. determined the operation scheduling of an aggregated set of EVs from a cost minimization perspectives. Ortega et al. discussed the changes necessary to allow for aggregated EV dispatch in a day-ahead electricity market. Gonzalez and Andersson, Vagropoulos and Bakirtzis, and Sarker et al. all investigated optimal bidding strategies of EV aggregations [106-108]. 


\subsection{Literature Survey of Select Optimization Techniques}

With thousands of water heater energy profiles to to be aggregated by the AWHS, it is critical that current optimization techniques discussed in literature be explored. In the following paragraphs, several optimization techniques pertaining to DSM, HES, AA, and RER are discussed. Optimization techniques were explored in literature because it can help inform how the AWHS can better dispatch its resources to maximize revenues.

\subsubsection{Particle Swarm Optimization}

Maleki et al. used a Particle Swarm Optimization (PSO) algorithm and Monte Carlo methods to optimize HES systems [109]. PSO is an heuristic searching technique that uses stochastic optimization on a population that is initialized with a population of random solutions. Then, an algorithm is used to search for the best results by updating the outputs. The authors used Monte Carlo simulations to randomly generate stochastic demand and supply profiles, including load profiles at households, residential solar generation profiles, and micro wind-turbine generation profiles. A battery was used to store the generated energy.

Zhou and Sun proposed a type of PSO algorithm for HES, referred to as "simulated annealing" PSO [110]. The new algorithm improves the diversity of PSO and improves the ability to finding new optimum results. The authors concluded that the global searching ability of this new method was improved. By optimizing the capacity of HES systems, the authors found that the upfront economic investment, and the operation costs can be significantly lowered on the basis of maximizing the renewable energy output and meeting 
variable demand loads.

PSO has been used with stand-alone RERs like solar and wind. Saad et al. used PSO to optimize the maximum power point tracking capability of grid-connected solar PV systems. Results presented by authors showed that PSO helped reduce excessive DC link voltage, high AC currents and loss of grid-voltage synchronization [111].

Kayal and Chanda used a simple but efficient PSO approach for optimizing the siting of solar PV panels and wind turbines within an electrical distribution territory. Factors considered included electrical network power loss minimization, voltage stability and network security requirements [112]. Weighted aggregation PSO was used to optimize the RERs considering bus voltage limitations, maximum line loading capacity, capacity limitations and penetration constraints of the RERs. Strategic weight selection techniques were then applied to evaluate performance trade-offs for each type of RER relative to its placement within the distribution network placement.

Chang demonstrated how PSO can be use to find the Weibull parameters of three wind turbines located at different climates [113]. The Weibull distribution is an expression that gives a good fit for the total mean power generated by a wind turbine relative to a range of mean wind speeds. It provides a probalistic base model that can be used to optimize wind energy harvesting. The authors concluded that PSO is feasible for wind energy applications because of its rapid convergence in estimating Weibull parameters, regardless of the data size or differences in wind patterns from one turbine to another.

Aghajani et al. proposed a multi-objective energy management system for the perfo- 
mance optimization of RERs [114]. The problems with operating RERs were then solved considering the responsive loads as coverage for the uncertainties associated with wind and solar power generation as an optimization function. Considering the complexity and non-linearity of RERs, Multi-Objective Particle Swarm Optimization (MOPSO) was utilized. Fuzzy-based mechanisms and non-linear sorting of the overall system were then applied to determine the best compromise considering the set of solutions from Pareto-front space. The numerical results obtained from the research represented the effect of the proposed DSM scheduling model on reducing the uncertainty obtained from RERs. Albeit, their work discussed using DSM without the specifics of the types of DSM and limited consideration was given to aggregation of DERs.

\subsubsection{Hybrid Big Bang-Big Crunch}

Hybrid Big Bang-Big Crunch (HBB-BC) is a non-linear evolutionary computer algorithm that can be used to solve large complex optimization problems with larger constraints, time and memory requirements [115]. Some optimization problems can be solved using linear programming and quadratic equations, but these tend to have reduced accuracy $[116,117]$.

For more complex algorithms and non-linear optimization problems requiring a lot of iterations before convergence can be achieved, HBB-BC can be used. Ahmadi and Abdi used a HBB-BC algorithm for optimal sizing of a stand-alone hybrid PV/wind/battery systems [118]. They compared the performance of the HBB-BC algorithm to that of PSO and then later, to that of a Discrete Harmony Search (DHS) algorithm. For applications with HES, they found that the HBB-BC algorithm had the highest accuracy, best performance and 
could find the most optimal solution when compared with the other optimization techniques examined in their study.

\subsubsection{Time Series Regression Analysis}

Time series regression analysis is the application of statistical methods to existing historical data for the purposes of predicting future outcomes. For example, utilities can use prior customers energy usage patterns over a prolonged period of time (at least one year) for planning and system wide growth forecasts. Time series regression can help with forecasting of economic, financial or energy usage behaviors.

Average hourly whole-house load data from 195 participant households and 268 nonparticipant households were compared after the participant thermostats were remotely controlled for four hours on five event days in a study conducted by Newsham et al. [119]. Complex time series regression analysis was applied to the two datasets. The authors reported $10 \%$ to $35 \%$ average peak load reductions per participant household when compared with the non-participant households.

\subsubsection{Other Optimization Techniques}

Aslam et al. proposed an effective DSM management program developed using metaheuristic Genetic Algorithm (GA), Cuckoo Search Optimization Algorithm (CSOA), and the Crow Search Algorithm (CSA), for electricity cost and peak load alleviation with the least amount of customer waiting times with the integration of smart energy storage systems [120]. Thirty smart homes, and real-time energy pricing and Critical Peak Pricing (CPP) 
signals were examined in terms of electricity cost estimation for both a single smart home and a smart building. The simpler but equally optimal Approximate Dynamic Programming (ADP) was used by Al-jabery et al. for energy management of domestic EWH [121].

Elhadidy and Shaahid, and Beyer and Langer used Multi-Objective Evolutionary Algorithms (MOEA) to solve the problems with HES like system costs, system reliability, design optimization and control $[122,123]$. Augustin and Dufo-Lopez published a literature review paper that covers the types of simulation and optimization techniques, as well as the tools existing used to design stand-alone HES [124]. Design and optimization software tools like HOMER, HYBRID2, HOGA and HYDROGEMS were discussed in their review.

\subsubsection{Related Optimization Work}

Due to the ongoing large scale integration of RER and the challenges they pose, there is a need to optimize their operation in tandem with other distributed assets i.e., battery storage systems, customer loads, so as to maximise their benefits to the grid-at-large, enhance system wide reliability, and make a return back to rate payers or private investors. Some of the energy optimization schemes uncovered in the literature includes Brunelli and Tamburini's work on developing residential load scheduling optimization schemes for energy cost minimization. However, their work was limited to a single hybrid system comprised of electric household appliances in tandem with PV and storage at a specific location (i.e., one household system alone) [125]. In the work by Mangiatordi et al., an optimization scheduler was developed for multiple households, but the energy price control was not factored in their optimization [126]. Likewise, kardaras et al. developed a way to optimize demand response. However, 
their system did not include PV or storage [127]. An optimization system based on a charge/discharge scheduling algorithm for battery storage systems operating in tandem with PV was developed by Babacan et al. Their system helped minimize the monthly electricity expenses of a customers by incorporating both a time-of-use volumetric tariff and a demand charge tariff [128].

Others have performed scheduling for appliances, but they do not account for aggregation. By far, the most comprehensive is the work by Passenberg et al. [129]. They developed an optimal water heater control using a smart control system. Similar work has been performed at the power lab at Portland State University in the research work by Emily Barrett [130]. In this case, a hot water heater was optimized. In Barrett's work, differential evolution and alpha prototype strategies were optimized in a two-stage heat pump system, which was shown to achieve a $14 \%$ reduction (and about $20+\%$ improvement during winter months when price-based programs are most capable of performing peak-shaving) in the wholesale electricity cost of operating a heating system with some storage (thermal storage) in a generic single family residence. Also from Portland State University was Robert Rector's work on Generic Differential Calculus Optimization. Rector demonstrated how smart solar inverters and battery storage systems can be used to maximize the revenue earned for providing real and reactive power sales [131]. 


\subsection{Dispatch of Residential Water Heaters for Ancillary Service}

Because of their fast response time, water heaters can participate in more than peak demand mitigation. There are several utility programs where water heaters are used for peak demand mitigation alone $[65-67,132]$. To date, not many have tried to use hot water resources for more than one ancillary service simultaneously. The financial gains in doing this, as summarized by Hledik et al., is that a service that allows the use of water heaters for frequency response can bring more benefits [51]. Hledik et al. demonstrated this both with the PJM ISO and the Mid-Atlantic Independent System Operator (MISO) markets.

The reason why water heaters have not been used on a large scale to provide ancillary services is due to the the unwillingness of water heater manufacturers to open up their proprietary platforms to allow control access by non-Original Equipment Manufacturer (OEM) vendors. BPA's recent market transformation report showed that the costs of retrofitting water heaters with communications and control modules that can allow a communication protocol like CTA 2045 or OPENADR are negligible if rolled out and programmatically implemented at utility-level scale [5].

Recently, there has been concrete efforts to standardize remote access control of water heaters. The State of Washington in 2019 passed a bill that will mandate all new water heaters sold within the state to be CTA 2045 compliant beginning in $2022 .{ }^{10,11}$ When enacted, this will establish a precedent for allowing control of customer owned water heaters

\footnotetext{
${ }^{10}$ Washington State Legislature, http://lawfilesext.leg.wa.gov/biennium/2019-20/Pdf/Bills/House20Bills/1444-S.pdf

${ }^{11}$ Washington State Department of Commerce,https://www.commerce.wa.gov/growing-theeconomy/energy/appliances/
} 
by $3^{\text {rd }}$-parties outside of water heaters OEMs' proprietary platforms. This should encourage more willingness from both customers and utilities to exploit the capabilities of water heaters for providing utility services. 


\section{Methodology}

\subsection{Introduction}

We propose a two stage algorithm for optimizing the available energy in the AWHS by using market price signals to maximize revenue. In stage 1 , we begin by simulating and initially optimizing all the units available in the AWHS as if they were all reserve resources. This initial optimization relies on forecasts. The optimization routine anticipates future energy take, weather temperature, and market prices based on historical data. Upon convergence, the optimization algorithm shifts to the next time block and re-iterates the optimization with new projected allocations. This process repeats continuously until a full day's worth of projections are simulated and optimized.

In stage 2, the AWHS considers how dispatches affect the AHWS resource. It reoptimizes to maximize revenue for each ancillary service based on the effects that dispatches have on the AWHS energy take resource. Over-dispatching is prevented by limiting dispatch capacity of the ancillary services. If dispatches were not limited, the AWHS could run low on energy take, which could limit its ability to earn revenue.

A summary of the method is presented in Figure 3.1. 


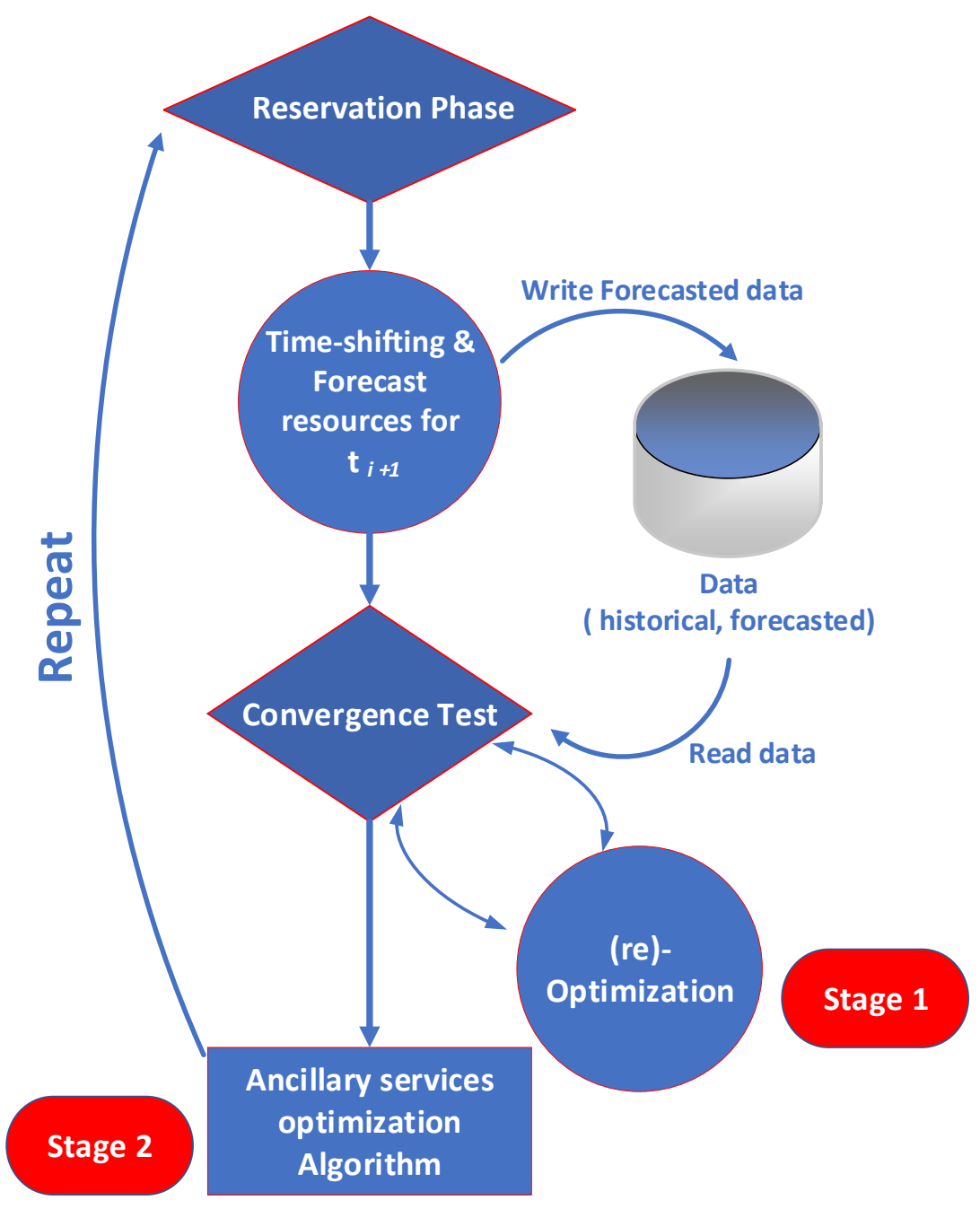

Figure 3.1: Optimization Method Summary. 


\subsection{Justification for the proposed methodology used in this research}

Of the six solutions described in section 1.8 for addressing the challenges of RER, AA using residential electric water heaters was chosen. We chose to aggregate water heaters because of their quick response time, the ease of scaling water heaters by the thousands in our models, and because water heaters are ubiquitous. Electric water heaters are in more than 50 million homes across the U.S, and they account for more than nine percent of all electricity consumed by households nationally, with more than forty percent of U.S households consisting of at least one electric water heater [51]. A number of sources have demonstrated that customer loads can provide superior response in comparison to generators, because the curtailment of load typically responds faster to control signals than larger and more complex TGR [133-138]. The following subsections describe the steps involved in the optimization method summary in Figure 3.1.

\subsubsection{Stage 1 and Stage 2 Optimization}

The optimization begins with the reservation phase in stage 1 . This is because all services are treated as reserve services at the beginning of the optimization. The reservation phase advises the optimizer of when to schedule services, and how much to schedule.

By considering all services to be reserve services, the first stage optimization produces a service schedule that does not impact the AWHS energy take. This schedule advises the stage 2 optimization phase of when dispatch services may be optimally scheduled. In stage 2, the impact of dispatches on the AWHS energy take profile are considered. 


\subsubsection{Time shifting}

This research is novel in that we attempt to allocate a regenerating resource over multiple time periods with two major limiting factors, namely, uncertainty and impact. There are uncertainties present due to forecasting of future energy take, weather, and price data. The impact is that every dispatch affects future dispatches due to the system recovery time. Such an impact is not an issue at gas plants, for example, because there are reserve supplies of gas with a steady supply to the generator, and barring any sudden or unplanned outages, the generator control room operators know precisely how much energy capacity it can provide at any time. To compensate for this impact, we optimize the resources available in the AWHS in 5 minute step-intervals. We keep looking into the future so we can compensate for the effects of over-dispatching, which can result in prolonged recovery times for the AWHS. Prolonged recovery times can result in a high opportunity cost for the AWHS, as energy take becomes unavailable for scheduling to reserve services.

The work done here addresses a challenge with dispatching a dynamic resource like the AWHS, namely, how the system recovery can be managed in a way that adequately positions the AWHS to participate optimally in the next round of bidding following the last dispatch at $t$-1. The resource recovery curve represents the energy take left in the AWHS immediately following a dispatch, and it shows the duration that it will take the AWHS to get back to its pre-dispatch energy-take level. The resource recovery curve is recalculated after each dispatch. Further, the energy dispatch constraints are dispatch-dependent and need to be recalculated for every selection of dispatch vectors. 
This method slides the entire 24 hour daily window (288 five minute time intervals) ahead by 1 unit of time ( 5 minutes), i.e., from 1 to 288 to 2 to 289 , etc. The outcome is that the optimization schedule changes as new future data projections are added. After every dispatch, the available energy take decreases and so a new energy curve is redrawn.

Following each dispatch, and in preparation for the next dispatch, our algorithm timeshifts (slides ahead) to the next five-minute interval based on historical data. By time shifting, we are able to optimize for future dispatches, in anticipation of the changing energy take profile of the AWHS. In the following paragraphs, we explain how we used time series forecasting to inform our two stage optimization algorithm.

\subsubsection{Time Series Forecasting}

Forecasting of time series data is not new. It has found several applications in both industry and academia. In an effort to improve accuracy, several time series forecasting methods and models have been postulated for a wide variety of applications. A survey of different time series forecasting methods were presented by Mahalakshmi et. al [139]. The authors discussed time series methods for forecasting electricity market clearing price and electricity load forecasting data. Similarly, for our research, we elected to forecast future energy take time series data, and future energy prices time series data. We selected Long Short Term Memory (LSTM), a form of Recursive Neural Networks (RNN), for forecasting in this dissertation. 


\subsubsection{RNNs and LSTM}

RNNs operate by processing inputs, and then predicting $n+1$ output. RNNs were used for classification initially but now they are used for time series prediction. The problem with RNNs is that when the hidden layers are fed back in, they can be very difficult to train right, can be very difficult to use, and sometimes are not good at predicting long time series data sets. LSTMs are an improvement to RNNs because of the way RNNs work. With RRNs, older information has to travel through several steps before getting to the present processing step, increasing the possibility of getting easily corrupted by being multiplied several times by many small numbers. This problem is known as the vanishing gradient problem. In simpler terms, the vanishing gradient problem is caused when using RNN for time series forecasting because the output at a particular time depends on the input and the outputs of previous steps. Because the output will change every time the input changes, for long data sequences, the gradient gets smaller and smaller and is said to vanish. This quickly becomes inefficient for longer data sets since the output changes every time there is a new input, and with a longer duration of time. ${ }^{12,13}$ LSTM on the other hand makes decisions by considering current input and previous output, and it has an extra cell, a memory cell, it then generates new output data and alters its memory [140]. LSTM is the optimal solution for our forecasting needs because we have a large data set and we need to forecast over long periods of time.

There are many scholarly works referencing the use of LSTM for time series forecasting.

\footnotetext{
${ }^{12} \mathrm{https}: / / \mathrm{mc}$. ai/how-do-lstm-networks-solve-the-problem-of-vanishing-gradients/

${ }^{13} \mathrm{https}: / / \mathrm{www}$.superdatascience.com/blogs/recurrent-neural-networks-rnn-the-vanishing-gradientproblem
} 
However, due to similarity of their work to the topic of this dissertation, we reviewed the works of Panapongpakorn et al [141], and De et al. [142]. Panapongpakorn et al. used Long Short Term Mermory (LSTM), to propose 30 minutes load forecast based on time series analysis and neural network methods in an Energy Management System . The performance of their models was evaluated using Root Mean Square Error (RMSE) and mean absolute percent errors. De et al. on the other hand forecasted photovoltaic power as a means for anticipating and predicting shortages. Due to the stochastic nature of photovoltaic, the authors developed a model for accurately forecasting photovoltaic power with high accuracy by using LSTM.

\subsubsection{Justification for using LSTM}

LSTM is a type of RNN that can learn the order and sequence dependence of data in events predictions and forecasting. ${ }^{14}$ LSTM is commonly used today in applications like language modeling, speech recognition, and machine translation. There are several benefits of LSTM over other time series forecasting methods like single or multiple regression methods, exponential smoothing, single layered Support Vector Machines (SVM) or other RNN methods like Gated Recurrent Unit (GRU). Perhaps the most advantageous is that LSTM solves the vanishing gradient problem that occurs, particularly for predictions using RNN, when there are long sequence time series data involved. According to Chung et al., most RNN solutions have a recurrent layer that provides memory for a given amount of

\footnotetext{
${ }^{14}$ Machine Learning Mastery, https://machinelearningmastery.com/gentle-introduction-long-short-termmemory-networks-experts
} 
time. However, over longer time periods, the gradient of the memory decay causes reduced information retention [143].

\subsubsection{Forecasting using LSTM}

The time period when forecasting occurs is for $t_{i+1}$. Time shifting occurs before forecasting is applied, and the entire dataset is shifted by one 5 minute time interval. This is done so as to prepare, optimally, for the next dispatch. In our research, we apply LSTM on the time shifted data to help inform future resource availability based on the following; past dispatch data, a user pre-defined learning rate, number of iterations, and the root mean square errors in between iterations to inform future projections. The obtained projections are then stored in memory for future retrieval based upon the next dispatch time.

\subsubsection{Testing and training the data}

We use MATLAB's LSTM functions for training and testing of our data sets. The following process flow diagram shows the step by step approach used for the testing and training process. 


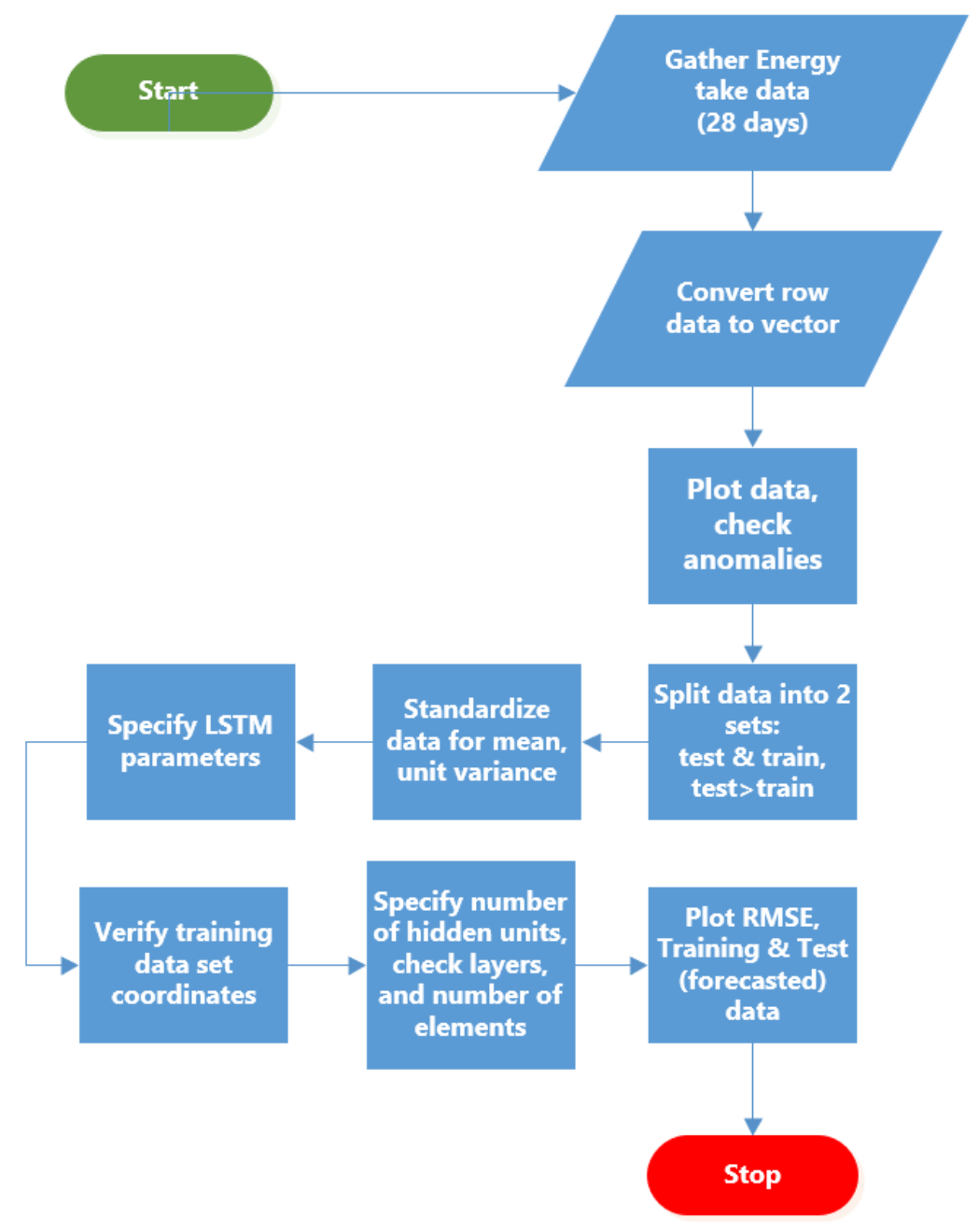

Figure 3.2: LSTM test and training flow diagram.

\subsubsection{Accuracy of forecast results and convergence test}

The level of accuracy expected of the LSTM used in this work was chosen arbitrarily as 5\%.

Most utilities, depending on their expected revenue targets will have internal accuracy targets.

These targets are expected to vary between utilities. We define convergence as when the forecasted data are within five percent of historical data. Because we have historical energy take data, we know what past AWHS load profiles look like, and we also have historical 
market price information from PJM. By applying our convergence test, a decision is made if the forecasted data should be passed to the next phase. This is done by continuously comparing the time shifted forecasted data from the previous latest forecast to the available historical data. Forecast data that fail the convergence test are passed through additional rounds of forecasting until convergence is achieved. Similar to the work of Panapongpakorn in [141], the RMSE in our results were both found to have a less than $2 \%$ error as shown in Figures 5.21 and 5.24 for energy take and LMP pricing respectively. Therefore, with LSTM, we were able to exceed our 5\% prediction accuracy target.

\subsection{Proposed Solution to the AWHS Optimization Challenge}

Of the three services under consideration, frequency response and peak demand are reserve services while frequency regulation is a dispatch service. A dispatch service turns the AWHS resource on at a scheduled time to consume specific amount of energy. A reserve service withholds load from being dispatched. A summary of these services is presented in Table 3.1.

\begin{tabular}{|l|l|l|}
\hline Ancillary Service & Market type & Dispatch requirement \\
\hline Peak demand mitigation & reserve market & never dispatched \\
\hline Frequency regulation & dispatch market & is dispatched whenever scheduled \\
\hline Frequency response & reserve market & rare, stochastic dispatches \\
\hline
\end{tabular}

Table 3.1: Classification of the three services under consideration.

In the first stage of the optimisation algorithm, we optimized the AWHS as if the entire resource is available by treating it as a fixed resource for the first iteration and then solving the entire energy dispatch within the 24 hour window. This is the same as if there were no 
energy dispatches during this time period. The number of units available if we are to do no dispatches is not going to be the same if we were to dispatch the AWHS. The challenge is how to calculate the energy take available after a dispatch. In the second stage, a dispatch will change the AWHS energy take and the number of units available for future scheduling. The available energy take will change throughout the day as the number of units available and the amount of energy take available changes, requiring recurring resource evaluation as time advances.

In summary, we solve this challenge in two stages. In the first stage, we optimize a dispatch schedule for the AWHS assuming all three services are reserve services. In the second stage, we accommodate the impacts of the dispatch service. We execute the first scheduled dispatch at time $t_{x}$, re-draw the energy take curve of the AWHS, and then re-optimize for $t_{x+1}$ for $t_{x+1}<t<288$. This is repeated until the last scheduled dispatch within the window is evaluated.

\subsection{Rational for the ancillary services under consideration}

Due to the inherent nature of resistive water heaters, they are able to respond very quickly to control signals. This feature of water heaters makes them well suited for some ancillary services. Two of the services chosen in this work, namely, frequency regulation, and frequency response both require quick response from the AWHS and were chosen for this reason. Peak demand reservation, on the other hand, is an ancillary service that requires a large amount of energy consumption to be time-shifted. Water heaters are suitable for peak 
demand mitigation and were chosen for this work because hot water usage can be decoupled from energy consumption, and because water heaters store significant amount of energy. The following paragraphs detail why the other three services, namely, ramp rate control, Volt/VAr, and reserves were not chosen for this work.

Ramp rate control is the ability of an energy resource to change its output over a specified time interval. By increasing or decreasing the energy output of a generator, the generator is said to be ramping up or ramping down, respectively. A generator ramp up is equivalent to a load ramp down, and vise versa. Because of how quickly the water heaters can turn on, they can perform very well for decrement ramping events. In other words, if the system needs generation to ramp down, the AWHS can alternatively ramp water heater loads up to achieve the same objective. However, in order for ramp rate control to be included in the optimization, it would need to be considered as a constraint of the optimization. Since the ramp rate requirement vary throughout the day, based on the needs of the grid, such a constraint would need to be modified constantly. In practise, the nature and the complexity involved in dynamically modifying the ramp rate of the AWHS is why we have opted to exclude this otherwise important service from our work. However, water heaters have very high ramp rates, so they would likely be suitable for meeting most all decrementing ramp requests.

Secondly, the Water heaters considered in this research cannot be used for Volt/VAr because they are purely resistive in nature with no capacitance or inductance. Volt/VAr control is achieved through addition of capacitive or inductive elements to the grid, or 
through the use of power electronics to adjust the phase angles of an inverter to either lead or lag the voltage at the point of interconnection. Water heaters do not have these capabilities.

Thirdly, in terms of system reserves, reserves are resources that can be called upon when needed to respond to stochastic events. Frequency response, also stochastic in nature, has been modelled in our work. We found no additional benefit of studying another stochastic event. More so, in some markets, non-spinning reserves are not compensated. Because the AWHS is a non-spinning reserve, we found only little benefit in modelling the AWHS as a reserve if it is not universally compensated for [144]. Revenue maximization, the objective of this work, can not be achieved for non-compensated services.

\subsection{Difference between this work and other existing utility programs}

Although, many utilities have aggregation platforms and programs that aggregate water heater resources, the body of work presented here is different from such platforms and programs in the following ways:

- Many utility programs are limited to demand response only. They do not address frequency regulation or frequency response fluctuations.

- Utility programs only participate in energy conservation strategies by disabling water heaters temporarily. They do not participate in active energy take dispatches.

- In most cases, such programs are not automatic but rely on a program administrator to send out a mass communication campaign to subscribed customers who may choose to either opt in or opt out of participation at the time of the event. Our solution 
here however relies directly on market price signals with no middle-man or human oversight-scheduler/program manager involvement.

- Utility programs do not account for impacts caused by the dynamic nature and changing energy take in the AWHS, and

- Most importantly, utility programs do not perform optimization like our proposed two-stage method. 


\section{Description of Method}

For our research, we modelled and simulated an AWHS comprised of 10,000 residential EWH. The water draw profiles of the water heaters in the AWHS are defined using the water draw scheduler developed by NREL [145] that develops water draw profiles based on household size information gathered from U.S. Census data [146]. The data encompass 12 months in 288 five-minutes daily intervals.

\subsection{Optimization Problem Statement}

The problem this research is trying to address is as follows: How should a water heater load resource be allocated between three ancillary services, frequency regulation, frequency response and peak demand mitigation, at any given time of the day based on the available energy take among 10,000 water heaters in such a way that scheduling is prioritized to maximize revenue. Revenue is maximized by determining the optimal ratio of ancillary services to provide during each five-minute interval based on market price signals.

\subsection{Why 10,000 water heaters?}

We chose 10,000 water heaters for resource allocation efficiency and speed, and to minimize the computational requirements of the optimizer. While 10,000 is used in the current 
research work, we expect that we will increase the number of EWH in the AWHS as we conduct further research.

An earlier work by Marnell used 100 water heaters in an aggregation system[4]. However, most noticeable with the use of 100 water heaters was the reduction in the energy take of the AWHS, and the granularity of the power in the AWHS. Consistent with the law of large numbers, Marnell observed that the energy take curve of the AWHS got smoother as he increased the number of water heater units. This observation is in line with our expectation. We chose 10,000 water heaters because it is in the medium-size range, i.e., not too few, and not too many.

\subsection{Justification for the use of Linear Programming}

Linear Programming (LP) was chosen because all the parameters used in the AWHS are linear, i.e., the objective function, decision variables and system constraints are all linear. Second, there are no non-linear functions or variables in our problem formulations. Most important of all, linear problems are easier to compute, determine the solution, model, and satisfy the constraints defined. Given the amount of computation involved in this research, and the limitations on computational resources, LP was a reasonable choice for this work.

Although, in academia, there are several methods for optimizing non-linear problems, in practice however, there are not very many inexpensive, non-linear methods available for commercial use. To make matters worse, most of the non-linear solvers used in academia are complex, requiring specific modelling and problem formulation techniques. In addition, 
non-linear programming generally produces different solutions based on the starting points, making the optima very difficult to reproduce. The lack of a deterministic approach to reproduction of this research made non-linear programming a non-viable path for this research.

The only non-linearity observed in this work was the decay function derived from the recovery curve of the AWHS, after each dispatch. This non-linearity was not used to produce the solutions obtained in our optimization algorithm but rather was an outcome our optimization. 


\section{Nomenclature}

\section{Problem Variables}

$a_{i} \quad$ Peak demand price $\$ / k W h$

$b_{i} \quad$ Frequency regulation price $\$ / k W h$

$c_{i} \quad$ Frequency response price $\$ / k W h$

$N_{i} \quad$ Number of units online $n$

$P_{i} \quad$ System Power $k W$

$E_{i} \quad$ System Energy Take $k W h$

\section{System Outputs}

$x_{1} \quad$ Peak demand mitigation energy $k W h$

$x_{2} \quad$ Frequency regulation energy $k W h$

$x_{3} \quad$ Frequency response energy $k W h$

$P_{1} \quad$ Contracted Power for peak demand mitigation $k W$

$P_{2} \quad$ Frequency Regulation Power $k W$ 
$P_{3} \quad$ Frequency Response Power $k W$

$G_{i} \quad$ Revenue $\$$

\section{Constants}

$E_{\max }$ Maximum System Energy $k W h$

$F R G$ Frequency Regulation Constant $k W h / k W$

FRS Frequency Response Constant $k W h / k W$

\section{Other System Parameters}

$t_{0} \quad$ optimization start time minutes

$t_{d} \quad$ duration of dispatch minutes

$t_{\max }$ maximum time minutes

$i \quad$ five minute time intervals $n$

$n \quad$ numeric value 


\subsection{Problem Variables}

\subsubsection{Time interval index, $i$}

There are 288 five minute intervals in any given day. So the range for index $i$ is from 1 at time, $t_{1}$ and 288 at time, $t_{\max }$.

\subsubsection{Service Prices}

The AWHS earns revenue by participating in three ancillary service markets. The market clearing price of each of these services vary in real-time. Future prices are therefore forecasted for the upcoming 5 minute intervals using historical market prices. As time increases, previously forecasted values are updated so that they reflect the most up-to-date prices. The AWHS is a price taker, which means it is unable to influence market prices and it accepts the market prices at the time of bid.

\subsubsection{Peak Demand Price, $a_{i}$}

We use published Locational Marginal Pricing (LMP) data made available by PJM for energy pricing. The LMP is the amount it costs to sell or purchase power at different nodes (locations) within the regional electricity Independent System Operator (ISO). In most markets, the LMP factors in line losses, congestion costs at the priced node and energy prices.

We chose PJM due to the volume and duration of historical data made available to the public, in most cases going back 60 months. In addition, unlike CAISO, PJM services span 
a larger service territory, and PJM readily provides a wide variety of operation data with its user-friendly data miner that is available to its website visitors. ${ }^{15}$

To value peak demand mitigation, we use PJM's Locational Marginal Pricing (LMP) data and apply a multiplier to the LMP price data between 4:00pm and 8:00pm. This multiplier serves to emphasize the importance of this service. For summer and winter months, peak demand mitigation occurs when temperature exceeds $90^{\circ} \mathrm{F}$ or drops below $32^{\circ} \mathrm{F}$ respectively. During the time window, the optimization routine determines when the AWHS should be reserved for Peak Demand based on the LMP and ambient temperature.

The LMP is comprised of three components: the energy price, congestion costs, and power line losses costs. We consider only the energy market in this work, therefore we have elected to treat LMP prices as being equivalent to energy prices.

\subsubsection{Frequency Regulation Price, $b_{i}$}

PJM provides five minute market data for frequency regulation. Frequency regulation is comprised of two parts, regulation capability and regulation performance. Like Opathella et al., we combine these two prices as one [147]. Our choice to combine these two service prices as one is based on the quick response time of resistive water heaters; they are sufficiently responsive to always meet the performance criteria.

FERC Order 755 allows frequency regulation to be adequately rewarded in the wholesale power markets based on capacity payments that includes the marginal unit's opportunity cost and the quantity of frequency regulation service provided by a resource when the

\footnotetext{
${ }^{15} \mathrm{https} / / /$ dataminer2.pjm.com
} 
resource is accurately following the dispatch signal [148]. Previously, frequency regulation payments were only for capacity, whereas the performance for system frequency control was not considered within the regulation market clearing and settlement. Earlier frequency regulation compensation schemes led to operational grid inefficiencies [149]. Prior to FERC order 755 , resources with superior speed and accuracy were significantly more effective at responding to system imbalances, but were paid the same as slower and less accurate resources. ISO markets paid regulation providers a "capacity payment" to make some capacity available in 5-15 minutes and not based on performance (how fast they responded). Now resources are paid on $\$ / \mathrm{MW}$ provided for capacity and the "mileage" performance, where "mileage" is calculated as the absolute value of the sum of the frequency increments or decrements caused by a resource's ability to inject or absorb power.

We use the provided regulating performance prices furnished by PJM. Regulation capability refers to whether a unit is able to respond to PJM's frequency regulation signal or not. Simply stated, regulation capability is the cost of reserving a fixed amount of MW for regulation. The AWHS will allocate a percentage of the total energy take available for frequency regulation, as determined by the optimization algorithm.

\subsubsection{Frequency Response Price, $c_{i}$}

There is currently no market for frequency response in the U.S. Nevertheless, we structure its pricing model as a constant, which signals a steady-state need for reserving this service. We chose to model frequency response pricing as a constant because we found other literature where frequency response has been similarly modelled. For example, O’Sullivan et al. used 
a fixed frequency reserve constraint in their economic dispatch models for smaller municapal utilities [150]. For simplicity of their model, Ela et. al used a hypothetical optimization that assumed frequency response bids are constant [151, 152].

TIME OF DAY VS. DATE OF FREQUENCY RESPONSE EVENT

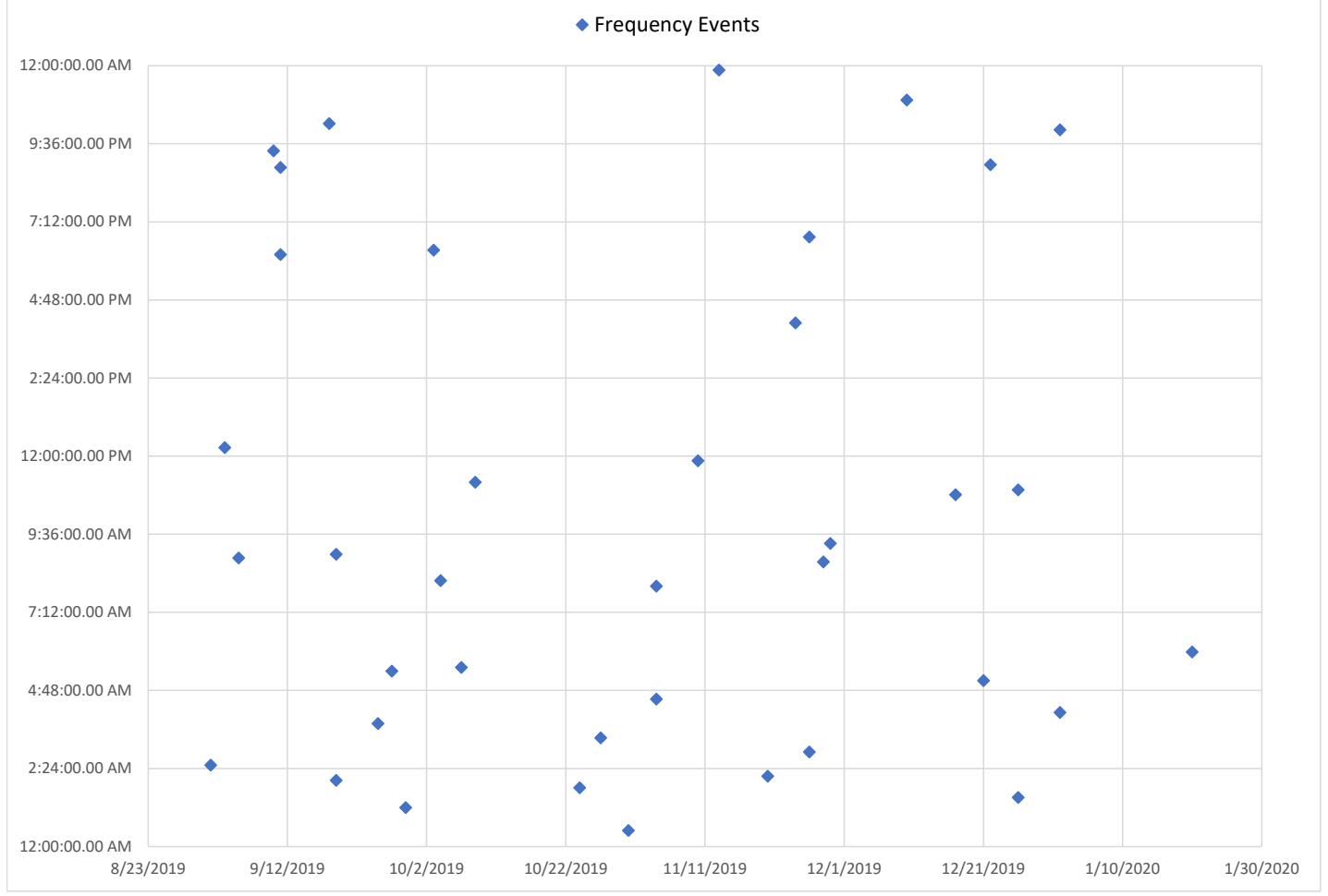

Figure 4.1: Frequency response events recorded at the Salem Smart Power Center from August 23, 2019 January 31, 2020, Source: PGE.

Frequency events recorded at PGE's Salem Smart Power Center between August 2019 and January 2020 are presented in Figure 4.1. Frequency response events are not correlated with any predictable phenomena like weather, transmission congestion, or distribution system overloading. Therefore, the price is justified to be a constant. If system operators are 
not able to predict when a frequency response event will occur, price cannot be increased or decreased in anticipation of bringing additional response resources online.

\subsubsection{System Energy Take, $E_{i}$}

The available energy take in the AWHS depends on the states that the individual water heaters are in at any given time. The state diagram shown in Figure 4.2 is a variation of the one that was developed by Marnell [4].

There are three states. In states 1 and 3, the AWHS controls how individual water heaters in the system manage their energy take. In state 2, the AWHS relinquishes control to the individual units for the purposes of improving occupancy comfort and prioritizing the residents' water heating needs. As shown in Figure 4.2, the temperature of the water in each unit informs the three states and determines the total amount of energy take available in the system at any particular time. 


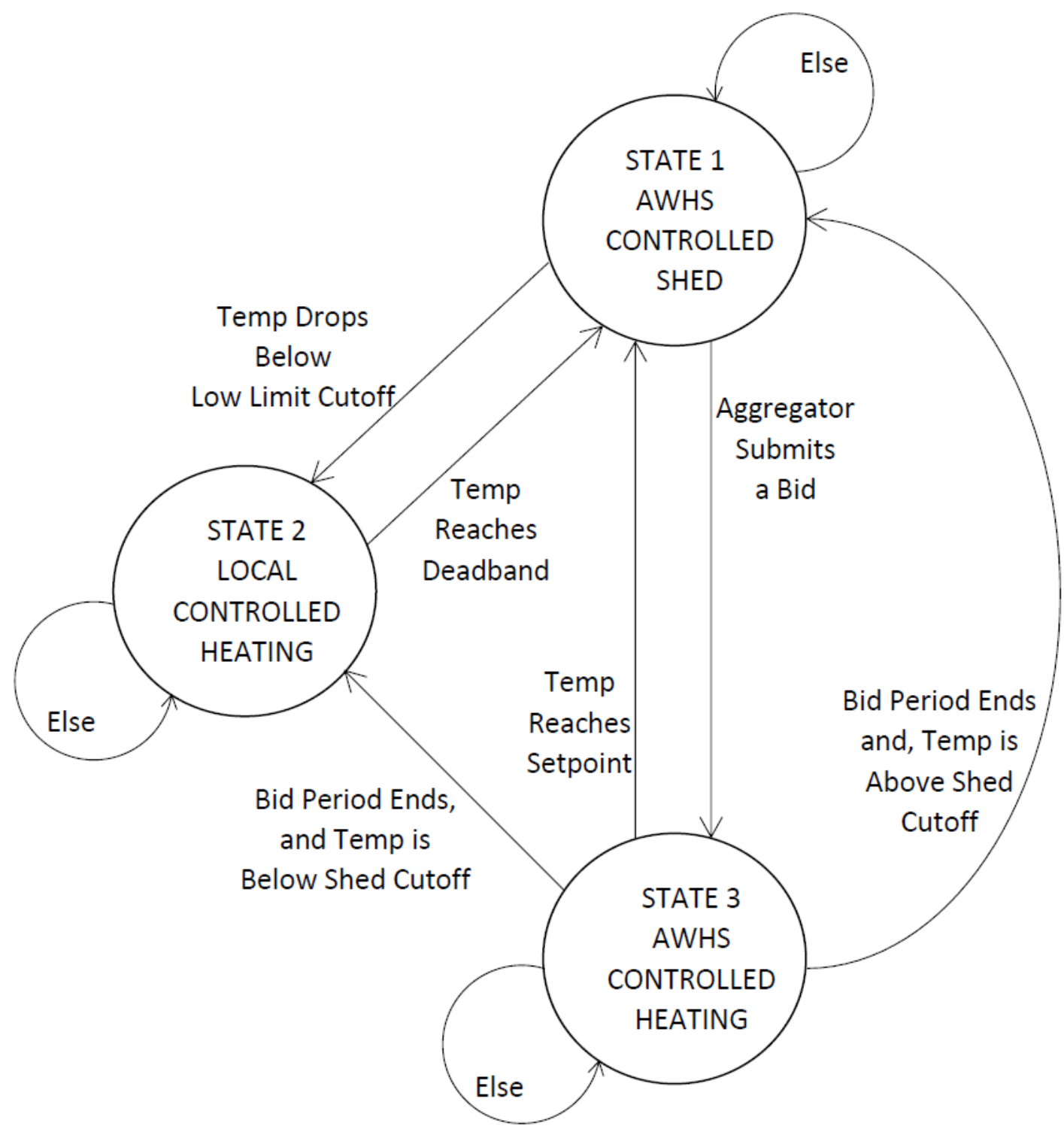

Figure 4.2: State diagram for electric water heater model [4].

\subsubsection{Power, $P_{i}$}

The maximum power capacity of the system is derived from the available energy per time step. Each water heater has a maximum rated power of $4.5 \mathrm{~kW}$, therefore, the maximum power available in the system can not exceed $45 \mathrm{MW}$, which would only occur if all 10,000 
units were available.

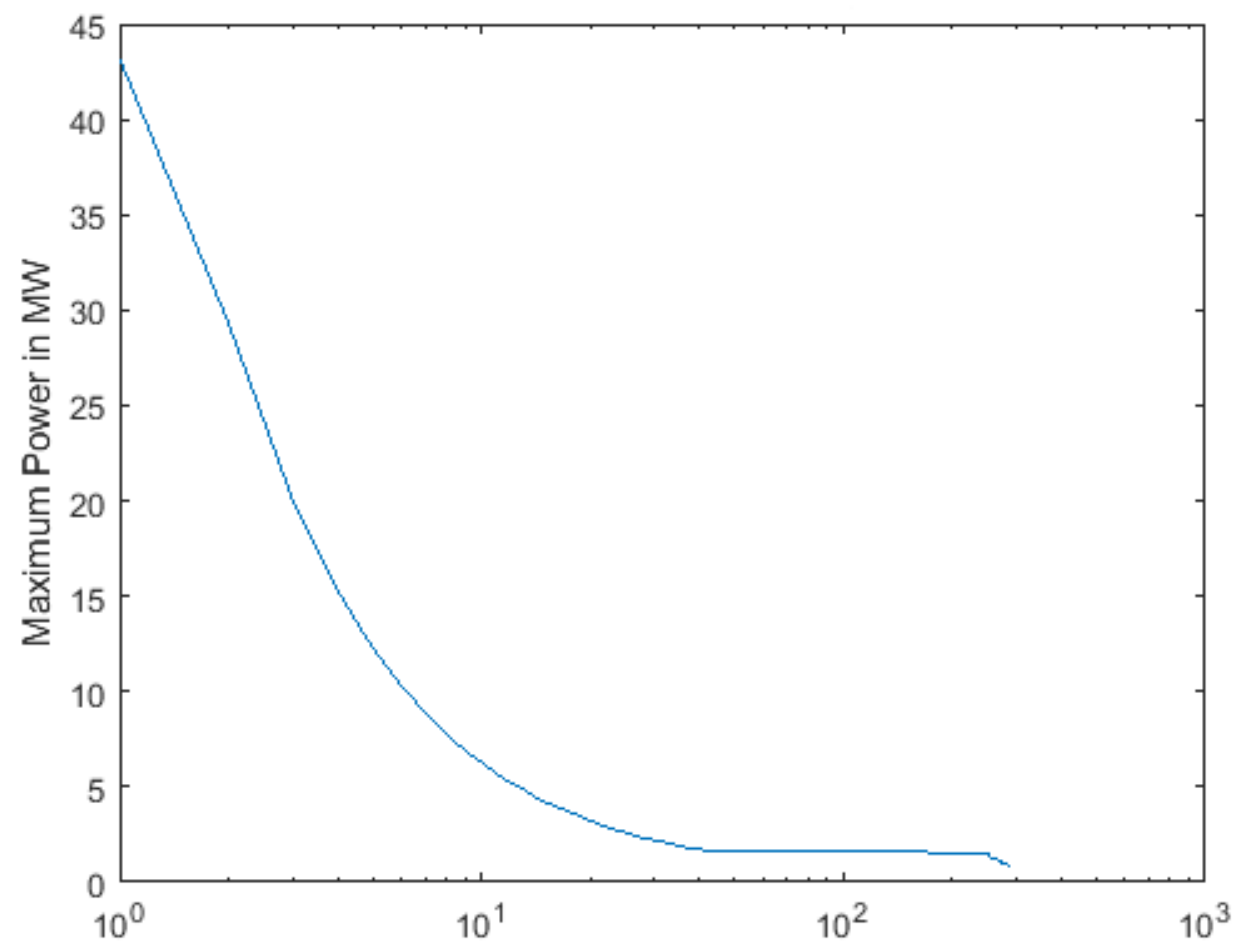

Figure 4.3: Maximum power available in the AWHS vs. the number of consecutive five-minute dispatches [4].

In Figure 4.3, we plot the maximum power available in the AWHS vs. the number of consecutive five-minute dispatches. This figure answers the question of how many dispatches can be made consecutively, at a fixed power level, without running out of energy take capacity. As the bid interval length increases, the available maximum continuous power from the AWHS decreases. For one bid in any given 24 hour period, the AWHS is power limited and can only supply 43 MW per per five minute of energy, as shown on the far left [4]. It is never the case that all the water heaters in the AWHS are available at any given time. In our research, we have observed that the AWHS can successfully consume up to 2.0 
MW continuously without ever running out of energy. This is equivalent to $0.17 \mathrm{MWhr}$ per five minute interval.

\subsubsection{Revenue Earned, $G_{i}$}

The revenue earned by selling reserve capacity and dispatch bids at time interval $i$ is represented by $G_{i}$.

\subsubsection{Number of units, $N_{i}$}

$N_{i}$ represents the number of units available at time interval $i$. Although there are 10,000 water heaters in the AWHS, depending on the particular dispatch requirements of the aggregation system, the optimization algorithm, and the available energy take in the AWHS, the number of units available to respond to a bid at any given time will vary. 


\subsection{Decision Variables}

The objective of this research is to optimally decide among three services what proportion of each service should be deployed at any given time interval, $t_{i}$, that will yield the most revenue, within any given 24 hour window. Each of the three services are represented, by the subscripts 1,2 , and 3 of the letter $x$, for peak demand mitigation, frequency regulation, and frequency response, respectively. In other words, $x_{1}=$ peak demand mitigation, $x_{2}=$ frequency regulation, and $x_{3}=$ frequency response. Our optimization algorithm solves for the value of $x$ for each of the three services that maximizes the revenue of the AWHS.

\subsubsection{Peak Demand Mitigation Energy, $x_{1}$}

$x_{1}$ is the amount of peak demand energy scheduled as a reserve during peak demand hours. This is the amount of energy that will be held back from being dispatched into the energy market.

In general, electricity consumption reaches peak capacity less than 20 times a year and for brief intervals that last about two to three hours. During these periods, demand response programs such as peak demand mitigation are used to reduce peak consumption. These periods are defined by time of day and ambient temperature, as described in 4.2.2.1. The AWHS will not dispatch its load resources during peak demand mitigation periods. The AWHS will be compensated by the amount of energy it is able to hold back in reserve during peak demand mitigation.

PJM has two five minute reserve markets based on geographical locations, namely, 
the Mid-Atlantic Dominion (AD) reserve market and the Regional Transmission Operator (RTO) reserve market. Further, PJM classifies reserves into two types, synchronized and non-synchronized reserves. Synchronized reserves are from generators or certain demand response loads that can provide relief to the grid within 10 minutes. Non-synchronized reserves refers to resources (or loads) that can respond within 10 to 30 minutes. The water heaters in the AWHS are considered online (connected to the grid) and can respond in a matter of a few seconds, and as such, they meet PJM's synchronized reserves market participation requirements.

\subsubsection{Frequency Regulation Energy, $x_{2}$}

$x_{2}$ is the amount of dispatchable energy available in the AWHS for frequency regulation. Frequency regulation services match generation to load so as to maintain the frequency of the grid near $60 \mathrm{~Hz}$ at all times. The AWHS uses water heaters for participation of frequency regulation services.

Regulating units correct for small changes in load or generation that cause the power system to operate out of balance. PJM's five minute market has two frequency regulation price structures based on the performance and capability of the provider, namely, "RegA" and "RegD". The former is a lowpass signal for slower resources while the later is a high pass signal for more dynamic, faster resources. Water heaters respond very quickly, so they are well suited for responding to RegD signals. This was demonstrated by Slay, who 
showed that resistance water heaters can accurately follow PJM's RegA and RegD frequency regulation signals [153].

\subsubsection{Frequency Response Energy, $x_{3}$}

$x_{3}$ represents the energy reserved in the AWHS for frequency response. As RER continue to displace traditional synchronous generators, the total inertia in the electric grid continues to decrease owing to the decoupling of the mechanical rotor speed and system frequency. RERs like wind and solar PV do not have sufficient inertia to help mitigate system frequency fluctuations, which can lead to instability of the grid frequency and reduce the ability of the power system to remain stable following a system disturbance.

The equation below describes the relationship between power and frequency [154]:

$$
\triangle f=\frac{1}{M_{\mathrm{s}}+B}\left(\triangle P_{e}-\triangle P_{i m p}\right)
$$

$\mathrm{F}$ =average frequency change

$M_{s}=$ System inertia of the system

$\mathrm{B}=$ is the inverse of the frequency sensitivity

$P_{e}=$ is the change in power exported

$P_{i m p}=$ is the change in power imported

$\beta=$ Power-Frequency ratio

For a perfectly balance system, with zero system imbalance[154]:

$$
\beta=\frac{\triangle P_{e}}{\triangle f}(M W / 0.1 H z)
$$


Traditionally, generation is used to maintain system frequency. For any reduction in frequency, generation must be ramped up to restore system frequency, and likewise, if the frequency rises, generation must be ramped down. Alternatively, this can achieved by controlling loads. There exists a direct correlation between system frequency and available loading. The loads available in the AWHS can be used to respond to system frequency calls.

Frequency response is called upon when there is a sudden unacceptable fluctuation in grid frequency. For example, such events can occur during the unplanned loss of a large TGR that causes the frequency to rapidly decrease. In order to restore the nominal frequency, real power must be quickly provided to the grid. To maintain a constant frequency, the active power supply must be equal to the load at any given time. Most TGR have active power reserves that must be quickly brought on-line after seconds of imbalance so as to prevent the system frequency from changing too much. The AWHS can only help with grid frequency increases by bringing more water heater loads online. Therefore, the AWHS can be used as a frequency response load resource for addressing sudden frequency increases.

\subsection{Optimization Parameters Definitions}

We construct the mathematical formulation for our optimization algorithm in the following subsections:

\subsubsection{Objective function}

The objective function is a mathematical model that describes the ratio of services that result in the maximization of revenues with respect to each of the three services under 
consideration.

The maximization problem for revenue $\mathrm{G}$, over $i$ then becomes;

$$
\max _{x 1, x 2, x 3} \sum_{i=1}^{i=288} G\left(a_{i}, b_{i}, c_{i}, x_{1 i}, x_{2 i}, x_{3 i}\right)
$$

$x_{1 i}, x_{2 i}$, and $x_{3 i}$, are the scheduled energy take for peak demand mitigation, frequency regulation, and frequency response respectively.

Since,

$$
x_{1 i}=P_{1 i} \triangle t_{i}
$$

and

$$
x_{2 i}=P_{2 i} \triangle t_{i}
$$

and

$$
x_{3 i}=P_{3 i} \triangle t_{i}
$$

the revenue, $G_{i}$ from equation 4.3 can be written in terms of $P_{i}$ as follows:

Revenue from peak demand mitigation is:

$$
G_{1}=\sum_{n=i}^{i=288}\left(P_{1 i} a_{i}\right) \triangle t_{i}
$$

Revenue from frequency regulation; 


$$
G_{2}=\sum_{n=i}^{i=288}\left(P_{2 i} b_{i}\right) \triangle t_{i}
$$

and Revenue from frequency response is;

$$
G_{3}=\sum_{n=i}^{i=288}\left(P_{3 i} c_{i}\right) \triangle t_{i}
$$

so the objective function, and maximum revenue becomes;

$$
G_{\text {total }}=\sum_{i=1}^{i=288}\left(P_{1 i} a_{i}+P_{2 i} b_{i}+P_{3 i} c_{i}\right) \triangle t_{i}
$$

\subsubsection{Optimization Constraints}

\subsubsection{Available Power}

We use LP to solve for the optimal Power $P_{1}, P_{2}$, and $P_{3}$ for peak demand mitigation, frequency regulation, and frequency response respectively. The maximum available power capacity, with all units turned on can not exceed $45 \mathrm{MW}$ if there are 10,000 units with each heating element rated at $4.5 \mathrm{~kW}$

$$
\begin{aligned}
& P_{i}=P_{1 i}+P_{2 i}+P_{3 i} \\
& P_{\text {max }_{i}} \leq N_{i} 4.5 k W
\end{aligned}
$$


where $N_{i}$ is the number of units available at interval $i$, and

$$
P_{\text {max }_{i}}=\frac{\triangle E_{\text {system }_{i}}}{t_{i}}
$$

where $\triangle E_{\text {system }_{i}}$ is the maximum change in the system energy take during time interval $i$.

For the lower bound,

$$
P_{\operatorname{mini}}=0 M W
$$

and

$$
P_{\text {mini }} \leq P_{i} \leq P_{\text {maxi }}
$$

Frequency response is constrained by the available power in the AWHS, and as such, the system is power limited with regards to frequency response, therefore, Equation 4.12 can be written in terms of frequency response as:

$$
P_{3 i \max } \leq N_{i} 4.5 k W
$$

where,

$$
P_{3 i \max } \triangle t \leq E_{i}
$$




\subsubsection{Available Energy}

The energy scheduled for dispatch at any time interval $t_{i}$ shall not exceed $E_{i}$, the maximum available dispatchable energy at any given time.

Where;

$$
E_{i}=\sum_{i=1}^{i=N} E_{n}
$$

where $\mathrm{N}=10,000$ units.

From section 4.4.4, the upper bound of frequency regulation energy, $x_{2}$, a dispatchable service, is constrained as follows:

$$
x_{2 i} \leq 0.17 M W h r
$$

so,

$$
P_{2 i} \leq 2.0 M W h r
$$

We set the upper boundary of frequency regulation to $0.17 \mathrm{MWhr}$ (or 2.0 MW per five minute interval) because from Figure 4.3, at this limit, the AWHS energy regeneration is high enough to sustain multiple consecutive energy takes throughout the day without running down the system energy take. 


\subsubsection{Peak Demand Mitigation}

Peak demand mitigation is constrained to the hours between 4:00pm and 8:00pm during summer and winter months when temperature exceeds $90^{\circ} \mathrm{F}$ or drops below $32^{\circ} \mathrm{F}$ respectively.

\subsubsection{Optimization Equation}

The optimization problem, revenue earned by the AWHS during time interval $i$, can be represented by the following equation:

$$
G_{i}=\left(a_{i} P_{1 i}+b_{i} P_{2 i}+c_{i} P_{3 i}\right) \triangle t_{i}
$$

which then becomes;

$$
G\left(a, b, c, x_{1}, x_{2}, x_{3}\right)=a_{i} x_{1 i}+b_{i} x_{2 i}+c_{i} x_{3 i}
$$

The objective function formulation and optimization therefore becomes:

$$
\begin{gathered}
\text { Maximize } \sum_{i=1}^{i=n} G\left(a_{i}, b_{i}, c_{i}, d_{i}, P_{1 i}, P_{2 i}, P_{3 i}, P_{4 i}\right) \\
\text { for } n=288, \text { and } i_{1}, i_{2}, \ldots, i_{288}, \\
\text { subject to, } \\
P_{\text {min }} \leq \triangle P_{\text {system }} \leq P_{\text {max }}
\end{gathered}
$$

where, 


$$
\triangle P_{\text {system }}=\frac{\triangle E_{\text {system }}}{\triangle t}
$$

and,

$$
P_{1 i}+P_{2 i}+P_{3 i}+P_{4 i} \leq P_{\max }
$$

\subsubsection{Energy take decay solution}

The resource recovery curve represents the energy take left in the AWHS immediately following a dispatch, and it shows the duration that it will take the AWHS to get back to its pre-dispatch energy-take level.

In terms of maximizing profits for the AWHS, there is an opportunity cost lost during the recovery window, since the AWHS will not be able to participate in the energy market as it recovers from its last dispatch.

The energy take due to a dispatch can be derived using:

$$
x_{t+1}=x_{t}-x_{t} e^{-K[(t+1)-(t)]}
$$

We solve for $\mathrm{K}$ using the given equation below,

$$
K=\ln \left(\frac{x_{t}}{x_{0}}\right) t^{-1}
$$

where $\mathrm{K}$ is the time decay constant, $x_{0}$ is the energy take of the AWHS at time 0 , the AWHS dispatch time, and $x_{t}$ is the energy take of the AWHS at time $t$, the time when the AWHS fully recovers. 


\subsection{Optimization flow chart}

Figure 4.4 is a summary of the optimization algorithm work flow. We start with the U.S household water usage program developed by NREL. This program determines water heater draws based on the house hold sizes. The water heaters are assigned states as discussed in 4.4. We simulate the energy take for 1 year. From this large repository of data, a subset can be extracted for any month, day, hour or minute within the 1 year window. Due to the large data set size, we only consider a small segment of the data at any given time, $[\mathbf{X}]$, four weeks worth of data specifically. We then we proceed with stage 1 optimization, where all services are treated as if there were all reserve services. $[\mathbf{X}]$ is then updated with data, informed by stage 1 optimization, which is then re-sorted by the state of the water heaters. This updated data are then used for the second stage optimization. The program terminates when $t_{i}$ equals to 288 . 


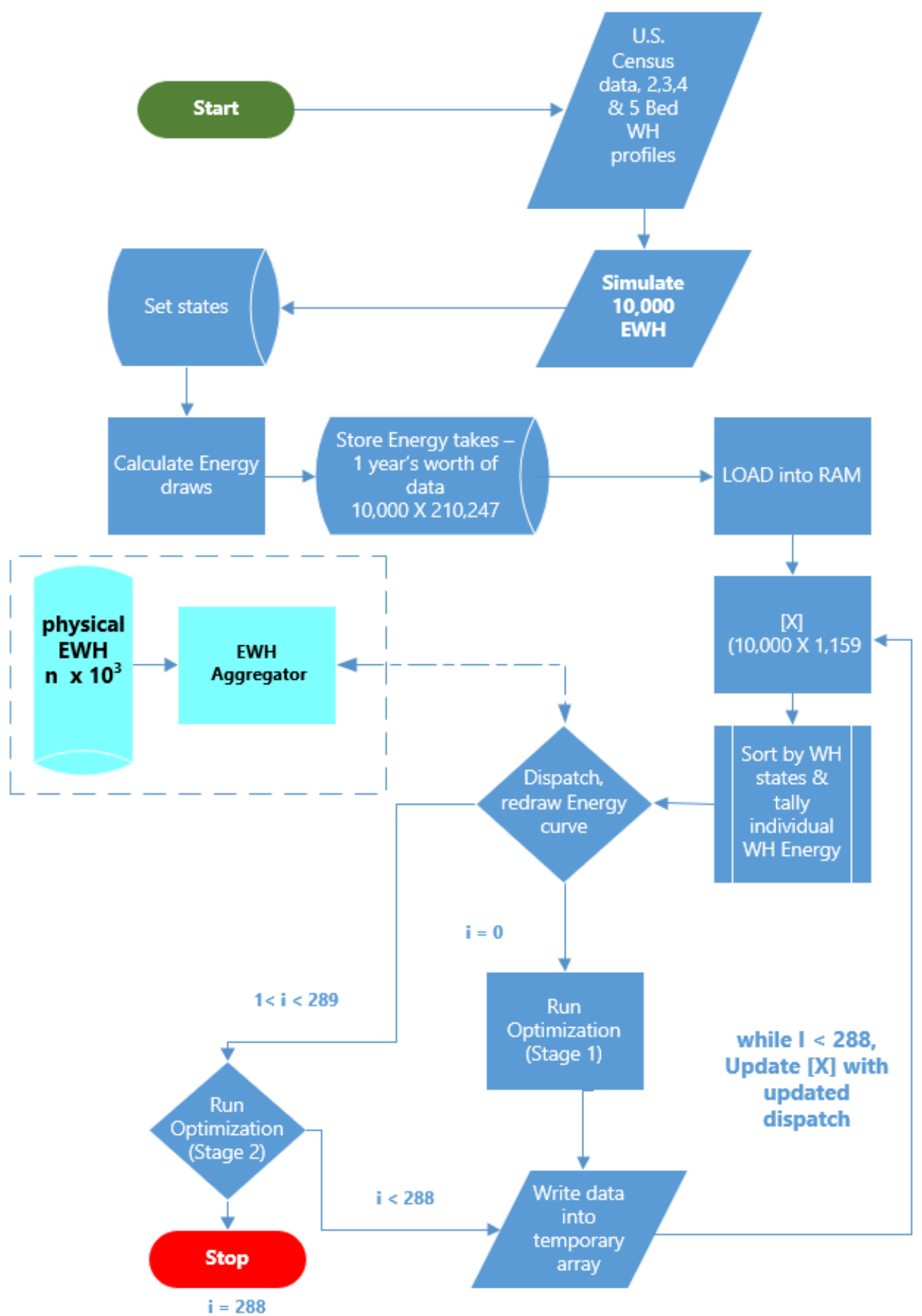

Figure 4.4: AWHS optimization process flow chart. 


\section{Results \& Analysis}

In this chapter, we report the findings of our study based upon the methodology described in chapter three. This chapter is divided as follows: In section 5.1, we provide results of our research using a baseline AWHS resource with no dispatch. Our baseline simulations are the starting point that will be used in our comparisons to the dispatch scenarios, and for further analysis of our results. In section 5.2, we dispatch short fixed amounts of energy in order to understand the operation and response of the AWHS. We do this to better understand the limits of the AWHS resource. These dispatches are carried out in five-minute intervals, one dispatch at a time as shown. In section 5.3, we perform multiple AWHS dispatches. In section 5.4, we analyse the performance of the AWHS over prolonged dispatch periods. In section 5.5, we discuss the use of LSTM for forecasting future data. An in-depth analysis of the optimization is presented in sections 5.6 - 5.8. We compare the revenues generated by out AWHS optimization algorithm to that of the AWHS without our optimization algorithm in section 5.9. The remainder of this chapter is dedicated to discussing the value of including other ancillary services, our choice of LP constraints, other research objectives, and finally, we conclude with the shortcomings of this work in section 5.13. 


\subsection{Baseline AWHS overview}

This section discusses the observed behavior of the AWHS when it is not perturbed; we examine how the AWHS behaves when it is not being dispatched. Figure 5.1 shows a typical energy take profile over one day. The energy take present in the system is represented on the vertical axis while the five-minute intervals are represented on the horizontal axis. There are two peaks, with the earlier peak occurring in the AM hours, and the second peak occurring in the PM hours. This is consistent with typical water heater usage profiles. These peaks both correlate to expected energy draw times. 


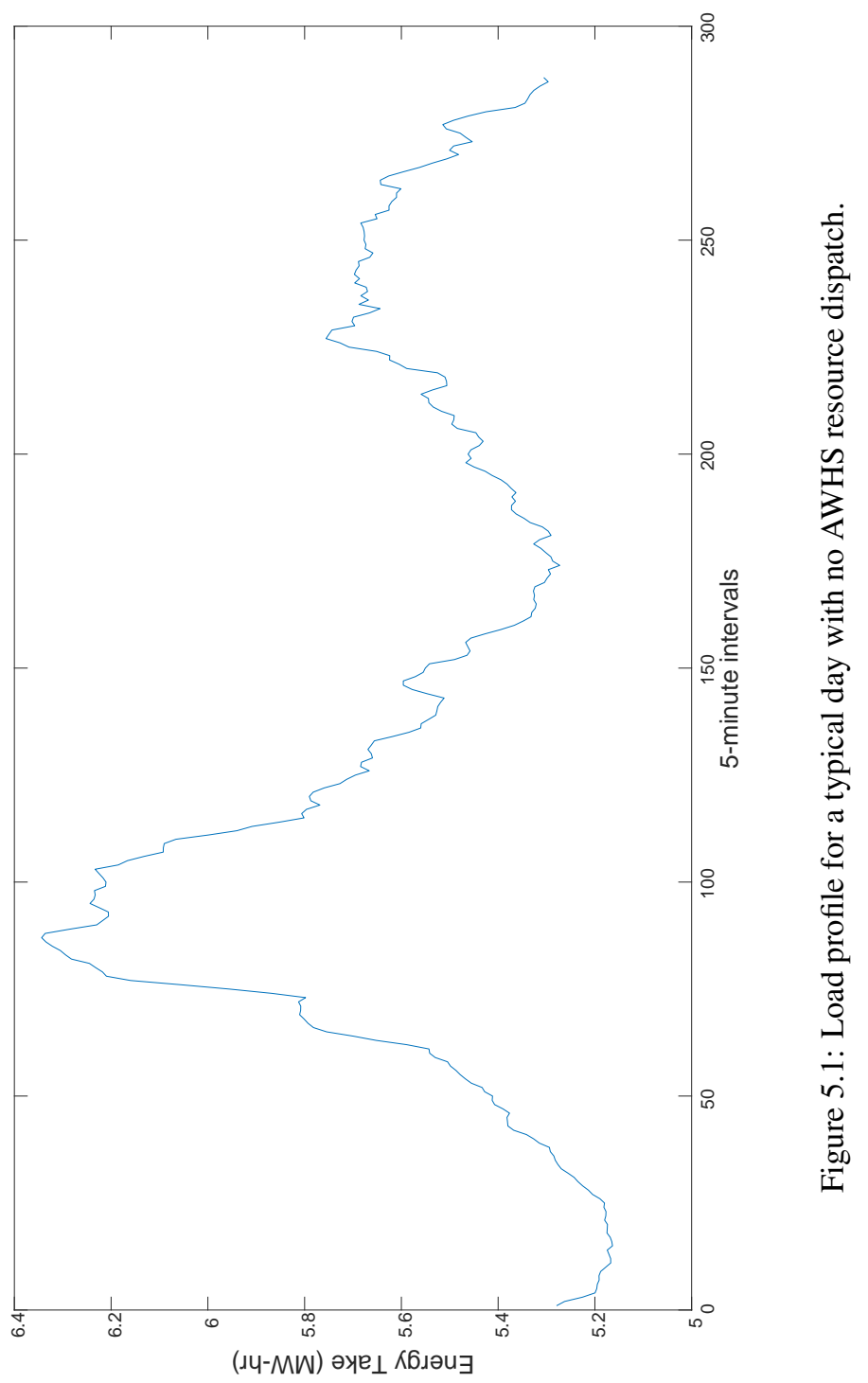


A water heater unit turns on when the amount of hot water drawn out of the tank causes the temperature of the water in the tank to fall below a threshold temperature, due to additional cold water replacing the withdrawn hot water, and causing the heating element to turn on. This threshold temperature is not standardized. It varies between water heater manufacturers.

Figure 5.2 show a typical energy take profile over five days. These baseline simulations represent the energy take of the AWHS on average days, with the AWHS not bidding into any ancillary service markets. 


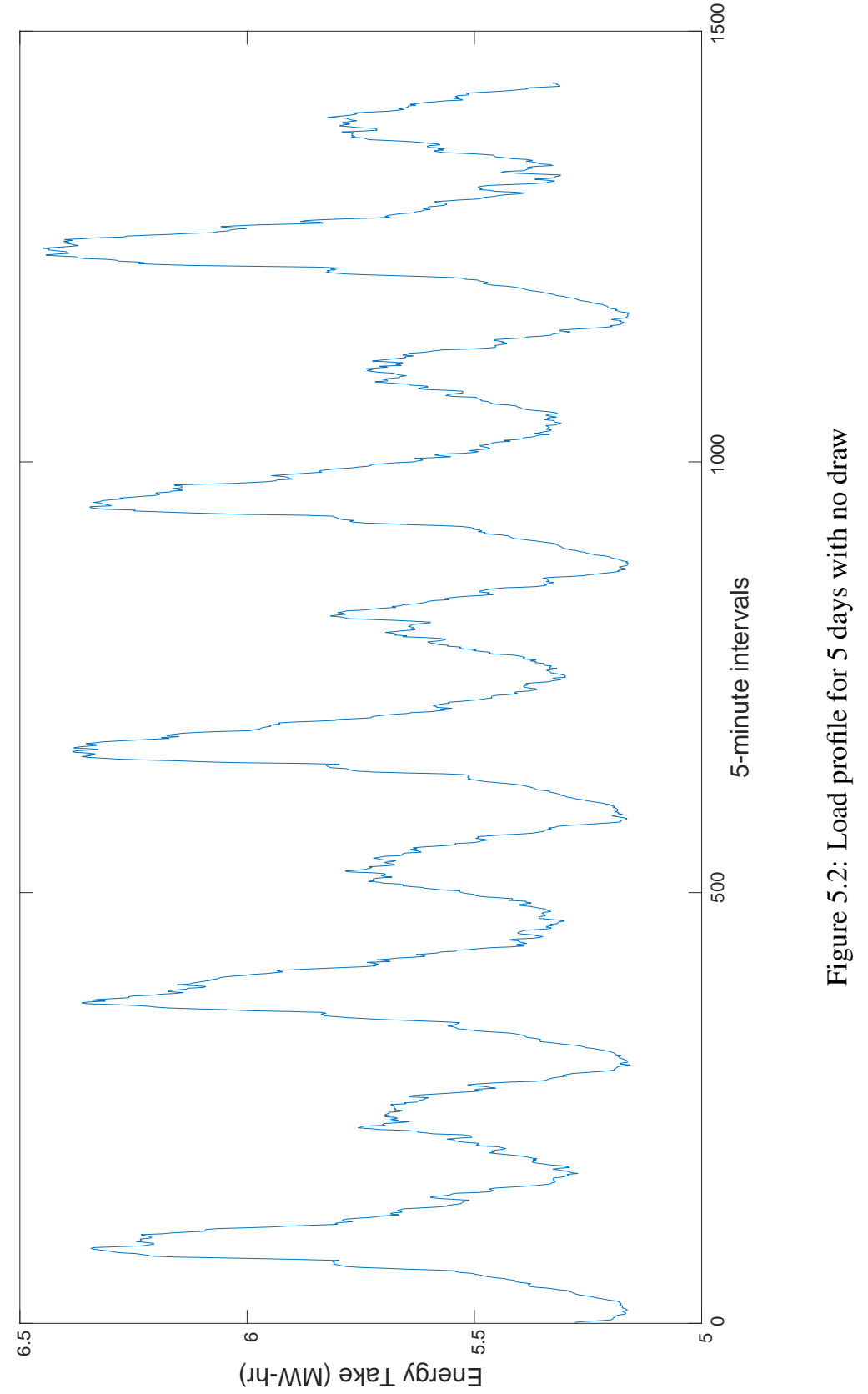




\subsection{Single AWHS dispatches}

In Figure 5.3, the AWHS makes a single, one time, five-minute, $5 \mathrm{MW}$ bid, at 0200 . The Available energy in the AWHS dips to $3.75 \mathrm{MW}$-hr following this bid. It takes the AWHS about six hours to recover. The dotted line between 0200 and 0800 shows the energy profile of the AWHS if we had not dispatched the AWHS. 


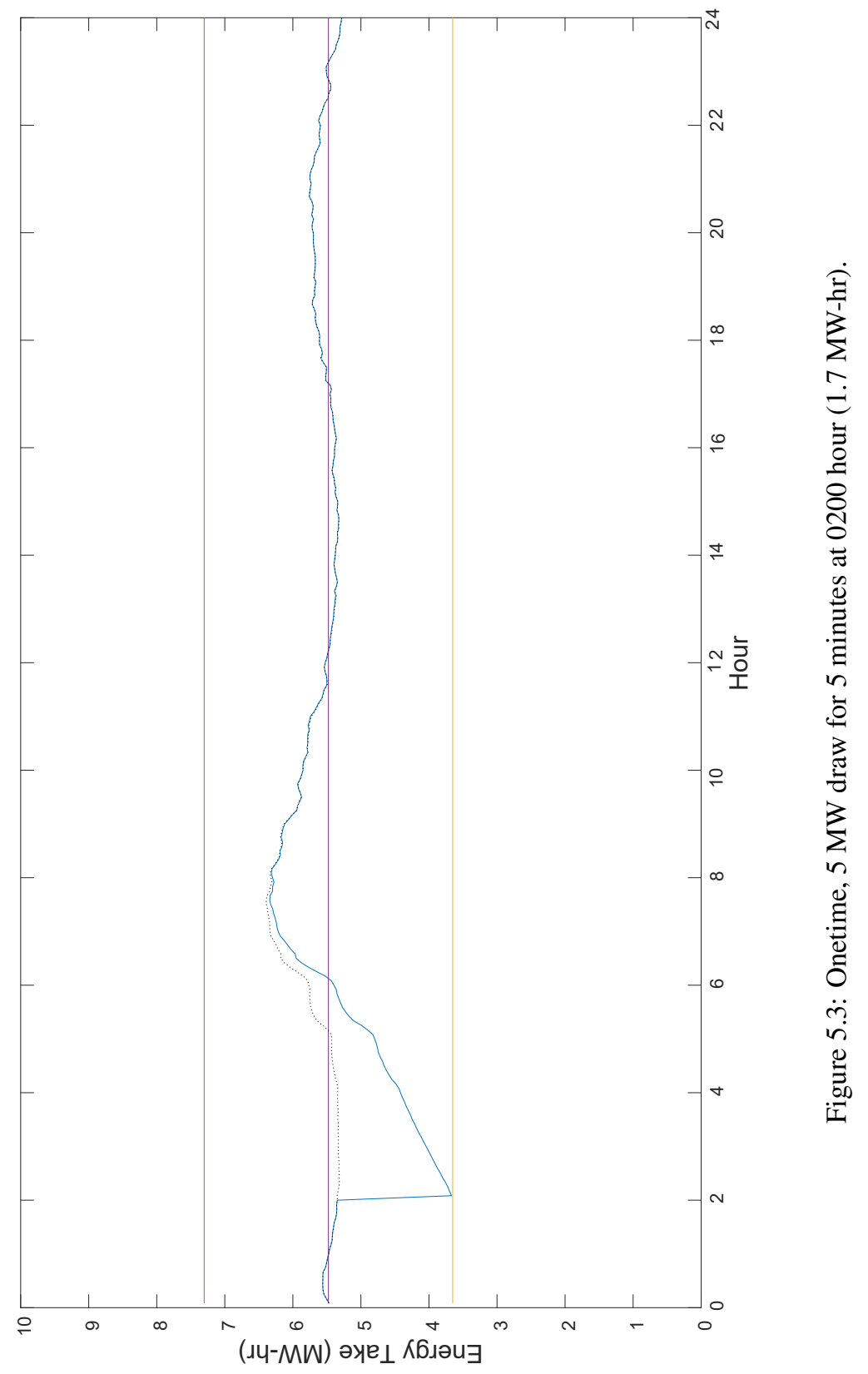


If all units in the AWHS were operating at their highest temperature setpoint (e.g. all units at $120^{\circ} \mathrm{F}$ ), there will be no energy take available.

The yellow line in Figure 5.3 represents the upper bound of the system during the "shed mode". This represents the minimum energy take ever available (e.g. all units at $117^{\circ} \mathrm{F}$ ). The upper bound represented by the red line results from the lower temperature band during the shed mode. The red line represents the maximum energy take possible within the system (e.g. all units at $114^{\circ} \mathrm{F}$ ). In other words, the upper boundary, in red represents the energy take of the AWHS if all units were at their lowest temperature setpoint. The purple line is illustrative of the median energy take of the AWHS.

Figure 5.4 shows a sudden spike in the amount of units that are turned on in order to accommodate the $5 \mathrm{MW}$ bid at hour 0200 . This sudden spike in the percentage of units turned on is anticipated since we are interested in having the AWHS respond as quickly as possible. In this case, $48 \%$ of all the water are turned on to respond to this $5 \mathrm{MW}$ bid. The system does not settle until around 0800, six hours after the 0200, five-minute $5 \mathrm{MW}$ dispatch. The dotted line in Figure 5.4 represents the behavior of the AWHS if we had not performed the dispatch, while the solid line with the spike represents the percentage of water heaters in the AWHS that were turned on to support the earlier dispatch in Figure 5.3. 


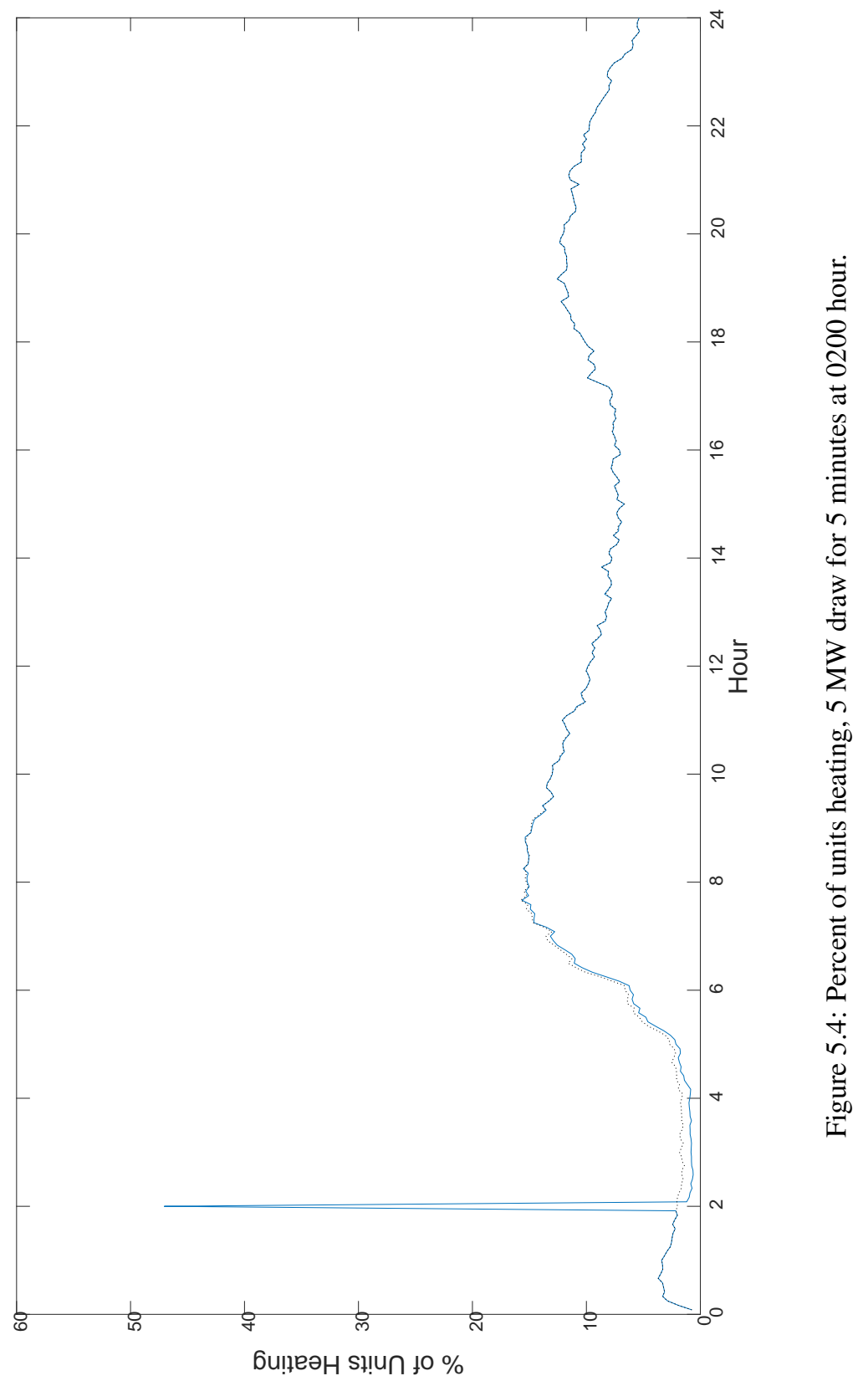


In Figure 5.5, we test the limits of the AWHS by simulating $20 \mathrm{MW}$ of energy for 60 minutes from 0200 to 0300 . In this instance, the system does not recover until 1100 (8 hours after the end of the dispatch). The underlying dotted line between $0200 \mathrm{hr}$ and 1100 hr shows the energy profile of the AWHS if it had not been dispatched on this day. We conducted these simulations to show what a failed dispatch looks like: the AWHS runs out of energy during an unsuccessful $20 \mathrm{MW}-\mathrm{hr}$ bid. The system does not fully recover until 1000, when the dotted line merges with the solid line. 


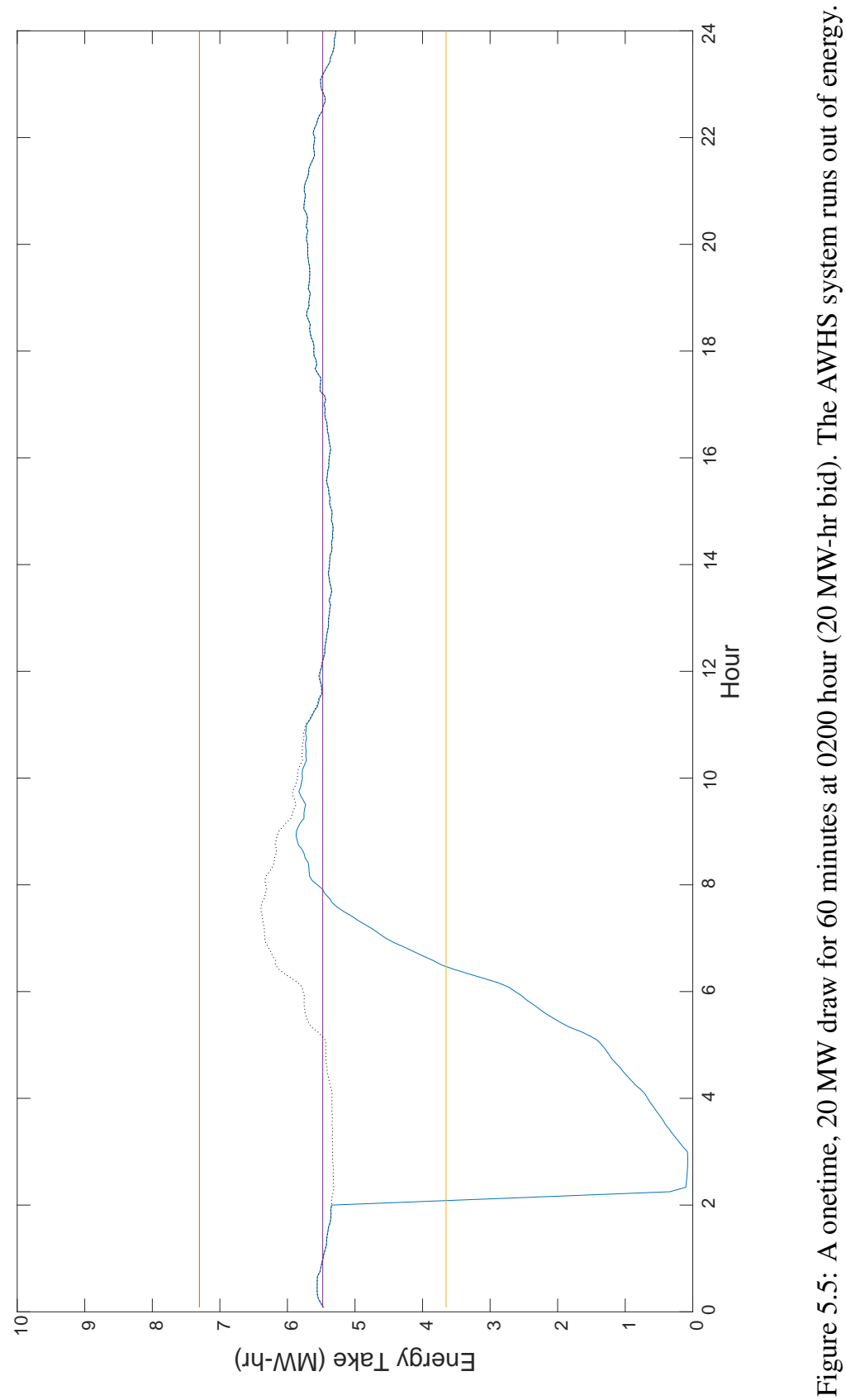


Figure 5.6 shows that the AWHS turned on all units to respond to this $20 \mathrm{MW}$-hr bid before running out of energy at $0300 \mathrm{hr}$. 


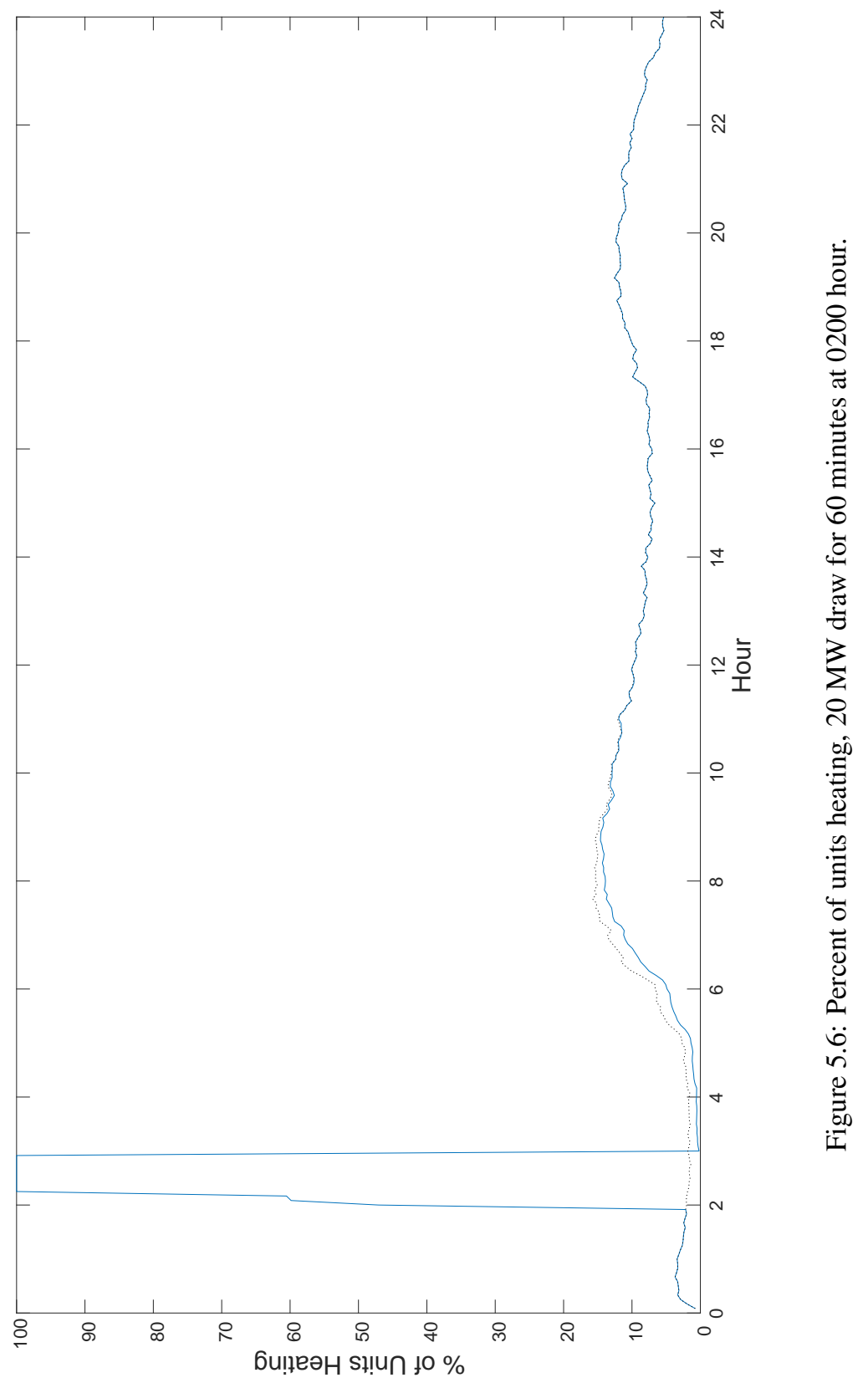




\subsection{Multiple AWHS Dispatches}

Next, we performed multiple single dispatches in order to further understand the behavior of the AWHS. This is important for our purposes because the AWHS will be dispatched multiple times during a day, based on the system's ability to maximize revenue.

As shown in Figure 5.7, we placed three separate bids. The first bid is a five-minute bid at 0100 for $12 \mathrm{MW}$. We then allow the system to recover for an hour. Then again at 0200 and 0300, we place two additional $12 \mathrm{MW}$, five-minute bids. As can be seen in Figure 5.8 the system tries to recover to its pre-bid state but does not have enough time to merge with the dotted line before the next bid occurs at 0200 and 0300 . Figure 5.8 shows the dynamic nature of the AWHS energy take resource, and the need to account for a recovery time when making multiple bids throughout the day, especially since each bid will be based on market signals that sometimes may be very favorable and other times not. 
Figure 5.8 shows the percentage of water heaters that respond to the water heater bids. The percentage of water heaters that are turned on in the system increases during each five minute draw for the three $12 \mathrm{MW}$ draw for 5 minutes each, starting at 0100,0200 and 0300 . As expected, the percentage of the units in the AWHS drops at the end of each bid. The dotted line represent the normal percentage of units that would have been turned on by the AWHS for the day if we had not dispatched the AWHS. The percentage of units turned on per draw is approximately $25 \%$ of the total 10,000 units within the AWHS. 


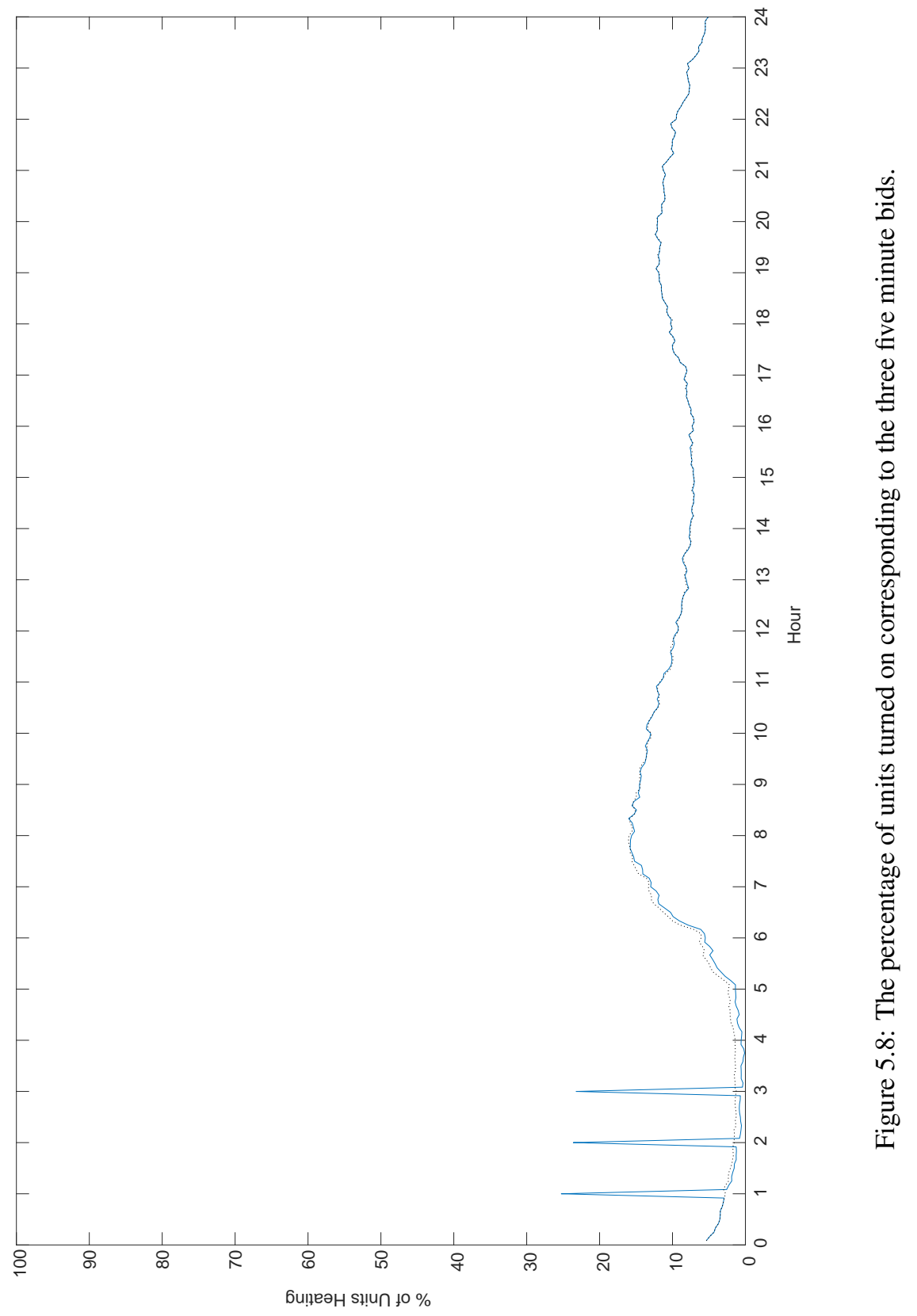




\subsection{Prolonged AWHS dispatches}

TGR are committed to providing energy for blocks of time at a fixed power output. For the AWHS however, the available energy take resource within the AWHS is constantly changing. The dynamic nature of the units that make up the AWHS causes the energy take available for bidding to fluctuate at any given time. Further, as we have demonstrated in Figures 5.3 to 5.8, it is important that we re-evaluate the available energy left over in the AWHS after each dispatch. The dispatch routine does the evaluation, and factors it into the next dispatch.

In Figure 5.9, we simulate a continuous 12 hour dispatch, from hour 0030 to 1330 . The system continues this re-evaluation and re-dispatching until the last dispatch call, in this case, at 1330. The system does not recover back to its pre-dispatch state until 2000 hour. Between 1330 and 2000, the system is not available to fully participate in the market while it recovers to its full capacity. 


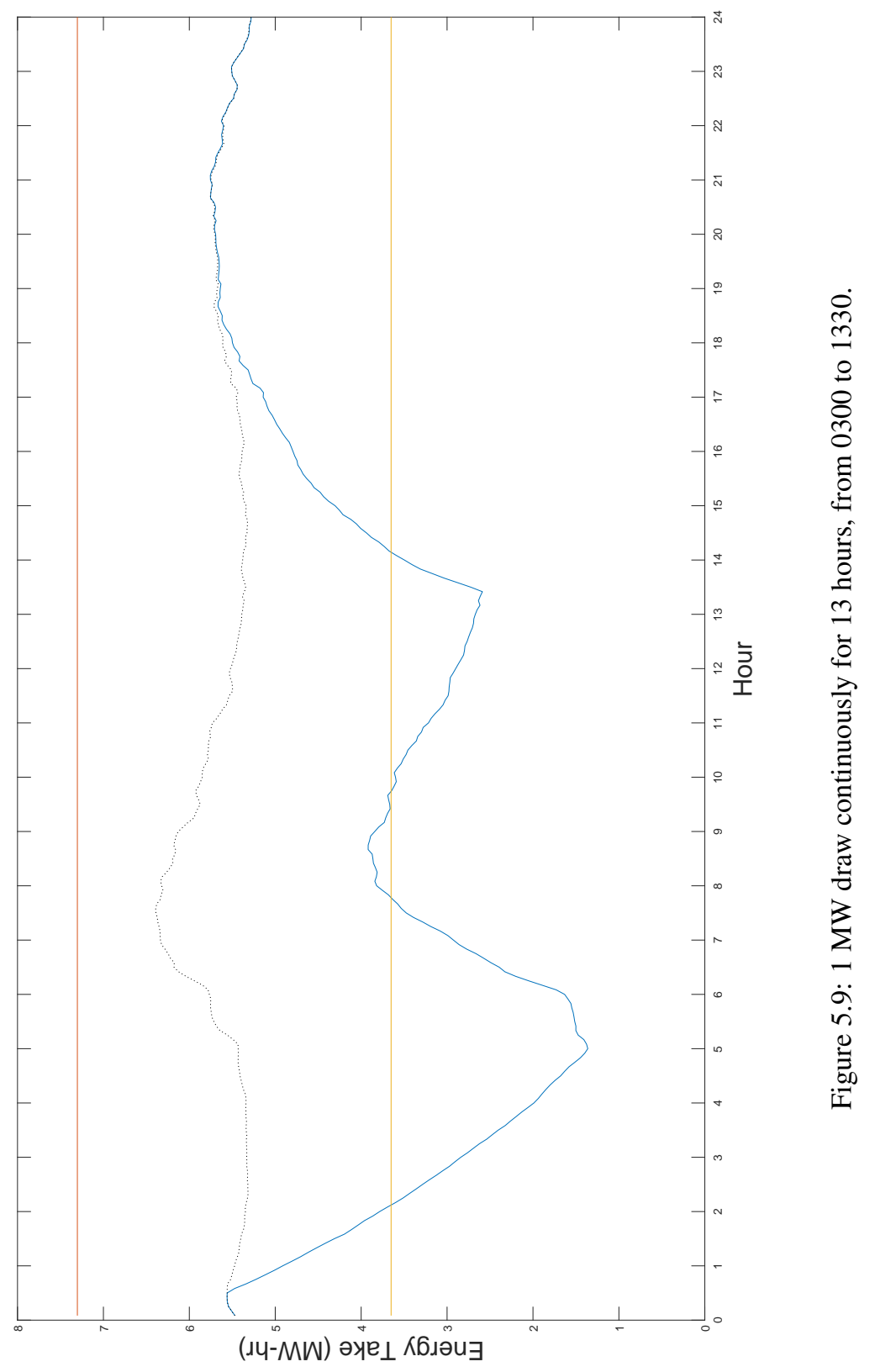


Figure 5.10 shows a continuous 24 hour, 1 MW bid. The dotted line represent the AWHS if we had not bid into the AWHS. At the onset of the AWHS bidding into the market, the available energy in the AWHS decreases up until about 0500 when the available energy in the system flattens, for about an hour, and then the energy in the AWHS continues to rise, from about 0600 until about 0900, when the energy available in the AWHS is at its peak for the day. This is expected as many water heater users are taking showers during this time in the morning as they get ready to go about their day. The available energy slowly levels off until about 1600, when a second but lower peak is seen in the evening time, corresponding to when customers return home from work or their respective activities of the day. 


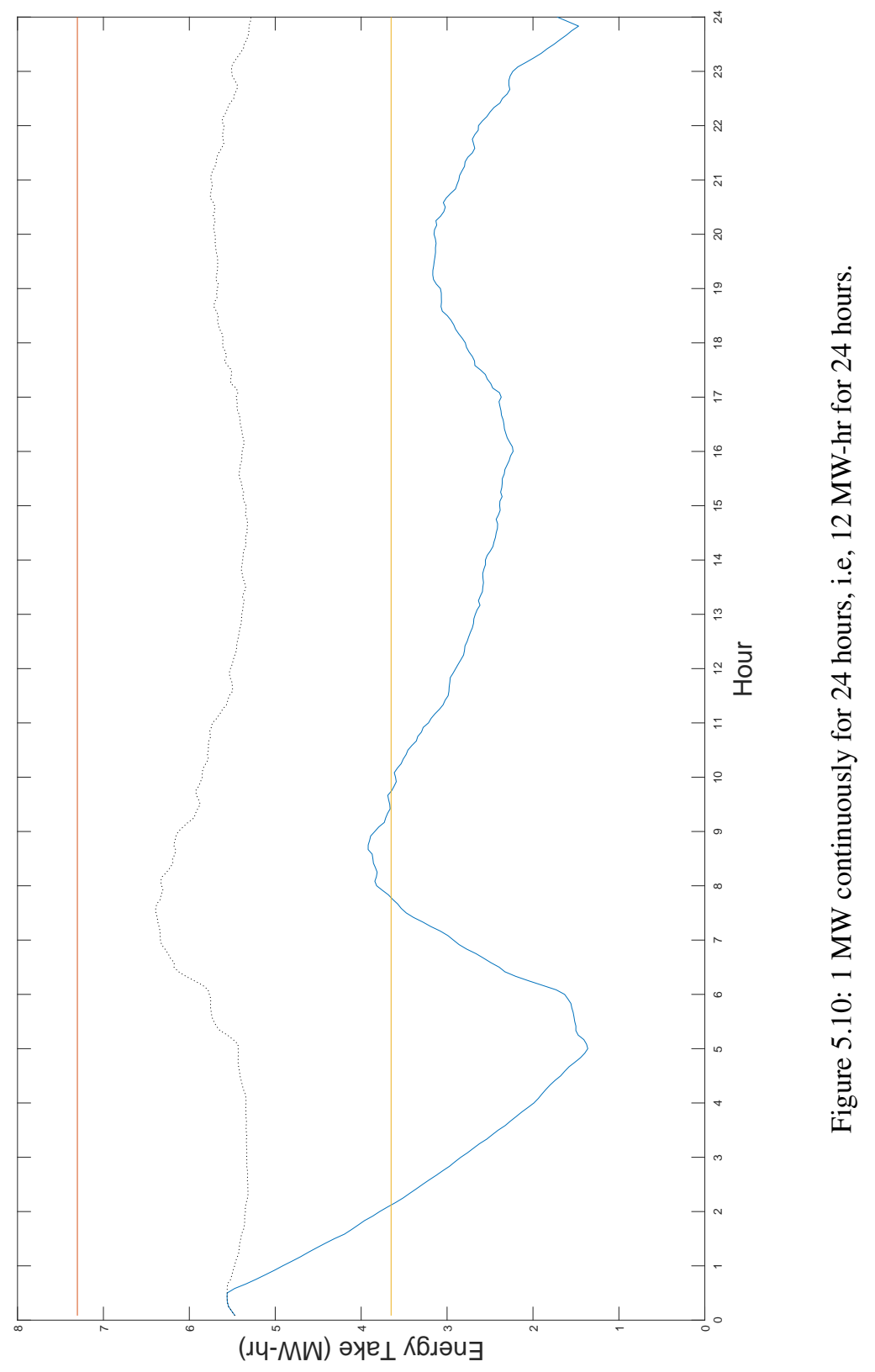


In Figure 5.11, we simulate several separate hourly dispatches, from 0300 to 1200, in an effort to understand the recovery times based on the time of dispatch. The recovery times are observed to be shorter for dispatches that occurred earlier in the morning than for those that occurred later in the morning. This observation is consistent with out expectations as more units will be brought online as people prepare and get ready for their day.

We simulated a weekend to see if there are any noticeable shift in patterns and we extend our hourly dispatches. Similarly, the later dispatches recover more quickly than earlier dispatches, even though in this case we conducted our dispatches through noon of a weekend. 


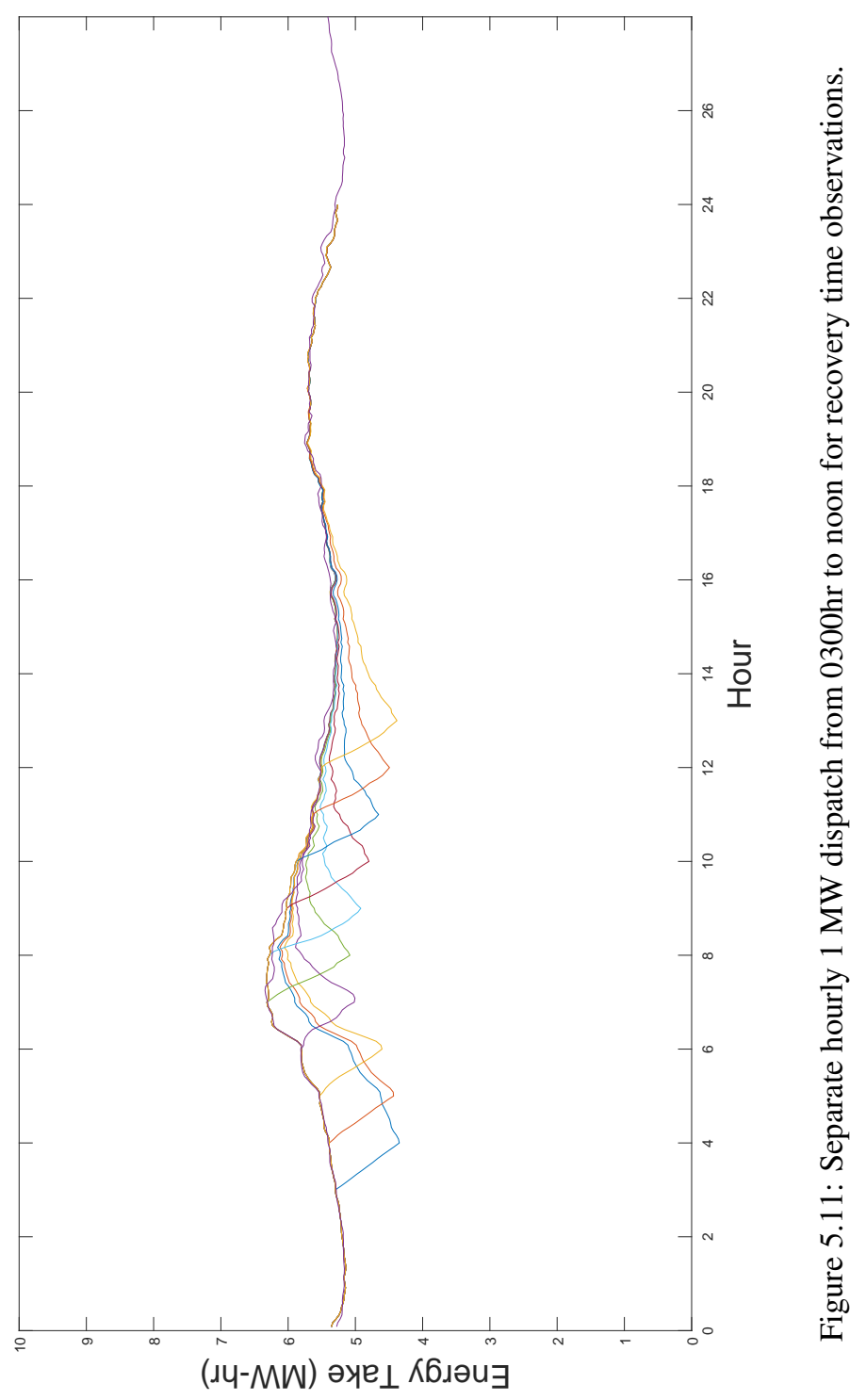




\begin{tabular}{|l|l|}
\hline $\begin{array}{l}\text { Distpatch } \\
\text { time }\end{array}$ & $\begin{array}{l}\text { Recovery times } \\
\text { in hours }\end{array}$ \\
\hline 3:00:00 & 7.81 \\
\hline $4: 00: 00$ & 7.88 \\
\hline 5:00:00 & 7.91 \\
\hline $6: 00: 00$ & 7.97 \\
\hline $7: 00: 00$ & 8.02 \\
\hline $8: 00: 00$ & 8.1 \\
\hline 9:00:00 & 8.12 \\
\hline 10:00:00 & 8.16 \\
\hline 11:00:00 & 8.19 \\
\hline 12:00:00 & 8.26 \\
\hline
\end{tabular}

Table 5.1: Dispatch recovery times from $0300 \mathrm{hr}$ to noon observed from figure 5.11.

The study of the recovery time of the AWHS is important when considering the opportunity costs of dispatching the AWHS. From Table 5.1, the conclusion of this study is that later dispatches take longer to recover than earlier dispatches. Our method requires that we continually anticipate future energy take data before the next dispatch takes place.

In Figure 5.12, we project the day-ahead's energy profile. The next day's forecast process is used to inform the AWHS on when to dispatch for revenue maximization. We discuss the forecasting process in the section that follows. 


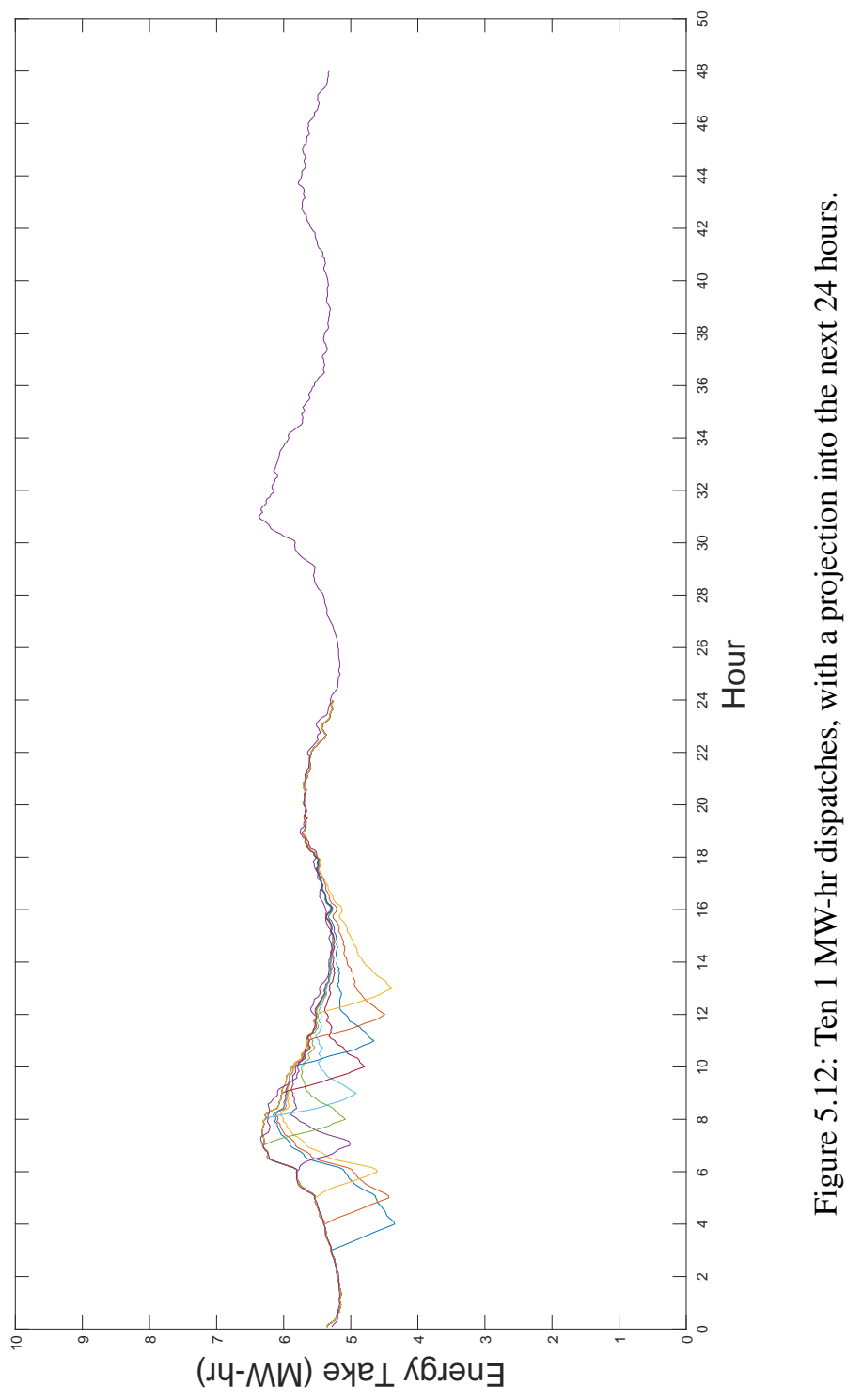




\subsection{Data Forecasting and Convergence}

\subsubsection{Energy Forecasting}

The optimization we developed uses future projections of two data sets, namely, the energy take of the AWHS and LMP market prices obtained from PJM. We do not forecast ambient temperature in this work, but instead, we use the National Oceanic and Atmospheric Administration (NOAA)'s publicly available forecast data.

We use LSTM to perform the forecasts in this work because traditional forecasting and regression models do not perform very well with large datasets. In addition, LSTM is capable of learning dependence of data based on what has been observed in the past, and applying this new observation to future predictions.

Convergence is achieved when the forecasted data approach a pre-defined limit, L. To evaluate the effectiveness of our forecasted data, we develop a convergence test algorithm that compares projected data to historical data. We use historical data for our test because testing on historical data would avoid over-training of our dataset. During the convergence test, we check to ensure that the projected data are approaching or within our limit. We arbitrarily chose a limit, $\mathbf{L}$ of $5 \%$ of the historical data. We test for convergence by updating the number of features, the root mean square error boundaries, or the number of training data after every iteration until the forecasted data is within $\mathbf{L}$.

We begin by examining a full year's worth of energy take data. In Figure 5.13, we observe three breaks in the energy take of the AWHS. The first and third are towards the end of the months of May and December, corresponding to Memorial day and Christmas 
day holidays respectively. We suspect the break in the middle of August to be a summer vacation break. 


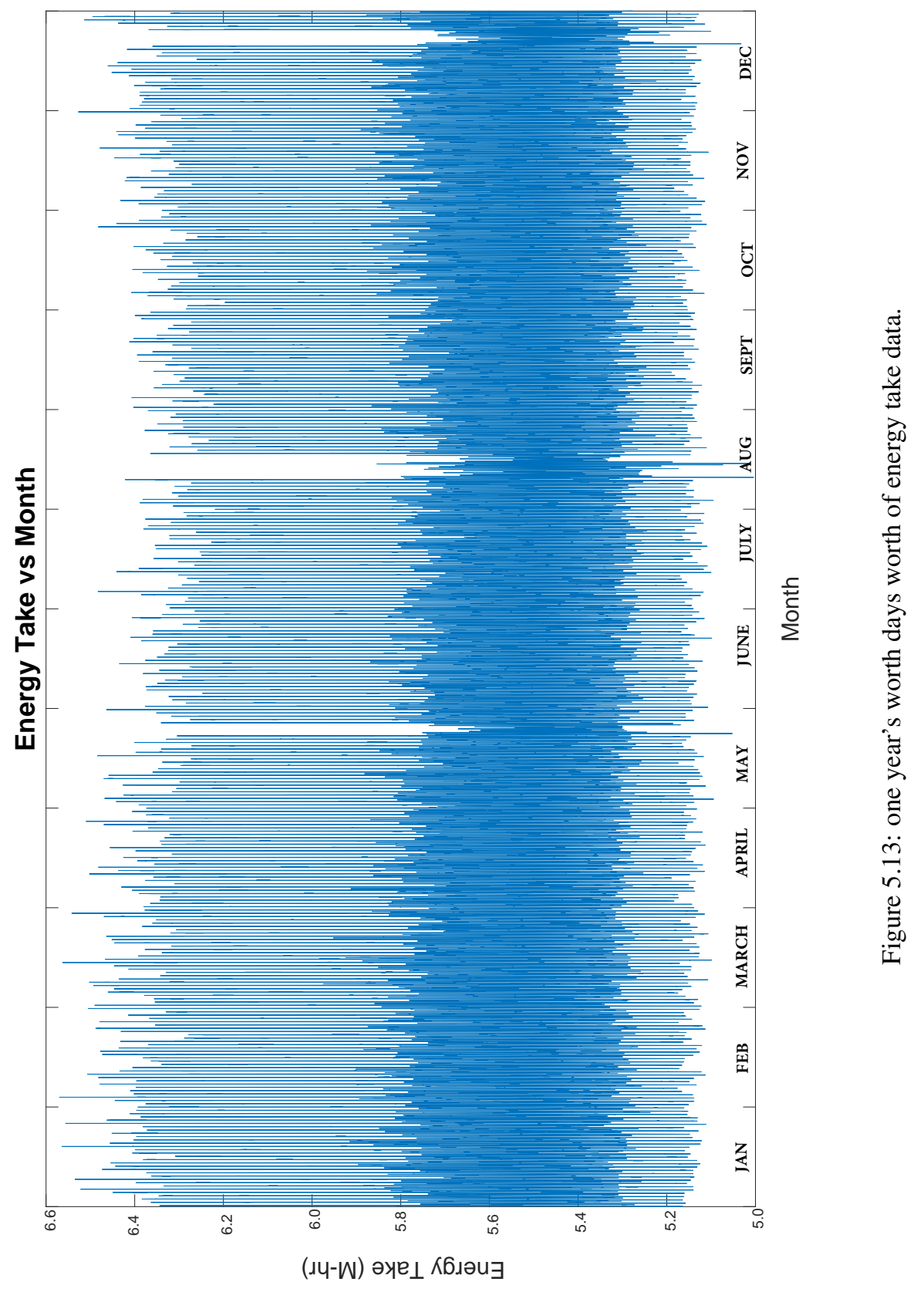


For our work, these two holidays were avoided when sampling our dataset. The vacation break in the month of August was also avoided. These data were avoided because the energy take on these days were significantly lower than on the other days of the year. In order to enhance the computational efficiency of our optimization, we chose a reduced, 28-day dataset in the month of July. We chose the month of July because there were no significant day to day changes in the energy take of the AWHS in the month of July, and we want to be able to simulate summer peak demand mitigation events that are typical of hot July days.

Figure 5.14 and Figure 5.15 shows the energy take for the first twenty eight days in July, and the first ten days in the month of July,respectively. The energy take during Weekends are not distinguished from week days. This may be because the AWHS is aggregating 10,000 water heaters, and at such a large scale, the difference in energy take between weekends and weekdays is negligible. The horizontal axis represents 288 -five minute intervals in each day. 


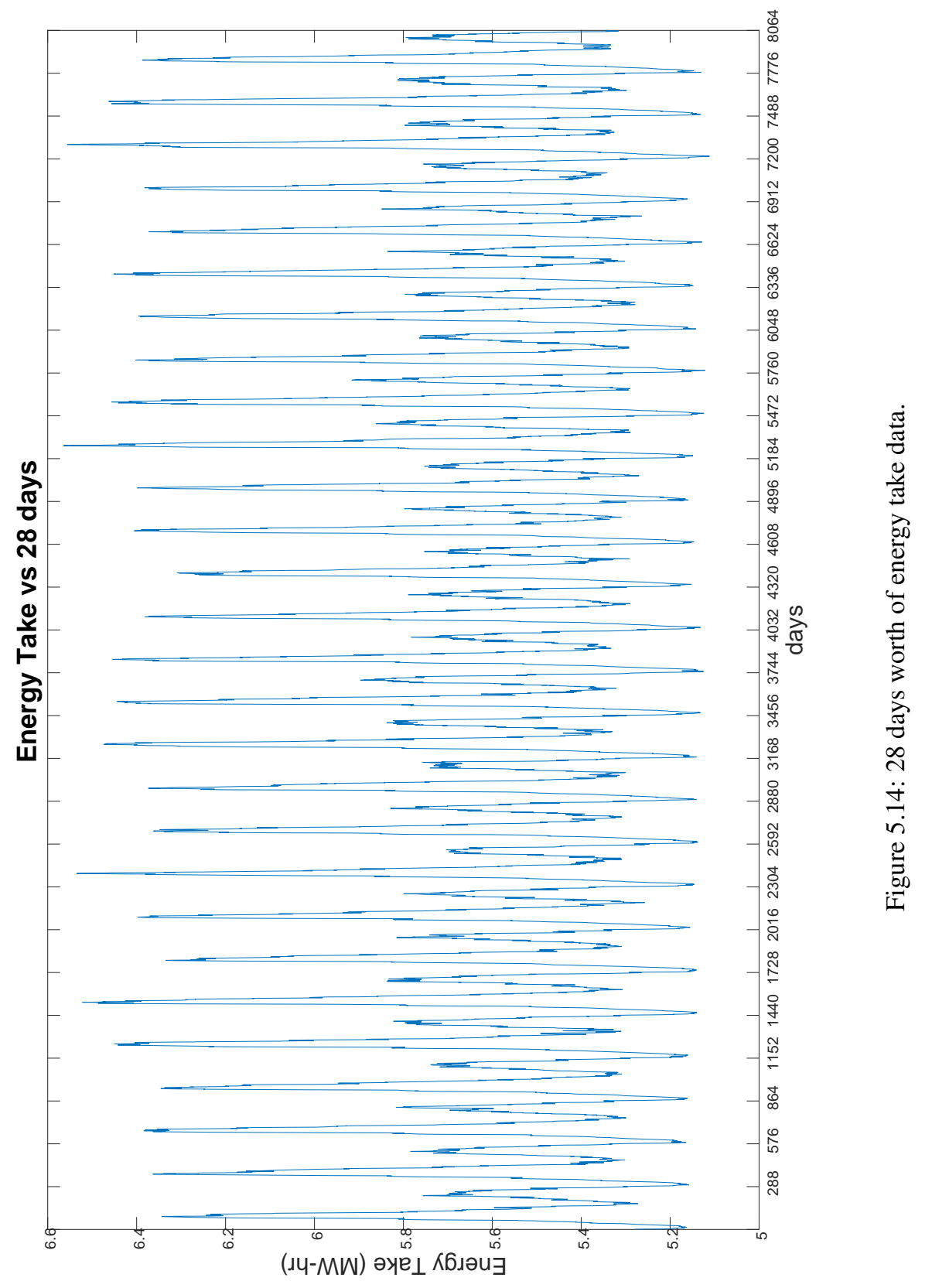


Because the shape of the energy take of the AWHS is similar for both weekdays and weekends, as we see in Figures 5.14 and 5.15, we have decided to treat the energy take of weekdays and weekends as the same in our optimization. In other words, no special scaling factor was applied for weekdays in comparison to weekends. 


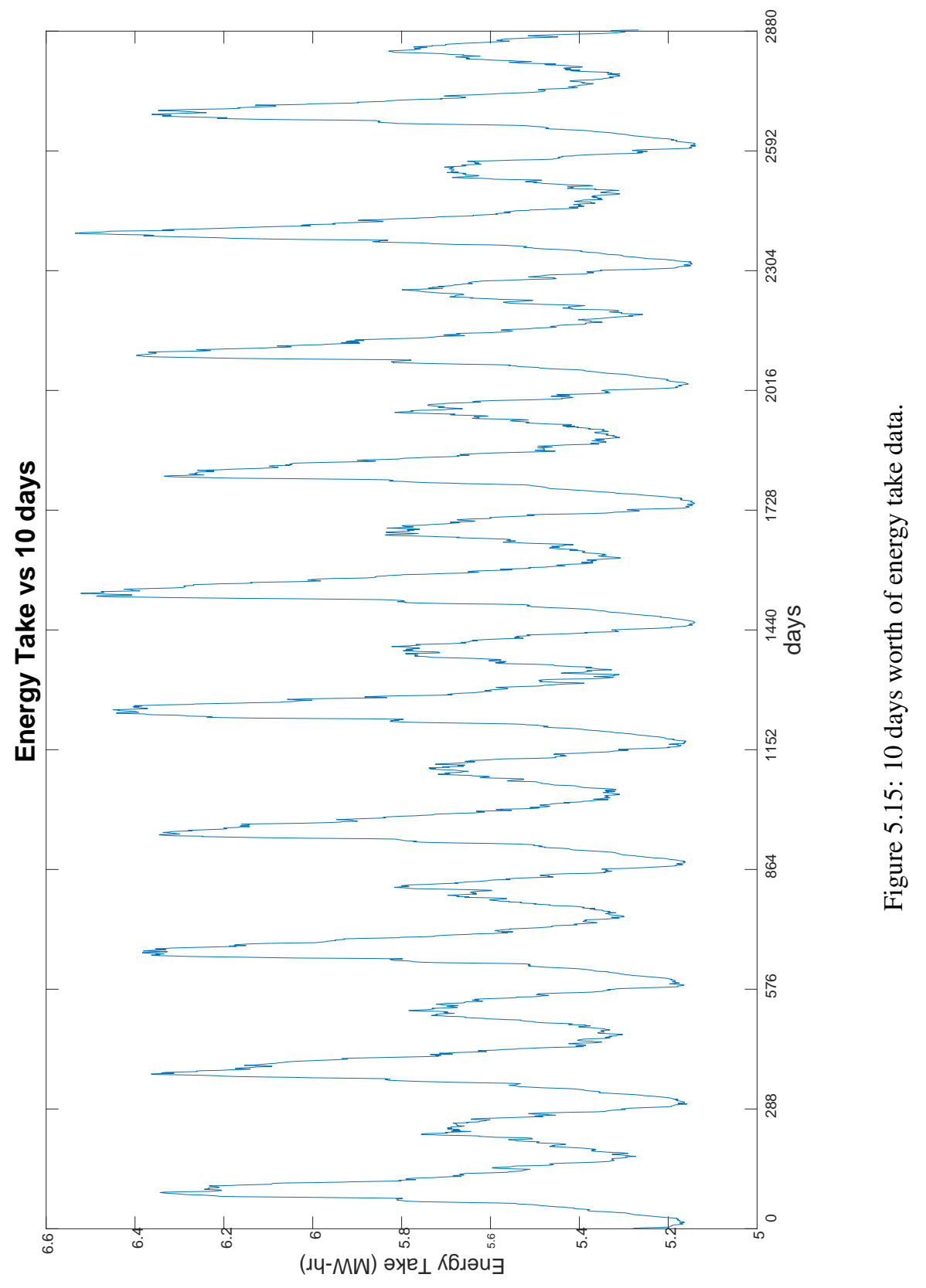


For energy take predictions, at first, we use two days, then four days, and then ten days worth of training data. More data were added to increase training efficiency and accuracy. Table 5.2 shows the summary of the statistics data for ten days and 28 days respectively.

\begin{tabular}{|l|l|l|}
\hline statistics & 10 day & 28 day \\
\hline min & $5.112 \mathrm{E}+06$ & $5.004 \mathrm{E}+06$ \\
\hline max & $6.564 \mathrm{E}+06$ & $6.570 \mathrm{E}+06$ \\
\hline mean & $5.602 \mathrm{E}+06$ & $5.582 \mathrm{E}+06$ \\
\hline median & $5.530 \mathrm{E}+06$ & $5.515 \mathrm{E}+06$ \\
\hline mode & $5.112 \mathrm{E}+06$ & $5.004 \mathrm{E}+06$ \\
\hline std & $3.253 \mathrm{E}+05$ & $3.088 \mathrm{E}+05$ \\
\hline range & $1.452 \mathrm{E}+06$ & $1.566 \mathrm{E}+06$ \\
\hline
\end{tabular}

Table 5.2: Summary statistics for ten days and 28 days Energy take (units in W-hr).

Upon close examination of both the ten days and 28 days Energy take values, the range increases as the number of days increases, meaning that there is more variation in the energy take over time. As expected, the standard deviation decreases, indicating that as we increase the data, most of the energy take values are closer to the mean.

In Figure 5.16, we start with two days worth of data, from July 1 to July 2. In this case, we attempt to forecast 12 hours worth of data (the second half of day 2) using only the prior 36 hours worth of learning data. A visual inspection of the forecasted data confirms that convergence is not achieved. Either more data are needed, or additional iterations are needed for the training model to achieve convergence. In other words, $25 \%$ of the time interval are forecast(test) after training on $75 \%$ of the dataset over 36 hours. 


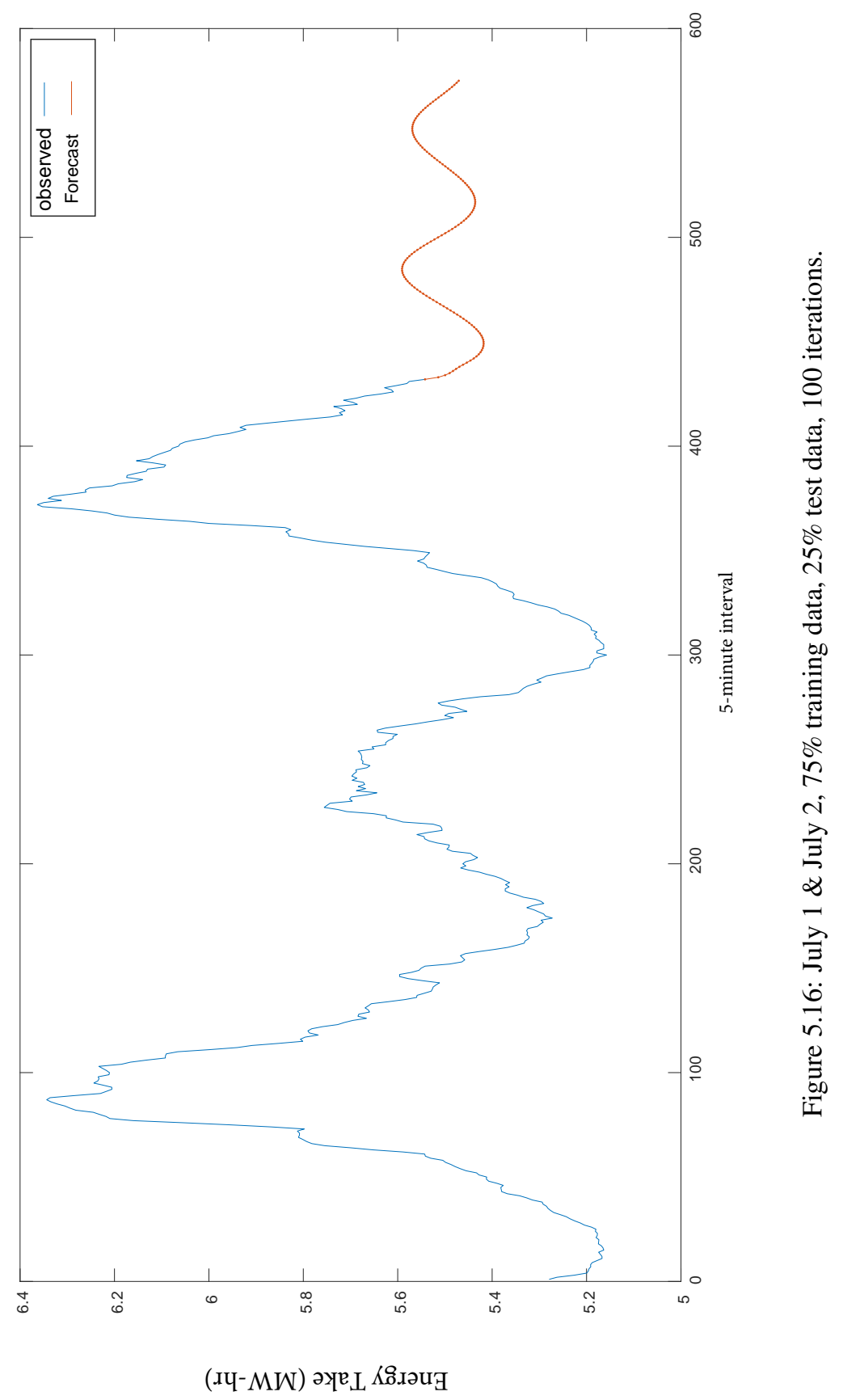


The plot in Figure 5.17 represents the same time period as the one in Figure 5.16, but with twice the number of iterations. For each batch of training, the weighted averages used in the LSTM algorithms are updated once. By doubling the amount of iterations, the weighted averages get twice the amount of updates for each pass through, resulting in better forecasted data, but requiring more computer memory and time. 


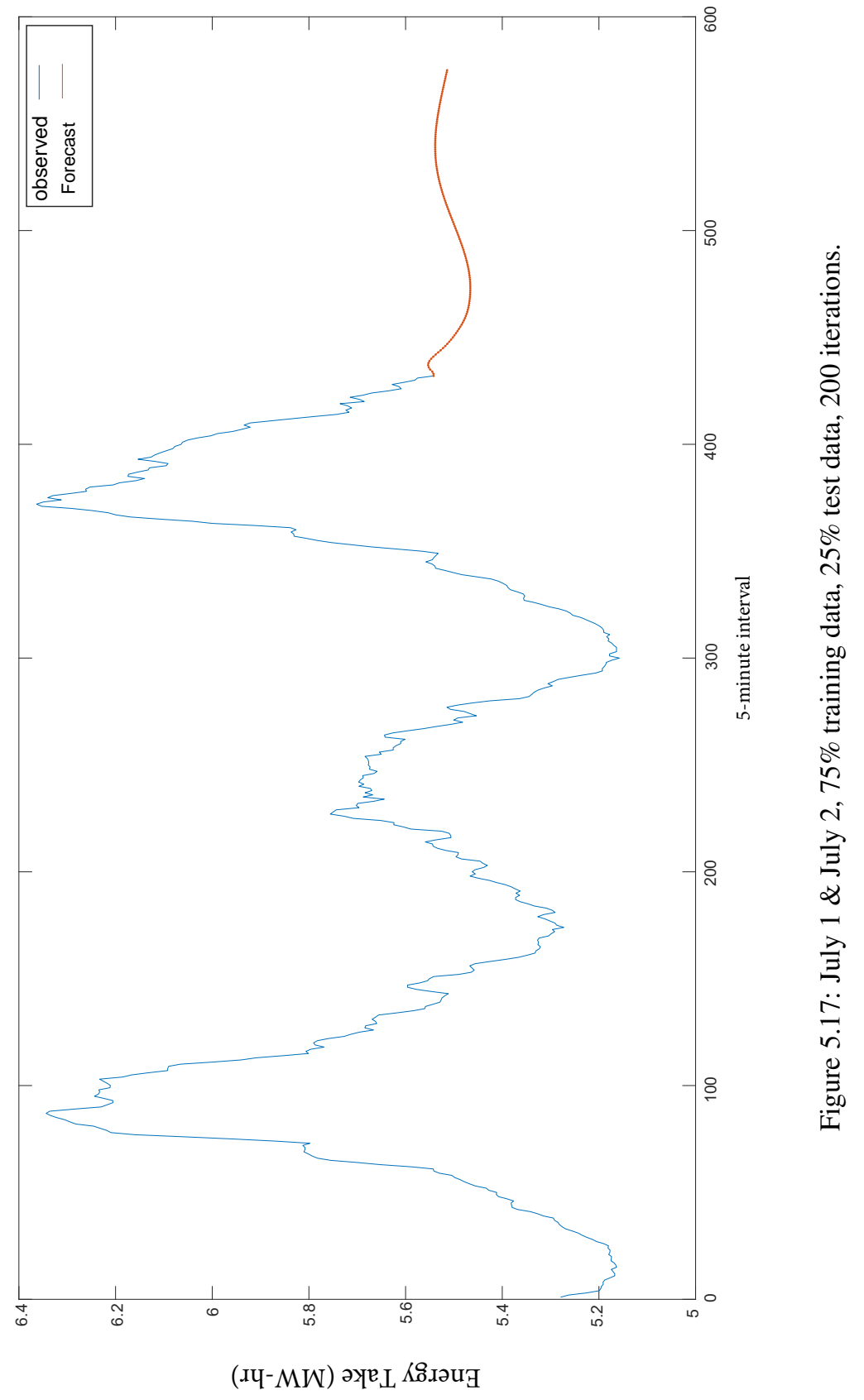


In Figure 5.18, we increase the number of training data. Here we have four days of historical data. We increase the amount of training data because there is an upper threshold after which, increasing the number of RNN iterations in the LSTM algorithm does not yield any additional benefits in terms of convergence. Increasing the number of training data helps us achieve convergence faster and avoids overtraining smaller datasets. 


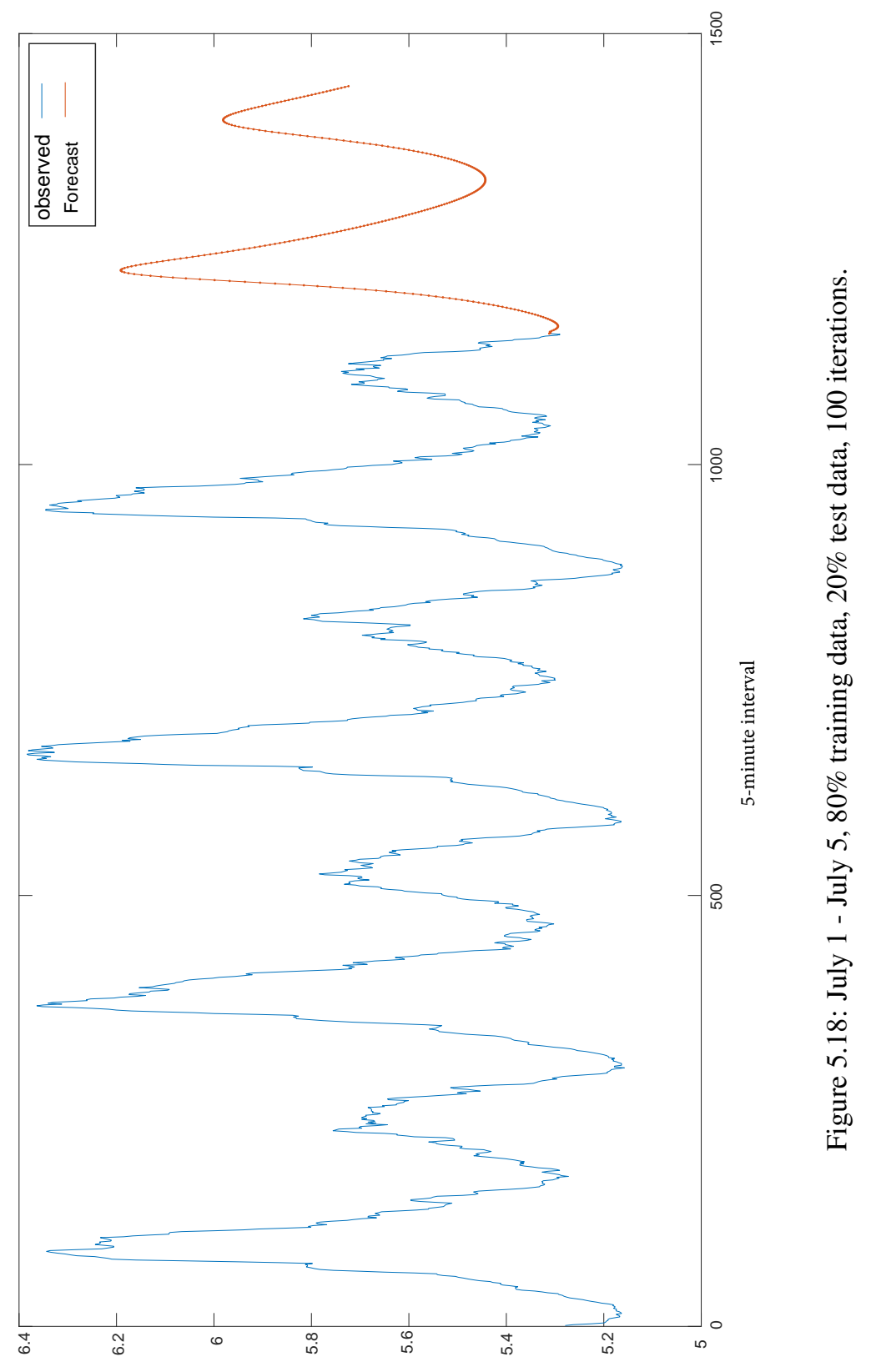

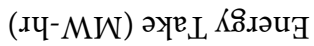


In Fig 5.19, we use ten days of historical data. The result is that a form of convergence begins to appear. We show only the 11th day date below, against the backdrop of day one's energy take data. 


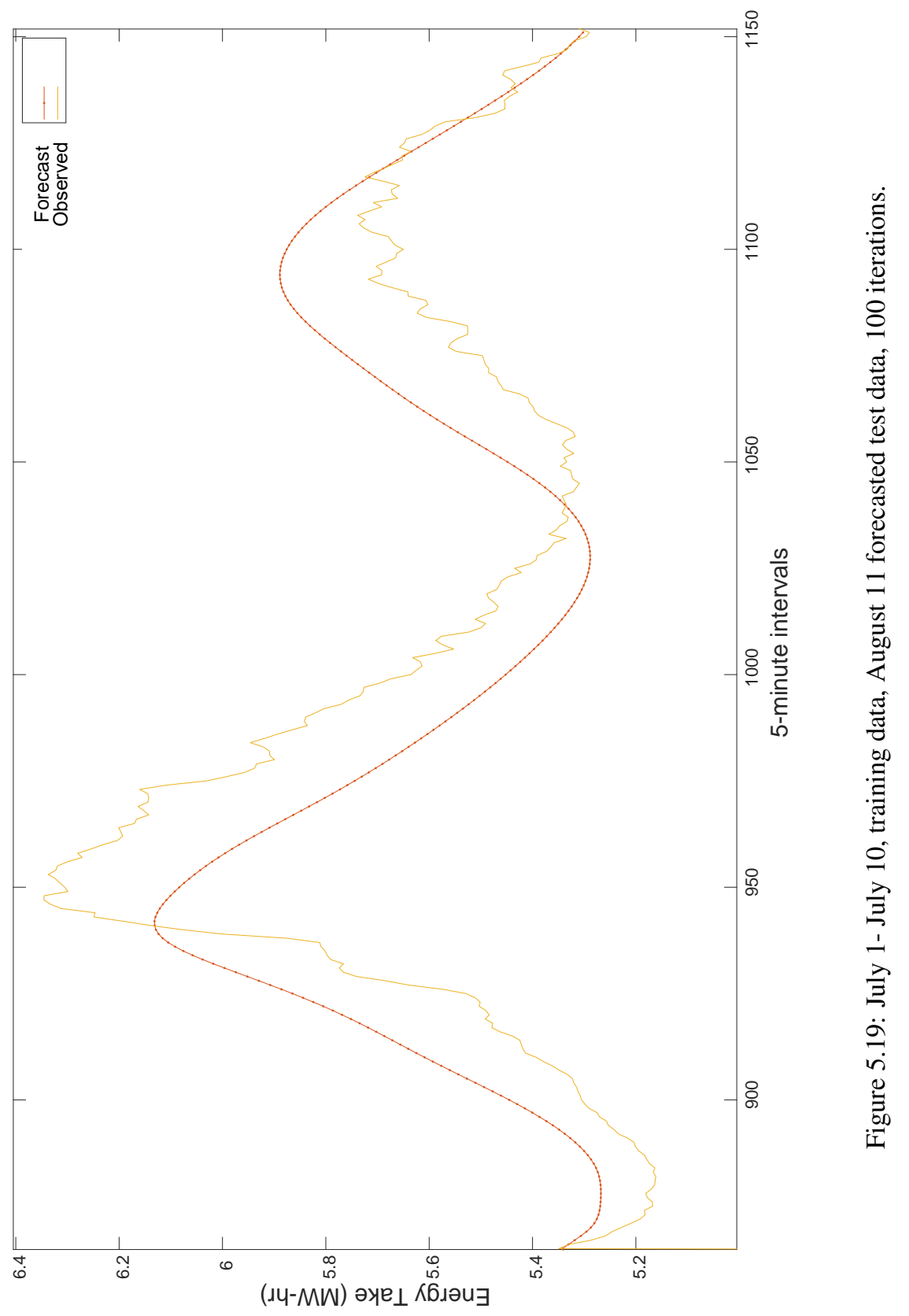


Our method considers the energy take for a given day's worth of data, which is then time-shifted on a moving five-minute average. Long Short Time Memory (LSTM) is applied to help with anticipating future data. Depending on the time of forecast, as we project further out into the future, the forecasted data begins to diverge from the historical data. In Figure 5.20, we iterate the LSTM algorithm 200 times, using 10 days worth of data. As expected, the closer we are to the time of simulation, time $t_{0}$, the more accurate our future projections will become. 


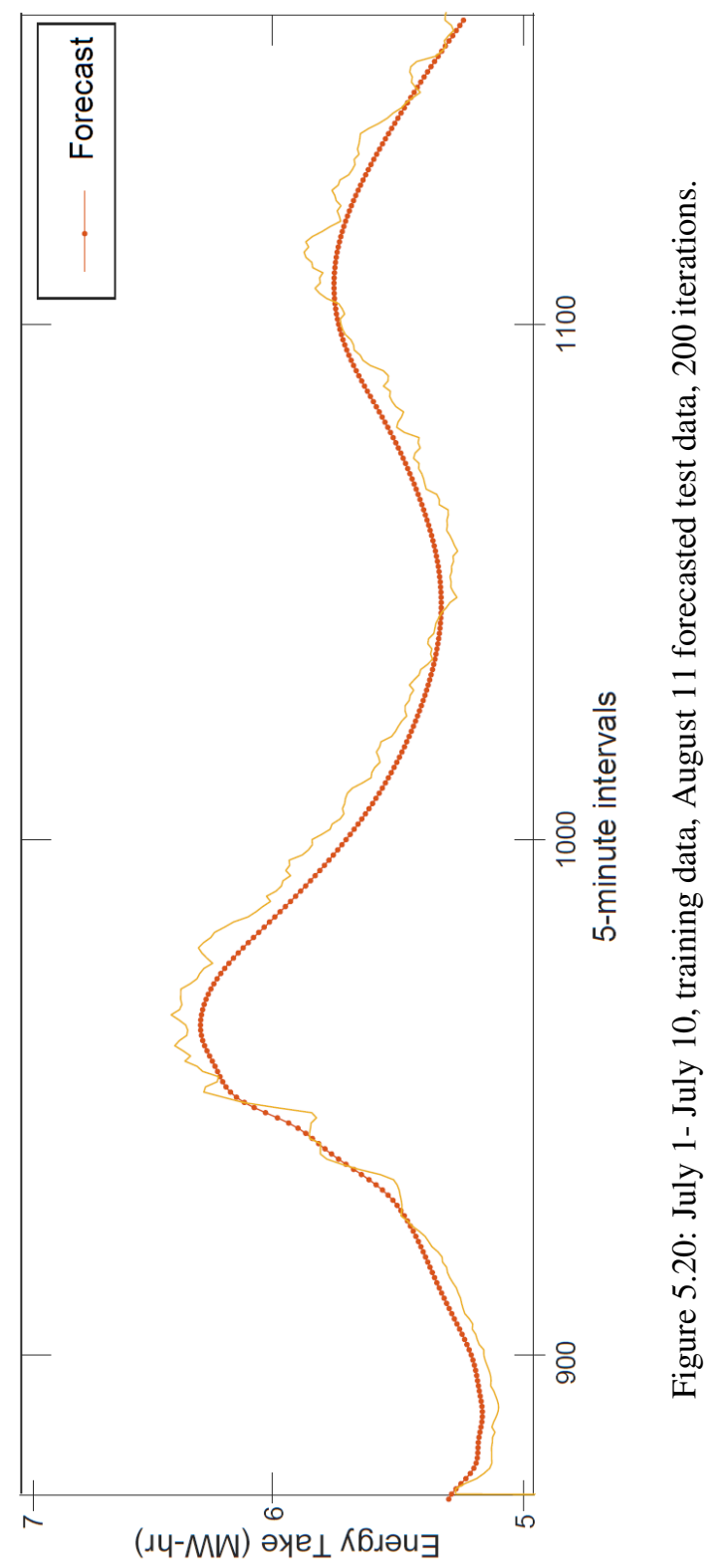


These results show that we can improve the accuracy of our forecast in one of two ways. First, by either increasing the number of iterations, where an iteration is one loop through the entire training data set, until a threshold is reached where increasing the number of iterations has no impact on the training. Another way to improve accuracy is by increasing using more training data sets. In Fig 5.19 and Figure 5.20, increasing the number of iterations from 100 to 200 help to quickly achieve convergence. These results were heuristically obtained and can vary from dataset to dataset or time frame to time frame. In this case, we have only observed datasets from the first few days of the month of August.

We culminate our forecasting exercise with Figure 5.21, which shows our convergence test, where the Root Mean Square Error (RMSE) is calculated as 0.003 MW-hr or 2.993 KW-hr from Figure 5.21. Convergence is achieved and the RMSE is shown to be less than five percent. Our minimum Energy take is three times an order of magnitude (MW-hr) greater than the RMSE. In this instance the RNN observations passes the convergence test. 


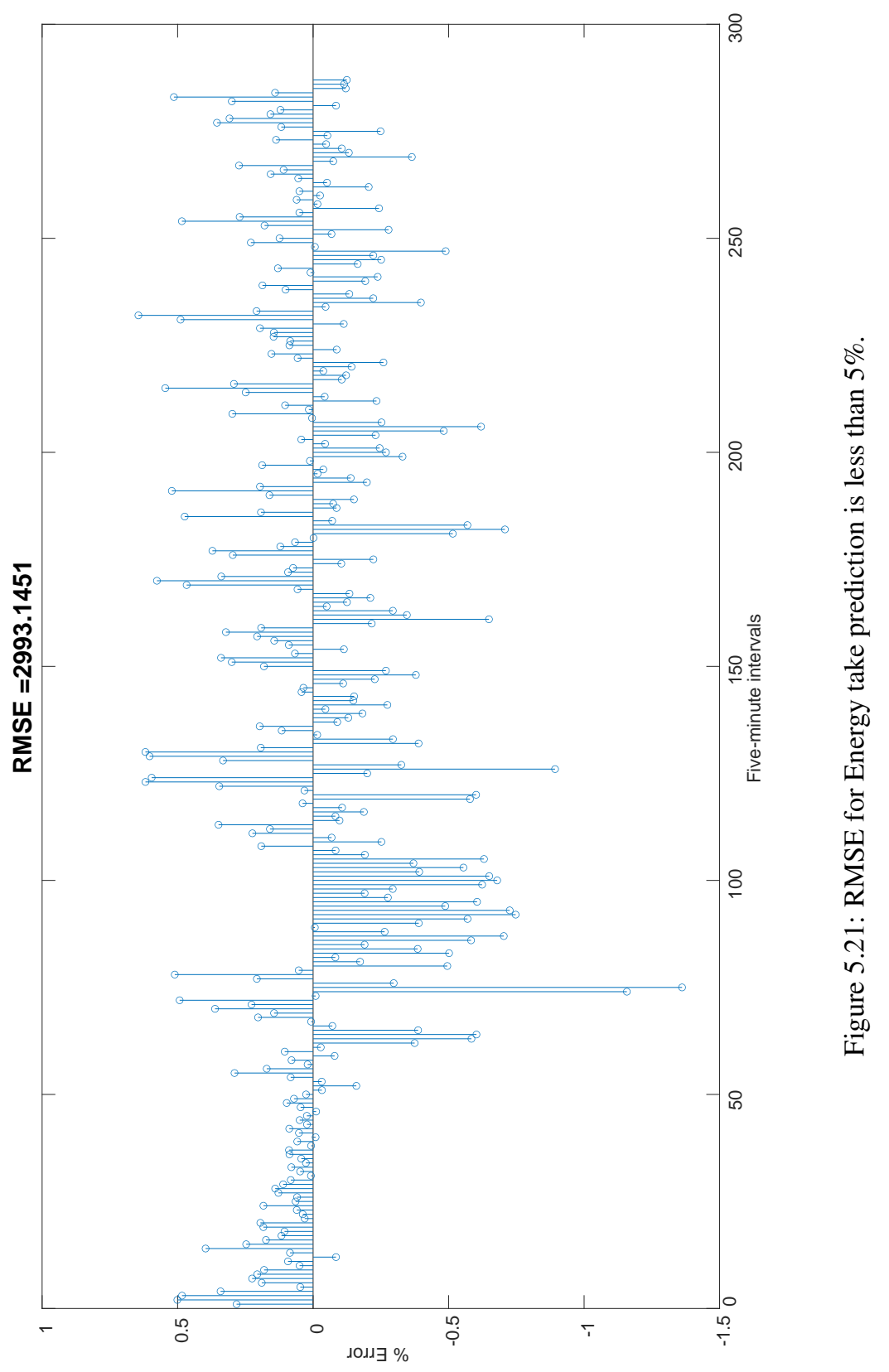




\subsubsection{Price Forecasting}

LSTM is used to forecast energy prices. This is of particular importance because the pricing structure used for peak demand mitigation is a variation of that of the energy price, with a multiplier applied during peak demand hours. We pull our data from PJM's LMP repository. Figure 5.22 shows three weeks of LMP market price data. The vertical axis represent the energy price in U.S dollars. There are two peaks in the daily energy prices, corresponding to the AM and PM energy demand cycles. 


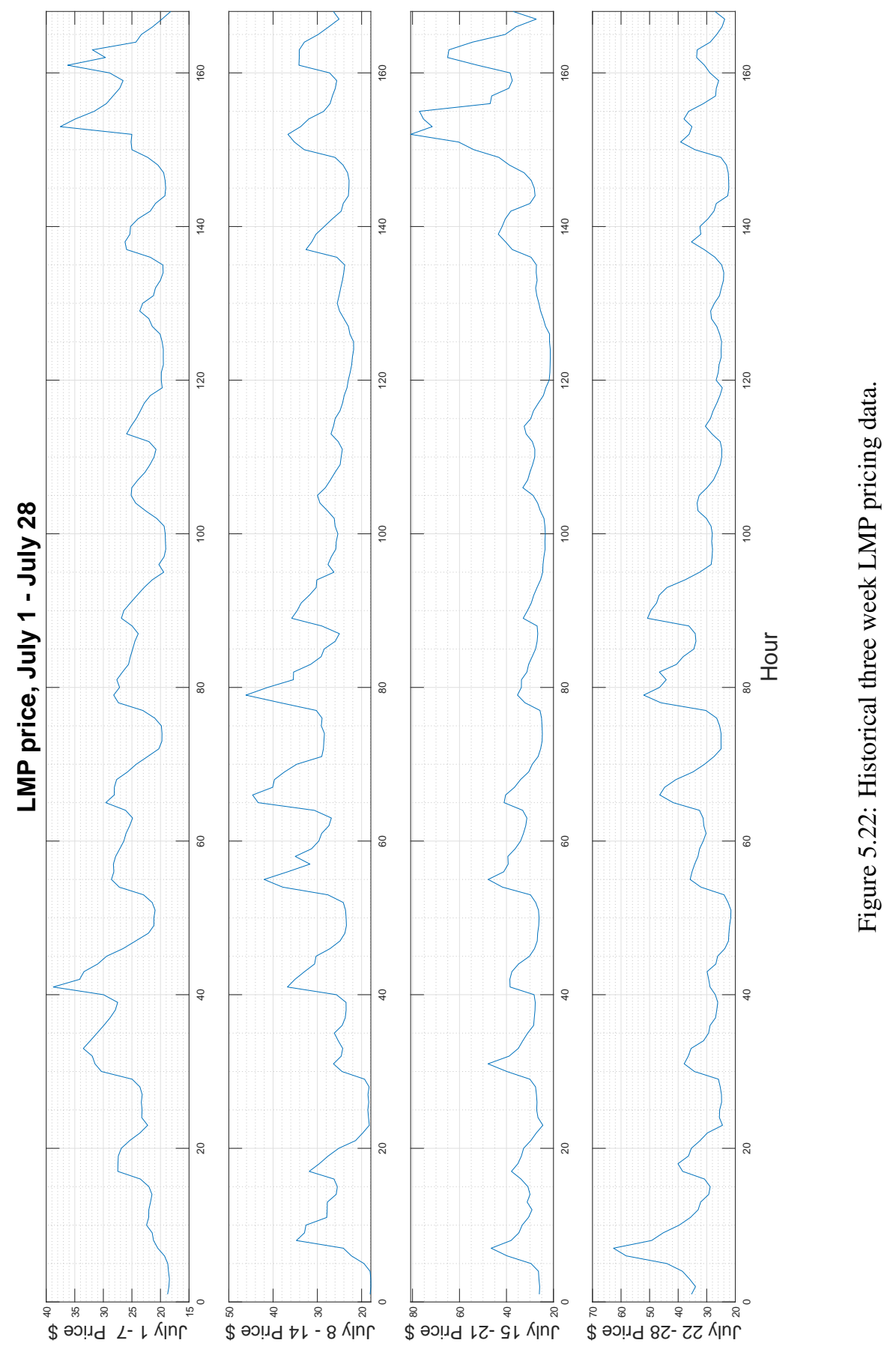


Table 5.3 is a summary of the LMP price data. We notice that the maximum LMP price occurs in week three, while the minimum LMP price was observed in the second week. In the former, prices had the highest swings with the highest rage of $\$ 59.44$.

\begin{tabular}{|l|l|l|l|l|}
\hline Statistics & July 1- 7 & July 8 14 & July 15 - 21 & July 22-28 \\
\hline min & 18.19 & 17.91 & 21.35 & 21.52 \\
\hline $\max$ & 38.77 & 46.14 & 80.79 & 62.66 \\
\hline mean & 24.33 & 27.69 & 32.96 & 31.42 \\
\hline median & 23.62 & 26.37 & 29.72 & 29.34 \\
\hline mode & 21.45 & 22.99 & 25.48 & 25.47 \\
\hline std & 4.213 & 5.54 & 10.42 & 7.529 \\
\hline range & 20.58 & 28.23 & 59.44 & 41.14 \\
\hline
\end{tabular}

Table 5.3: LMP prices in US \$.

We forecast future energy prices in Figure 5.23. Similar to the forecast conducted for energy take, only the first few hours of LMP price correlate with expected historicals. The price begins to deviate from observed historical price data the further out we project. 


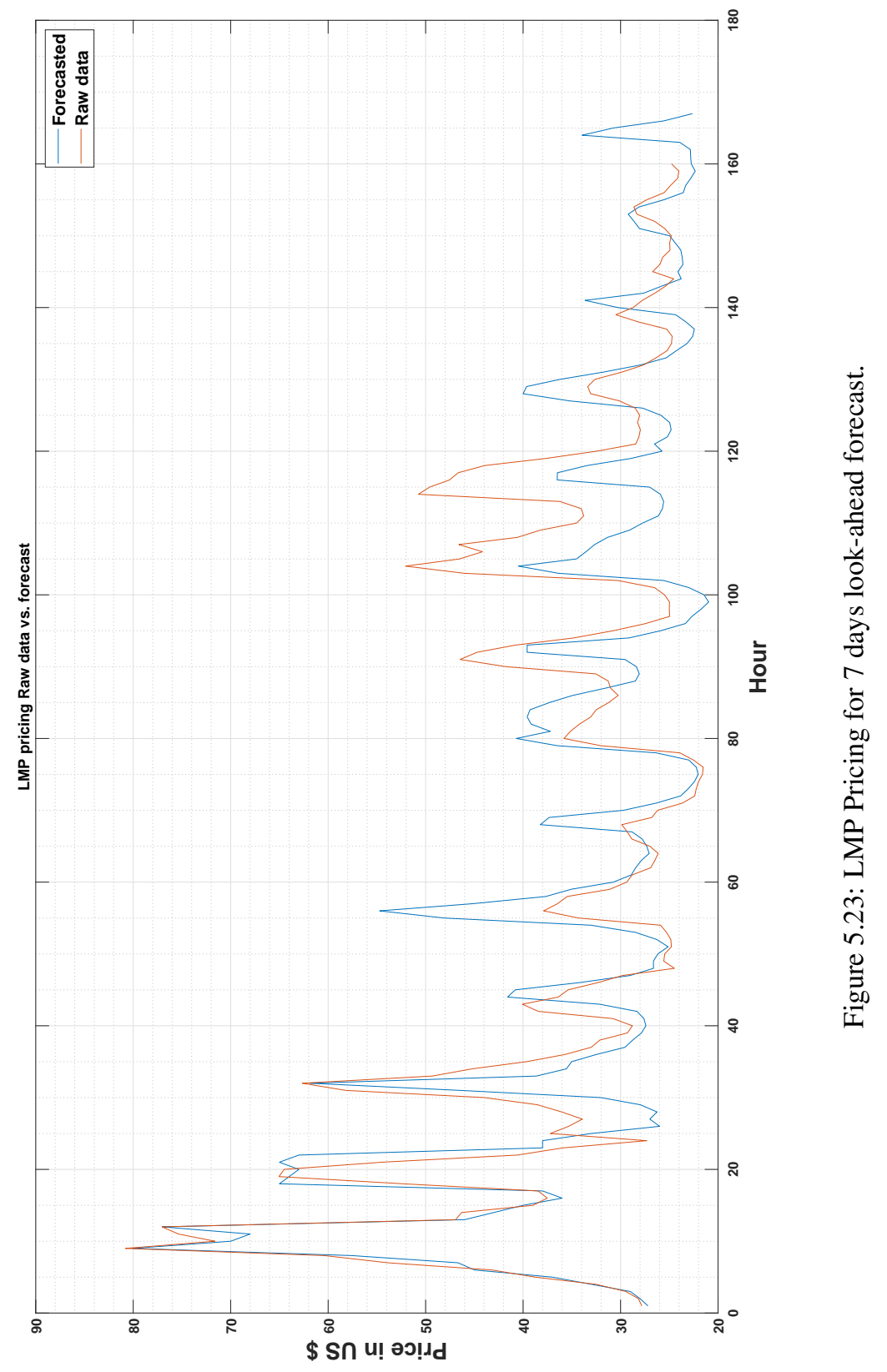


In Figure 5.24, we show that the RMSE for LMP is $\$ 0.172$, less than $5 \%$ of the prices in figure 5.23 when iterating at least 100 times,and therefore it passed our convergence test. The RMSE gets larger as time increases. 


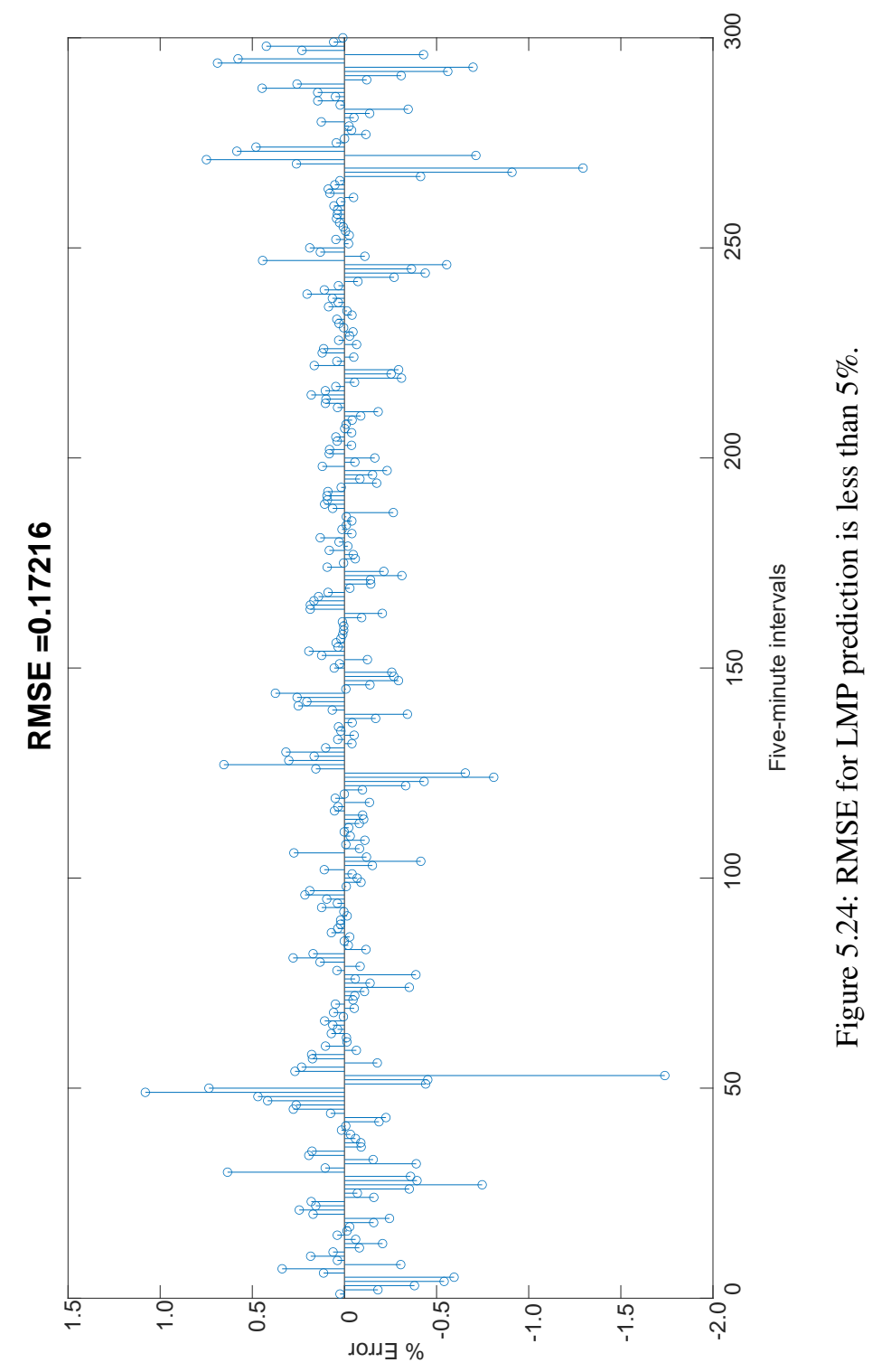




\subsubsection{Weather Forecasting}

Forecasted temperature data are required for peak demand mitigation. The weather data for the Portland Metropolitan area are retrieved from the National Oceanic and Atmospheric Administration (NOAA). NOAA has well-established means for forecasting temperatures, so we do not attempt to do so our selves.

We use NOAA's historical data from the year 2012 because it is one of the years with the highest recorded peak demand events. Figure 5.25 shows that there were ten sub-freezing days in 2012 for which peak demand mitigation was required. We examined the hottest days of 2012 . 


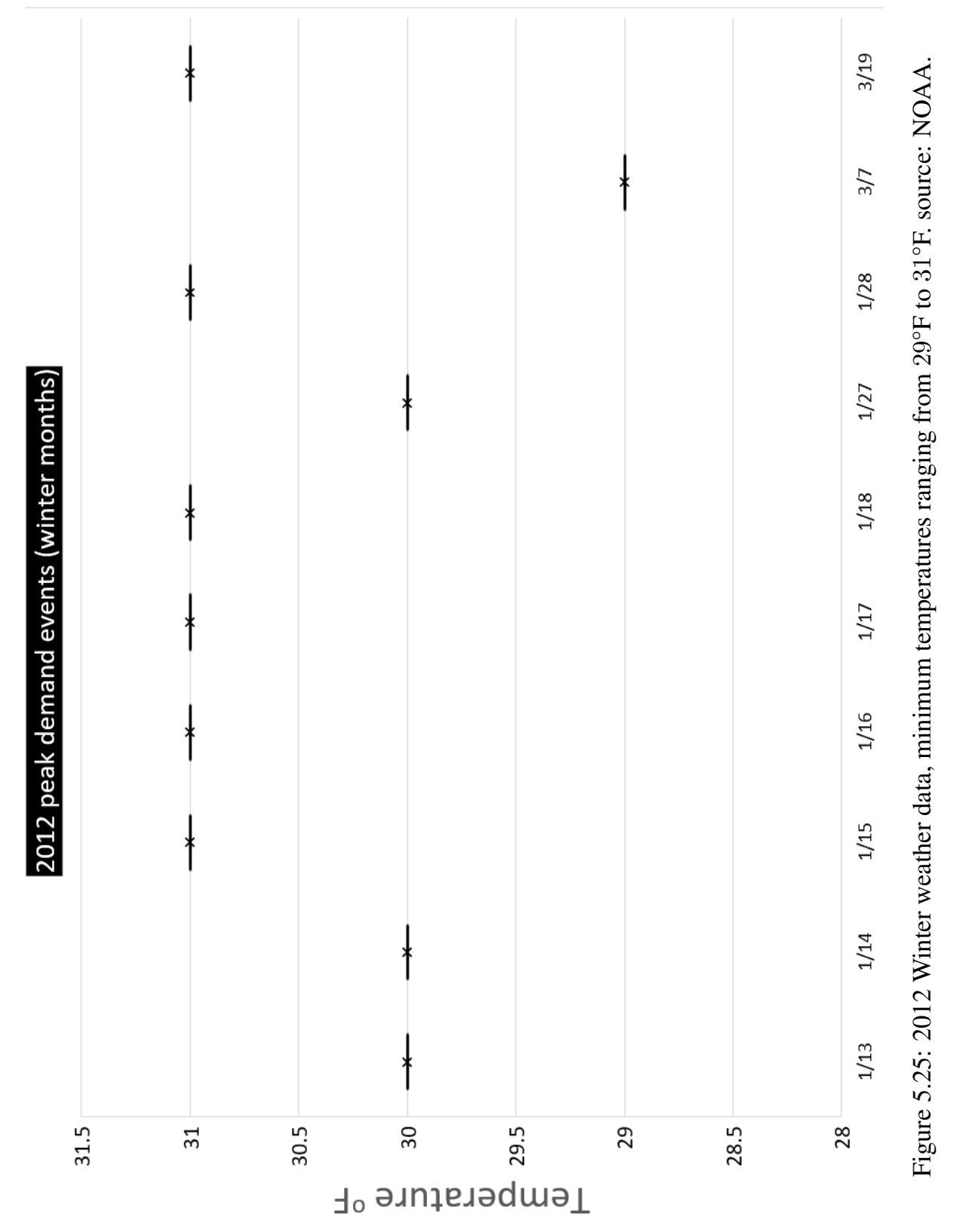


Figure 5.26 shows only seven days in 2012 with temperatures above 90 degrees Fahrenheit. For our research, new weather data is not predicted. We use existing data already provided by NOAA. 


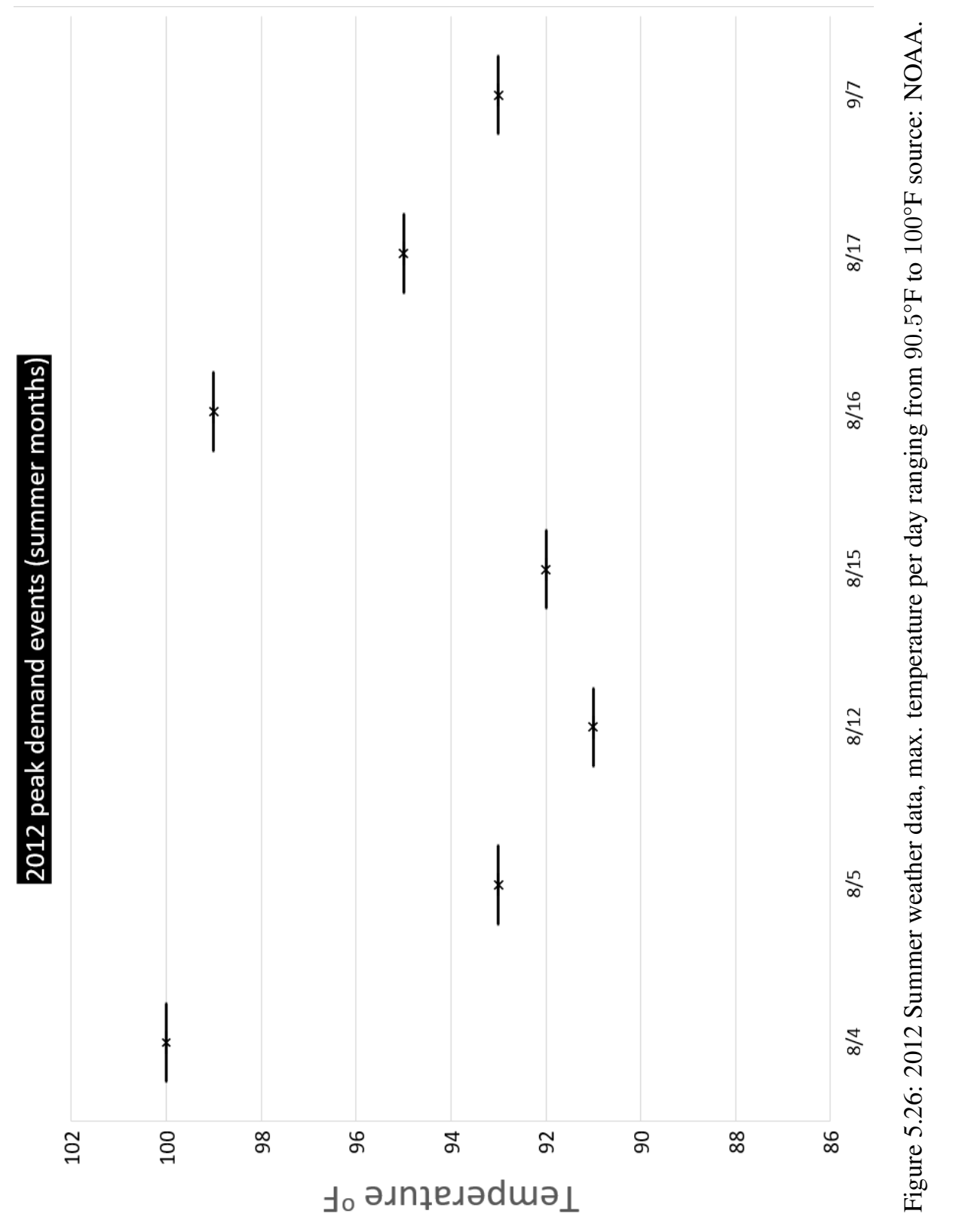


In total, for the year 2012, there were only seventeen days for which peak demand mitigation was requested. In fact, for the decade between 2010 and 2019, no year required peak demand for more than 20 days.

\subsection{Optimization of the AWHS}

In the following sections, we focus our attention on the major contribution of this research; the optimization of the AWHS for revenue maximization and the determination of the appropriate ratios to dispatch the aforementioned ancillary services.

The revenue to be generated by the AWHS can be maximized by determining the appropriate proportion of each of the three ancillary services to schedule. The constraints defined earlier in 4.2.2 define the dependence between power and energy of the dispatch service; frequency regulation, which in turn determines the number of units to dispatch. Peak demand mitigation, a reserve service, is implemented based on weather temperature forecast data, and on the applicable time periods. Frequency response, also a reserve service; is unique in that dispatches are rare and stochastic in nature.

\subsection{Stage 1 Optimization}

Prior to the first stage optimization, historical data are used to generate forecasts. In stage 1, the optimization routine anticipates future energy, ambient temperature, and market prices based on historical data. The three ancillary services are scheduled as reserve services with no dispatch whatsover. Upon convergence, the optimization algorithm shifts to the next 
five-minute block, repeats the optimization, and updates the projected allocations. This process is repeated until a full day's worth of projections (288 projections) are simulated. The algorithm then allocates the ancillary services for revenue maximization using the forecasted data, and assuming that all three services are reserve services.

In the following paragraphs, we explore the behavior of the AWHS in stage 1 by varying the prices of all three services. The optimization algorithm uses the data available to forecast an entire day's worth of energy take data. The top plot in the figures that follow represents the price of each service and the bottom plot represent the pre-dispatch optimized energy take profile.

\subsubsection{Case 1.1 - Limiting services}

We explore a scenario where we may need to limit the amount of energy take reserved for each service. In this case, we attempt to to verify our ability to set reserve limits in the AWHS. In this case, optimization has not been applied yet. We expect that the services will be capped at the upper limit specified. 


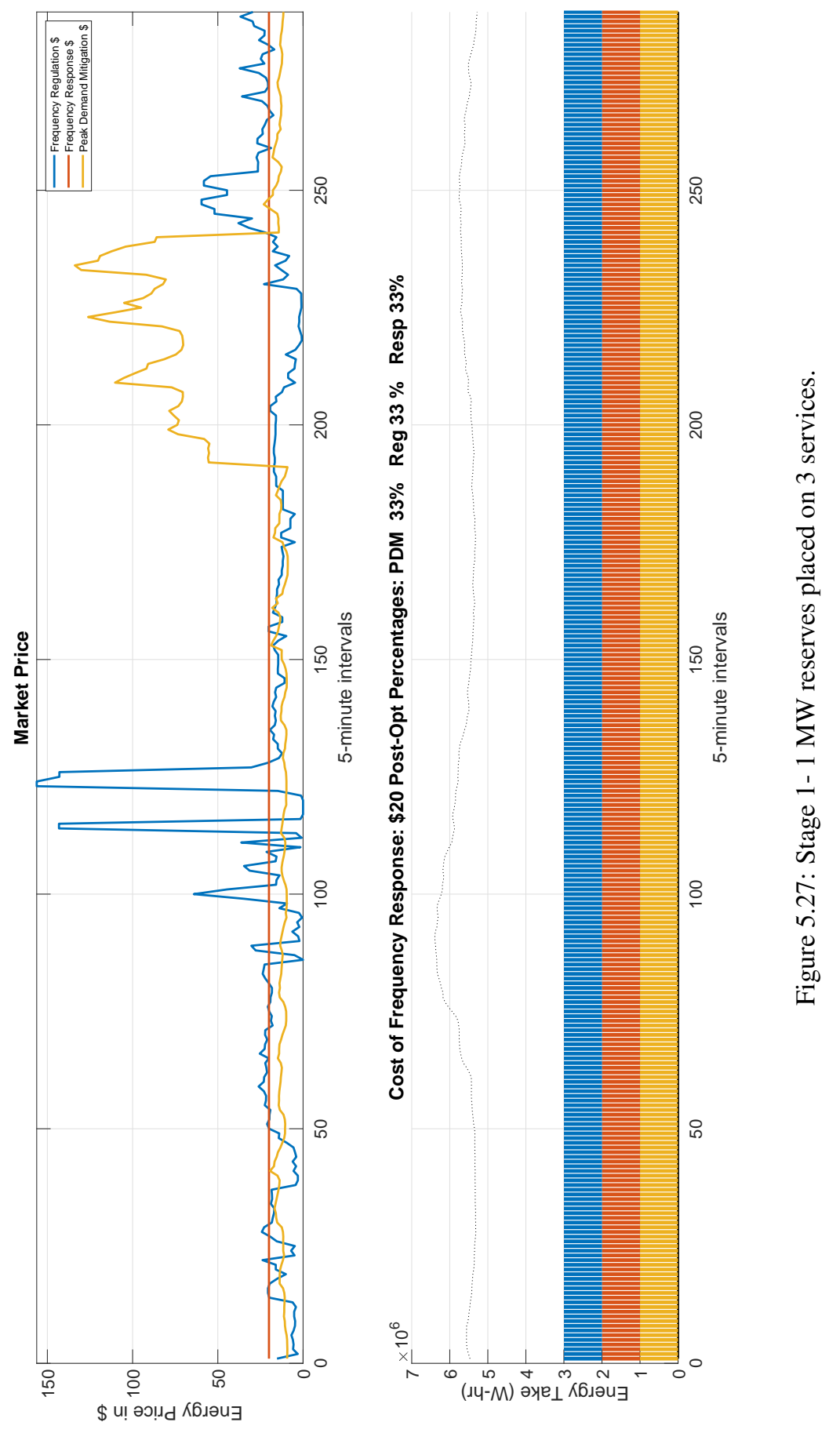


The results shown in Figure 5.27 is in line with our expectations, and they demonstrate our ability to reserve any of the three services in stage 1; the pre-dispatch stage. In Figure 5.27, we manually limit the amount of services reserved for for peak demand mitigation, frequency regulation and frequency response to $1 \mathrm{MW}-\mathrm{hr}$.

When called upon, the optimization algorithm will only dispatch $1 \mathrm{MW}-\mathrm{hr}$ of each service. As expected, the ratio allocated by the optimization is $33 \%$ for each service.

\subsubsection{Case 1.2 - Stage 1 Optimization}

We begin with an optimization in stage 1, when all services are treated as reserves, including frequency regulation. All services are maximised up to the maximum available energy take in the AWHS. We show the output of the stage 1 optimization in Figure 5.28 - 5.30. We chose this case to demonstrate how the optimization algorithm will behave on a day with no dispatches. As discussed earlier in section 5.5.3, peak demand is only required for a handful of days in the year, while frequency response is stochastically requested. The price

of frequency response was found to be very sensitive. We tested the sensitivity by varying the price between $\$ 20, \$ 25$ and $\$ 40$ and we noted that the percentages allocated to frequency response changed between $51 \%, 67 \%$, and $77 \%$ respectively. 


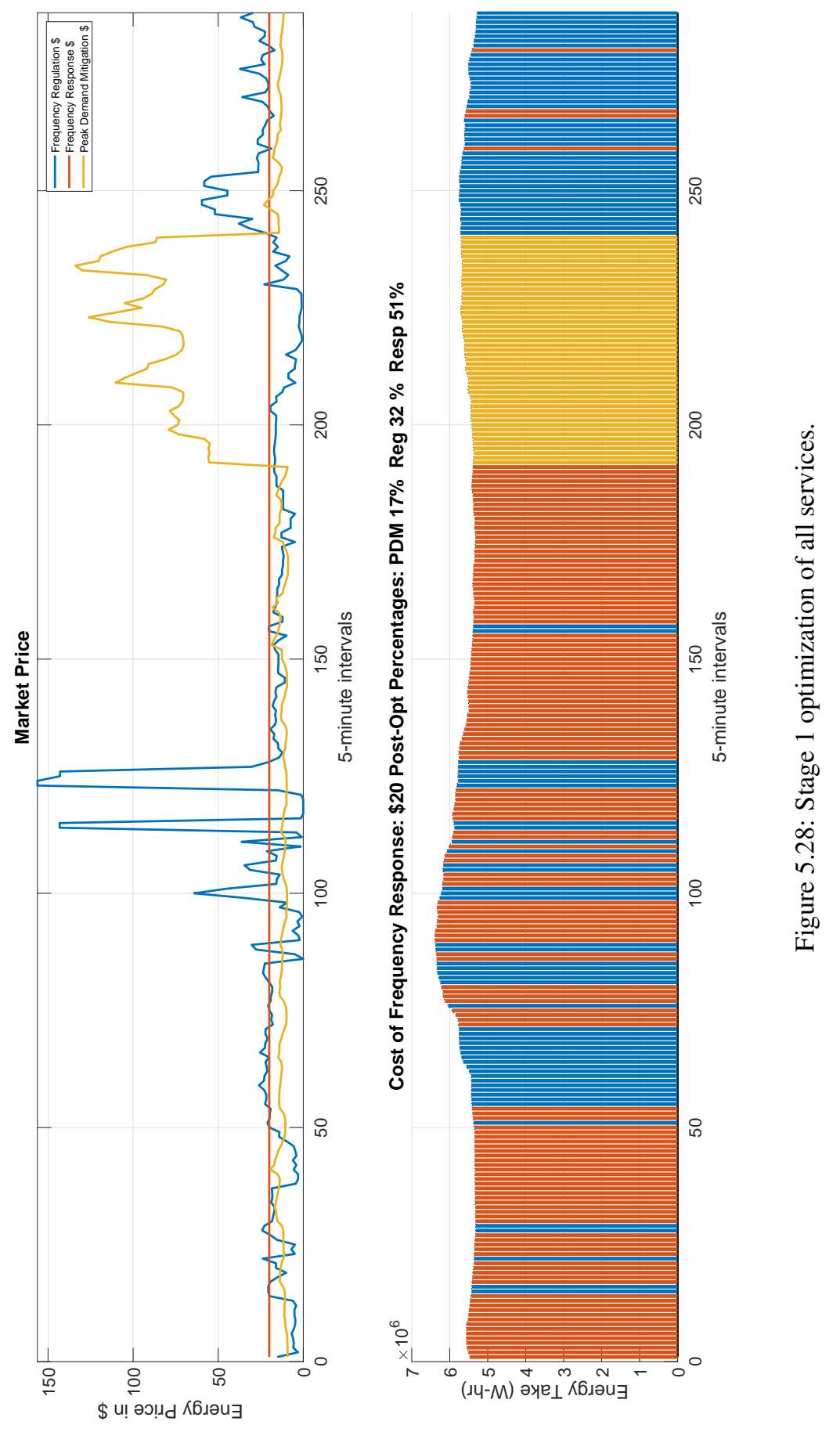




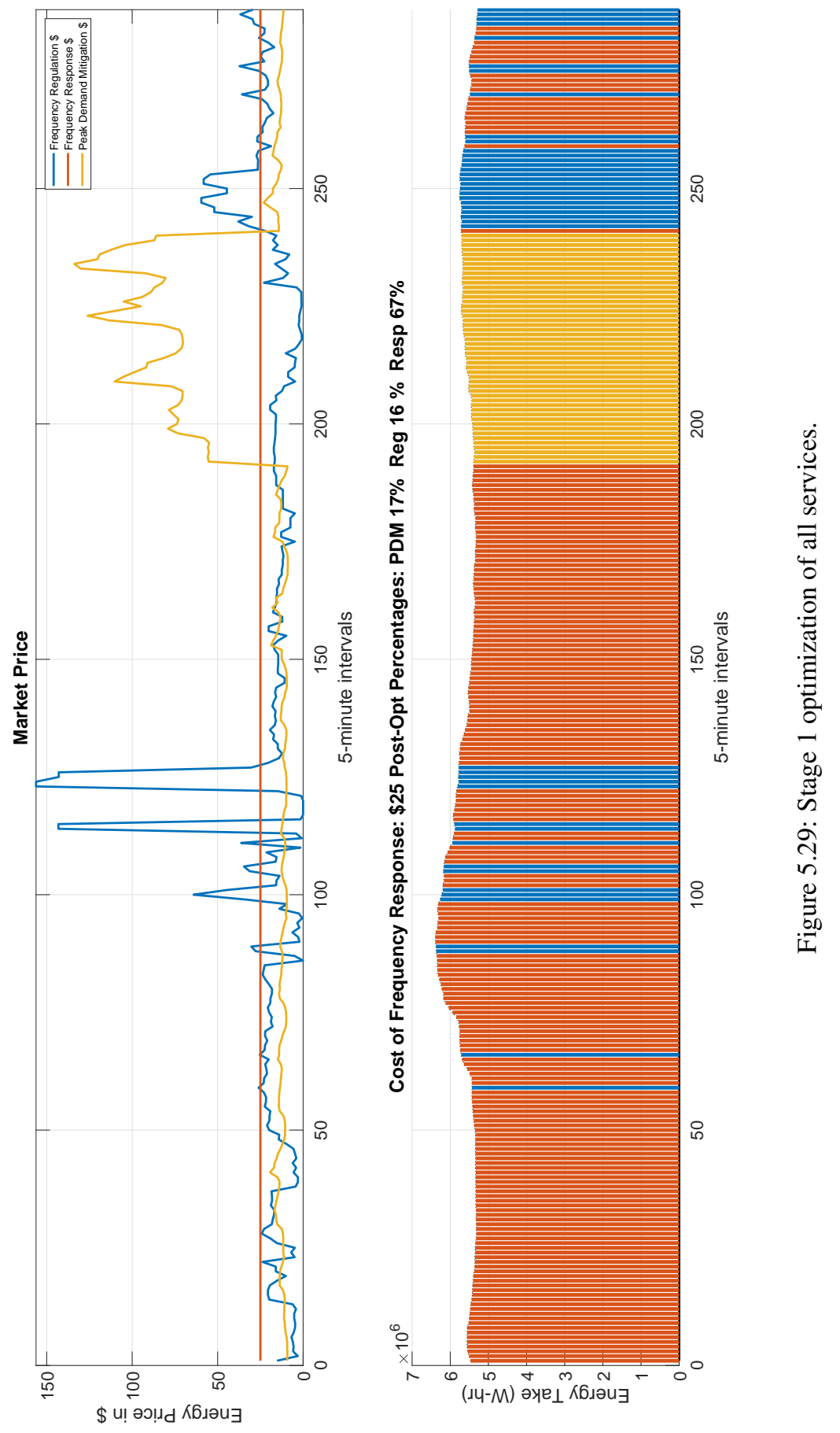



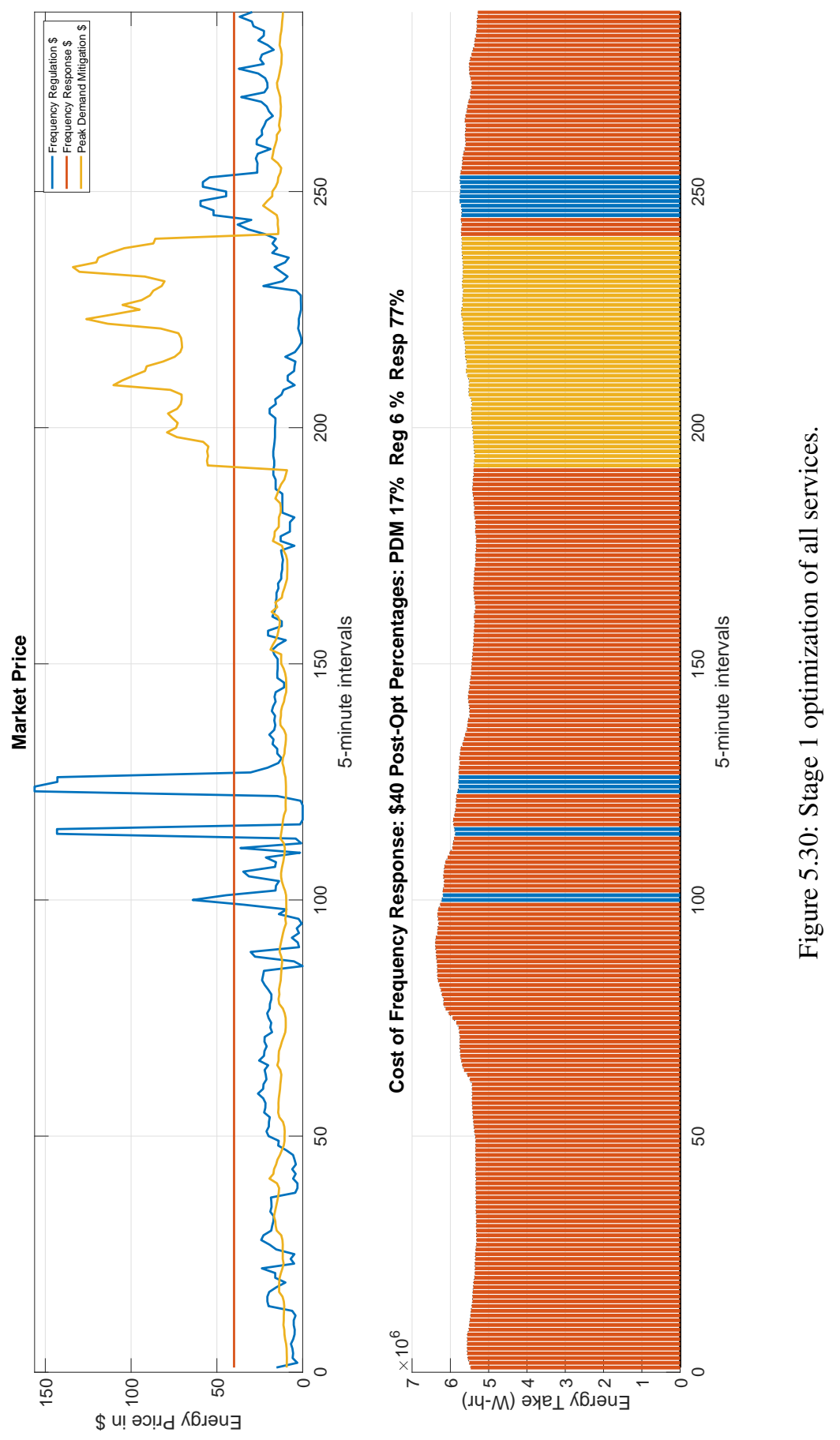


\subsubsection{Case 1.3 - Multi-service reservation}

In the multi-service case presented here, we attempt to reserve more than one service during the same time period. Multi-service reservation occurs due to the power and energy constraints that are defined for each service. In Figure 5.31, the optimization algorithm prioritizes dispatches based on the price of the services first, then it considers the boundaries placed on the respective services. Because we have specified that frequency regulation be limited to $1 \mathrm{MW}-\mathrm{hr}$ in this case, it will be dispatched for not more than $1 \mathrm{MW}-\mathrm{hr}$. We do this so as to make sure that we have enough reserves capacity for our reserve services, namely, peak demand mitigation and frequency response. 


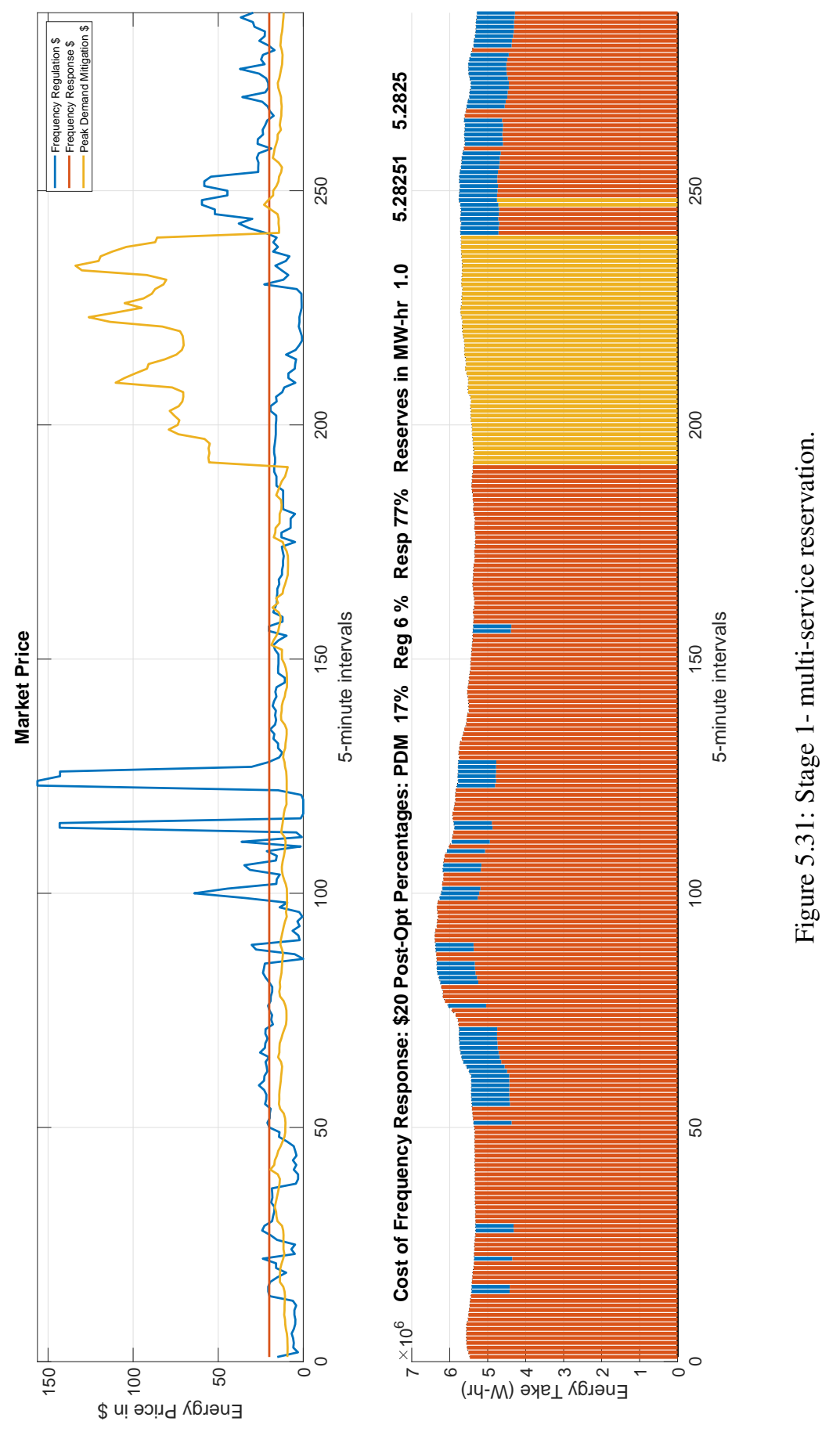


At the start of the optimization, at time interval 0 , frequency response is optimized but for only $1 \mathrm{MW}$-hr per five minute interval up until when regulation pays more than frequency response. Becaause of the bounds we have specified as reserve limits for frequency regulation, the optimization will not dispatch more than $1 \mathrm{MW}$ per five minute interval to frequency regulation, it then reserves the rest for frequency response.

We specify the maximum available energy take for each of the two reserve services but limit that of frequency regulation to $1 \mathrm{MW}$ per 5 minute interval or $12 \mathrm{MW}-\mathrm{hr}$.

We observe that at the start of peak demand period, all services are reserved for peak demand and no dispatches will happen during this period. Secondly, for the time periods immediately following peak demand mitigation (around time interval 249), the optimization algorithm limits the dispatches for peak demand mitigation to $1 \mathrm{MW}$ per five-minute interval, and at the same time interval 249 , it allocates the remainder of the energy take to peak demand mitigation. This is because peak demand was the next highest in price, slightly above frequency regulation as shown on the top market price plot.

\subsubsection{Case 1.4 - Bounding Frequency response price variations}

In this case, we vary the price of frequency response and observe how the optimization behavior changes based on the price of the different services and the reserve bounds set on frequency response. Frequency regulation is limited to dispatch no more than $1 \mathrm{MW}$-hr per five minute interval, while frequency response is reserved for no more than $2 \mathrm{MW}$-hr per five minute interval.

In Figure 5.32, frequency response price is at $\$ 20$. 


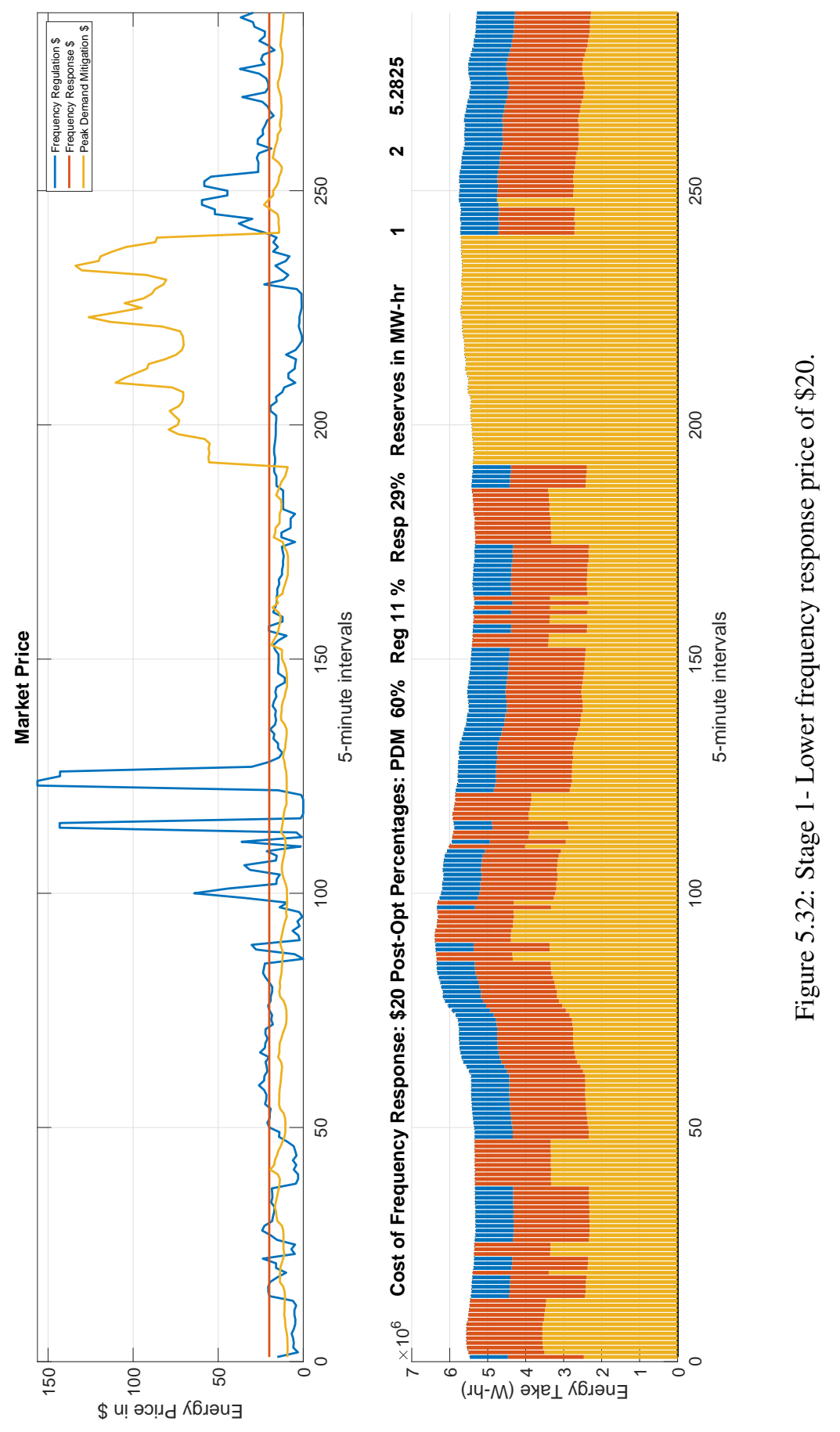


In Figure 5.33, the price of frequency response is doubled, going from $\$ 20$ to $\$ 40$. We remove the reserve requirement on frequency regulation and peak demand mitigation but we optimize all services based on market prices. Consistent with our expectations, because of the reserve requirement of $2 \mathrm{MW}$ per five minute interval placed on frequency response, despite its dominance in price, the optimization algorithm only allocated $41 \%$ of the daily energy take to frequency response. 

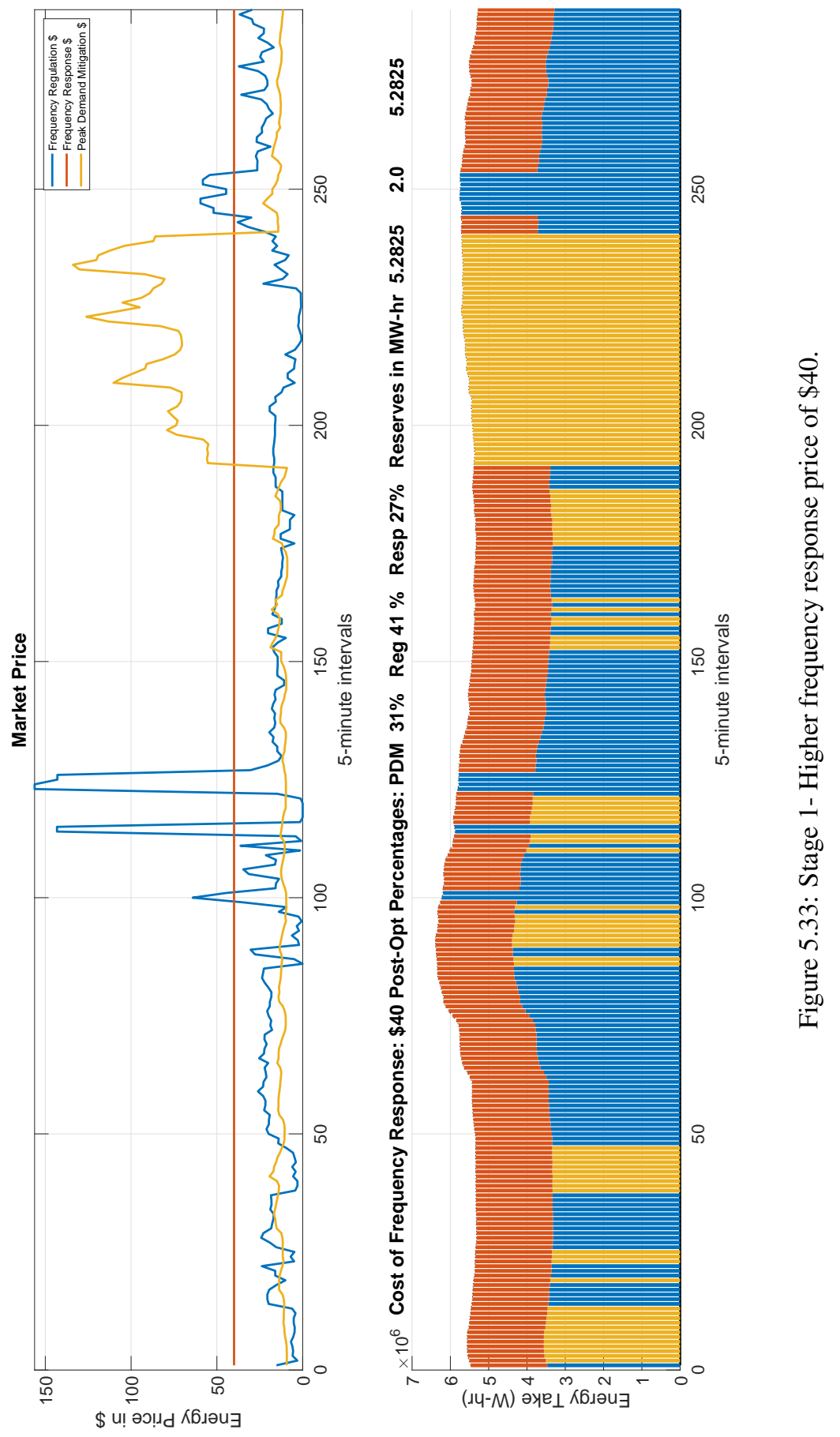


\subsubsection{Case 1.5 - Setting the upper boundary of frequency regulation}

In Figure 5.34, we set the upper boundary of frequency regulation to $0.17 \mathrm{MW}-\mathrm{hr}$. We see that the optimization algorithm schedules for dispatches according to the upper boundary limits specified for frequency regulation. 


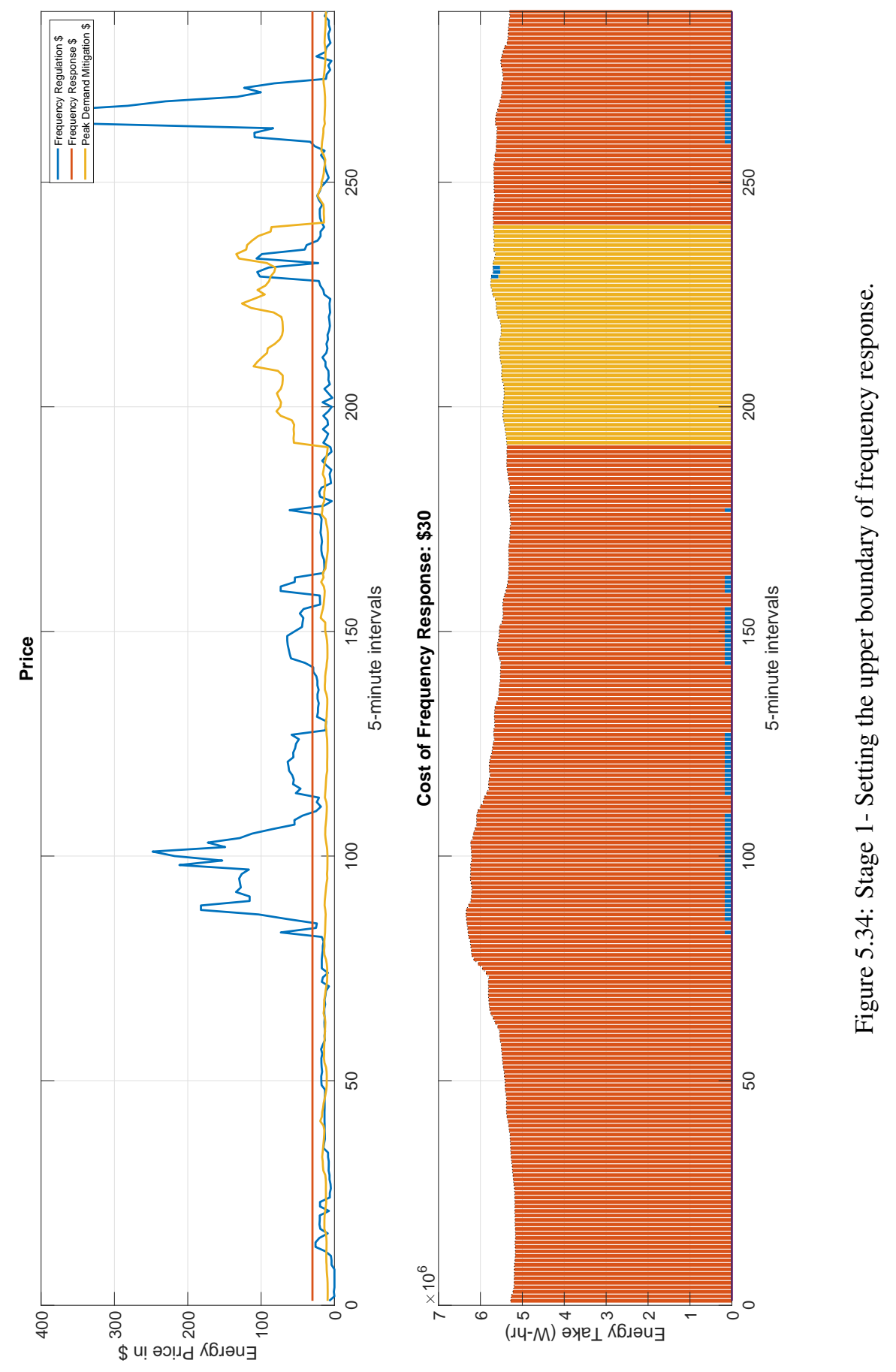




\subsubsection{Case 1.6 - Bounding Peak Demand Mitigation with variation in frequency response prices}

In Figure 5.35, Peak demand mitigation is manually reserved at $1 \mathrm{MW}$ per five minute interval. Because there is no dispatches in stage 1, Peak Demand Mitigation as a reserve service is set aside according to be boundary condition defined in the optimization algorithm. The price of peak frequency response is $\$ 20$. 


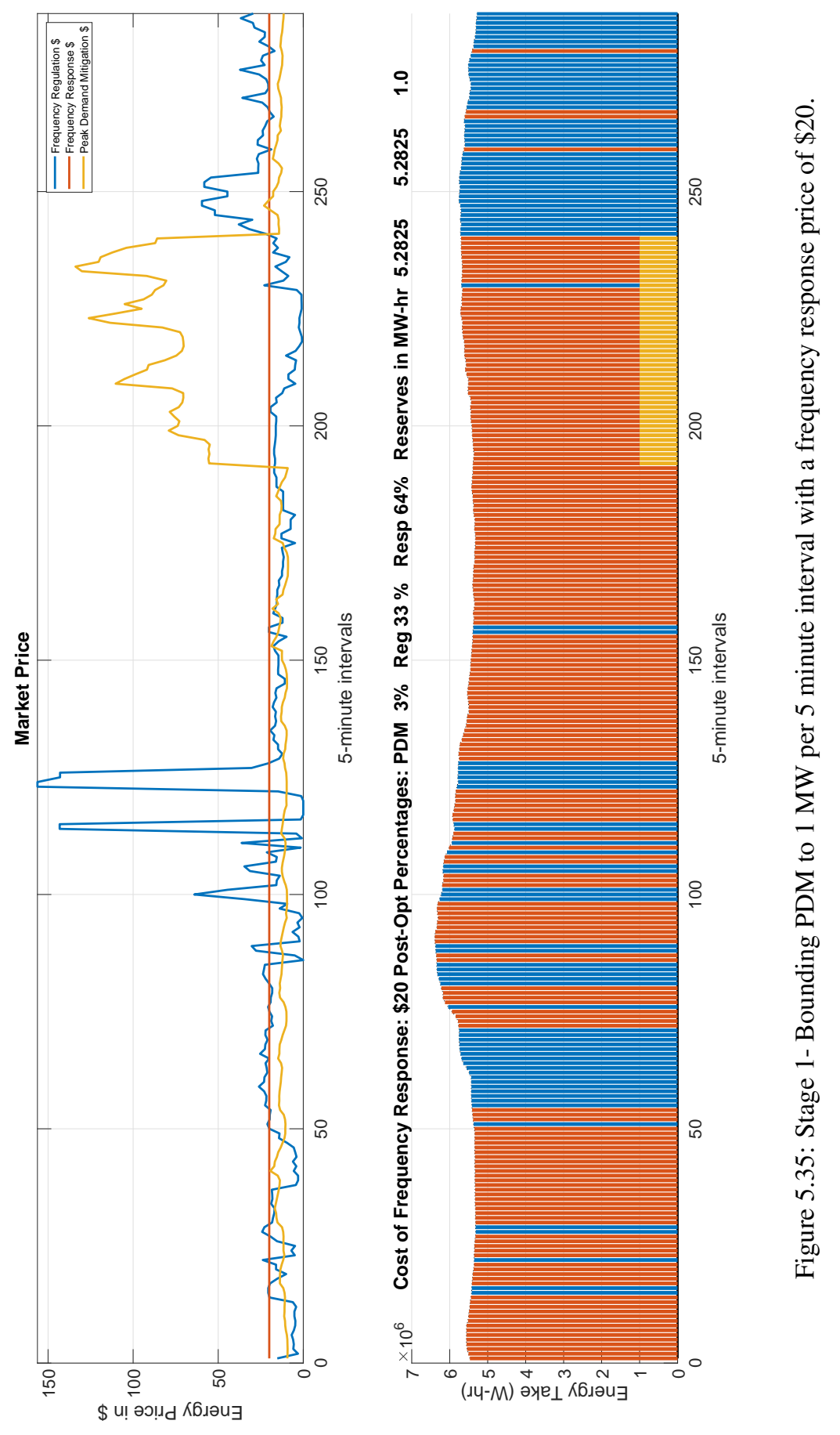


We double the price of frequency response from $\$ 20$ in Figure 5.35 to $\$ 40$ in Figure 5.36. We observe that because peak demand was mostly compensated only during the peak demand period only, as long as the price of peak demand is higher than that of frequency response during the peak demand window, there is no difference between Figure 5.35 and Figure 5.36. In both figures, only three percent of the energy take was allocated to peak demand mitigation. 


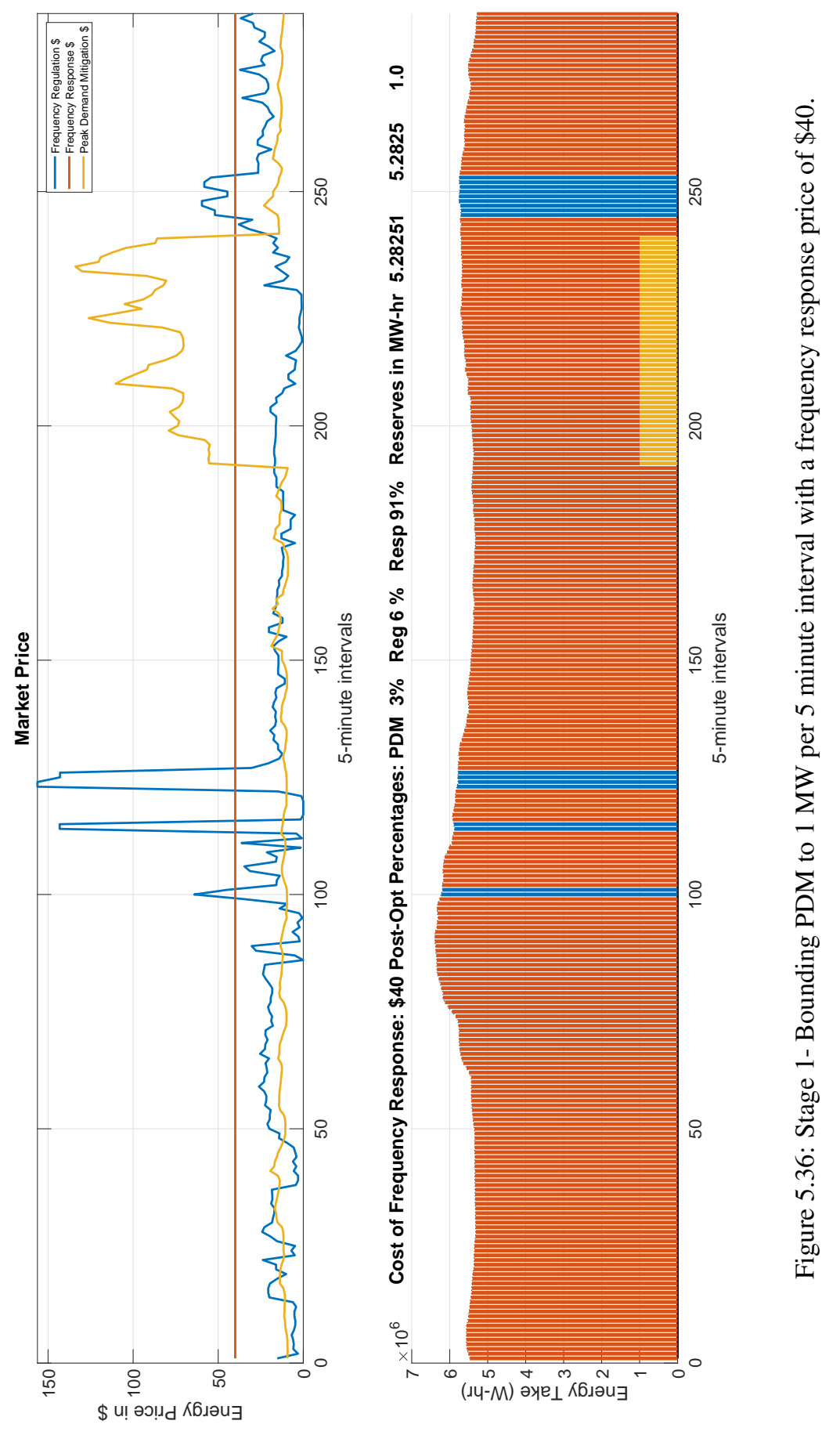




\subsection{Stage 2 Optimization}

In stage two, the AWHS is optimized to schedule the three services based on forecasted market prices, and the impact that the two dispatch services have on the AWHS energy take. As noted from earlier, two of the three services are reserves, and the other, frequency regulation, is a dispatchable services. Specifically, frequency response and peak demand mitigation are reserve services while frequency regulation is a dispatch services.

\subsubsection{Case 2.1 - 24 hour continuous dispatches with no upper limit boundary set on frequency regulation}

We test the limitations of the AWHS by continuously dispatching to the different services under consideration through out the day. Due to the fact that the AWHS is a dynamically changing resource, as it gets dispatched, the recovery curve is redrawn for each time interval and then the optimization is repeated.

We started the optimization in stage 2 without any bounds on frequency regulation. In Figure 5.37, the system is shown to quickly run out of energy take around time interval. 

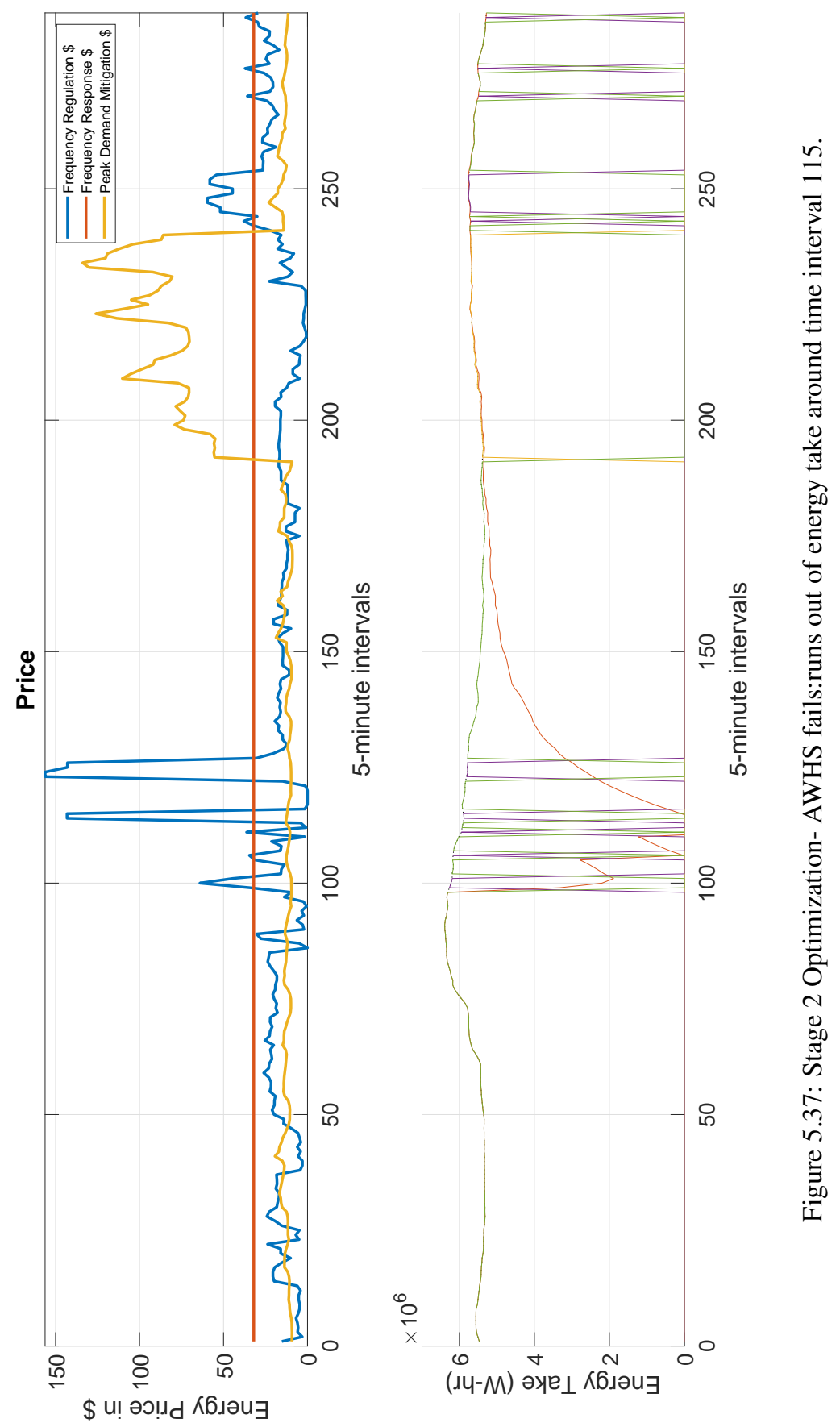


\subsubsection{Case 2.2 - 24 hour continuous dispatches with an upper limit boundary set on frequency regulation}

In this case, we set the upper boundary for frequency regulation dispatch. The upper boundary limits on frequency regulation is $2 \mathrm{MW}-\mathrm{hr}$ or $0.17 \mathrm{MW}$ per five minute interval, we re-ran the stage 2 optimization with the expectation that the AWHS does not run out of energy.

In Figures 5.38 we show that the AWHS dispatches for frequency regulation but then reserves energy as it recharges in accordance with frequency response and peak demand mitigation price signals. 

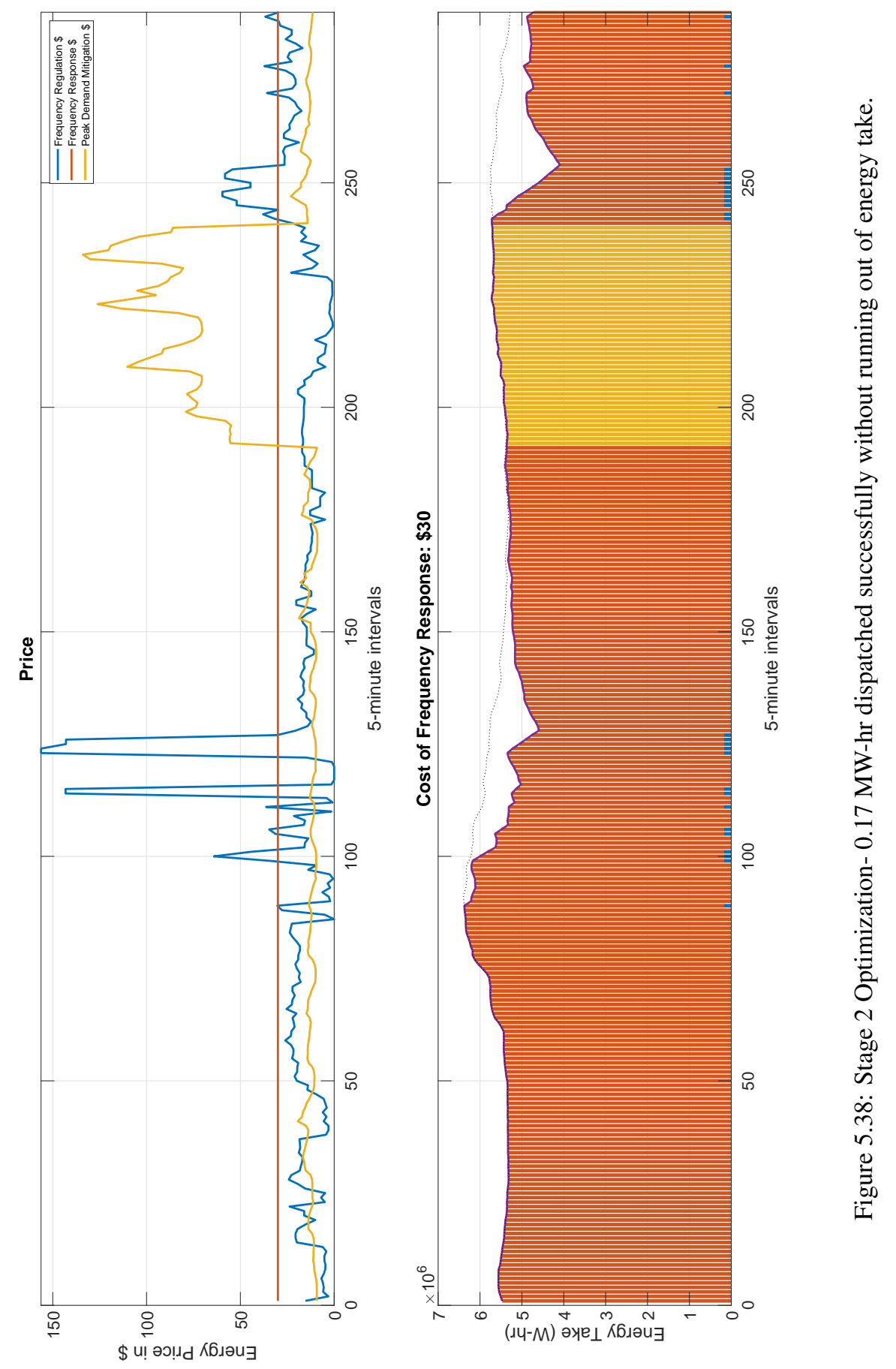
This is consistent with our observation from section 4.4.4 and Figure 4.3, that shows the maximum power available in the AWHS vs. the number of consecutive 5-minute dispatches. We have shown that there is a limit to the the continuous dispatch of the AWHS to 2.0 MW-hr, if the system is to not run out of energy take. Therefore, this case is intended to demonstrate a successful optimization and dispatch over an entire day in Figure 5.38. By setting the upper boundary of frequency regulation to $0.17 \mathrm{MW}-\mathrm{hr}$, the optimization algorithm successfully loops through out the day, dispatching both frequency regulation without running out of energy.

\subsubsection{Case 2.3 - Continuous dispatches over prolonged durations on a non-peak demand day}

In case 2.2, we noticed that both frequency response and peak demand mitigation were reserved. We reserved peak demand and avoided dispatching because during the peak demand period, there is a need to curtail all load and all load resources, including the AWHS. Dispatching means turning on the water heaters in the AWHS on, adding more loads to the grid can cause more stress on the already over-loaded system during this time.

In this case however, we explore the behavior of the AWHS on a non-peak demand day, in light of the fact that peak demand events are not a daily occurrence. In Figure 5.39, frequency regulation pricing is noticeably higher during the evening hours, around time interval 200. This is expected because at this time most people are returning home from work. This is the second peak described in the duck curve from Figure 1.1. Because of the 
upper boundary (0.17 MW-hr) we have placed on Frequency regulation, the AWHS never runs out of energy. 

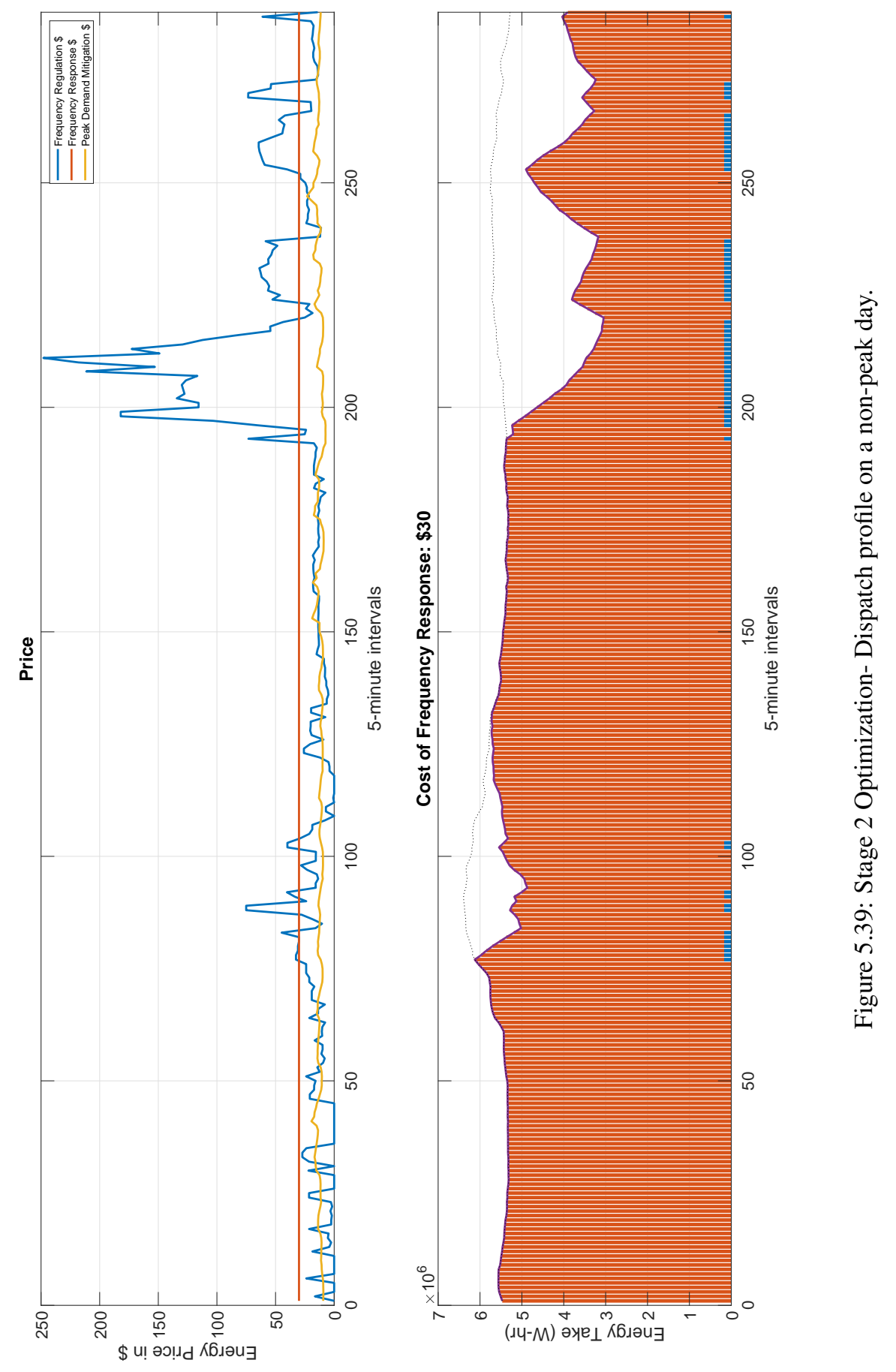


\subsubsection{Case 2.4 - Dispatch for Frequency Response}

Frequency response events are stochastic in nature and unpredictable. In Figures 5.40 and 5.41, we demonstrate that the optimizer is able to dispatch for frequency response events. As a reserved service, frequency response is reserved throughout the day by the optimization algorithm until a frequency response event occurs. In Figure 5.40, we show the AWHS resource profile with no frequency response event. 


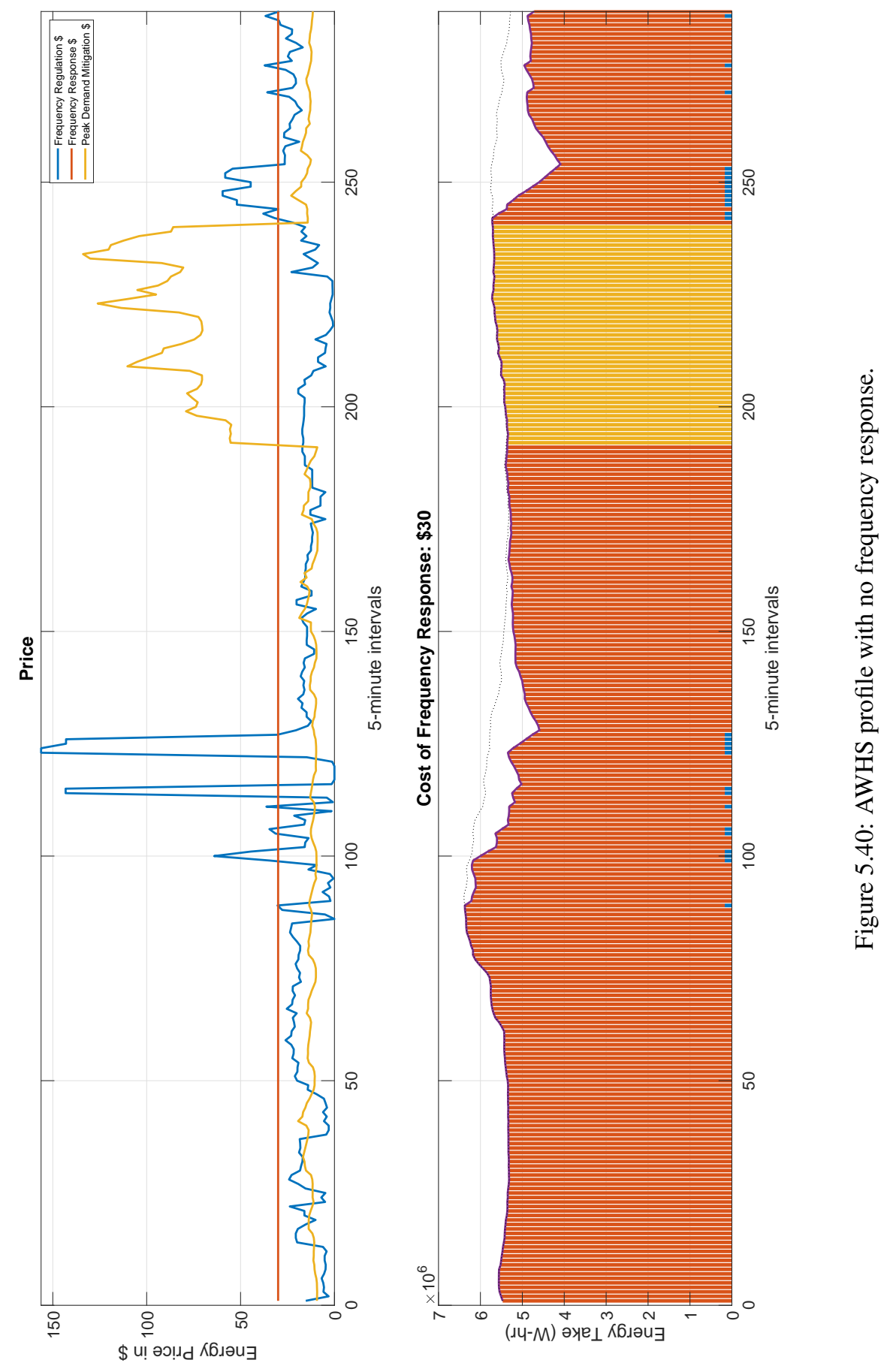


In Figure 5.41, we simulate a frequency response event around time interval 80. This frequency response event lasted for only one time interval, this is indicated by the fast drop in energy take value at time interval 80 . This unexpected frequency response event changed the energy take profile of the AWHS, thereby reducing the amount of revenue that could have been earned by the AWHS during the recovery period. 


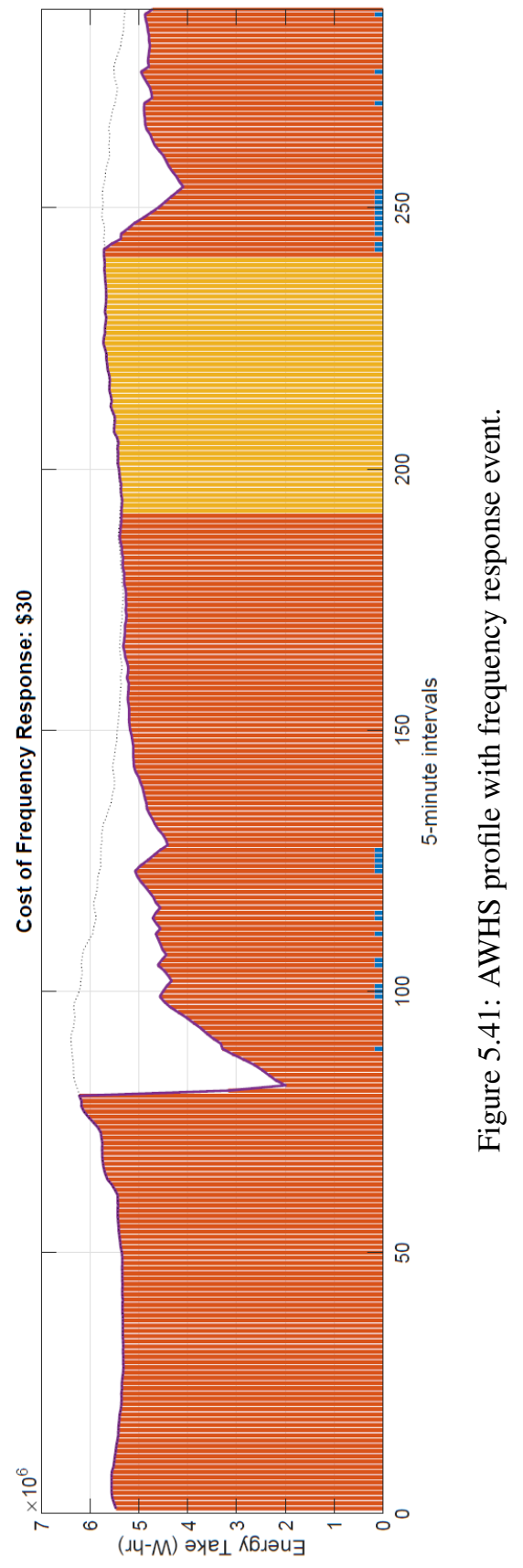




\subsection{Analysis of Revenues}

We conclude our research by comparing the revenues from the AWHS without the two-stage optimization to that obtained with the two-stage optimization applied in Table 5.4.

The derivation of revenues has been already established in equation 4.21;

$$
G_{i}=\left(a_{i} P_{1 i}+b_{i} P_{2 i}+c_{i} P_{3 i}\right) \triangle t_{i}
$$

\begin{tabular}{|l|l|l|l|l|}
\hline $\begin{array}{l}\text { Frequency response, } \\
\text { market price in } \\
\text { \$ MW-hr }\end{array}$ & $\begin{array}{l}\text { Frequency regulation, } \\
\text { no optimization, } \\
\text { revenues in \$ }\end{array}$ & $\begin{array}{l}\text { Frequency response, } \\
\text { no optimization, } \\
\text { revenues in \$ }\end{array}$ & $\begin{array}{l}\text { Peak demand, } \\
\text { no optimization, } \\
\text { revenues in \$ }\end{array}$ & $\begin{array}{l}\text { two-stage } \\
\text { optimization, } \\
\text { revenues in \$ }\end{array}$ \\
\hline 10 & 3,162 & 1617 & 4,831 & 6,929 \\
\hline 20 & 3,162 & 3227 & 4,831 & 7,083 \\
\hline 30 & 3,162 & 5172 & 4,831 & 7,846 \\
\hline 40 & 3,162 & 4849 & 4,831 & 12,946 \\
\hline 50 & 3,162 & 6468 & 4,831 & 13,281 \\
\hline 100 & 3,162 & 16165 & 4,831 & 16,165 \\
\hline
\end{tabular}

Table 5.4: Comparison of the revenues earned by the AWHS without the two-stage optimization vs. the AWHS with the two-stage optimization using the market prices from July 14, 2016.

Each row in Table 5.4 shows the results from an entire day's worth of simulation. The first column represents the market prices of frequency response which we have elected to represent as a constant throughout the day, for each simulation. The market prices used for frequency regulation and peak demand mitigation were obtained from PJM's July 14, 2016 LMP data, and they are not shown in Table 5.4 because they fluctuate throughout the entire day. Revenues earned by the AWHS are represented in the second, third, fourth and fifth column.

As the market price of frequency response increased for each simulation, as we go down Table 5.4, the revenues generated for both frequency regulation and peak demand mitigation remained flat. The explanation for this is that these revenues both represent the performance 
of the AWHS without the two-stage optimization applied. However, revenues from frequency response reservations without the two-stage optimization increased as the market prices for frequency response increased. In other words, with no two-stage optimization applied, as we artificially increased the market price of frequency response, the revenues generated by reserving the AWHS for frequency response alone increased. This outcome is consistent with our expectations.

The revenues earned by the AWHS with the two-stage optimization is represented in the rightmost column of Table 5.4. For the two-stage optimization, the AWHS is allowed to automatically select the most optimal of the three ancillary services based on market price signals. As shown in Table 5.4, the AWHS generates the most revenue via the two-stage optimization, up until when the price of frequency is the highest among all three services. When this occurs, as expected, the two-stage optimization will select the service with the highest price. This is what happens when the market price for frequency regulation is at $\$ 100$ in the last row of Table 5.4, at which point, frequency response revenues was at par with the revenues obtained from the two-stage optimization revenues because the two-stage optimization algorithm only selected frequency response throughout the entire day's worth of simulation.

Most utilities bid into the energy market using forecasts or based on actual real time loading demands, without our novel two-stage optimization algorithm. In contrast, our two-stage optimization uses time shifting and re-optimizes dynamically, for each five minute time interval, allowing the AWHS resource to account for market price signal fluctuations, 
while only selecting the service that will earn the most revenue, at all times. The former however, may not always yield the most revenue.

In summary, the limitation of the two-stage optimization is reached when any particular service has the highest market price, at all times, throughout the day. In the scenario when the frequency response market price becomes $\$ 100$ per MW-hr in Table 5.4, dedicated resources providing services to frequency alone will benefit as much as our two-stage optimization algorithm, as long as the market price of frequency response remains the highest in comparison to the other two services throughout the day, as was the the case on July 14, 2016. In practise however, market prices always fluctuate throughout the entire day because weather (wind speed and solar irradiation), system loading and market conditions are never constant.

\subsection{What would the value be of including other ancillary services?}

The value of including a service such as ramp rate and reserves would be the potential for increased revenues for the AWHS through greater market participation. Another benefit would have been an increase in penetration of the AWHS to more service areas. Not including these services limits the energy markets that the AWHS can participate in, along with the learning that comes with such engagements.

Through this work, we have been able to observe the behavior of the AWHS for frequency regulation, frequency response and peak demand mitigation in terms of how revenue can be maximized between these services. With ramp rate and reserves however, we anticipate that 
there will be more value streams to the AWHS in addition to revenue maximization, like grid stability and improvements to system wide reliability, both of which can be improved were ramp rate and reserves to be included as part of the services offered by the AWHS.

\subsection{Choice of Linear Programming constraints}

The constraints modeled in our LP solution were added to prevent over-dispatching, and over-dispatching can lead to increased opportunity costs for the AWHS. Both of these will reduce the AWHS revenue. There are a few other constraints that could have been included however.

For example, limiting peak demand mitigation to a certain four-hour period, although not very efficient, was added as a constraint because this is the current industry practice. Other constraints that could have been added were locational benefits or distribution system capacity limits. In the former, depending on the location of the customer and distance from generators, the optimization algorithm can be modelled to behave differently. For the later, the AWHS can be modelled to split its resources, dedicating a higher portion to regions near or at their distribution system capacity limits. Both of these were omitted because they would require more complex modelling and a sophisticated algorithm.

\subsection{What would other objectives have been?}

The objective of this work is to alleviate the negative impacts of RER on the grid by optimally dispatching the AWHS to provide ancillary services in a manner that maximizes revenues. 
Other objectives could have been to do this work in a way that maximises profit and not revenue. We chose revenue over profit because in order to calculate profit, we need to know costs. Costs not only vary between customers, but costs can vary significantly across different water heater OEMs, water heater efficiencies, age of units, States, and Regions. We chose not to include costs as an objective because including costs will not make the optimization very complex, but it might require constant modification depending on the manufacturer, water heater efficiency, age, State or Regional application, thereby limiting the broad application of our two-stage optimization.

\subsection{Shortcomings of this work}

The AWHS simulates the behavior of 10,000 water heaters, and as such, may not accurately replicate the exact representation of 10,000 physically networked water heaters. This can be addressed by using real water heaters.

Another shortcoming of this work is that ambient losses were neglected. With 10,000 water heaters in the AWHS, external losses will vary amongst all units based on the disparate locations of all the water heaters, geographically and within each home. This shortcoming can be overcome by including a normalized loss function that can be applied to the AWHS model. 


\section{Conclusion}

In this work, we propose a means of solving the problems caused due to the excessive proliferation of Renewable Energy Resources (RER) by using an Aggregated Water Heater System (AWHS), a regenerating and dynamic resource, while optimizing the AWHS for revenue based on market price signals by providing three ancillary services.

Our motivation is based on the growing need to relieve the electric grid of the negative impacts of RER. We chose to embark on this work as a means of using residential water heaters to provide ancillary services to the electric grid. We have shown that the stochastic nature of RER have several negative impacts on system reliability and stability of the electric grid. The means of mitigating these impacts must be developed if we are to continue the rapid deployment of RER and global decarbonization efforts.

We began our research by exploring existing solutions to this problem, some of which include Energy Storage Systems (ESS), the current practice of using dedicated Traditional Generation Resources (TGR) for ancillary services support, Dispatchable Standby Generation (DSG), Hybrid Energy Systems (HES), Demand Side Management (DSM), and Asset Aggregation (AA). We focused our attention on AA, specifically, the aggregation of thousands of residential resistance Electric Water Heaters (EWH). Resistance EWH were chosen because they are ubiquitous, and they have been shown to be quick to respond to grid control signals. 
With AA using EWH identified as our path to tackling the challenges caused by RER, we formulated our primary research question as follows: How should a water heater load resource be allocated between three ancillary services markets, frequency regulation, frequency response and peak demand mitigation, at any given time of day based on the available energy take among 10,000 water heaters in such a way that dispatch is prioritized to maximize revenue? Revenue is maximized by determining the optimal ratio of ancillary services to provide during each five-minute interval based on market price signals.

The primary contribution to knowledge is a solution to the problem caused by RER that uses a novel two-stage method to optimally dispatch the energy take available in the AWHS among three ancillary services in a way that maximizes revenue while minimizing over dispatching, system recovery time and energy take forecasting errors.

Our results show that water heaters can be used to participate in decremental dispatches into the frequency regulation market in an optimal fashion. We have also shown that placing reserves and limiting the amount of energy to dispatch can be useful on days with higher than usual dispatches of the AWHS. The optimization algorithm has been shown to maximise revenue by selecting the optimal of the three services to dispatch or reserve based on market price signals. 


\section{Future Work}

A major challenge encountered in this work was that of computer processing speed limitations. Due to the large computation involved, we found that our optimization algorithm took an average of about 4 to 5 hours to complete. This was because our method required that we constantly update the operational vectors for each time shift, and that we generate new vectors using forecasted prices for each iteration. In addition, for every dispatch, the AWHS was updated with new energy take values, and the resource recovery curve was redrawn, requiring a large amount of computer memory allocation. Given that this work is still at an early stage, this challenge should be addressed in order for further development to continue, however, with adequate resources and a dedicated data center, as is the case with most utilities and Independent System Operators (ISO), we suspect that this may not be an issue.

Second, because we used MATLAB ${ }^{\circledR}$ for all our simulations, we found that the stacked bar plot function used for displaying our results was not able to correctly allocate the AWHS resources when multiple services were reserved during the same time interval. Further, for the Gigabyte order of data that we worked with, the latency in polling and retrieving large datasets was not optimal. We suggest using Python or $R$ for future work, as these are both more suitable for handling large computations, they both have more optimization functions and can support forecasting and large data analysis more efficiently. 
Another area that could be explored further is the addition of other services like ramp rate, reserves and energy as a service to the optimization routine. We discussed the benefits of including ramp rate and reserves in section 5.10. For adding energy as a fourth service, Locational Marginal Pricing (LMP) data can be fed into the optimization algorithm developed in this work. This can be very profitable for load resources like the AWHS during periods of negative energy prices. Negative energy prices are caused by a combination of wind or hydro over-generation and low demand on the wholesale exchange energy market, and is common in regions with high penetration of wind or hydro like Texas, the Pacific Northwest and in Countries like Germany.

The minimum dispatch amount that will prevent over-dispatching of the AWHS, and the convergence percentage of choice can be explored in future work, in this research however, we chose $2.0 \mathrm{MW}-\mathrm{hr}$ and $5 \%$ respectively. In addition, the energy take decay solution discussed in section 4.6.4 can be further explored in the future.

We currently use Locational Marginal Pricing (LMP) to provide a market signal for Peak Demand Mitigation (PDM), which is a reserve service. High LMP during designated PDM time periods signal to the AWHS that water heaters should not be turned on. In addition to PDM though, we could also use LMP for several other services, which we propose in the following paragraphs.

At times, particularly during Spring in the Pacific Northwest, LMP prices can become negative, for instance due to high wind and hydro generation. This is a signal to generation that there is surplus power being provided to the system, so the negative price disincentives 
generation. Concurrently, negative prices incentivize loads to consume energy; essentially, loads are paid to turn on. System equilibrium is achieved with generation and loads respond to these negative prices. As such, we can position the AWHS to use this phenomena as a revenue opportunity by programming the AWHS to turn on water heaters as a dispatch service when LMP become negative.

In this case though, the revenue from dispatch must be weighed against the opportunity costs in the near term due to reduced AHWS energy take. If dispatch removes energy take that could be used in subsequent hours to earn revenue through scheduling a reserve service, then the dispatch should not be scheduled if the revenue from dispatch is less than the revenue that could be earned through scheduling a reserve service.

Another opportunity for using LMP to signal scheduling of the AWHS could come from a service we call Solar Valley Filling (SVF). Consider Figure 1.3, which shows solar resources lowering the demand curve during midday and a subsequent peak in the evening, featuring a high ramp rate in the interim. To provide SVF, we could use LMP to signal the AWHS to dispatch water heaters during midday if we anticipate LMP to be high in the evening. Effectively, water heaters would preheat in anticipation of the upcoming evening ramp. A forward-looking vector of forecast evening prices could be compared to an immediate vector of forecast midday prices to establish a difference price vector that would serve as the price signal. As such, SVF would provide another revenue stream for the AWHS.

Contrary to the negative pricing case discussed above wherein opportunity costs must 
be considered against the value of LMP, SVF would not have to be considered against scheduling alternative services. SVF would dispatch during midday and reserve an equal amount of energy take during the evening ramp period. In other words, a bid for SVF would schedule two time periods: a dispatch period during midday to mitigate excess solar generation and a reserve period during the evening to mitigate excess loading.

Lastly, we recommend that a multi-objective optimization be developed for the AWHS. This can be viable in scenarios that along with optimizing revenue, one or all of the ancillary services can also be concurrently optimized, based on whether the service is to be dispatched or reserved at the offered market price. This will help better understand the opportunity costs involved when one service is either being dispatched or reserved with the knowledge of the dynamic market prices for such services and the overall impact to the AWHS energy take recovery times. 


\section{Bibliography}

[1] T. Clarke. Aggregation of Electric Water Heaters for Peak Shifting and Frequency Response Services. Portland State University, 2019. Masters thesis.

[2] CAISO. Demand response and energy efficiency roadmap: Maximizing preferred resources. California Independent System Operator, December 2013.

[3] Bonneville Power Administration. Description of Electric Energy Use in SingleFamily Residences in the Pacific Northwest. Technical report, 1992.

[4] K. Marnell. Large-Scale DER Aggregations of Electric Water Heaters and Battery Inverter Systems. Portland State University, 2019. Masters thesis.

[5] CTA-2045 water heater demonstration report including a business case for CTA-2045 market transformation. Bonneville Power Administration, project 336, November 2018.

[6] E. Ela and D. Edelson. Participation of Wind Power in LMP-Based Energy Markets. IEEE Transactions on Sustainable Energy, 3(4):777-783, 2012.

[7] E. Bitar, R. Rajagopal, and P. Varaiya. Bringing Wind Energy to Market. IEEE Transactions on Power Systems, 27(3):1225-1235, 2012. 
[8] D. Rastler. Electricity energy storage technology options: A white paper primer on applications, costs, and benefits. Technical report, Electric Power Research Institute, Palo Alto, CA, 2010.

[9] G. Liu and K. Tomsovic. Quantifying Spinning Reserve in Systems With Significant Wind Power Penetration. IEEE Transactions on Power System, 27(4):2385-2389, 2012.

[10] B.C. Ummels, M. Gibescu, E. Pelgrum, W.L. Kling, and A.J. Brand. Impacts of Wind Power on Thermal Generation Unit Commitment and Dispatch. Energy Conversion, IEEE Transactions on, 22(1):44-51, 2007.

[11] H. Xin, Z. Lu, Y. Liu, and D. Gan. A center-free control strategy for the coordination of multiple photovoltaic generators. IEEE Transactions on Smart Grid, 5(3):12621269, May 2014.

[12] P.P. Zarina, S. Mishra, and P.C.Sekhar. Deriving inertial response from a non-inertial pv system for frequency regulation. Power Electronics, Drives and Energy Systems (PEDES), IEEE International Conference on, pages 1245-1249, December 2012.

[13] M K Hossain and M H Ali. Small scale energy storage for power fluctuation minimization with spatially diverged PV plants. Southeastcon, 2013 Proceedings of IEEE, pages 1-6, April 2013. 
[14] S. Nandkeolyar, R. K. Mohanty, and V. A. Dash. Management of time-flexible demand to provide power system frequency response. In 2018 Technologies for Smart-City Energy Security and Power (ICSESP), pages 1-4, March 2018.

[15] A.Akhil, G. Huff, A. Currier, B. Kaun, D. Rastler, S. Chen,. DOE/EPRI electricity storage handbook in collaboration with NRECA. Sandia National Laboratories. 2015.

[16] K. Marnell, M. Obi, and R. Bass. Transmission-scale battery energy storage systems: A systematic literature review. Energies, 12(23):4603, December 2019.

[17] L. Liang, J. Zhong, and Z. Jiao. Frequency regulation for a power system with wind power and battery energy storage. In 2012 IEEE International Conference on Power System Technology (POWERCON), pages 1-6, Oct 2012.

[18] L. Wu, W. Gao, Z. Cui, and X. Kou. A novel frequency regulation strategy with the application of energy storage system for large scale wind power integration. In 2015 Seventh Annual IEEE Green Technologies Conference, pages 221-226, April 2015.

[19] F. Díaz-González, M. Hau, A. Sumper, and O. Gomis-Bellmunt. Participation of wind power plants in system frequency control: Review of grid code requirements and control methods. Renewable and Sustainable Energy Reviews, 34:551 - 564, 2014.

[20] L. Holt and M. Galligan. States' RPS policies: Serving the public interest? The Electricity Journal, 26:16-23, December 2013. 
[21] G. Barbose. Renewables portfolio standards in the united states: A status update. In State-Federal RPS Collaborative National Summit on RPS. Lawrence Berkeley National Laboratory, 6 November 2013.

[22] H. Wang and X. Bai. Adequacy assessment of generating systems incorporating wind, PV and energy storage. In 2012 IEEE Innovative Smart Grid Technologies - Asia (ISGT Asia), pages 1-6, 21 -24 May 2012.

[23] F. Diaz-Gonzalez, A. Sumper, O. Gomis-Bellmunt, and R. Villafafila-Robles. A review of energy storage technologies for wind power applications. Renewable and Sustainable Energy Reviews, 16(4):2154-2171, 2012.

[24] DOE/EPRI. Electricity storage handbook in collaboration with the national rural electric cooperative association, available: www.sandia.gov/ess/publications/sand20135131.pdf. [online], July 2013. [Accessed: April 5, 2018].

[25] C. Yang and R. B. Jackson. Opportunities and barriers to pumped-hydro energy storage in the United States. Renewable and Sustainable Energy Reviews, 15(1):839 844, 2011.

[26] T. Trainer. Some problems in storing renewable energy. Energy Policy, 110:386 $393,2017$.

[27] T. Ma, H. Yang, and L. Lu. Feasibility study and economic analysis of pumped hydro storage and battery storage for a renewable energy powered island. Energy Conversion and Management, 79:387 - 397, 2014. 
[28] Z. Ming, Z. Kun, and W. Liang. Study on unit commitment problem considering wind power and pumped hydro energy storage. International Journal of Electrical Power \& Energy Systems, 63:91 - 96, 2014.

[29] Y. Zhou, C. Xia, H. Zhao, S. Mei, and S. Zhou. An iterative method for evaluating air leakage from unlined compressed air energy storage caverns. Renewable Energy, 120:434 -445, 2018.

[30] E. Fertig and J. Apt. Economics of compressed air energy storage to integrate wind power: A case study in ERCOT. Energy Policy, 39(5):2330 - 2342, 2011.

[31] J. M. Arroyo and A. J. Conejo. Modeling of start-up and shut-down power trajectories of thermal units. IEEE Transactions on Power Systems, 19(3):1562-1568, Aug 2004.

[32] I. Guisández, J.I. Pérez-Díaz, and J. R. Wilhelmi. Assessment of the economic impact of environmental constraints on annual hydropower plant operation. Energy Policy, 61:1332-1343, 2013.

[33] B. E. Ghazzani, D. M. Plaza, R. A. El-Cadi, A. Ihlal, B. Abnay, and K. Bouabid. Thermal plant based on parabolic trough collectors for industrial process heat generation in morocco. Renewable Energy, 113:1261 - 1275, 2017.

[34] F. Alsokhiry and K. L. Lo. Distributed generation based on renewable energy providing frequency response ancillary services. In 4th International Conference on Power Engineering, Energy and Electrical Drives, pages 1200-1205, May 2013. 
[35] Federal Energy Regulatory Commission. Essential Reliability Services and the Evolving Bulk-Power System Primary Frequency Response. 160 FERC פ661,011. Docket No. RM16-6-000, 2017.

[36] U.S. Net Generation from Renewable Sources: Total (All Sectors), 2004-October 2014. U.S. Energy Information Administration, December 2014.

[37] Energy Storage Association. ESA Comments on FERC's Essential Reliability Services and the Evolving Bulk-Power System Primary Frequency Response. 160 FERC I61,011. Docket No. RM16-6-000, 2017.

[38] M. Obi and R. Bass. Trends and challenges of grid-connected photovoltaic systems a review. Renewable and Sustainable Energy Reviews, 58, 052016.

[39] NERC. Nerc operation manual. North American Electrical Reliability Corporation, August 2016.

[40] M. Osborn. Experiences in aggregating distributed generation for system benefit. In IEEE Power Engineering Society General Meeting, 2004., pages 886-889 Vol.1, June 2004.

[41] K. Al-Salim, I. Andonovic, and C. Michie. Cyclic blackout mitigation and prevention using semi-dispatchable standby generation and stratified demand dispatch. Sustainable Energy, Grids and Networks, 4:29 - 42, 2015.

[42] M. Vaiman, P. Hines, J. Jiang, S. Norris, M. Papic, A. Pitto, Y. Wang, and G. Zweigle. Mitigation and prevention of cascading outages: Methodologies and practical 
applications. In 2013 IEEE Power Energy Society General Meeting, pages 1-5, July 2013.

[43] M. Koenig, P. Duggan, J. Wong, M. Y. Vaiman, M. M. Vaiman, and M. Povolotskiy. Prevention of cascading outages in Con-Edison's network. In IEEE PES T\& D 2010, pages 1-7, April 2010.

[44] M. Vaiman, K. Bell, Y. Chen, B. Chowdhury, I. Dobson, P. Hines, M. Papic, S. Miller, and P. Zhang. Risk assessment of cascading outages: Methodologies and challenges. IEEE Transactions on Power Systems, 27(2):631-641, May 2012.

[45] K. Ardani, E. Shaughnessy, and P. Schwabe. Coordinating distributed energy resources for grid services - a case study of pacific gas and electric. National Renewable Energy Lab Technical report NREL/TP-7A40-72108, November 2018.

[46] N. Mahmoudi, E. Heydarian-Forushani, M. Shafie-khah, T. K. Saha, M.E.H. Golshan, and P. Siano. A bottom-up approach for demand response aggregators' participation in electricity markets. Electric Power Systems Research, 143:121 - 129, 2017.

[47] A. M. Carreiro, H. M. Jorge, and C. H. Antunes. Energy management systems aggregators: A literature survey. Renewable and Sustainable Energy Reviews, 73:1160 $-1172,2017$.

[48] S. Burger, J. P. Chaves-Ávila, C. Batlle, and I.J. Pérez-Arriaga. A review of the value of aggregators in electricity systems. Renewable and Sustainable Energy Reviews, 77:395 - 405, 2017. 
[49] Y. Shimomura, Y. Nemoto, F. Akasaka, R. Chiba, and K. Kimita. A method for designing customer-oriented demand response aggregation service. CIRP Annals, 63(1):413-416, 2014.

[50] A. C. Chapman, G. Verbič, and D. J. Hill. Algorithmic and strategic aspects to integrating demand-side aggregation and energy management methods. IEEE Transactions on Smart Grid, 7(6):2748-2760, Nov 2016.

[51] Hledik R. \& Lueken R. \& Chang, J. The hidden battery: Opportunities in electric water heating. The Brattle Group, January 2016.

[52] Shi Y. Kirschen D. Zhang B. Xu, B. Optimal battery participation in frequency regulation markets. IEEE Transactions on Power Systems(33, no. 6, November 2018.

[53] G.C Bakos and N.F Tsagas. Technoeconomic assessment of a hybrid solar/wind installation for electrical energy saving. Energy and Buildings, 35(2):139 - 145, 2003.

[54] M.K. Deshmukh and S.S. Deshmukh. Modeling of hybrid renewable energy systems. Renewable and Sustainable Energy Reviews, 12(1):235 - 249, 2008.

[55] M.A. Abdullah, K.M. Muttaqi, and A.P. Agalgaonkar. Sustainable energy system design with distributed renewable resources considering economic, environmental and uncertainty aspects. Renewable Energy, 78:165 - 172, 2015.

[56] H.N. Post and M.G. Thomas. Photovoltaic systems for current and future applications. Solar Energy, 41(5):465 - 473, 1988. 
[57] M. Malinowski, J. I. Leon, and H. Abu-Rub. Solar photovoltaic and thermal energy systems: Current technology and future trends. Proceedings of the IEEE, 105(11):2132-2146, Nov 2017.

[58] H. Yang, L. Lu, and W. Zhou. A novel optimization sizing model for hybrid solar-wind power generation system. Solar Energy, 81(1):76 - 84, 2007.

[59] T. Senjyu, T. Nakaji, K. Uezato, and T. Funabashi. A hybrid power system using alternative energy facilities in isolated island. IEEE Transactions on Energy Conversion, 20(2):406-414, June 2005.

[60] R. Karki and R. Billinton. Reliability/cost implications of PV and wind energy utilization in small isolated power systems. IEEE Power Engineering Review, 21(12):67-67, Dec 2001.

[61] P. Nema, R.K. Nema, and S. Rangnekar. A current and future state of art development of hybrid energy system using wind and PV-solar: A review. Renewable and Sustainable Energy Reviews, 13(8):2096 - 2103, 2009.

[62] W. Zhou, C. Lou, Z. Li, L. Lu, and H. Yang. Current status of research on optimum sizing of stand-alone hybrid solar-wind power generation systems. Applied Energy, 87(2):380 - 389, 2010.

[63] M. W. Gustafson, J. S. Baylor, and G. Epstein. Direct water heater load controlestimating program effectiveness using an engineering model. IEEE Transactions on Power Systems, 8(1):137-143, Feb 1993. 
[64] B. F. Hastings. Ten years of operating experience with a remote controlled water heater load management system at Detroit Edison. IEEE Transactions on Power Apparatus and Systems, PAS-99(4):1437-1441, July 1980.

[65] J. R. Stitt. Implementation of a large-scale direct load control system-some critical factors. IEEE Transactions on Power Apparatus and Systems, PAS-104(7):16631669, July 1985.

[66] J. M. McIntyre, L. Ciecior, A. Kaspar, and D. Castrop. Distributed intelligence in load control: Results of an experiment using demand limiting devices for residential load control. IEEE Transactions on Power Apparatus and Systems, PAS-104(5):11401146, May 1985.

[67] R. F. Bischke and R. A. Sella. Design and controlled use of water heater load management. IEEE Transactions on Power Apparatus and Systems, PAS-104(6):12901293, June 1985.

[68] B. Rautenbach and I. E. Lane. The multi-objective controller: a novel approach to domestic hot water load control. IEEE Transactions on Power Systems, 11(4):18321837, Nov 1996.

[69] C. Ninagawa, K. Taga, A. Kiyota, and T. Yamaguchi. Emulation system on smart grid fast automated demand response of widely-distributed stochastically-operating building facilities. In 2015 IEEE International Symposium on Systems Engineering (ISSE), pages 66-70, Sept 2015. 
[70] W. Mai and C. Y. Chung. Model predictive control based on thermal dynamic building model in the demand-side management. In 2016 IEEE Power and Energy Society General Meeting (PESGM), pages 1-5, July 2016.

[71] M. Motalleb, M. Thornton, E. Reihani, and R. Ghorbani. A nascent market for contingency reserve services using demand response. Applied Energy, 179:985 - 995, 2016.

[72] K. Dehghanpour and S. Afsharnia. Electrical demand side contribution to frequency control in power systems: a review on technical aspects. Renewable and Sustainable Energy Reviews, 41:1267 - 1276, 2015.

[73] F. Rahimi and A. Ipakchi. Demand response as a market resource under the smart grid paradigm. IEEE Transactions on Smart Grid, 1(1):82-88, June 2010.

[74] E. Nekouei, T. Alpcan, and D. Chattopadhyay. Game-theoretic frameworks for demand response in electricity markets. IEEE Transactions on Smart Grid, 6(2):748758, March 2015.

[75] M. Parvania, M. Fotuhi-Firuzabad, and M. Shahidehpour. Optimal demand response aggregation in wholesale electricity markets. IEEE Transactions on Smart Grid, 4(4):1957-1965, Dec 2013.

[76] D. T. Nguyen, M. Negnevitsky, and M. de Groot. Market-based demand response scheduling in a deregulated environment. IEEE Transactions on Smart Grid, 4(4):1948-1956, Dec 2013. 
[77] M. Ali, A. Alahäivälä, F. Malik, M. Humayun, A. Safdarian, and M. Lehtonen. A market-oriented hierarchical framework for residential demand response. International Journal of Electrical Power \& Energy Systems, 69:257 - 263, 2015.

[78] S. P. Burger and M. Luke. Business models for distributed energy resources: A review and empirical analysis. Energy Policy, 109:230 - 248, 2017.

[79] S. Zhang. Innovative business models and financing mechanisms for distributed solar PV (DSPV) deployment in China. Energy Policy, 95:458 - 467, 2016.

[80] E. Funkhouser, G. Blackburn, C. Magee, and V. Rai. Business model innovations for deploying distributed generation: The emerging landscape of community solar in the U.S. Energy Research and Social Science, 10:90 - 101, 2015.

[81] E. Koliou, C. Eid, J. P. Chaves-Ávila, and R. A. Hakvoort. Demand response in liberalized electricity markets: Analysis of aggregated load participation in the German balancing mechanism. Energy, 71:245 - 254, 2014.

[82] Natalie Pruggler. Economic potential of demand response at household level - Are Central-European market conditions sufficient? Energy Policy, 60:487 - 498, 2013.

[83] C.F. Calvillo, A. Sánchez-Miralles, J. Villar, and F. Martin. Optimal planning and operation of aggregated distributed energy resources with market participation. Applied Energy, 182:340 - 357, 2016. 
[84] C. Eid, P. Codani, Y. Perez, J. Reneses, and R. Hakvoort. Managing electric flexibility from distributed energy resources: A review of incentives for market design. Renewable and Sustainable Energy Reviews, 64:237 - 247, 2016.

[85] E. Niesten and F. Alkemade. How is value created and captured in smart grids? A review of the literature and an analysis of pilot projects. Renewable and Sustainable Energy Reviews, 53:629 - 638, 2016.

[86] M. Motalleb, M. Thornton, E. Reihani, and R. Ghorbani. Providing frequency regulation reserve services using demand response scheduling. Energy Conversion and Management, 124:439 - 452, 2016.

[87] A. Roos, S. Ottesen, and T. F. Bolkesjo. Modeling consumer flexibility of an aggregator participating in the wholesale power market and the regulation capacity market. Energy Procedia, 58:79 - 86, 2014. Renewable Energy Research Conference, RERC 2014.

[88] S. Kiliccote, M. A. Piette, E. Koch, and D. Hennage. Utilizing automated demand response in commercial buildings as non-spinning reserve product for ancillary services markets. In 2011 50th IEEE Conference on Decision and Control and European Control Conference, pages 4354-4360, Dec 2011.

[89] S. Rahnama, S. E. Shafiei, J. Stoustrup, H. Rasmussen, and J. Bendtsen. Evaluation of aggregators for integration of large-scale consumers in smart grid. IFAC Proceedings Volumes, 47(3): 1879 - 1885, 2014. 19th IFAC World Congress. 
[90] N. Ruiz, I. Cobelo, and J. Oyarzabal. A direct load control model for virtual power plant management. IEEE Transactions on Power Systems, 24(2):959-966, May 2009.

[91] C. Diduch, M. Shaad, R. Errouissi, M. E. Kaye, J. Meng, and L. Chang. Aggregated domestic electric water heater control - building on smart grid infrastructure. In Proceedings of The 7th International Power Electronics and Motion Control Conference, volume 1, pages 128-135, June 2012.

[92] J. Kondoh, N. Lu, and D. J. Hammerstrom. An evaluation of the water heater load potential for providing regulation service. IEEE Transactions on Power Systems, 26(3):1309-1316, Aug 2011.

[93] T. M. Keep, F. E. Sifuentes, D. M. Auslander, and D. S. Callaway. Using load switches to control aggregated electricity demand for load following and regulation. In 2011 IEEE Power and Energy Society General Meeting, pages 1-7, July 2011.

[94] J.C. Laurent and R.P. Malhame. A physcially-based computer model of aggregate electric water heating loads. IEEE Transactions on Power Systems, 9:1209-1217, Aug 1994.

[95] J.C. Laurent, G Desaulniers, R.P. Malhame, and F. Soumis. A column generation method for optimal load management via control of electric water heaters. IEEE Transactions on Power Systems, 10:1389-1400, Aug 1995.

[96] N. Fitzgerald, A. M. Foley, and E. McKeogh. Integrating wind power using intelligent electric water heating. Energy, 48:135-143, 2012. 
[97] P. Kepplinger, G. Huber, and J. Petrasch. Autonomous optimal control for demand side management with resistive domestic hot water heaters using linerar optimization. Energy and Buildings, 100:50-55, 2015.

[98] Vassilis Kapsalis and Loukas Hadellis. Optimal operation scheduling of electric water heaters underdynamic pricing. Sustainable Cities and Societies, 31:109-121, 2017.

[99] Vassilis Kapsalis, Georgia Safouri, and Loukas Hadellis. Cost/comfort-oriented optimization algorithm for operation scheduling of electric water heaters under dynamic pricing. Journal of Cleaner Production, 198:1053-1065, 2018.

[100] M. Roux, M. Apperley, and M.J. Booysen. Comfort, peak load, and energy: Centralized control of water heaters for demand-driven prioritization. Energy for Sustainable Development, 44:78-86, 2018.

[101] Jianing Li, Zhi Wu, Suyang Zhou, Hao Fu, and Xiao-Ping Zhang. Aggretator service for PV and battery energy storage systems of residential building. CSEE Journal of POWER and Energy Systems, 44:78-86, 2018.

[102] Tasnimun Faika, Taesic Kim, and Maleq Khan. An Internet of Things (IoT)-based network for dispersed and decentralized wireless battery management systems. 2018 IEEE Transportation Electrification Conference and Expo, pages 1060-1064, June 2018. 
[103] M. Khalid and A.V. Savkin. Minimization and control of battery energy storage for wind power smoothing: Aggregated, distributed, and semi-distributed storage. Renewable Energy, 44:78-86, 2018.

[104] Georgia E. Asimakopoulou and Nikos D. Hatziargyriou. Evaluation of economic benefits of DER aggregation. Energy, 9:499-510, Apr 2018.

[105] C.F. Calvillo, A. Sancherz-Miralles, J. Villar, and F. Martin. Optimal planning and operation of aggregated distributed energy resources with market participation. Applied Energy, 182:340-357, 2016.

[106] Marina Gonzalez Vaya and Goran Andersson. Electric vehicle aggregator/system operator coordination for charging scheduling and services procurement. IEEE Transactions on Power Systems, 30(5):2375-2385, September 2015.

[107] Stylianos I. Vagropoulos and Anastasios G. Bakirtzis. Optimal bidding strategy for electric vehicle aggregators in electricity markets. IEEE Transactions on Power Systems, 28(4):4031-4041, November 2013.

[108] M. R. Sarker, Y. Dvorkin, and M. A. Ortega-Vazquez. Optimal participation of an electric vehicle aggregator in day-ahead energy and reserve markets. IEEE Transactions on Power Systems, 31(5):3506-3515, September 2016.

[109] A. Maleki, M. G. Khajeh, and M. Ameri. Optimal sizing of a grid independent hybrid renewable energy system incorporating resource uncertainty, and load uncertainty. International Journal of Electrical Power \& Energy Systems, 83:514 - 524, 2016. 
[110] T. Zhou and W. Sun. Optimization of battery-super capacitor hybrid energy storage station in wind/solar generation system. IEEE Transactions on Sustainable Energy, 5(2):408-415, April 2014.

[111] N. H. Saad, A. A. El-Sattar, and A. M. Mansour. Improved particle swarm optimization for photovoltaic system connected to the grid with low voltage ride through capability. Renewable Energy, 85:181 - 194, 2016.

[112] P. Kayal and C.K. Chanda. Optimal mix of solar and wind distributed generations considering performance improvement of electrical distribution network. Renewable Energy, 75:173 - 186, 2015.

[113] T. P. Chang. Wind energy assessment incorporating particle swarm optimization method. Energy Conversion and Management, 52(3):1630 - 1637, 2011.

[114] G.R. Aghajani, H.A. Shayanfar, and H. Shayeghi. Presenting a multi-objective generation scheduling model for pricing demand response rate in micro-grid energy management. Energy Conversion and Management, 106:308 - 321, 2015.

[115] H. Shahinzadeh, S. H. Fathi, M. Moazzami, and S. H. Hosseinian. Hybrid big bangbig crunch algorithm for solving non-convex economic load dispatch problems. In 2017 2nd Conference on Swarm Intelligence and Evolutionary Computation (CSIEC), pages 48-53, March 2017.

[116] G. F. Reid and L. Hasdorff. Economic dispatch using quadratic programming. IEEE Transactions on Power Apparatus and Systems, PAS-92(6):2015-2023, Nov 1973. 
[117] N. Sinha and B. Purkayastha. PSO embedded evolutionary programming technique for nonconvex economic load dispatch. In IEEE PES Power Systems Conference and Exposition, 2004., pages 66-71 vol.1, Oct 2004.

[118] S. Ahmadi and S. Abdi. Application of the Hybrid Big Bang - Big Crunch algorithm for optimal sizing of a stand-alone hybrid PV/wind/battery system. Solar Energy, $134: 366-374,2016$.

[119] G. R. Newsham, B. J. Birt, and I.H. Rowlands. A comparison of four methods to evaluate the effect of a utility residential air-conditioner load control program on peak electricity use. Energy Policy, 39(10):6376 - 6389, 2011. Sustainability of biofuels.

[120] S. Aslam, Z. Iqbal, N. Javaid, Z. Ali-Khan, K. Aurangzeb, and S. Itaza-Haide. Towards efficient energy management of smart buildings exploiting heuristic optimization with real time and critical peak pricing schemes. Energies, 10:2065, 2017.

[121] K. Al-jabery, Z. Xu, W. Yu, D. C. Wunsch, J. Xiong, and Y. Shi. Demand-side management of domestic electric water heaters using approximate dynamic programming. IEEE Transactions on Computer-Aided Design of Integrated Circuits and Systems, 36(5):775-788, May 2017.

[122] M.A. Elhadidy and S.M. Shaahid. Parametric study of hybrid (wind + solar + diesel) power generating systems. Renewable Energy, 21(2):129 - 139, 2000. 
[123] H. G. Beyer and C. Langer. A method for the identification of configurations of PV/wind hybrid systems for the reliable supply of small loads. Solar Energy, 57(5):381 - 391, 1996.

[124] J. L. Bernal-Agustín and R. Dufo-López. Simulation and optimization of standalone hybrid renewable energy systems. Renewable and Sustainable Energy Reviews, 13(8):2111 - 2118, 2009.

[125] D. Brunelli and L. Tamburini. Residential load scheduling for energy cost minimization. In 2014 IEEE International Energy Conference (ENERGYCON), pages 675-682, May 2014.

[126] F. Mangiatordi, E. Pallotti, P. Del Vecchio, and F. Leccese. Power consumption scheduling for residential buildings. In 2012 11th International Conference on Environment and Electrical Engineering, pages 926-930, May 2012.

[127] G. Kardaras, A. Rosselló-Busquet, V. B. Iversen, J. Soler, and L. Dittmann. Regulating electricity demand peaks for home appliances using reversible fair scheduling. In 2010 IEEE 4th International Conference on Internet Multimedia Services Architecture and Application, pages 1-6, Dec 2010.

[128] O. Babacana, E. Ratnam, V. Disfania, and J. Kleissla. Distributed energy storage system scheduling considering tariff structure, energy arbitrage and solar PV penetration. Applied Energy, 205:1384-1393, 2017. 
[129] B.Passenberg, M.Leibold, O.Stursberg, and M. Buss. A globally convergent, locally optimal min-h algorithm for hybrid optimal control. In IAM Journal on Control and Optimization, volume 52, pages 718-746, 2014.

[130] E. L. Barrett. The Investigation and Optimization of a Two-Heat-Pump System Incorporating Thermal Storage for Shaping Residential Heating Load. Portland State University, 2016. Masters thesis.

[131] R. B.Rector. Generalized Differential Calculus and Applications to Optimization. Portland State University, 2017. Doctoral Dissetation.

[132] S. H. Lee and C. L. Wilkins. A practical approach to appliance load control analysis: A water heater case study. IEEE Transactions on Power Apparatus and Systems, PAS-102(4):1007-1013, April 1983.

[133] O. Ma, N. Alkadi, P. Cappers, P. Denholm, J. Dudley, S. Goli, M. Hummon, S. Kiliccote, J. MacDonald, N. Matson, D. Olsen, C. Rose, M. D. Sohn, M. Starke, B. Kirby, and M. O'Malley. Demand response for ancillary services. IEEE Transactions on Smart Grid, 4(4):1988-1995, Dec 2013.

[134] O. Siddiqui. Assessment of achievable potential from energy efficiency and demand response programs in the u.s. (2010-2030). Electric Power Research Institute, Tech. Rep. 1018363, January 2009. 
[135] S. S. George J. Bode P. Mangasarian I. Rohmund G. Wikler D. Ghosh A. Faruqui, R. Hledik and S. Yoshida. A national assessment of demand response potential. Federal Energy Regulatory Commission, Tech. Rep., June 2009.

[136] B. Helms M. Starke B. Kirby D. Todd, M. Caulfield and J. Kueck. Providing reliability services through DR: A preliminary evaluation of the DR capabilities of Alcoa Inc. Oak Ridge National Laboratory, Tech. Rep. ORNL/TM 2008/233, January 2009.

[137] C. Goldman P. Cappers and D. Kathan. Demand response in U.S. electricity markets, empirical evidence. Lawrence Berkeley National Laboratory, Tech. Rep. LBNL-2124E, June 2009.

[138] B. Kirby. Spinning reserve from responsive loads. Oak Ridge National Laboratory, Tech. Rep. ORNL/TM 2003/19, March 2003.

[139] G. Mahalakshmi, S. Sridevi, and S. Rajaram. A survey on forecasting of time series data. In 2016 International Conference on Computing Technologies and Intelligent Data Engineering (ICCTIDE'16), pages 1-8, Jan 2016.

[140] K. Greff, R. K. Srivastava, J. Koutník, B. R. Steunebrink, and J. Schmidhuber. Lstm: A search space odyssey. IEEE Transactions on Neural Networks and Learning Systems, 28(10):2222-2232, Oct 2017.

[141] T. Panapongpakorn and D. Banjerdpongchai. Short-term load forecast for energy management systems using time series analysis and neural network method with 
average true range. In 2019 First International Symposium on Instrumentation, Control, Artificial Intelligence, and Robotics (ICA-SYMP), pages 86-89, Jan 2019.

[142] V. De, T. T. Teo, W. L. Woo, and T. Logenthiran. Photovoltaic power forecasting using lstm on limited dataset. In 2018 IEEE Innovative Smart Grid Technologies Asia (ISGT Asia), pages 710-715, May 2018.

[143] Junyoung Chung, Caglar Gulcehre, Kyunghyun Cho, and Yoshua Bengio. Empirical evaluation of gated recurrent neural networks on sequence modeling. In NIPS 2014 Workshop on Deep Learning, December, 2014.

[144] Jiandong Wang and Wei Xu. Chapter 8 - present situation and problems of large-scale wind power transmission and accommodation policy. In Ningbo Wang, Chongqing Kang, and Dongming Ren, editors, Large-Scale Wind Power Grid Integration, pages 257 - 276. Academic Press, Oxford, 2016.

[145] Robert Hendron. DHW event schedule generator. U.S. Department of Energy, March 2011.

[146] U.S. Beureau of the Census. U.S. residential household data. American FactFinder, 2018.

[147] C. Opathella, A. Elkasrawy, A. A. Mohamed, and B. Venkatesh. Optimal scheduling of merchant-owned energy storage systems with multiple ancillary services. IEEE Open Access Journal of Power and Energy, 7:31-40, Dec 2020. 
[148] FERC. Performance-based regulation: Maximizing value through markets. In Federal Energy Regulatory Commission, volume Order 755, October 2011.

[149] R. Ogburn and S. Bresler. Performance-based regulation: Maximizing value through markets. In 2012 IEEE Power and Energy Society General Meeting, pages 1-5, July 2012.

[150] J. W. O'Sullivan and M. J. O'Malley. Economic dispatch of a small utility with a frequency based reserve policy. IEEE Transactions on Power Systems, 11(3):16481653, Aug 1996.

[151] E. Ela, V. Gevorgian, A. Tuohy, B. Kirby, M. Milligan, and M. O’Malley. Market designs for the primary frequency response ancillary service: Motivation and design. IEEE Transactions on Power Systems, 29(1):421-431, Jan 2014.

[152] E. Ela, V. Gevorgian, A. Tuohy, B. Kirby, M. Milligan, and M. O’Malley. Market designs for the primary frequency response ancillary service. In 2014 IEEE PES General Meeting | Conference Exposition, pages 1-1, July 2014.

[153] T. Slay. Adoption of an Internet of Things Framework for Distributed Energy Resource Coordination and Control. Portland State University, 2018. Masters thesis.

[154] Prabha Kundur. Power system stability and control. McGraw-Hill, January 1994. 


\section{Appendix A: MATLAB ${ }^{\circledR}$ Implementation of AWHS Optimization, stage 1}

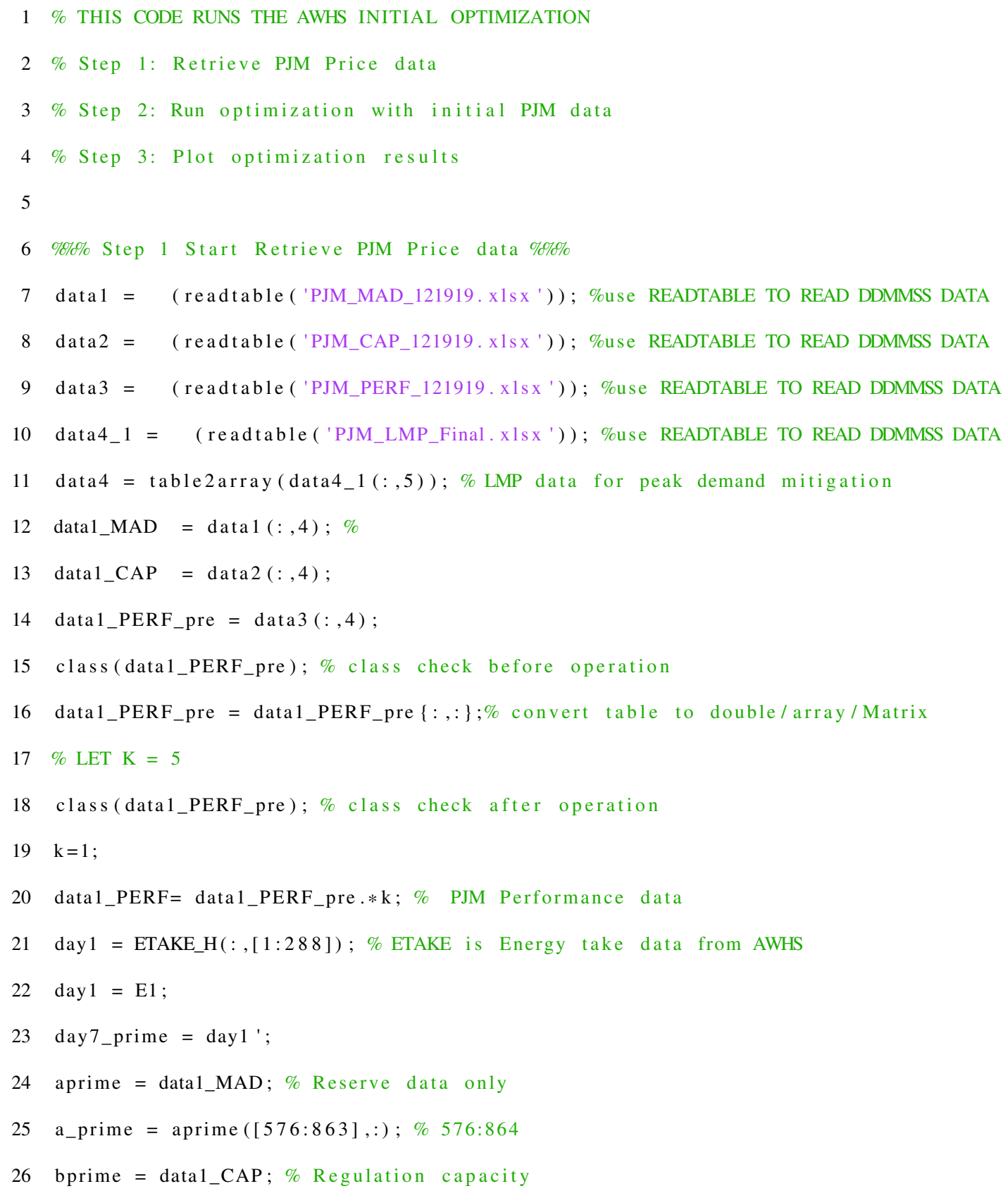




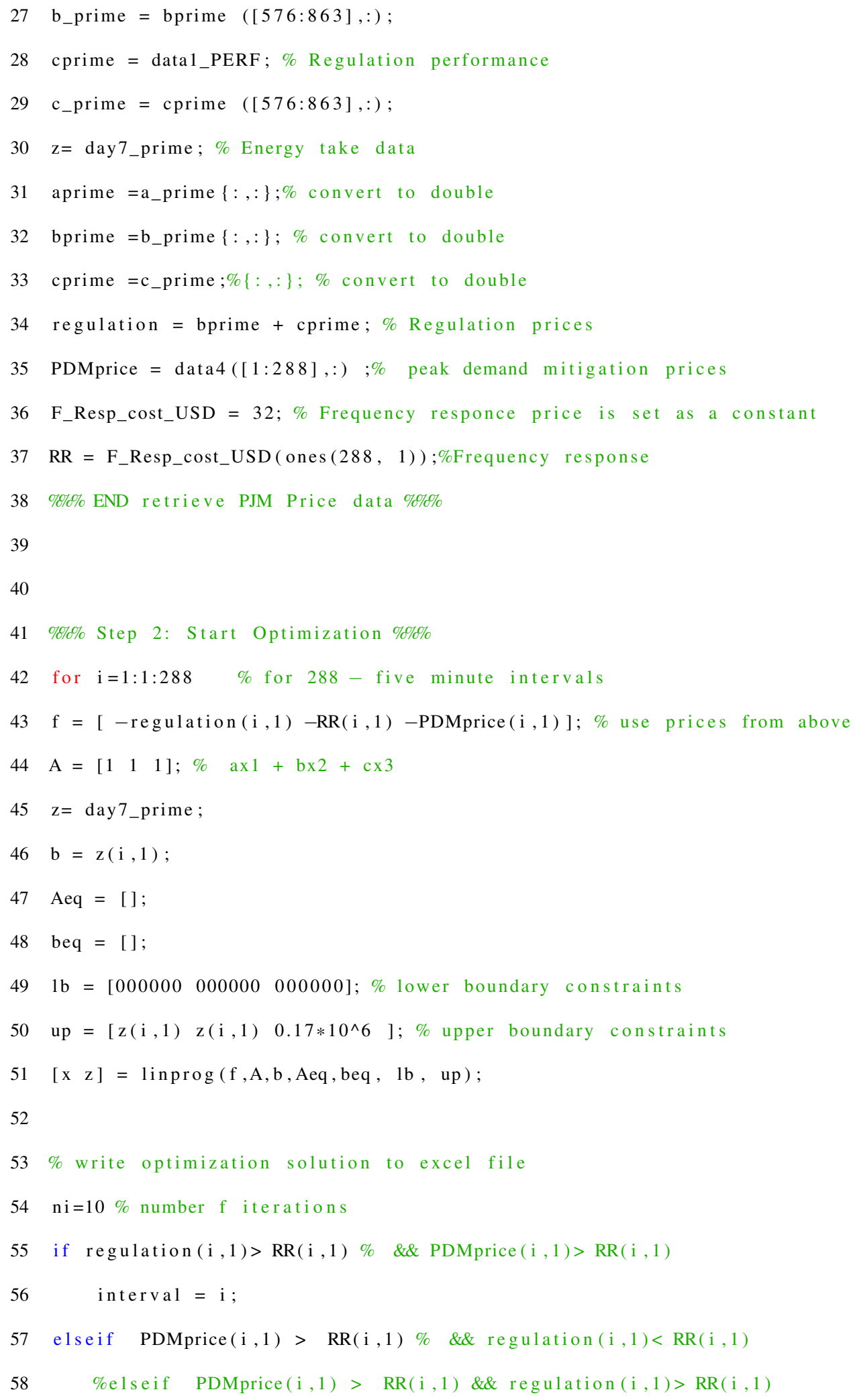




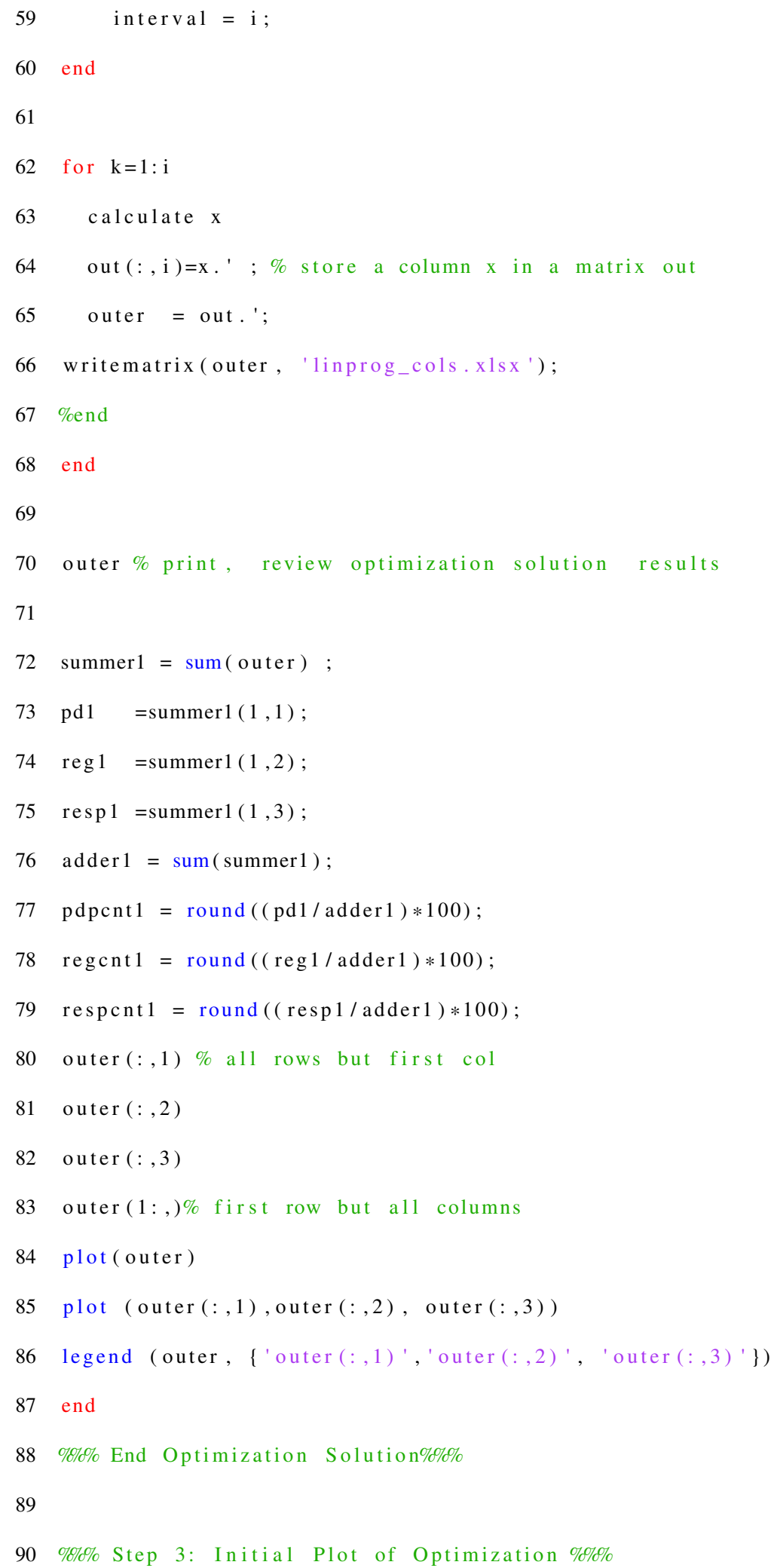




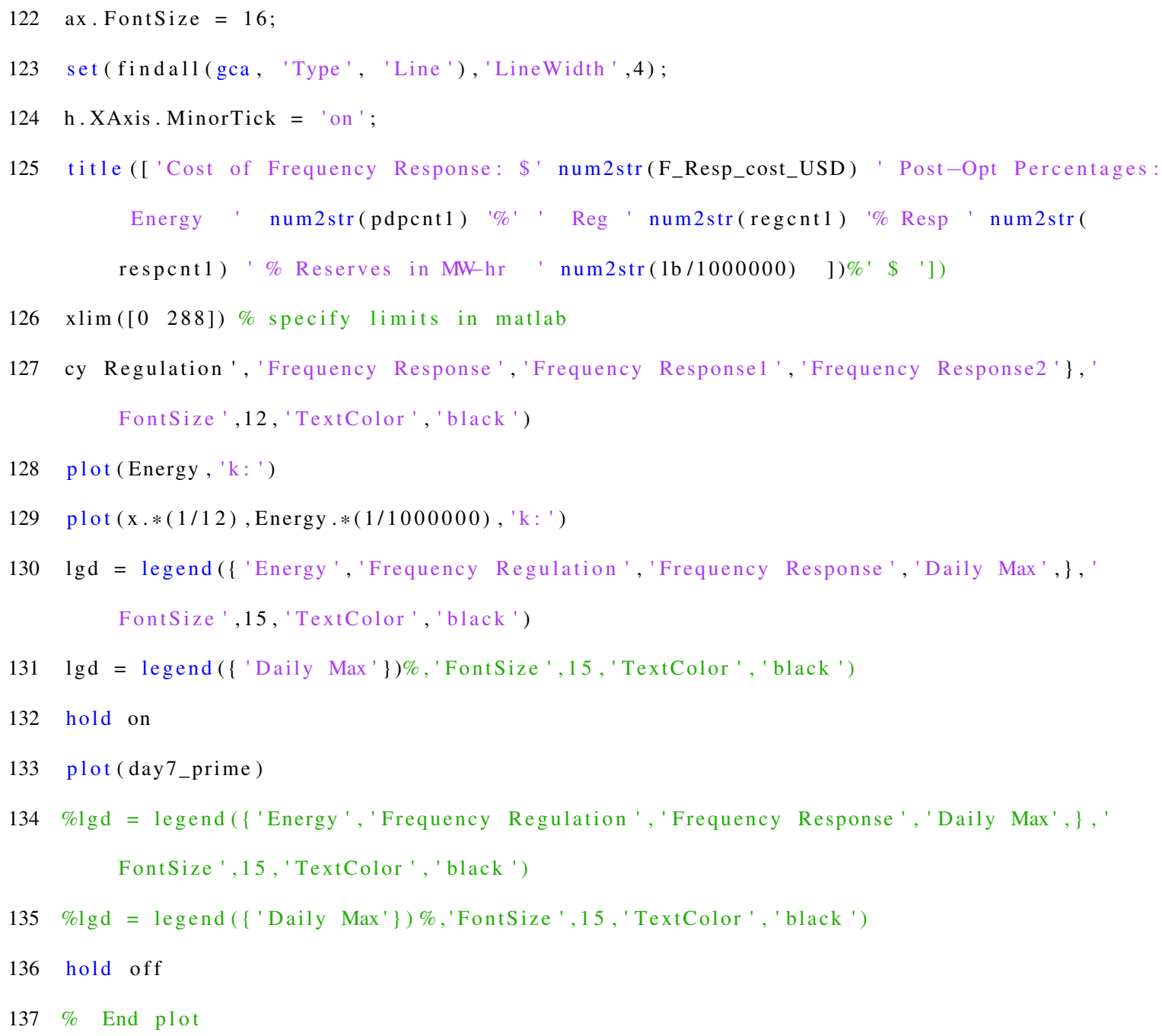




\section{Appendix B: MATLAB ${ }^{\circledR}$ Implementation of Test and Training using LSTM}

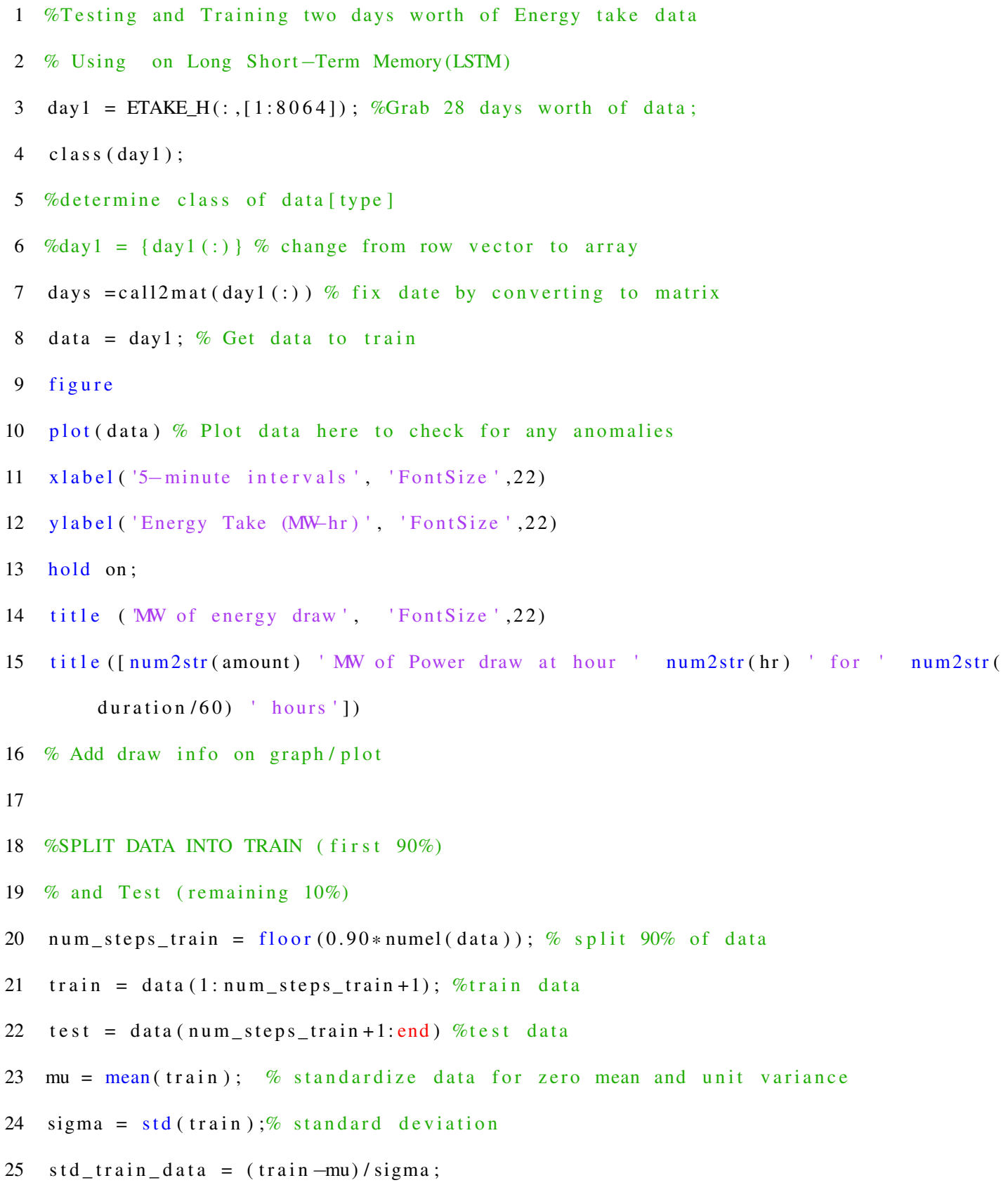




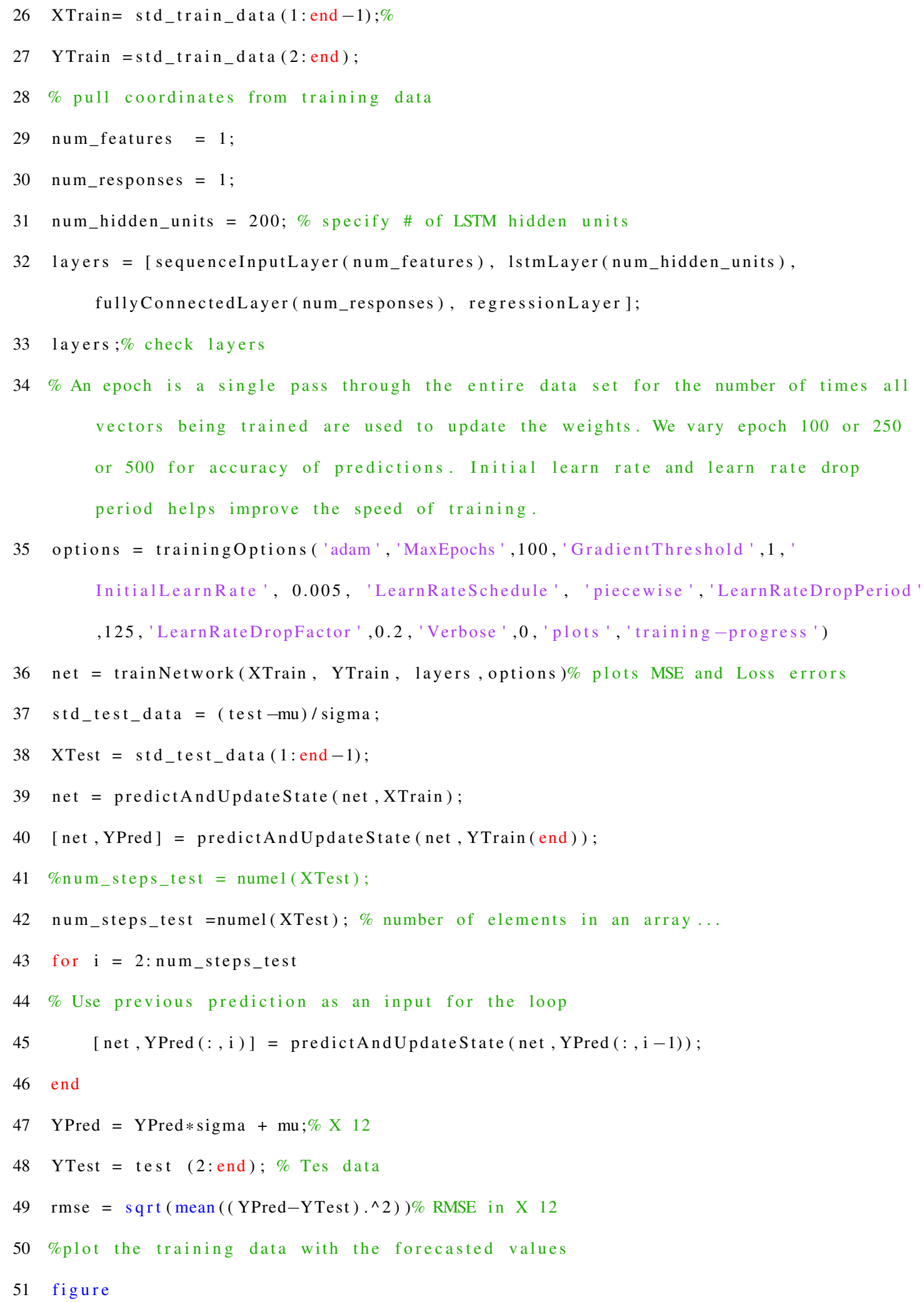




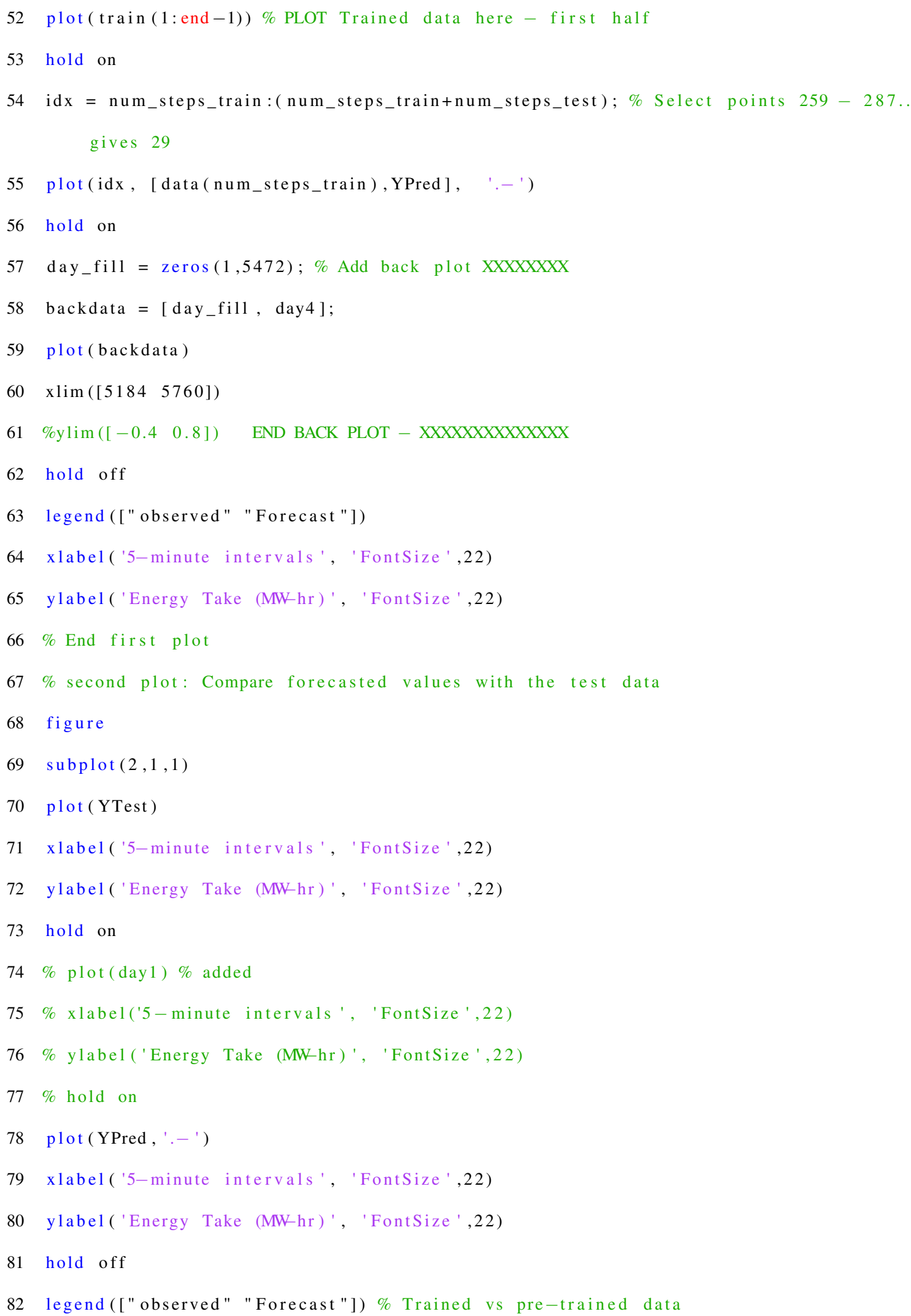




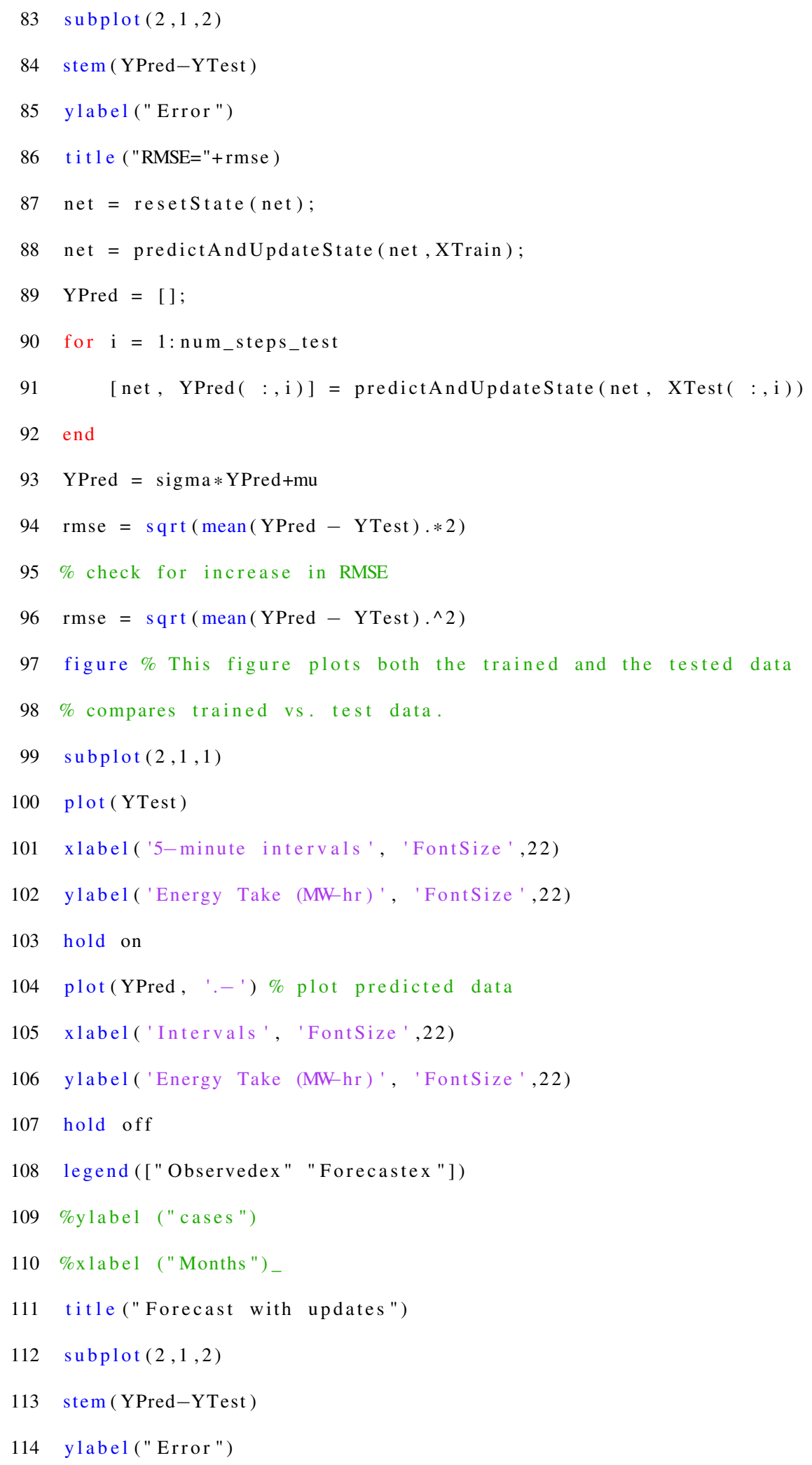


115 xlabel ("5-minute intervals")

116 title ("RMSE $="+$ rmse) \% Plot RMSE results after testing 


\section{Appendix C: MATLAB ${ }^{\circledR}$ Implementation of AWHS Optimization, stage 2}

2

3 function [kmatrix, sellmatrix] = sell(outer)

4 \% Function outer is input, kmatrix and sell matrix is output

$5 \operatorname{ctr}=0$

$6 \quad$ livebit $=0$;

$7 \quad$ iveint $=0$;

8 livectr $=0$;

9 SIZE $=$ size (outer);

10 kmatrix $=$ zeros $(\operatorname{SIZE}(1), 5) ;$

11 for $\mathrm{n}=1: \operatorname{SIZE}(1)$

$12 \operatorname{ctr}=\operatorname{ctr}+1$;

13 kmatrix $(\mathrm{n}, 1)=\operatorname{ctr} ; \%$ Interval between dispatches goes here

14 if $((\operatorname{outer}(\mathrm{n}, 2)>\operatorname{outer}(\mathrm{n}, 3)) \|(\operatorname{louter}(\mathrm{n}, 2)>\operatorname{outer}(\mathrm{n}, 1)) \quad \mathrm{II}($ outer $(\mathrm{n}, 3)>$

outer $(n, 1)) \|$ ( outer $(n, 3)>$ outer $(n, 2))$ ) Regulation only

17 end

18 if $($ livebit $==0 \& \&$ kmatrix $(n, 2)==1)$

$19 \quad$ livebit $=1$;

$20 \quad$ liveint $=n$;

$21 \quad$ livectr $=1$; 


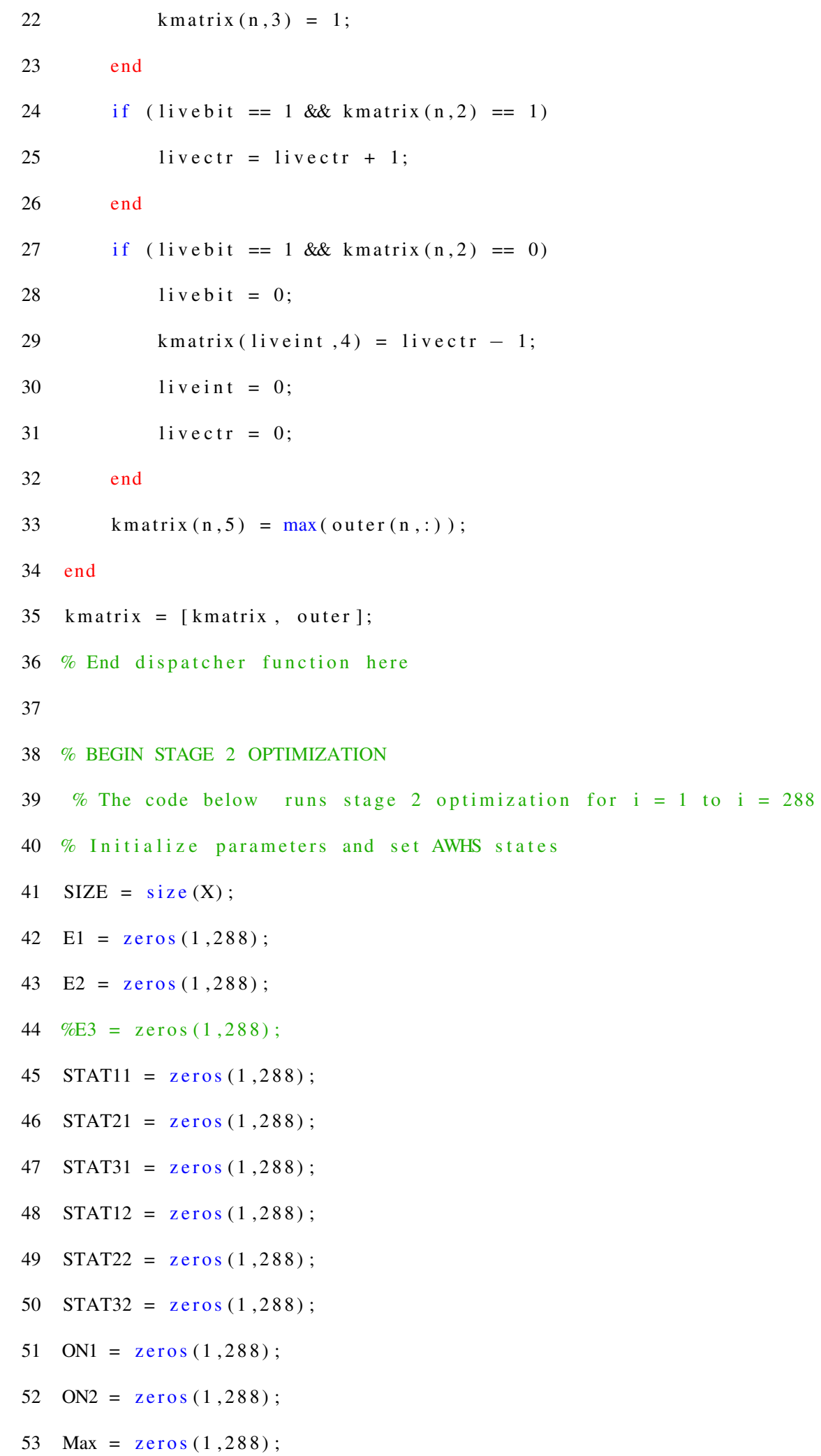




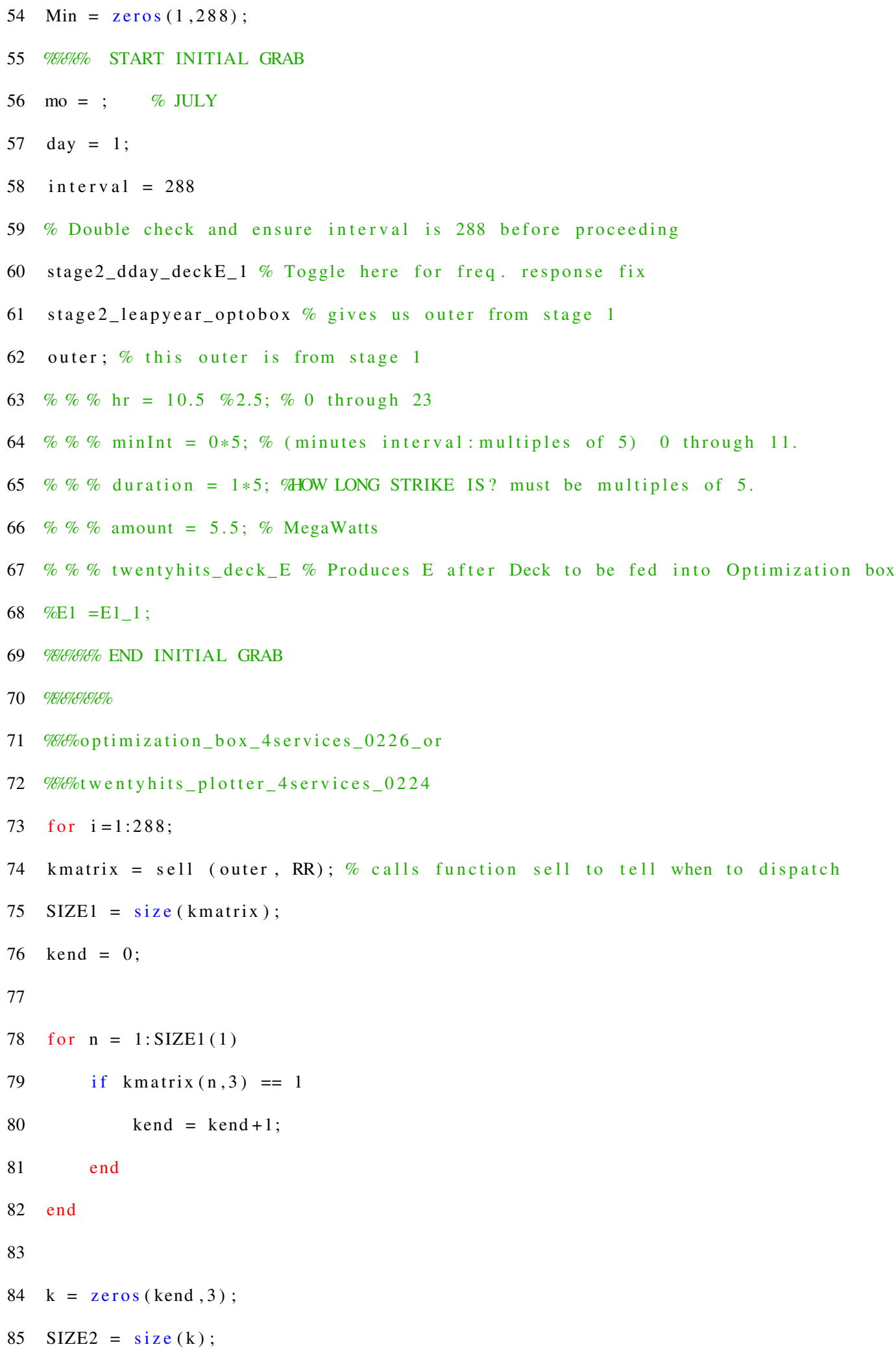




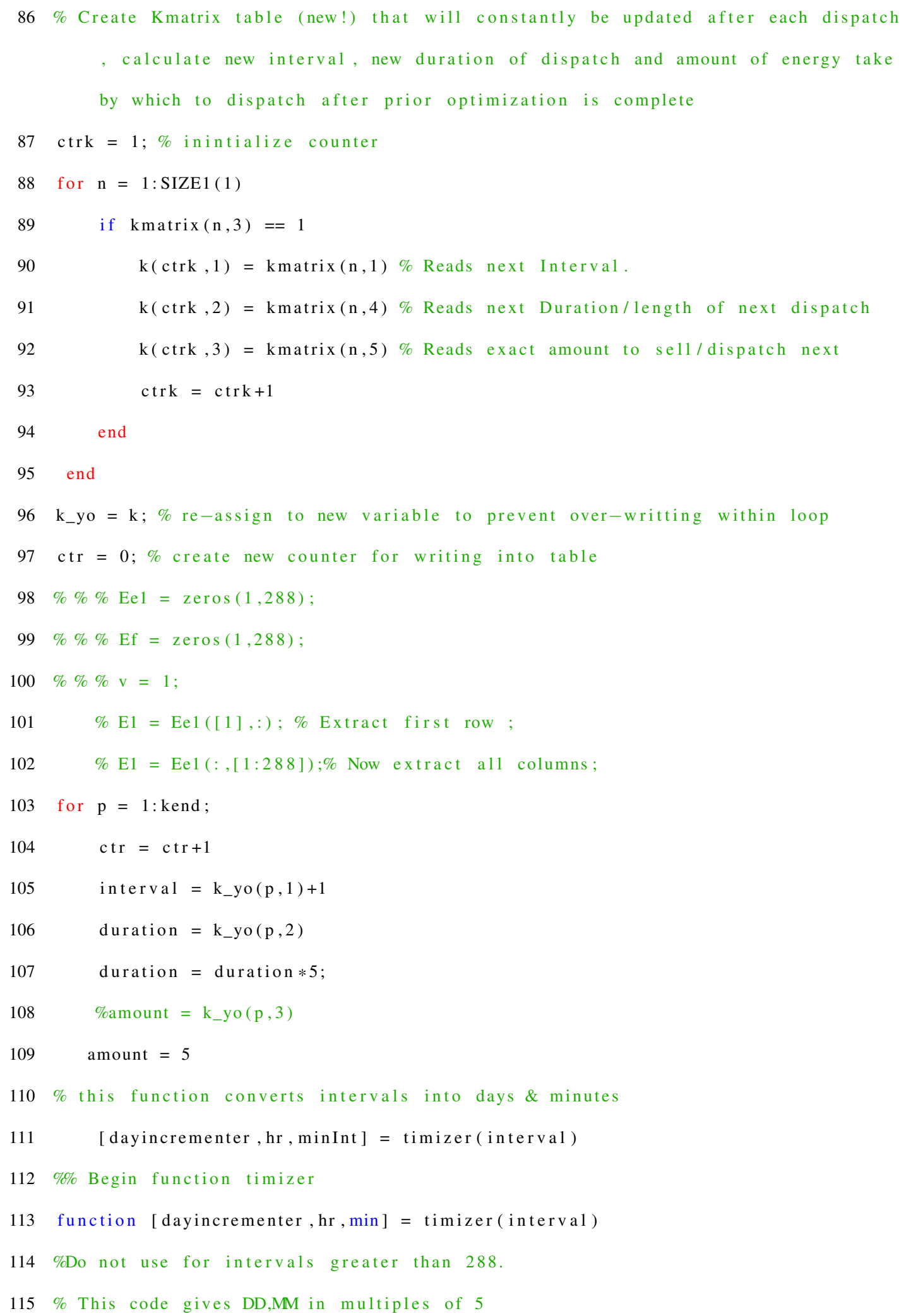




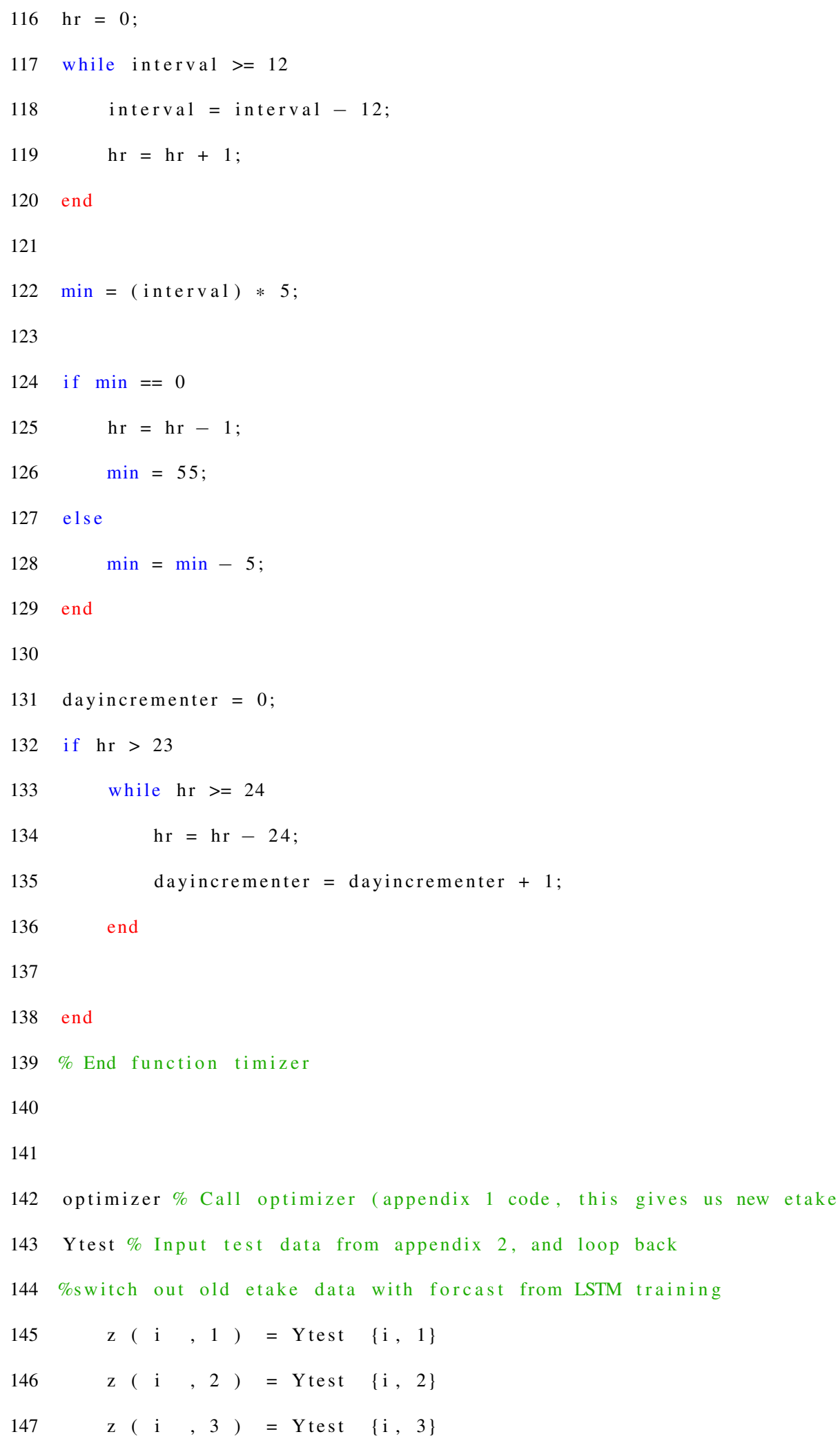




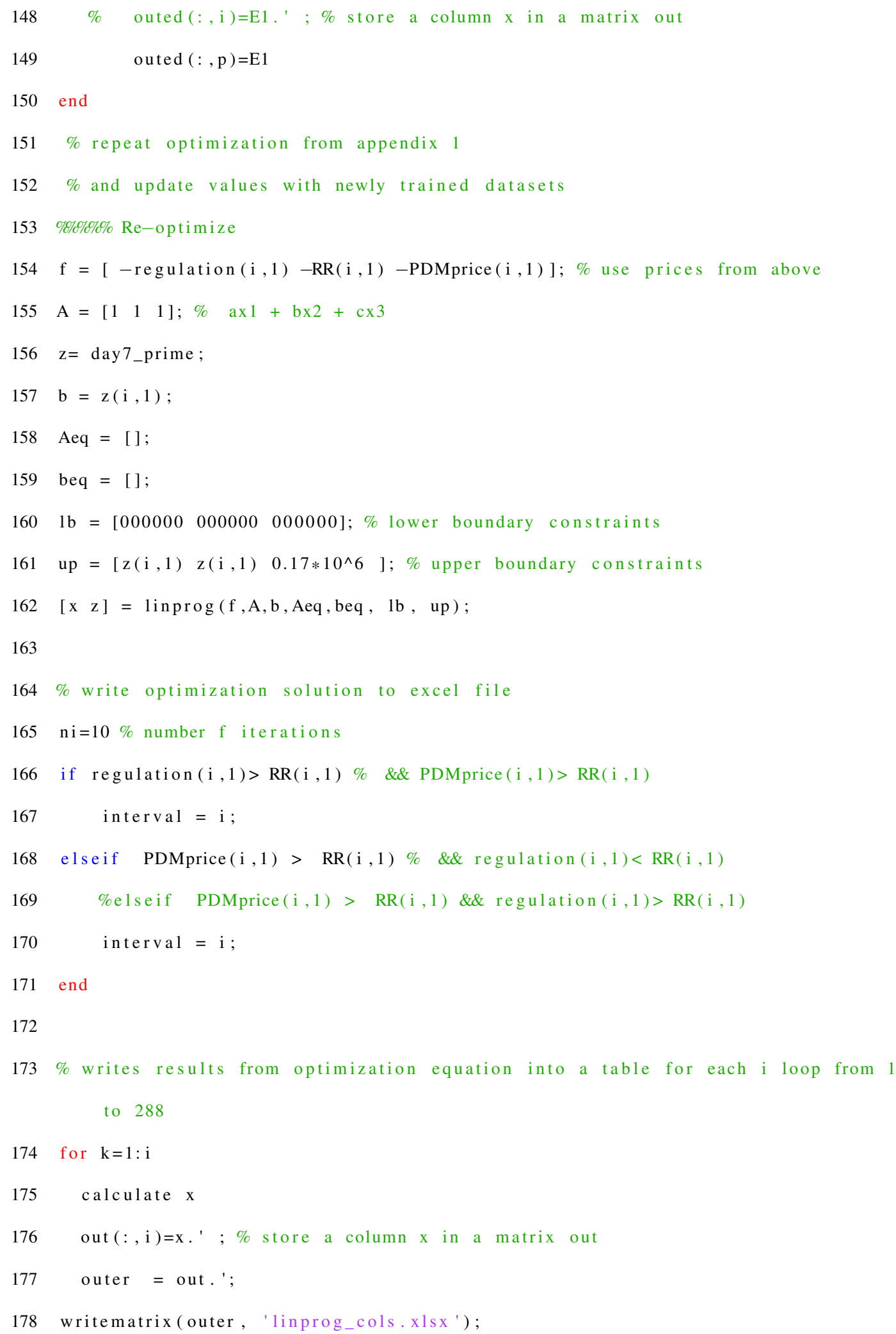


180 end

181

182 outer \% print, review optimization solution results

$183 \% \% \% \%$ End re-optimize

184

$185 \%$ Loop from $\mathrm{i}=1$ to $\mathrm{i}=288$, dispatch, optimize, shift and repeat

186 end 